\title{
Tracking Assembly Kinetics of Intermediate Filaments
}

\author{
Dissertation \\ for the award of the degree \\ "Doctor rerum naturalium" \\ of the Georg-August-Universität Göttingen \\ within the doctoral program \\ Göttingen Graduate School for Neurosciences, \\ Biophysics, and Molecular Biosciences (GGNB) \\ of the Georg-August University \\ School of Science (GAUSS)
}

submitted by

Oliva Saldanha

from Virajpet (India)

Göttingen, 2016 



\section{Thesis Committee:}

Prof. Dr. Sarah Köster

Institute for X-Ray Physics

Georg-August-Universität Göttingen

Prof. Dr. Simone Techert

Institute for X-Ray Physics

Georg-August-Universität Göttingen

Prof. Dr. Jean-Christophe Baret

1. University of Bordeaux, Centre de Recherche Paul Pascal (CNRS)

2. Max Planck Institute for Dynamics and Self-Organization, Göttingen 


\section{Members of the Examination Board:}

Referee: Prof. Dr. Sarah Köster

Institute for X-Ray Physics

Georg-August-Universität Göttingen

$2^{\text {nd }}$ referee: Dr. Jochen Hub

Department of Molecular Structural Biology

Georg-August-Universität Göttingen

\section{Further members of the Examination Board:}

Prof. Dr. Jean-Christophe Baret

1. University of Bordeaux, Centre de Recherche Paul Pascal (CNRS)

2. Max Planck Institute for Dynamics and Self-Organization, Göttingen

Prof. Dr. Simone Techert

Institute for X-Ray Physics

Georg-August-Universität Göttingen

Dr. Thomas Burg

Department for Biological Micro- and Nanotechnology

Max Planck Institute for Biophysical Chemistry, Göttingen

Prof. Dr. Detlev Schild

Department of Neurophysiology \& Cellular Biophysics

Georg-August-Universität Göttingen

\section{Date of Oral Examination:}




\section{Contents}

\begin{tabular}{lll}
\hline 1 & Introduction & 1
\end{tabular}

2 Hierarchical Architecture of Intermediate Filaments 3

2.1 Organization in the Cell $\ldots \ldots \ldots \ldots \ldots$

2.2 Intermediate Filaments . . . . . . . . . . . . . . . . 5

2.3 Vimentin . . . . . . . . . . . . . . . . . . 7

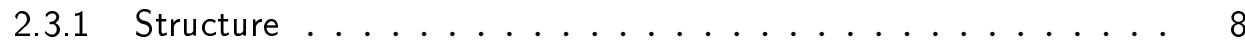

2.4 Purification and Reconstitution of Vimentin . . . . . . . . . . . 10

2.4 .1 Dialysis and Assembly Techniques. . . . . . . . . . . . . . 11

2.4 .2 Estimation of Protein Concentration by UV-Vis Spectroscopy . . 13

2.5 Role of lons in Assembly Process . . . . . . . . . . . . . . . . . 13

3 Microfluidics for in situ Investigation of Bio-Processes 15

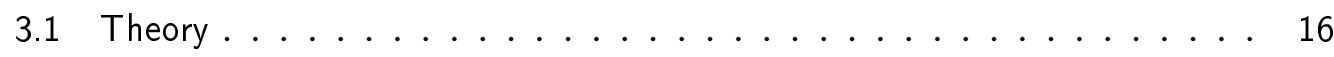

3.1.1 "Life at Low Reynolds Number" [59] . . . . . . . . . . . . . . . . 16

3.1 .2 Diffusion in Microsystems . . . . . . . . . . . . . 18

$3.2 \quad$ Microfluidic Fabrication Techniques . . . . . . . . . . . . . . . . 19

3.2 .1 Photolithography . . . . . . . . . . . . . . . . . . 19

3.2 .2 Soft lithography $\ldots \ldots \ldots \ldots \ldots \ldots$ 
Contents

3.3 Motivation for Device Geometries . . . . . . . . . . . . . . . . 25

3.3 .1 Continuous Flow Microfluidics . . . . . . . . . . . . . . . . 25

3.3 .2 Droplet-Based Microfluidics . . . . . . . . . . . . 26

3.4 Device Implementation in Experiments . . . . . . . . . . . . . . 27

3.4 .1 Continuous-Flow Devices in SAXS Experiments . . . . . . . . . 27

3.4 .2 Droplet-Based Devices in SAXS Experiments . . . . . . . . . 28

$3.5 \quad$ FEM Simulations for Tracing Species-Distribution . . . . . . . . . . . 30

3.5 .1 Model Geometry and Mesh . . . . . . . . . . . . 32

3.5.2 Global Constants and Varying Parameters . . . . . . . . . . . 33

3.5 .3 Representative Results from Simulations . . . . . . . . . . . . 33

4 Small Angle X-ray Scattering for Protein Assembly Kinetics 39

4.1 General Theory . . . . . . . . . . . . . . . . . . . . . . . 40

4.1 .1 Form Factor and Structure Factor . . . . . . . . . . . . . . 42

4.1 .2 Concept of Contrast in Particle Solutions. . . . . . . . . . . . 44

4.2 Methodology of Data Treatment . . . . . . . . . . . . . . . . 45

4.2 .1 Resolution Limit . . . . . . . . . . . . . . . . . . . . . . 45

4.2 .2 Guinier Approximation . . . . . . . . . . . . . . . . . . . 46

4.2 .3 Porod's Law . . . . . . . . . . . . . . . . . . . . 48

4.3 Experimental Setups at Synchrotrons . . . . . . . . . . . . . . . . 51

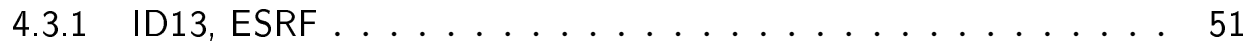

4.3 .2 AustroSAXS, ELETTRA Sincrotrone Trieste . . . . . . . . . . 53

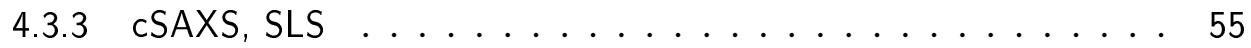

4.3 .4 Comparison of the Different Setups . . . . . . . . . . . . . 55 
4.4 Continuous-Flow Experiments . . . . . . . . . . . . . . . 56

4.4 .1 Results from Experiments . . . . . . . . . . . . . . . . 56

4.4 .2 Data Interpretation and Discussion . . . . . . . . . . . 61

4.5 Droplet Experiments . . . . . . . . . . . . . . . . . 6 65

4.5 .1 Results from Measurements at ID13, ESRF . . . . . . . . . 66

4.5 .2 Results from Measurements at AustroSAXS, ELETTRA . . . . . 70

4.5 .3 Results from Measurements at cSAXS, SLS . . . . . . . . . . 71

$4.5 .4 \quad$ Data Interpretation and Discussion . . . . . . . . . . . . 74

4.6 Summary and Closing Remarks . . . . . . . . . . . . . . . . . . . . 79

5 Time-resolved Light Scattering for Vimentin Kinetics 83

5.1 Theory . . . . . . . . . . . . . . . . . . 85

5.1 .1 Static Light Scattering . . . . . . . . . . . . . . . . . . 85

5.1 .2 Dynamic Light Scattering . . . . . . . . . . . . . . . . 88

5.2 Experimental Section . . . . . . . . . . . . . . . . . . . . 91

5.2 .1 Determination of Protein Concentration . . . . . . . . . . . 91

5.2 .2 Preparation for Light Scattering Experiments . . . . . . . . . . . 91

5.2 .3 Combined Setup for Time-Resolved SLS and DLS . . . . . . . . . 91

5.2 .4 Treatment of Scattering Data . . . . . . . . . . . . . . . 93

5.3 Results from Combined SLS and DLS Experiments . . . . . . . . . . . 93

5.3 .1 Influence of KCL on Kinetics of Vimentin . . . . . . . . 93

5.3 .2 Two Step Kinetic Model . . . . . . . . . . . . . . . . . . . . . . 96

5.3.3 Results for Variabiltiy in Vimentin Concentration . . . . . . . . 98

5.4 Summary and Closing Remarks . . . . . . . . . . . . . . . 100 
Contents

6 Summary and Conclusion 101

6.1 Open Questions and Outlook . . . . . . . . . . . . . . . . . 105

6.2 Structural Quantification of Vimentin . . . . . . . . . . . . 106

\begin{tabular}{|lr}
\hline A Purification of Vimentin & 109
\end{tabular}

B Matlab Code for Evaluating Time Evolution from COMSOL 5.0 113

\begin{tabular}{lll}
\hline C Results from FEM Simulations & 123
\end{tabular}

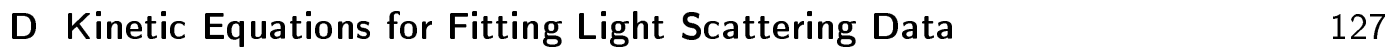

\begin{tabular}{ll}
\hline Bibliography & 135
\end{tabular}

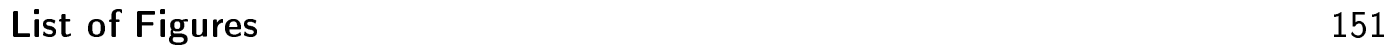

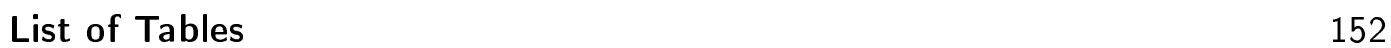

\begin{tabular}{|lr}
\hline List of Abbreviations & 153
\end{tabular}

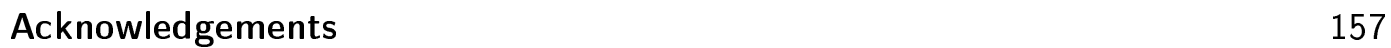

\begin{tabular}{ll}
\hline List of publications & 161
\end{tabular}

\begin{tabular}{ll}
\hline Curriculum Vitae & 163
\end{tabular} 


\section{1 \\ Introduction}

Life exhibits varying degrees of organization, all the way from atoms to molecules to organelles to cells and so on. The structural organization of metazoan cells and their shape are established through the coordinated interaction of a composite network consisting of three individual filament systems, collectively termed the cytoskeleton. Specifically, microtubules and actin filaments, which assemble from monomeric globular proteins, provide polar structures that serve motor proteins as tracks. In contrast, intermediate filaments (IFs) assemble from highly charged, extended coiled-coils in a hierarchical assembly mechanism of lateral and longitudinal interaction steps into non-polar structures. IF proteins are expressed in a distinctly tissue-specific way and thereby serve to generate the precise flexibility of the respective cells and tissues. Accordingly, in the cell, numerous parameters such as $\mathrm{pH}$, salt concentration, ion interaction and osmotic pressure are adjusted such that the generation of functional networks is ensured.

In this thesis, we transfer the problem of the mesenchymal IF protein vimentin to an in vitro setting by combining simulation methods and a variety of experimental techniques such as microfluidics, small angle X-ray scattering (SAXS) and light scattering (LS), where the structural information of vimentin is obtained based on varying ionic constitution. 


\section{\begin{tabular}{l|l} 
Chapter 1 & INTRODUCTION
\end{tabular}}

The structure of this thesis is such that each experimental method used in this work has a dedicated chapter which encompasses the individual theory, state-ofthe art, experimental setup together with the results and discussion obtained in relation to vimentin. A small prelude of each chapter is given below:

In Chapter 2, significance of vimentin in the biological setting is introduced. Purification and reconstitution of vimentin is discussed which is based on the recombinant scheme of protein synthesis. Towards the end of the chapter, insights and ideas in which our studies are steered are briefly discussed.

In Chapter 3, we demonstrate the microfluidic techniques involved in the domain of this thesis. Two main flow techniques are addressed: the continuous flow and droplet flow. Fabrication processes are discussed and potential advantages and disadvantages of the different flow techniques are shown in this chapter.

In Chapter 4, intricacies of SAXS are dealt with regard to protein systems. We study the assembly and aggregation process is two different microfluidic settings: the continuous flow one-phase microfluidics and droplet-based two phase microfluidics. Continuous flow data are discussed in the regime of elucidating surface properties of assembling vimentin intermediates, while the droplet data are a demonstration of state-of-the-art combination of SAXS and a high resolution measurement technique.

In Chapter 5, light scattering is employed as a bridging technique which renders length scales inaccessible by other techniques to study the elongation process of vimentin. Modeling of the data based on kinetic equations show a two-step process i.e., lateral association and elongation.

In Chapter 6, our findings are summarized and a few open questions are addressed. As an outlook, possible improvements are suggested for a working method that focuses on dealing with further problems that can be studied and encountered with vimentin processes. 


\title{
Hierarchical Architecture of Intermediate
}

Filaments

\author{
“Cytoskeletal systems are dynamic \\ and adaptable, organized more like \\ ant trails than interstate highways." \\ Bruce Alberts, Molecular Biology of \\ the Cell
}

Every eukaryotic cell has a characteristic shape and this shape can be largely attributed to the cytoskeleton. Additionally, the cytoskeleton is instrumental in the mechanics and motility of the cell. Components of the cytoskeleton contribute to a 3D network which provides rigidity and stability, much like the skeleton in human bodies. This chapter focuses on the biological relevance of our study by introducing the family of intermediate filament proteins and specifically vimentin. Firstly, concepts of the cytoskeleton in eukaryotic cells are presented with focus on intermediate filaments in general. In Sec. 2.3 , structure, function and clinical relevance of vimentin are discussed. Biochemical methods to prepare vimentin for experiments are explained in Sec. 2.4. Finally, the interaction of ions with the 


\section{Chapter 2 HIERARCHICAL ARCHITECTURE OF INTERMEDIATE FILAMENTS}

in vitro assembly processes of vimentin are discussed, where essential structural characteristics come into play.

\subsection{Organization in the Cell}

Eukaryotic cells lack a cell wall but consist of several organelles, small molecules, ions, proteins, DNA and RNA and to stabilize themselves, the cells rely on a complex system. These cells adapt in space as they grow and undergo division of structure internally to execute their respective functions of providing stability and regeneration [1]. The cytoskeleton plays a very crucial role in this re-organization of the cell which highly depends on the intricate system of three filaments. The local organization of the cell is affected in situations where the mechanical forces (both inside and outside) disrupt the activity of the regulatory factors of the filament system. In this section, we describe the underlying mechanisms involved in the cell organization and particularly the function of the cytoskeleton.

The cytoskeleton is made up of proteins which act as building blocks, which, when put together in a key fashion make up supra-molecular assemblies. A wide range of structures with diverse properties can form, based on how strategically these primary subunits of the proteins are assembled. These self-assembling organic "LEGO" pieces make up the cytoskeleton which caters to the changing needs of the eukaryotic cell. The complexity of the cytoskeleton can be attributed to its interaction with other cellular components such as motor proteins, nucleation promoting proteins, capping proteins and cross-linking and stabilizing proteins, polymerases and depolymerizing factors to name a few [3].

The cytoskeleton is organized into three groups: microfilaments MFs (actin filaments with a diameter of about 7-8 $\mathrm{nm}$ ), microtubules MTs (tubulin with a diameter of about $25 \mathrm{~nm}$ ) and intermediate filaments IFs with a diameter of about $10 \mathrm{~nm}$ [4]. A hypothetical epithelial cell with the cytoskeletal components is depicted in Fig. 2.1. MFs and MTs are mainly confined to the cytoplasm whereas IFs contain two main systems: one within the nucleus attached to the inner nuclear membrane [5], and one that is cytoplasmic, which connects intracellular junctional complexes situated at the plasma membrane with the outer nuclear membrane [2, 6]. The most important differences between the three main cytoskeletal polymers - the differences that distinguish the architecture and function of the networks they form - are their mechanical stiffness, the dynamics of their assembly, their polarity, and the type of molecular motors with which they associate [3]. 


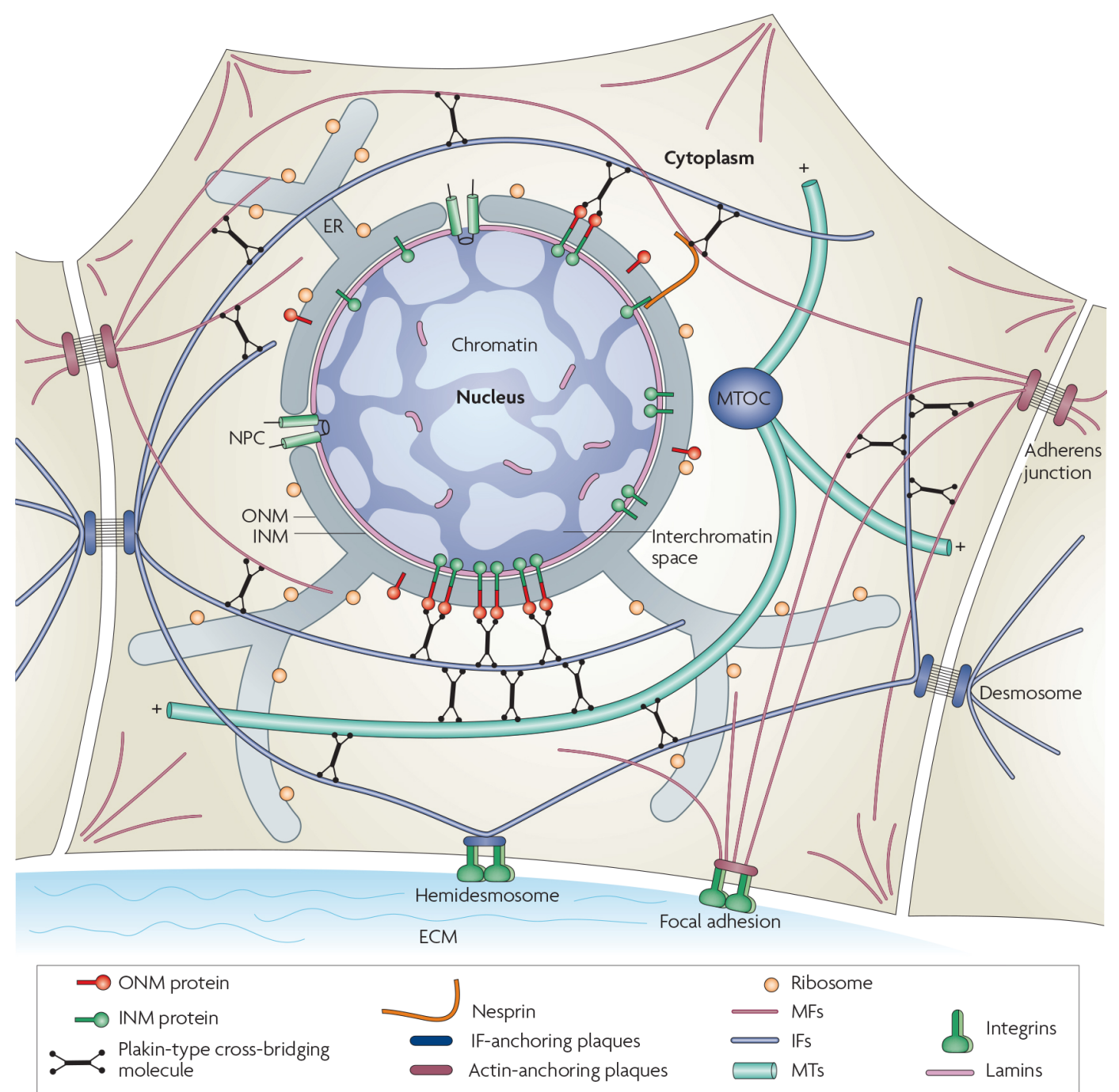

Figure 2.1.: Cytoskeletal organization in metazoan cells. A hypothetical epithelial cell is depicted with the three key systems of the cytoskeleton: microfilaments (MFs), microtubules (MTs) and intermediate filaments (IFs). They are connected to each other by several dimeric complexes. IFs are connected to the outer nuclear membrane (ONM by plectin and nesprin-3). On the inner nuclear membrane (INM), lamins (which are also IFs) attach themselves together with chromatin. This figure is reprinted from [2] with permission.

\subsection{Intermediate Filaments}

Intermediate filaments (IFs) display a wide variety of diversity in their numbers, sequences and abundance in comparison to MFs and MTs, which are very similar within cells of a particular species and between species [7]. IFs are special com- 


\section{\begin{tabular}{l|l} 
Chapter 2 & HIERARCHICAL ARCHITECTURE OF INTERMEDIATE FILAMENTS
\end{tabular}}

pared to the other members of the cytoskeleton due to their cell type specificity. There are about 70 genes in human encoding different IF proteins whereas MFs and MTs have one gene each and some isoforms. Amidst this diversity, all proteins of the IF family share a common secondary structure with a head, tail and a rod domain and a similar assembly process where a dimer is composed of two parallel $\alpha$-helical chains and intertwined in a coiled-coil rod as shown in Fig. 2.3. The dimers associate in linear arrays in anti-parallel half-staggered manner to form tetramers of the filament structure [8].

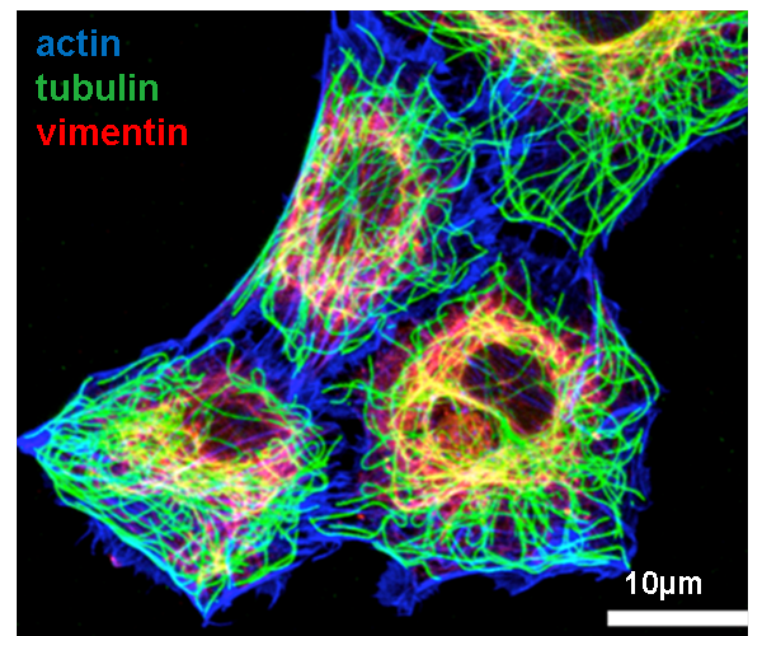

Figure 2.2.: Fluorescence microscopy image of a cell, showing the different components of the cytoskeleton. This image was obtained from Rosmarie Sütterlin and Ueli Aebi, Biozentrum University of Basel.

More detailed explanation of the hierarchical filament architecture with relevance to vimentin IF will be explained in Sec. 2.3 . Some members of the IF family are listed in Tab. 2.1. Amongst them, IFs either form homo- or hetero-polymers. Type I and II keratins form inter-type heteropolymers [9], whereas type IV IFs nestin can only form heteropolymers, with vimentin (type III IF) and $\alpha$-internexin (type IV IF) [10]. In contrast, vimentin forms homopolymers generally but can also co-polymerize with glial fibrillary acidic protein (type III IF) during diseased conditions (e.g., stroke/brain trauma and neuro-degenerative diseases) such as the induction of reactive astrocytes which are star shaped glial cells of the central nervous system [11]. 
Table 2.1.: Classification of the IF proteins into six categories [12, $13,14,15,16]$.

\begin{tabular}{llll}
\hline Type & IF name & Size $(\mathrm{kDa})$ & Distribution \\
\hline I & acidic keratins & $40-64$ & epithelial cells \\
II & neutral-basic keratins & $52-68$ & epithelial cells \\
III & desmin & 53 & $\begin{array}{l}\text { muscle } \\
\text { mesenchymal cells } \\
\end{array}$ \\
& vimentin & 54 & $\begin{array}{l}\text { (e.g. fibroblasts and smooth } \\
\text { muscle cells) }\end{array}$ \\
& & & \\
& glial fibrillary acidic & & astrocytes \\
& protein & 52 & peripheral neurons \\
& peripherin & 54 & central nervous system neurons \\
IV & neurofilaments & $61-110$ & stem cells, neuroepithelia \\
& nestin & 177 & central nervous system neurons \\
& $\alpha$-internexin & 66 & muscle \\
& synemin & 182 & all nuclei \\
V & lamins & $66-78$ & fiber cells (lens) \\
VI & filensin & 83 & fiber cells (lens) \\
& phakinin & 47 &
\end{tabular}

\subsection{Vimentin}

Vimentin is an important IF which is precariously expressed and is one of the dominant proteins of the cytoskeleton in mesenchymal cells [7]. Vimentin plays an instrumental role in developmental biology especially during the epithelialmesenchymal transition (EMT) and vice versa during embryogenesis [17]. This transition is important for organ fibrosis [18] and metastasis in cancer progression [19]. Vimentin is found in abundance in fibroblasts which is derived from primitive mesenchyme and is crucial for wound healing [20]. Recent study suggests that the presence of vimentin is sufficient to alter the shape and motility of epithelial cells to mesenchymal cells [21].

In rheology measurements, vimentin networks exhibit some unusual viscoelastic properties not shared by actin or tubulin which are crucial for cytoplasmic consistency. They are less rigid (have lower shear modulus) at low strain but harden at high strains and resist breakage, suggesting they maintain cell integrity [22].

with regard to cell rigidy, the extracellular matrix (ECM) comprises of complex three-dimensional (3D) scaffold made up mostly of collagen [2]. However, when vimentin is plated within collagen gels, a few vimentin-deficient cells exhibit reduced contraction of the gels which in-turn suggest that the contractibility of the 
cells is reduced [23]. These results are consistent with the assumption that vimentin IFs carry tensile strength which governs the mechanics of the cytoskeleton. Furthermore, cell-cell physical interactions are dramatically decreased in vimentin-deficient cells, when compared to wild-type cells. These results suggest that vimentin IFs are important for maintaining mechanical interactions between cells, possibly by carrying some of the contractile forces generated among cells [24].

Katsumoto et al. reports a visualization of the 3-D relationship between vimentin IF and the organelles of the cell. Vimentin is observed to be present very close to the cell organelles such as nucleus, endoplasmic reticulum and mitochondria and attached to these organelles laterally or terminally, thus supporting the location of the organelles in the cell cytoplasm [25]. In addition to providing support to these organelles in the cytosol, vimentin IFs are instrumental in maintaining cell integrity. Goldman et al. show in an in vivo study, cells without vimentin are delicate and prone to collapse when disturbed by a micro-puncture [26].

\subsubsection{Structure}

Vimentin, like other IF proteins, consists of an N-terminal "head" domain, a central rod and a C-terminal "tail" domain and assembles hierarchically [7, 27] as shown in Fig. 2.3. Dimers are fundamental subunits of vimentin assembly and are formed through interaction of two rod domains which are expressed cotranslationally to form a coiled-coil dimer [28]. These dimers assemble in an antiparallel fashion to form tetramers which then assemble into unit length filaments (ULFs) composed typically of eight tetramers which are approximately $17 \mathrm{~nm}$ in diameter and $60 \mathrm{~nm}$ in length. End-to-end annealing processes of the ULFs occur, which has been demonstrated by studies of kinetic and mathematical modelling, immunofluorescence, and total internal reflection fluorescence microscopy (TIRFM) [29, 30, 31, 32]. This process is crucial in the assembly and elongation of the vimentin filaments. Further, radial compaction occurs in the higher order filaments which reduces the diameter to approximately $10 \mathrm{~nm}$. These mature vimentin filaments act as semi-flexible polymers which have a persistence length of $2 \mu \mathrm{m}$ [33].

At low ionic buffers such as $2 \mathrm{mM}$ phosphate buffer (PB), the population of vimentin consists of tetramers. Upon the addition of monovalent salts such as potassium chloride $(\mathrm{KCl})$ or sodium chloride $(\mathrm{NaCl})$, tetramers undergo an assembly process to form filaments [34, 35, 36]. In contrast, addition of divalent salts such as 
a)

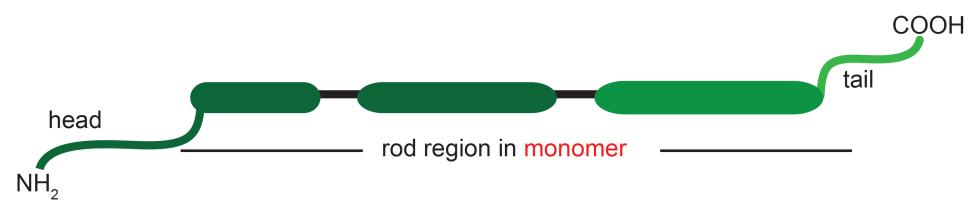

b)

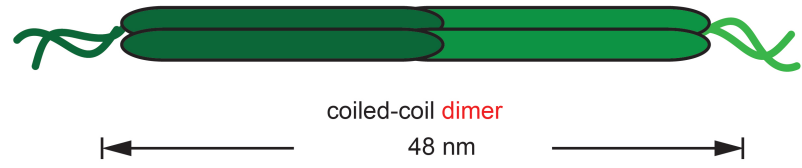

c)

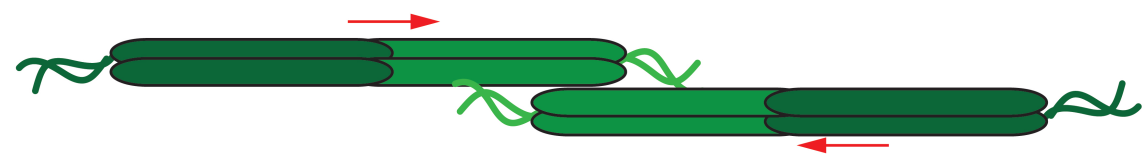

d)

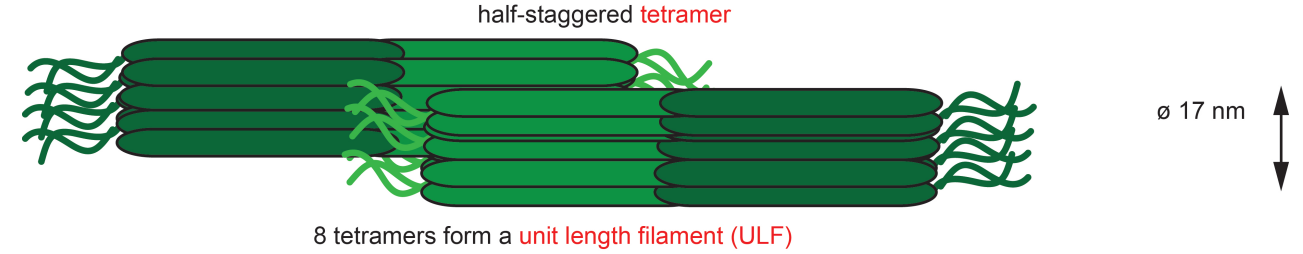

e)

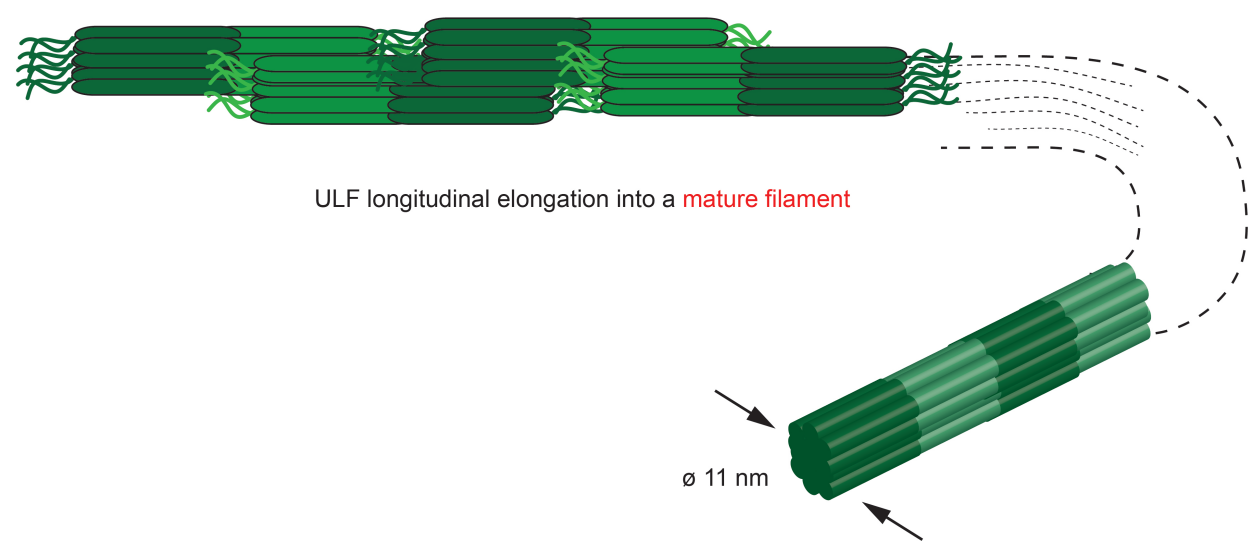

Figure 2.3.: Sketch of the hierarchical structure of vimentin assembly. a) The vimentin monomer has a head, tail and a rod domain. The head and the tail regions are skipped in all the follwing steps of the sketch. b) At low ionic conditions, the monomers form dimers which then associate in a half-staggered fashion to form tetramers as shown in c). d) Upon the addition of monovalent ions, usually eight tetramers laterally associate to form a unit-length filament (ULF). e) ULFs longitudinally anneal to form a filament which undergoes a radial compaction step to a form mature filament.

magnesium chloride $\left(\mathrm{MgCl}_{2}\right)$ and calcium chloride $\left(\mathrm{CaCl}_{2}\right)$ plays a distinct role in the size and surface characteristics of vimentin filaments [37, 38, 39]. It is shown 


\section{Chapter 2 HIERARCHICAL ARCHITECTURE OF INTERMEDIATE FILAMENTS}

that vimentin assembly is slowed down in the presence of divalent ions like magnesium and calcium whereas in the presence of excess monovalent ions like potassium and sodium, the surface and nanostructure is affected in a controlled way [38, 40]. Structural properties of vimentin filaments are found to be different in comparison to the filaments assembled only in the presence of potassium ions and filaments assembled only in the presence of magnesium ions [37]. However so, vimentin assembly and aggregation are dynamic processes and there is a steady state exchange of vimentin subunits between a soluble pool and filaments [41], whereby subunit exchange occurs along the length of the filament at a much slower pace [29, 42].

Bundling and network formation of vimentin filaments play a crucial role in the mechano-stability of the cytoskeleton [43]. In situ, the assembled vimentin filaments are influenced by the divalent ions to build cross-linked networks which depend on the protein concentration. Micro-rheology experiments reveal that the magnesium ions act as effective cross-linkers for the pre-assembled vimentin filaments and facilitate the stiffening of the networks [44, 45, 46]. Bundle formation of vimentin is mediated by multiple positive charges indicating the role of charged ions in the light scattering experiments [47].

In summary, in vitro techniques combined with high-resolution techniques help us to understand the details of the kinetic processes of vimentin and the influence of ions on its structure.

\subsection{Purification and Reconstitution of Vimentin}

Human vimentin protein is recombinantly expressed in Escherichia coli (E. coli) bacteria which renders a high protein yield and purity making it a desirable form of expression system [48]. Purification of vimentin after the initial expression stage is from inclusion bodies [6]. It is, however, of utmost importance to perform Xray scattering experiments with purified protein samples at concentrations in the range of $\mathrm{mg} / \mathrm{ml}$ to render structural information at a high signal-to-noise ratio [49]. For the X-ray experiments, a concentration of $\approx 1-4 \mathrm{~g} / \mathrm{L}$ is used and for the light scattering experiments, a much lower concentration of $\approx 0.09-0.06 \mathrm{~g} / \mathrm{L}$ is used

Expression and purification of vimentin in E.coli bacteria is performed in-house by Susanne Bauch. The plasmid for the transfection is provided by Harald Herrmann, DKFZ Heidelberg, Germany. Unless indicated otherwise, all chemicals are from 
Carl Roth GmbH+Co. KG (Karlsruhe, Germany). This section explains the several steps of a reproducible protocol for vimentin production which is adapted from [50]. Details of this protocol are given in Appendix. A

- E. coli bacteria are transfected with a plasmid encoding the human wild type vimentin and cultivated to express the protein

- The protein is purified from the bacterial solution by several centrifugation, homogenization and washing steps in different buffers and two ionexchange chromatography columns which leads to typical protein concentrations in the range of $3-4 \mathrm{mg} / \mathrm{mL}$

- The purity of the vimentin is controlled by an Sodium dodecyl sulfate (SDS) page gel

- The purified vimentin can be stored in urea solution at $-80{ }^{\circ} \mathrm{C}$ for several months until use

\subsubsection{Dialysis and Assembly Techniques}

The stored vimentin has to be renatured into tetramers prior to the experiments. This is performed by dialyzing the vimentin against $8 \mathrm{M}$ urea for 30 minutes and then in a stepwise manner at 4,2, and $1 \mathrm{M}$ urea for 30 minutes each at room temperature. Finally, an overnight dialysis into $2 \mathrm{mM} \mathrm{PB}$ is performed at $4{ }^{\circ} \mathrm{C}$. All dialysis steps are performed using membranes of $50 \mathrm{kDa}$ cut-off (Spectra/Por 7, Spectrum Europe B.V., Breda, Netherlands) which is lower than the vimentin monomer weight of $54 \mathrm{kDa}$. All solutions for dialysis are prepared using $2 \mathrm{mM}$ $\mathrm{PB}, \mathrm{pH}$ 7.5. Before the use in experiments, the protein concentration is determined by measuring the absorption at $280 \mathrm{~nm}$ with UV-Vis spectroscopy and is explained in detail in Sec. 2.4.2. The dialyzed vimentin can be stored upto a week at $4{ }^{\circ} \mathrm{C}$ [6]. In principle, assembly of vimentin can be initiated either by dialysis or in Eppendorf cups with the "kick-start" mode for the vimentin aggregation studies involving divalent salts. Vimentin filaments have a varying number of tetramers per cross-section (ranging from 4-13 tetramers with the standard configuration of 8 tetramers [34, 40]) and this polymorphism is larger when the in vitro assembly is initiated in a "kick-start" mode as compared to the slower method of dialysis. Since SAXS averages out the scattering signal over several intensities arising from polymorphic samples, the "kick-start" method is employed in all our experiments which involves mixing the freshly dialyzed vimentin with $200 \mathrm{mM} \mathrm{KCl} / 2 \mathrm{mM}$ 


\section{\begin{tabular}{l|l} 
Chapter 2 & HIERARCHICAL ARCHITECTURE OF INTERMEDIATE FILAMENTS
\end{tabular}}

$\mathrm{PB}$ in a 1:1 ratio in an Eppendorf cup for 15 hours at $\mathrm{pH} 7.5$ and temperature of $37^{\circ} \mathrm{C}$.

For the studies of vimentin assembly, microfluidic manipulation techniques are used to initiate the process such as diffusive mixing of $100 \mathrm{mM} \mathrm{KCl}$ to the tetrameric vimentin in continuous flow devices as explained in Sec. 3.4.1 and recirculative flow of the droplets after pinch-off which is explained in Sec. 3.4.2. For the assembly experiments of light scattering, vimentin is diluted to the desired concentration and filled in cuvettes. Different concentrations of $\mathrm{KCl}$ are mixed into the cuvette containing the vimentin with a syringe and manual shaking is performed before the time-resolved measurements are performed.

Quality of the dialyzed vimentin can be further examined by assembling it into filaments and checking with several techniques such as confocal fluorescence microscopy, electron microscopy (EM) and atomic force microscopy (AFM). A high resolution image can be obtained with these techniques which shed light on the filament morphology in detail. Homogeneously spread, well-formed filaments are observed in the region-of-interest which are devoid of any form of aggregates as shown in Fig. 2.4. This indicates that the vimentin produced for experiments is of high and usable quality.
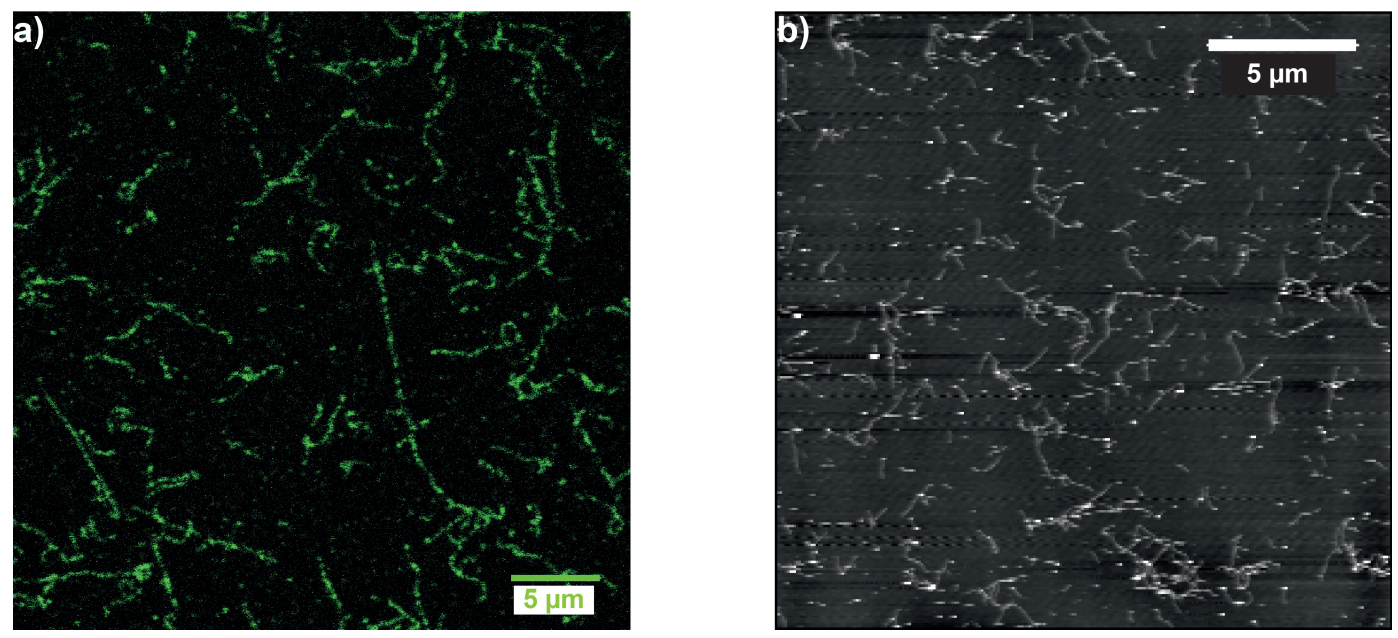

Figure 2.4.: Confocal fluorescence microscopy and AFM images of vimentin filaments.a) Vimentin wild type mixed with vimentin labeled with AlexaFlour 532 dye shows filaments assembled in Eppendorf cups after 15 hours b) The same batch of wild-type vimentin as used in a) without any labeled dye. Both images were taken by Viktor Schroeder. 


\subsubsection{Estimation of Protein Concentration by UV-Vis Spectroscopy}

In order to reliably perform X-ray and light scattering experiments, it is essential to have a knowledge of the initial vimentin concentration. For this purpose, we perform UV-Vis spectroscopy measurements on the commercially available NanoDrop ND-1000 spectrophotometer (NanoDrop ${ }^{\mathrm{TM}}$ Technologies, Wilmington, USA) where protein concentration can be deduced with a sample volume of $2 \mu \mathrm{L}$. The light absorbance $A$ is recorded at a wavelength of $280 \mathrm{~nm}$ :

$$
A=\log \left(\frac{I}{I_{0}}\right)=\epsilon l_{p} c
$$

Here, the extinction coefficient for vimentin is about $\epsilon=24.24 \times 10^{3} \mathrm{M}^{-1} \mathrm{~cm}^{-1}$ [38]. The optical path $l_{p}$ is considered to be $10 \mathrm{~mm}$, the molecular weight of vimentin monomer is $53,67 \mathrm{kDa}$ [34] and $c$ is the protein concentration. Errors can occur in the determination of the extinction coefficient $\epsilon$ and some of the common factors are buffer type, ions and denaturants. Not only that, variations in the optical path length and the incident intensity can be additional factors for an intrinsic error of the measuring concentration. Therefore, the mean of at least three measurements can be considered for determining the final protein concentration.

\subsection{Role of Ions in Assembly Process}

Vimentin assembly is a half-staggered complex forming hierarchical mechanism which is instantaneously initiated by the presence of monovalent ions, whereas, the other cytoskeletal components actin filaments or microtubules grow end-toend by the addition of single subunits using ATP or GTP, respectively [31]. For a certain concentration of the added monovalent salt, the ionic bond that leads to assembly between the head group of the vimentin IF and the monovalent ions is mediated by electrostatic interactions [51].

According to the amino acid sequence of vimentin protein monomers, the emerging filaments are highly negatively charged polyelectrolytes which interact strongly with counter ions. Such charge interaction can also play a role in physiological settings and in order to gain a better principle understanding of the processes involved, a number of different experimental techniques have been employed. Macro- and microrheology measurements show that already low concentrations $(\approx 2 \mathrm{mM})$, of divalent $\mathrm{Mg}^{2+}$ crosslink filaments in vimentin networks [44, 45, 46] and higher ion concentrations even lead to a strong aggregation effect visible di- 


\section{\begin{tabular}{l|l} 
Chapter 2 & HIERARCHICAL ARCHITECTURE OF INTERMEDIATE FILAMENTS
\end{tabular}}

rectly by fluorescence microscopy, where the individual filaments "zip" together within minutes [52, 53, 54]. In addition, nanoscale methods like atomic force microscopy (AFM), electron microscopy (EM), small angle x-ray scattering (SAXS), and transient electric birefringence (TEB) have been used to investigate the influence of different ion species and concentrations on the individual filaments, or even subunits thereof. The studies show that the results depend on a variety of parameters like buffer conditions and origin of the protein (tissue or recombinant). Thus, vimentin assembled in the presence of $\mathrm{MgCl}_{2}$ instead of monovalent salt looks the same when imaged by EM [39]. TEB, by contrast, reveals largely disordered filaments in the presence of magnesium that are shorter and thicker than usual [38]. SAXS is sensitive to the internal structure of the assemblies [55] and shows that both the ion species and the ion concentration influence the resulting filament [37]. 


\title{
Microfluidics for in situ Investigation of Bio-Processes
}

\author{
"One man is equivalent to all \\ creation, one man is a world of \\ miniature"
}

Albert Pike

Microfluidics exploits its obvious 'size' to manipulate, control and mix fluid volumes on the order of nanolitres, in channels with dimensions of tens to hundreds of micrometers. Microfluidics has offered major applications in the study of fast reaction kinetics [56], rapid screening of protein crystallization [57] and droplets as microreactors for single cell studies [58].

This chapter introduces the basic concepts of behavior of fluids at the microscale and focuses on the two different methods of fluid manipulation i.e., continuous flow and droplet-based microfluidics. The challenges of combining microfluidics and small angle X-ray scattering (SAXS) are discussed, coupled with the respective fabrication processes for the two methods. Results from finite element method (FEM) simulations complement the experimental technique and give us insights 


\section{\begin{tabular}{l|l} 
Chapter 3 & MICROFLUIDICS FOR IN SITU INVESTIGATION OF BIO-PROCESSES
\end{tabular}}

into control of species (i.e., protein and salt in our work).

\subsection{Theory}

In this section, some of the essential concepts governing transport and mixing in microflow are introduced, such as the dimensionless quantity "Reynolds number" and the diffusion equations.

\subsection{1 "Life at Low Reynolds Number" [59]}

In fluid mechanics, we usually view fluids as "continuous" and discuss "average" fluid properties rather than considering the properties of each molecule, despite the fact that properties like velocity and density vary differently at the molecular length scale. This continuum approximation is developed for macroscale fluid mechanics, but is important in this context because it allows us to analyze microfluidic flows with the same governing principles.

Consider a fluid flowing in a microfluidic channel with density $\rho$ and constant viscosity $\eta$. The Navier-Stokes equation for such an incompressible Newtonian fluid takes the differential form [60]:

$$
\rho\left(\frac{\partial \vec{v}}{\partial t}+(\vec{v} \nabla) \vec{v}\right)=-\nabla P+\eta \Delta \vec{v}+\vec{f}
$$

where $\vec{v}$ is the velocity field, $P$ is the pressure, $\eta$ is the dynamic viscosity and $\vec{f}$ is the applied external force per volume e.g., gravity. However, by introducing some scaling factors, the Navier-Stokes equation can be non-dimensionlized. Nondimensionlization eases the analysis of the problem under study and summarizes them into one parameter. In addition to reducing the number of parameters, the non-dimensionalized equation helps to gain a greater insight into the relative importance of various terms present in the equation. For the case of flow without heat transfer, the non-dimensionalized Navier-Stokes equation depends only on the Reynolds number $(R e)$ and hence all physical parameters such as channelwidth of the related experiment will have the same value of non-dimensionalized variables for the same $\operatorname{Re}$ [61].

By adapting the dimensionless variables for space $\overrightarrow{x^{*}}\left(=\frac{\vec{x}}{|L|}\right.$, where $L$ is the typical length scale of the system) and velocity $\vec{V}^{*}\left(=\frac{\vec{v}}{|V|}, V\right.$ is the typical velocity), we obtain time $\tau=\frac{t V}{L}$ and pressure $P^{*}=\frac{P L}{\eta U}$ ( $U$ is the initial velocity) where vis- 
cous forces are dominant. Therefore, the non-dimensionalized form of the NavierStokes equation can be rewritten as:

$$
\operatorname{Re}\left(\frac{\partial \overrightarrow{V^{*}}}{\partial \tau}+\vec{V}^{*} \cdot \nabla^{*} \overrightarrow{V^{*}}\right)=-\nabla^{*} P^{*}+\Delta^{*} \vec{V}^{*}
$$

where $R e=\frac{\rho U L}{\eta}$ is the Reynolds number, a dimensionless quantity arising from the ratio of the inertial forces to the viscous forces in flow. Eqn. 3.2 holds good in the absence of a free surface where any external forces are neglected for computational simplicity.

The Reynolds number, though dimensionless, always governs the transition from the laminar flow region to turbulence. Because $\operatorname{Re} \propto L$, the small dimensions of microfluidic channels are responsible for very low $R e$, resulting in laminar flow. In fact, for most microfluidic applications $R e \ll 1$. For a person swimming in water Re would be on the order of $10^{4}$, for a small fish it can be as low as $10^{2}$. But at the microscopic scale, for something like a paramecium or a bacteria, the $\mathrm{Re}$ of water will further be lower on the order of $10^{-4}$. At that scale, viscous forces are dominant and inertia does not play a role. According to Purcell [59] "At $R e \ll 1$, time doesn't matter. The pattern of motion is the same, whether slow or fast, whether backward or forward in time". This highly felicitates laminar flow which is controllable and as a consequence gives a simplified version of Eqn. 3.1

$$
\nabla P=\eta \Delta \vec{v}
$$

This is called the Stokes-Equation and indicates that for a given flow, the inertial forces are small compared to viscous flow and any evident turbulence is suppressed by friction. This kind of flow is also called creeping flow. This physical phenomenon evokes two additional interesting properties in incompressible Newtonian fluids:

- Instantaneity: a Stokes flow has no dependence on time other than through time-dependent boundary conditions;

- Time-reversibility: a time-reversed Stokes flow solves the same equations as the original Stokes flow. Practically, it implies that it is difficult to mix two fluids using creeping flow i.e., ignoring the inertial term in Eqn. 3.1

This quality makes microfluidics a desirable tool due to the laminar flow, as opposed to turbulent flow which is of stochastic nature (which might attain random 


\section{\begin{tabular}{l|l} 
Chapter 3 & MICROFLUIDICS FOR IN SITU INVESTIGATION OF BIO-PROCESSES
\end{tabular}}

values).

\subsubsection{Diffusion in Microsystems}

While laminar flow is advantageous in several microfluidic applications, it can be a nuisance where mixing of different solutes is required. Since biological and chemical assays need either mixing or dilution at some point, several smart techniques have been developed such as adaptation in the geometric design or exploitation of the enhanced diffusivity at small dimensions. To fully understand the mixing and distribution of a solute in flow, two processes have to be addressed: convection and diffusion.

Consider a solute molecule with a diffusion coefficient $D$, traveling a distance $L$ in a three-dimensional space over time $t$. If the solute molecule travels from one point to the other with a local velocity $V$, it gives us the convection rate $\sim L^{2} / D$ and the diffusion rate $\sim L / V$ as illustrated in Fig. 3.1. The ratio of these quantities is a dimensionless number which is extremely important in convection-diffusion problems and is defined as the Péclet number $(\mathrm{Pe})$ :

$$
P e=\frac{\text { convective transport rate }}{\text { diffusive transport rate }}=\frac{L^{2} / D}{L / V}=\frac{V L}{D}
$$

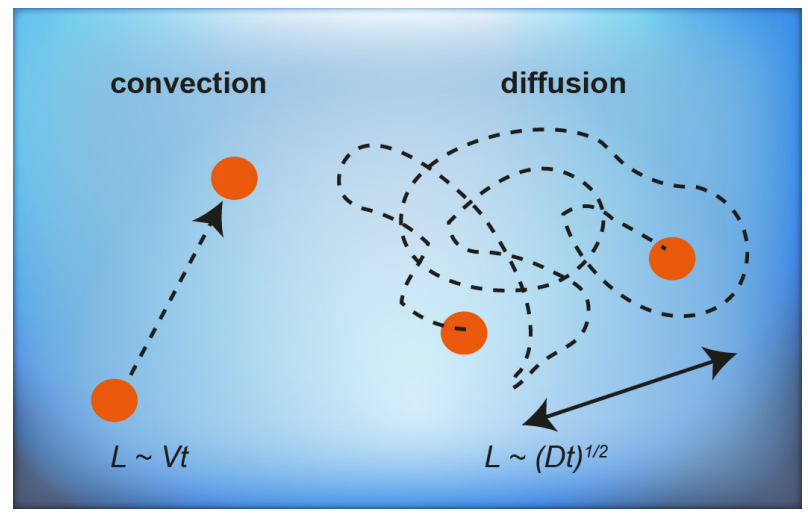

Figure 3.1.: Sketch showing a difference between convection and diffusion.

In our work, we need to mix different solutes in our microflow and realize the localized effect of the mixing and distribution at each point at constant $L$. With this in mind, few questions arise when dealing with the convection-diffusion problems:

-What effect dominates in microfluidic channels? Is it convection or diffusion? 
- How can the mixing time of two or more fluids be increased/reduced?

In order to arrive at a conclusive argument, we first consider the question of mixing in microsystems where Pe plays a role due to the effect of both, the diffusion and convection time. To illustrate the significance of this, let us consider an example of fluorescein mixed with water in a channel of width of $100 \mu \mathrm{m}$, flowing at a rate of $30 \mu \mathrm{m} / \mathrm{s}$. For this $P e \approx 10$ [60], which suggests that the diffusion is slower than convection. However, diffusion dominates when the Pe numbers are small and if the numbers are large, convection dominates. In such a case, the microfluidic channels have to be scaled in physical dimensions appropriately. However, in a channel of $L \approx 0.1 \mathrm{~mm}$ and fluid velocity $V$ at $\approx 0.1 \mathrm{~mm} / \mathrm{s}, \mathrm{Pe}$ is $10^{3}$ for a protein of diffusion coefficient $10^{-11} \mathrm{~m}^{2} / \mathrm{s}$ and 10 for a small ion of diffusion constant $10^{-9} \mathrm{~m}^{2} / \mathrm{s}$. This brings us to the conclusion that mixing in microsystems is heavily influenced with the effect of both convection transport (in case of the protein in a channel) as well as the diffusive transport (in case of a ion distribution) of molecules.

\subsection{Microfluidic Fabrication Techniques}

\subsubsection{Photolithography}

The first step in the process of producing usable microfluidic devices for our studies is the drawing of the channel designs with AutoCAD software (Autodesk, München, Germany). The resulting designs are then printed as emulsion film on polyester (Selba S. A., Versoix, Switzerland). These structures are printed with a sufficiently high resolution for features with a minimum size of about $5 \mu \mathrm{m}$. The photolithography step is common to both continuous flow and droplet-based devices.

Following this, "master wafers" are fabricated with photo-lithographic techniques in the clean room of ISO-5 standard which maintains a low-level of external contaminants such as dust particles, chemical vapors and bio-pollutants. For this purpose, we use a 2-inch silicon wafer on which the design is transferred. It is first rinsed with iso-propanol and dried with dry nitrogen and again dried at $200{ }^{\circ} \mathrm{C}$ on a hot plate for $10 \mathrm{~min}$. Then, SU-8 3050 negative photo-resist (MicroChem, Newton, USA) is spin coated onto the silicon wafer (spin speed varies according to the channel height needed which is calculated from the SU-8 3000 datasheet [62]). After spin coating, the wafer with a layer of resist is placed on a hot plate at a tem- 

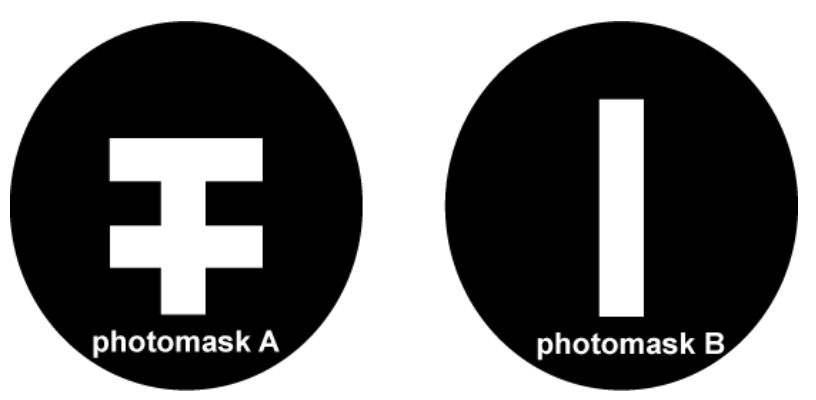

Figure 3.2.: Representative sketch of the photomasks

perature of $95^{\circ} \mathrm{C}$ in a soft bake step. Using a mask aligner (MJB4, Süss MicroTec AG, Garching, Germany), the printed photomask is brought into vacuum contact with the coated wafer and is exposed with UV-light at a wavelength of $365 \mathrm{~nm}$ in regions where the mask is transparent as shown in Fig. 3.2. The exposure time is chosen with respect to the resist type and height. The exposed resist is baked in a post exposure step again with respect to the resist type. During this process the exposed regions in the resist are cross-linked. Finally, the wafer is developed in SU-8 developer (MicroChem) for a few minutes until the non-crosslinked resist dissolves completely. The "master-wafer" is rinsed again with isopropanol and dried with dry nitrogen rendering it free from any further moisture. The channel height is measured after its production using a profilometer 'Dektak 6' (Veeco Instruments Inc., Mannheim, Germany).

For the fabrication of devices with several layers, where a second layer has to be deposited onto the first layer, steps from spin coating until post exposure baking have to be followed. The mask to be used must have a similar design to the first layer and can be aligned onto the second layer with an on-axis microscope as shown in Fig. 3.2 .

\subsubsection{Soft lithography}

Once the "master-wafer" is fabricated, it can be used to make several copies of the channel design using soft-lithographic techniques. For this purpose, we first coat the freshly fabricated wafer with a few drops fluorosilane ((heptafluoropropyl) trimethylsilane $97 \%$, Aldrich) in a desiccator, which renders the surface of the silicon master hydrophobic. Then the wafer is molded with PDMS mixed with crosslinker at a ratio of 10:1 (Silgard 184, Dow Corning GmbH, Wiesbaden, Germany). The wafer with raw PDMS is baked at $65{ }^{\circ} \mathrm{C}$ in an oven for at least one hour. This step is common to both continuous flow and droplet-based devices. 


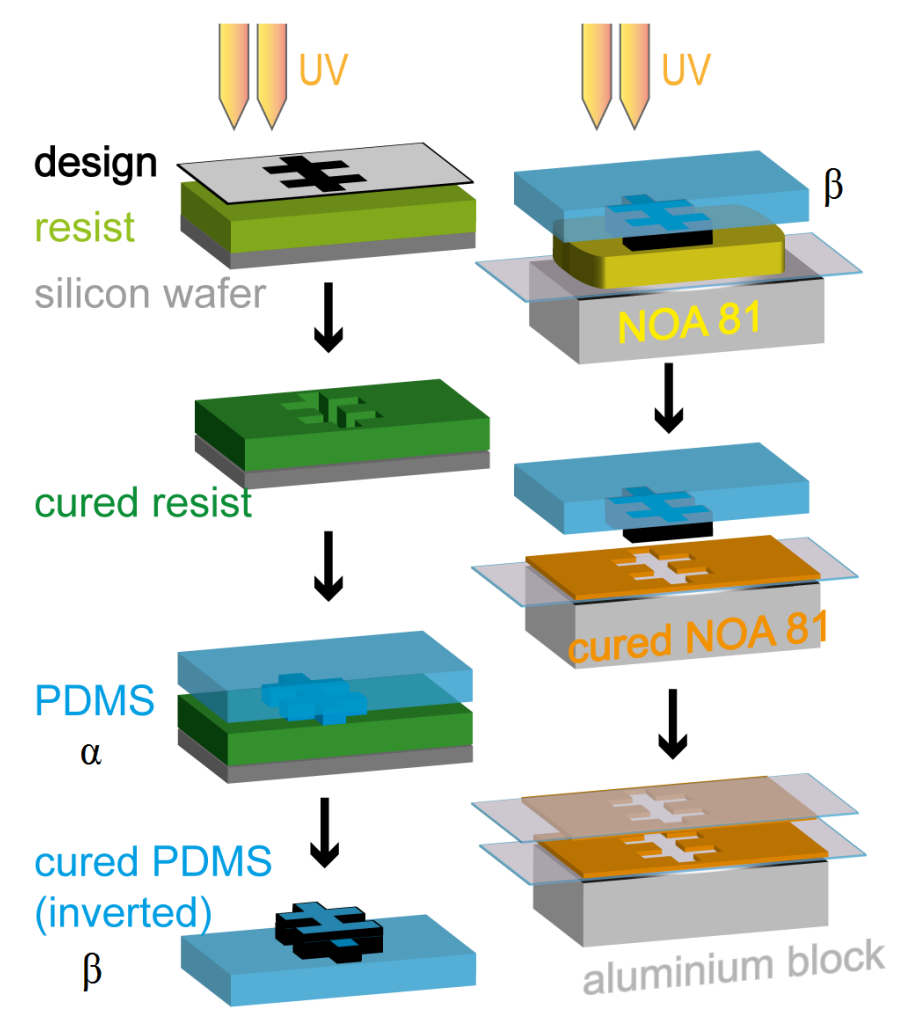

Figure 3.3.: Schematic diagram of device fabrication. Step-by-step schematic of the device fabrication. Silicon wafers are structured by standard photolithography. These wafers are utilized for fabricating PDMS stamps. Finally, the stamps are pressed into UV-curable glue (Norland Optical Adhesive, NOA 81). Two identical copies of structured NOA-81 are produced, aligned and bonded. Figure taken from [63] with permission.

Specific Steps for Continuous Flow Devices

Now, the first PDMS $(\operatorname{stamp} \alpha)$ is a negative copy from the wafer which has positive structure. However, to be able to produce an X-ray compatible device which are used in the experiment, a positive PDMS (stamp $\beta$ ) has to be fabricated and the chronology of the steps in described in Fig. 3.3. For this, we first mark the edges of PDMS $\alpha$ stamp with the black ink which will later facilitate us to effectively get the second PDMS $\beta$ out. PDMS stamp $\alpha$ is plasma cleaned for $30 \mathrm{~s}$ in the PDC-32G-2 plasma cleaner (Harrick Plasma, Ithaca, New York, USA) to make the surface charged. Immediately, PDMS stamp $\alpha$ is vapor coated with HMDS (hexamethyldisilazane, Fluka, Germany) for $10 \mathrm{~min}$ in the desiccator under the fume hood. Now, a mixture of PDMS with crosslinker at a ratio of 10:1 is poured onto 


\section{\begin{tabular}{l|l} 
Chapter 3 & MICROFLUIDICS FOR IN SITU INVESTIGATION OF BIO-PROCESSES
\end{tabular}}

the PDMS stamp $\alpha$ by pushing it down. This is then degassed using a vacuum pump on the desiccator until all the air-bubbles are removed. It is again baked for $65{ }^{\circ} \mathrm{C}$ in an oven for at least one hour and the stamp $\beta$ can later be cut out.

The now fabricated, PDMS stamp $\beta$, can be used to make a thin, X-ray compatible device from the UV-curable NOA-81 glue (Norland Optical Adhesive, Norland, Cranberry, NJ, USA) . Firstly, 'Tesa' adhesive tape (tesa Pack, tesa SE, Hamburg, Germany) is attached (which is bigger than the stamp in proportion) to flat a aluminum block. It helps to have the surface completely even and to peel the cured NOA-81 easily. $100 \mu \mathrm{l}$ NOA-81 is poured on PDMS stamp $\beta$. All existent airbubbles are removed with a pointy pipette tip. $180 \mu 1$ NOA-81 is then poured on the adhesive tape and all air bubbles are eliminated again. Now, the stamp $\beta$ is carefully pressed onto the NOA- 81 on the tape and exposed to UV-light ( $8 \mathrm{~W}$ source) for $1 \mathrm{~min}$. This cures the NOA- 81 glue and the stamp can be removed. This step is repeated again to get a second structure of NOA-81. Both the NOA-81 structures are now aligned on top of each other under the stereo-microscope and finally exposed to UV light for at least $10 \mathrm{~min}$.

The resulting ensemble of a device is glued onto a plain PDMS support stamp with the fast curing 'Loctite' glue (two component: Loctite 406 and 770, Henkel, Düsseldorf, Germany). Holes are then punched into the device for tubing connection with a puncher of diameter $=0.75 \mathrm{~mm}$. The open holes on the NOA-81 side are sealed with Kapton tape. Polyethylene tubing (Intramedic ${ }^{\mathrm{TM}}$ PE20, BD, Franklin Lakes, USA; inner diameter of $380 \mu \mathrm{m}$ ) is inserted into the punched holes and they self-seal as the outer tubing diameter $(1.09 \mathrm{~mm})$ exceeds the punch diameter. However, to avoid and minimize the risk of leakage, loctite glue can be used on tubing connection to seal before the experiment.

The final channel height of the 3D channeled microfluidic device is about $240 \mu \mathrm{m}$ and the total sample thickness is about $500 \mu \mathrm{m}$. All channels are $300 \mu \mathrm{m}$ wide. The main flow direction is the $x$-direction, $z$ is the height and $y$ is the width of the channel. In this case, the origin is located at the center of the main channel where the "step" is positioned at $x=y=z=0$ as discussed in Sec. 3.4.1. 


\section{Assembling Process of Droplet-Based Devices}

There are two utilities of the droplet-based devices in our experiments: for X-ray experiments and for optical microscopy experiments. Both types of devices follow the same methods for photolithography and soft-lithography, except each has an extended material compatible (i.e., PDMS-glass for microscopy and PDMS-Quartz capillary for X-ray experiment) and are explained in detail in the following subsections.

For the X-ray experiments, the resulting PDMS $\alpha$ stamp from soft-lithography is sufficient where holes of $0.75 \mathrm{~mm}$ are punched for the insertion of tubing. This device and another layer of plain PDMS are surface-treated in an air plasma cleaner (Harrick Plasma, Ithaca, USA) and bonded as they are brought into contact afterwards. To enable the insertion of the $100 \mu \mathrm{m}$ capillary into the PDMS, it is severed off at one end.

Since these devices are only a part of the composite ensemble, care has to be taken to achieve a channel height of $100 \mu \mathrm{m}$ on the PDMS stamp already during photolithography. If not, there is a possibility that the capillary of $100 \mu \mathrm{m}$ outer diameter is unable to penetrate inside the channels which often leads to snapping of the capillary or dislocation of the channels while plasma-bonding. The $100 \mu \mathrm{m}$ circular cross-section capillary is carefully inserted into the square cross-section of the PDMS under the microscope avoiding the point of droplet generation. Freshly prepared raw PDMS is used to seal the junction area around the capillary and baked in the oven at $65{ }^{\circ} \mathrm{C}$ for about an hour. The fully-functional assembled device is shown in Fig. 3.4 .

For the optical microscopy experiments, the resulting PDMS stamp from initial soft-lithography step is punched with holes $0.75 \mathrm{~mm}$ diameter for the insertion of tubing. For the sealing of the channels, a glass cover slip is used. The PDMS and the glass cover slips are thoroughly washed with iso-propanol and dried with dry nitrogen. Further evaporation of remaining liquid is done by placing them on the hot-plate for $3 \mathrm{~min}$ at $60{ }^{\circ} \mathrm{C}$. Subsequently, the PDMS as well as the cover slip are placed in an oxygen plasma (PDC-32G-2, Harrick Plasma, Ithaca, US) and afterwards pressed together to form a covalent bond. The fully-functional device for microscopy experiments is presented as a image in Fig. 3.5 


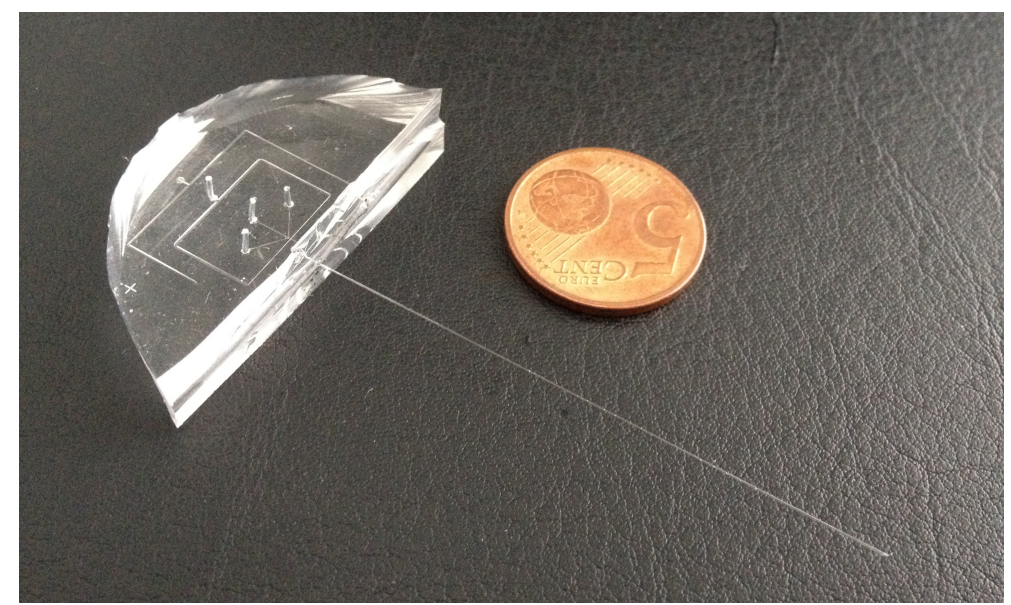

Figure 3.4.: Photograph of the assembled droplet device. The composite PDMS-Quartz device is placed with a 5-cent coin to highlight its size and portability.

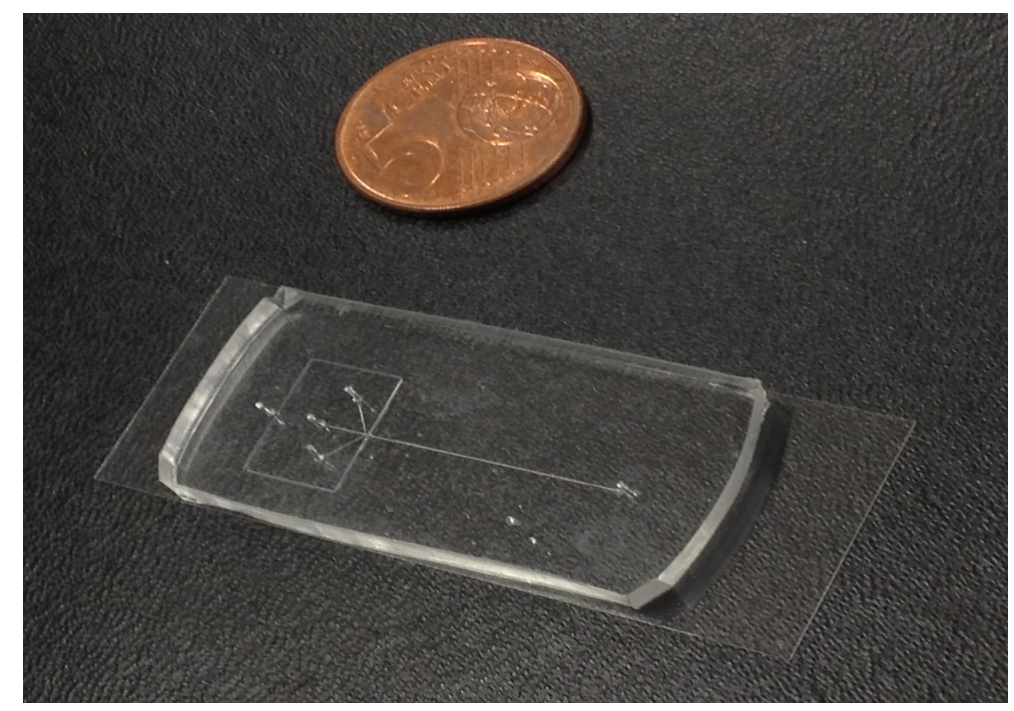

Figure 3.5.: Photograph of the assembled droplet device for optical microscopy

\section{Ombrello Coating}

An important treatment of the microfluidic devices for droplet generation is the flushing of the channels with 'ombrello' (Autoserv, Sinzheim, Germany). Ombrello is assumed to bind to the channel walls and render them hydrophobic ensuring that the oil phase of the droplet emulsion wets the channel walls. This step is performed for every device to be used before the actual measurement. Once the ombrello is flushed in, within the next seconds, it is removed by blowing air into the channels since ombrello has a tendency to solidify when exposed to air quickly. 
The device can be used for measurement within one week of the ombrello-coating, however, it is advisable to coat and use immediately.

\subsection{Motivation for Device Geometries}

Before describing the fabrication techniques involved in the production of microfluidic devices, it is essential to discuss the motivation of the geometries used for the experiments presented in this thesis. There are two main devices which are explained in detail in the forthcoming sections: continuous flow experiments (see Sec. 3.3.1) and droplet experiments (see Sec. 3.3.2).

\subsubsection{Continuous Flow Microfluidics}

One of the approaches of the microfluidic mixers and devices is the implementation of the continuous flow experiments. The advantages of the continuous flow experiments is to be able to track the progress of flow reaction at discrete points and determining the initial rates of fluid flow of different species such as proteins and salts and convolution values long the flow direction. Protein adhesion to channel surface could be a potential problem in these continuous flow experiments due to restriction in flow and clogging. Hence we have incorporated a "step" which is a three-dimensional structure on the channel and avoids protein adhesion of vimentin.

Small-scale mixing of several components is achieved in microfluidic channels in bio and chemical analyses. Often, the mixing is triggered with mechanical, electrical and magnetic forces [64]. For a fluid system with low-diffusivity, the reaction times might take very long and hence flow focusing with several channels can accelerate the fluid mixing.

In our experiments, we strive for "diffusive-mixing", which can be achieved by reducing the the mixing path and increasing the contact between the two solutions. In the work presented in this thesis, there is a single long channel acting as the central inlet for the sample flow while the solvent streams join through adjacent inlets and form the sheath flow as shown in Fig. 3.6. With this kind of hydrodynamic focusing, the width of the sample fluid (tetrameric vimentin here) can be controlled and focused to a narrow stream. The advantage of the step in the reported design is discussed in [35] and is dealt in-detail in Sec. 3.4.1. where the central stream is engulfed by the incoming sheath streams in all directions thus avoiding the contact and reaction of protein with the channel walls. 


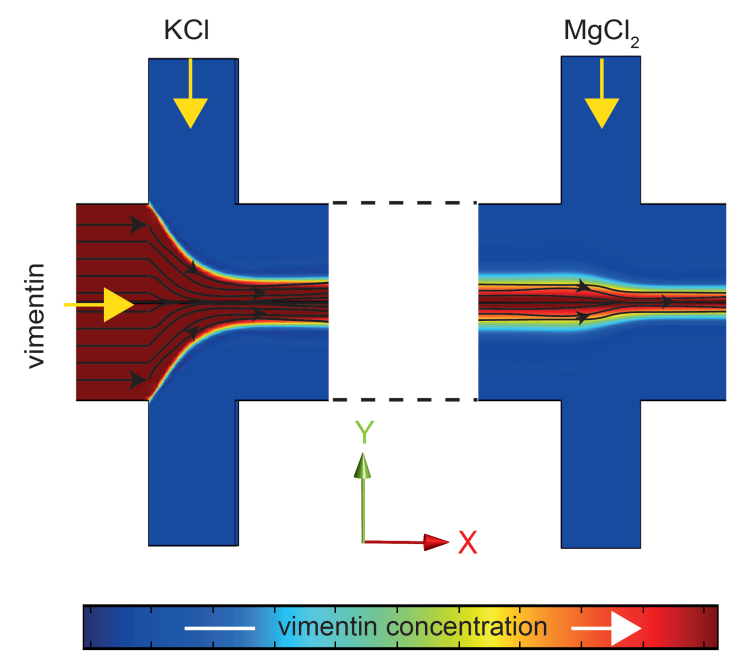

Figure 3.6.: Simulated hydrodynamic flow focusing in continuous flow device. The black lines correspond to the streamlines that are generated in the simulations based on the flow fields and flow rates. Figure taken from [63] with permission.

\subsubsection{Droplet-Based Microfluidics}

One of the major advantages of droplet microfluidics over continuous flow is the effective manipulation and control of discrete amounts of "capsules" of sample volume. Interest in droplet microfluidics has led to successful applications in solving problems related to protein fouling and aberrant crystallization, which are otherwise precarious in continuous flow. This leads us to use segmented flow as a tool to be able to handle the micro-reactions and consequently give us a good signal-to-noise ratio in the $\mathrm{X}$-ray experiments.

The microfluidic device geometry which is considered for the production of droplets is based on "T-junction" geometry where two or more components can be mixed [65, 66]. Production of droplets is explained in detail in the previously published work of Dammann et al. [52] where drop-manipulation (shape and frequency) is enabled solely by changing flow rates and geometry.

This setup contains three aqueous inlet channels that join up together in a sideby-side flow at low Re as shown in Fig. 3.7A and b. All three channels are maintained at the same flow rate to enable equal and homogeneous distribution of the the 3 components. In an alternate situation, one of the inlet channels can be increased (example: protein inlet) for a constant but higher volume, thus higher concentration compared to other components in the succeeding droplets. Once the 3-aqueous inlets join, the stream in flow focused by perpendicular flow of the 


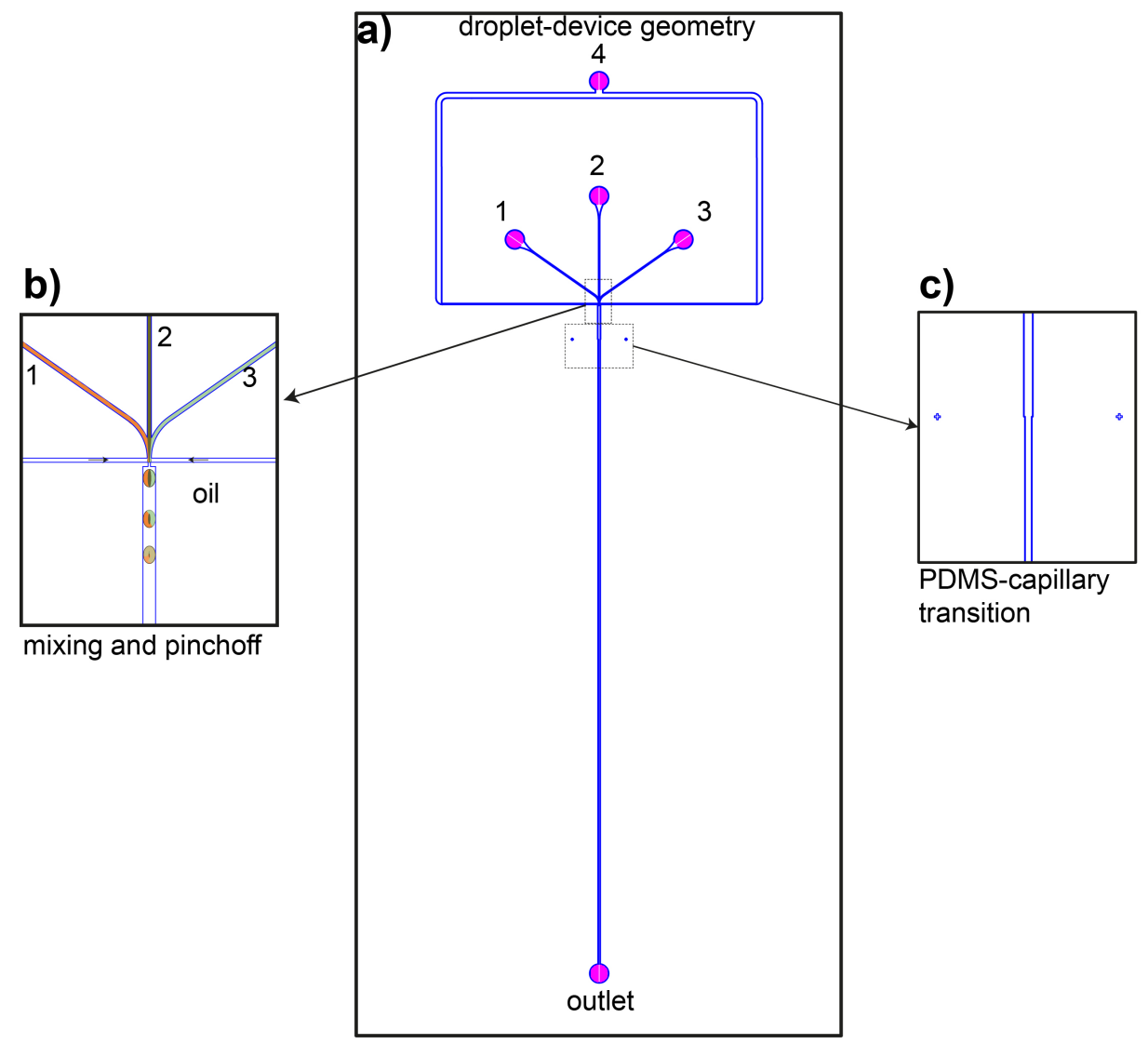

Figure 3.7.: Geometry of the channel used.a) A schematic diagram of the "T-junction" droplet geometry is shown b) zoom in of the droplet pinch-off region c) the transition from the droplet generation in PDMS to flow into quartz capillary is diagrammatically represented.

oil phase which leads to a "droplet pinch-off". These aqueous-in-oil droplets then flow in a straight channel and further into the quartz capillary for SAXS experiments (see Fig. 3.7k ).

\subsection{Device Implementation in Experiments}

\subsubsection{Continuous-Flow Devices in SAXS Experiments}

The major challenge involved in combining microfluidics with SAXS experiments is the usage of a microfluidic device which has a low X-ray absorption coefficient and high X-ray flux resistance. A channel which is impermeable to air or aqueous components (PDMS is avoided since fluids swell it up and flow profiles change 


\section{\begin{tabular}{l|l} 
Chapter 3 & MICROFLUIDICS FOR IN SITU INVESTIGATION OF BIO-PROCESSES
\end{tabular}}

thereby affecting evolving kinetics) is also preferred. To this end, a device made out of two layers of NOA-81 with a supporting PDMS layer was used, fabricated and adapted accordingly based on the idea of microfluidic stickers [67]).

In comparison to PDMS, NOA-81 has greater water resistance to swelling which makes it desirable for microflow experiments with aqueous components [68], as well as it ability of bio-compatibility [35]. This renders NOA-81 to be suitable for studies involving proteins and other bio-materials. Features of the micron-range are conveniently reproducible and the stickiness between the two-layered device can be controlled just with the exposure of the UV radiation. The adhesion property of this optical glue is excellent and does not require any heat, mechanical or chemical force for bonding as explained in Sec. 3.2.2.

During the fabrication of the device, the PDMS stamp is pressed into the NOA-81 to obtain an extremely thin layer of the design, which can then be cured. A thin layer is advantageous in SAXS experiments, especially, because the scattering signal is often weak in biological solution SAXS and reduction of parasitic scattering from the device itself is favorable. The transmission coefficient of cured NOA-81 is higher than PDMS. It also has an elastic modulus 3 orders of magnitude higher than PDMS (typically $1 \mathrm{GPa}$ ). This avoids sagging effects, even for very low aspect ratio shallow channels [67].

The geometry of the device is designed in such a way as to enable successive mixing of the ions into the centrally hydro-focussed jet as shown in Fig. 3.8. Results of this method of successive mixing in combination with tracking kinetic processes by SAXS are later discussed in Sec. 4.4.1.

\subsubsection{Droplet-Based Devices in SAXS Experiments}

One of the major advantages of using droplet based microfluidics over continuous flow device is to avoid the protein adherence (vimentin, in the present study) to microfluidic channel walls. Since our study dealt with the tracking of kinetic processes in microflow with SAXS, two challenges had to be realized with respect to device/technique usage:

- to fabricate a device compatible with droplet generation and

- to fabricate the device from a X-ray compatible material with low attenuation. 


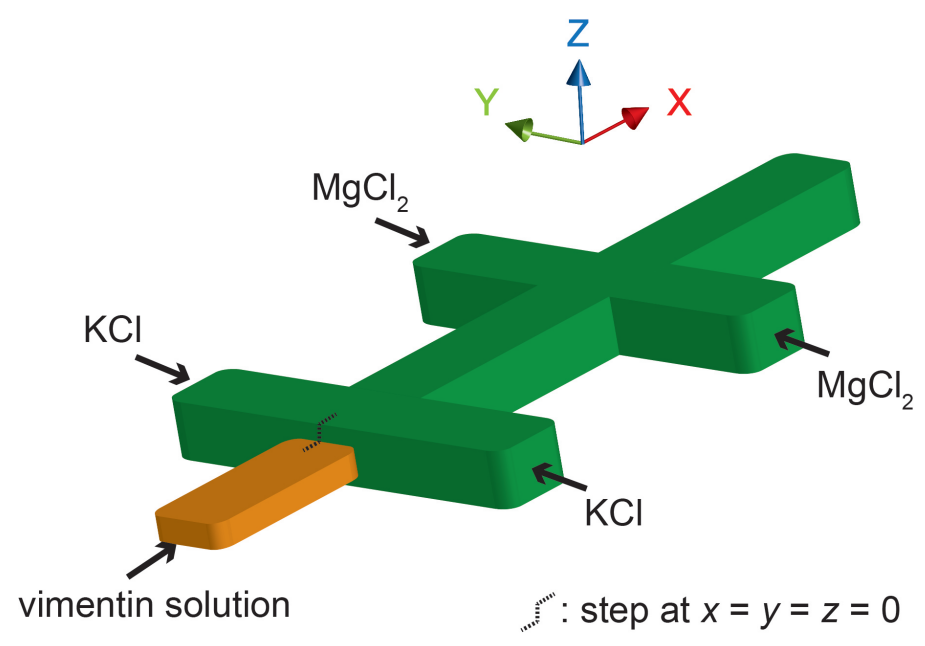

Figure 3.8.: Sketch of the continuous flow device geometry. The device geometry has one central inlet (orange, reduced height) and two sets of lateral inlets (for $\mathrm{KCl}$ and $\mathrm{MgCl}_{2}$, respectively). The step is incorporated such that the protein is engulfed by the buffer fluids to avoid protein adherence. Figure taken from [63] with permission.

As a solution to the above challenges, a composite PDMS-quartz capillary device is fabricated which is both droplet and X-ray compatible as shown in Fig. 3.9. The idea of the device is based on the work of Zheng et al., for performing protein crystallization trials in nanoliter aqueous droplets inside capillaries suitable for Xray diffraction and the evaluation of the quality of the crystals directly by on-chip $X$-ray diffraction [69]. We use the PDMS segment only to generate droplets that contain the protein, buffer and the salt ions by a procedure described in [52]. The segment with the quartz capillary is placed in the measurement window since the transmission coefficient is greater than PDMS and absorption is lower and the capillary can conveniently be used for two-phase experiments due to its water-inoil emulsion affinity (see Fig. 3.10

This procedure of the composite PDMS capillary has several advantages compared to the continuous flow device explained in Sec. 3.3.1. in addition to its droplet-X-ray compatibility such as: 1) It is robust in terms of withstanding flow rates as high as $700-1000 \mu \mathrm{l} / \mathrm{h}$ in a channel area cross-section of $1 \times 10^{4} \mu \mathrm{m}^{2} .2$ ) The flourinert oil (in the presence of a surfactant) used for generating droplets is non-destructible to both PDMS and the quartz capillary. Therefore, the composite PDMS-capillary device is a desirable tool for studies combining droplets and 


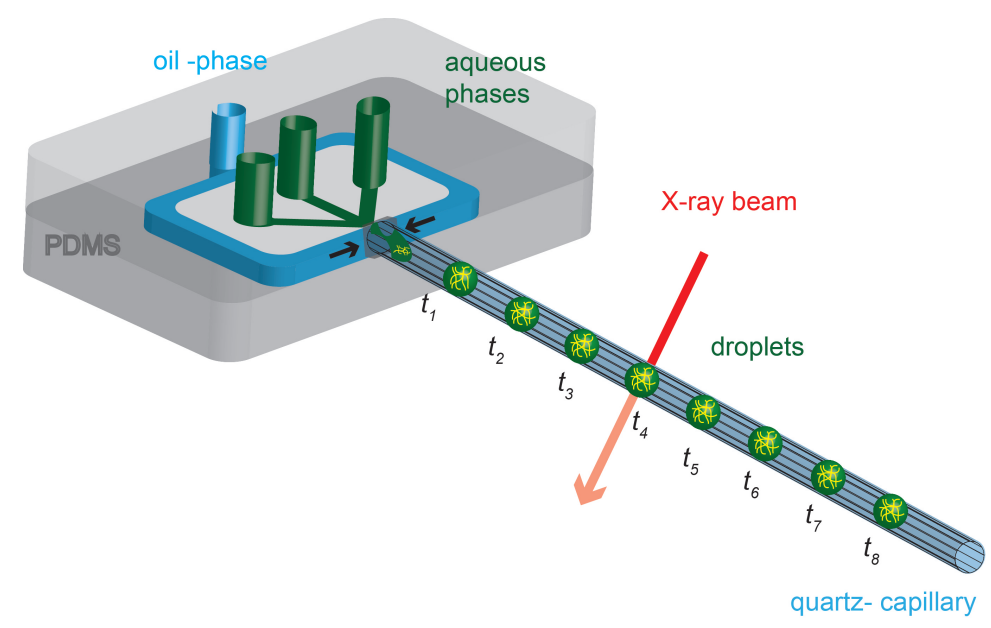

Figure 3.9.: Sketch of the droplet device. Oil and aqueous phases are shown in different colors to shown the generation of droplets. In the SAXS experiment, droplets are probed by the $\mathrm{X}$-ray beam in several locations

X-ray techniques due to its portability and endurance.

\subsection{FEM Simulations for Tracing Species-Distribution}

In order to understand the assembly kinetics for vimentin in flow, exact knowledge of the flow conditions, such as concentration distributions of salt and protein and velocities in each position of the device is essential. Therefore, we perform simulations of the laminar flow for the continuous flow device geometry as discussed in the previous sections. COMSOL multiphysics 4.4 and 5.0 (COMSOL $\mathrm{GmbH}$, Göttingen, Germany) is used to perform the finite element method (FEM) simulations of the flow conditions. COMSOL is a finite element solver and simulation software used to solve physics and engineering related problems. Here, FEM is employed, which is a numerical technique based on the approximation method to obtain solutions to the involved partial differential equations (PDEs) for a given geometry and boundary value problem. It is a mathematical approach in which a continuum problem can be solved by dividing the solution domain into smaller elements. These elements - finite elements - have the same properties of those who originated them but they are simpler to define and to reduce the number of unknowns. The solution of differential equations regarding the physical problem can be solved by approximated functions that satisfy the conditions described by integral equations in the problem domain. These approximated functions are usually polynomial functions [70].

Since our aim is to evaluate the flow conditions and the distribution of different 


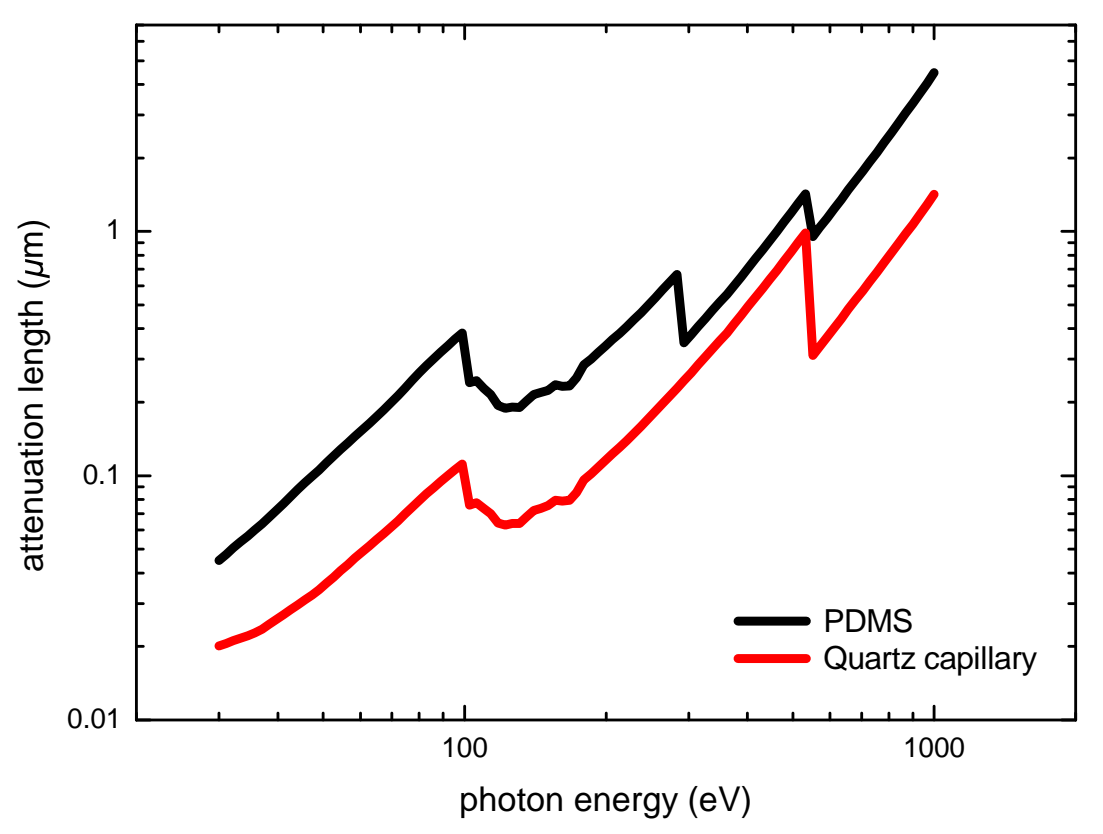

Figure 3.10.: Comparison of attenuation lengths. The data is generated by an online tool http://henke.lbl.gov/optical_constants/atten2.html for given chemical formula and densities

solute species in a microfluidic channel, we employ the Navier-Stokes equation (Eqn. 3.1) for the flow fields and the convection diffusion equation (Eqn. 3.4) for the solutes. Both equations need to be solved simultaneously to account for the interaction between the variables such as viscosity, local concentrations and distribution of the solutes.

Parts of this section (5.3.2 3.5.2 3.5.3) are published in the journal Biomicrofluidics [63] and is adapted here with permission. Author contributions are as follows: Oliva Saldanha performed the FEM simulations and analyzed the simulation results and correlated to the data analyzed from the X-ray experiments as discussed in the following chapters. Martha Brennich designed the microfluidic channels and performed the X-ray experiments. Oliva Saldanha has made the figures 3.113 .123 .14 for the manuscript and has contributed in iteratively writing it with Harald Hermann and Sarah Köster. 


\section{\begin{tabular}{l|l} 
Chapter 3 & MICROFLUIDICS FOR IN SITU INVESTIGATION OF BIO-PROCESSES
\end{tabular}}

\subsubsection{Model Geometry and Mesh}

In this work, we model a three-dimensional homogeneous geometry with the same material properties. The geometry consists of channel with a width of $300 \mu \mathrm{m}$ including buffer inlets and the outlet with a height of $240 \mu \mathrm{m}$. The central protein inlet has a height of $110 \mu \mathrm{m}$, which is centered with respect to the other channel. The total length of the device is modeled to be $10 \mathrm{~mm}$, taking into account from the start of the device. To reduce the computational effort and for symmetry reasons, only a quarter of the entire geometry is modeled (cut in the $x-z$ plane and the $y$ $-z$ plane) as shown in Fig. 3.11a. The lengths of the inlet channels are reduced in the model but are large enough to ensure the correct inflow and mixing profiles. The geometrical incorporation of the 'step' is zoomed in Fig. 3.11p. The step is reduced in dimension to avoid assembled vimentin to get in contact with the walls, which might lead to adhesion and eventually clogging of the channels during experiments [35, 71, 72, 73]. A maximum mesh size of $11.1 \mu \mathrm{m}$ and minimum mesh size of $0.724 \mu \mathrm{m}$ are used to define the tetrahedral mesh in the simulations. A zoom in of the mesh is depicted in Fig. 3.11k.

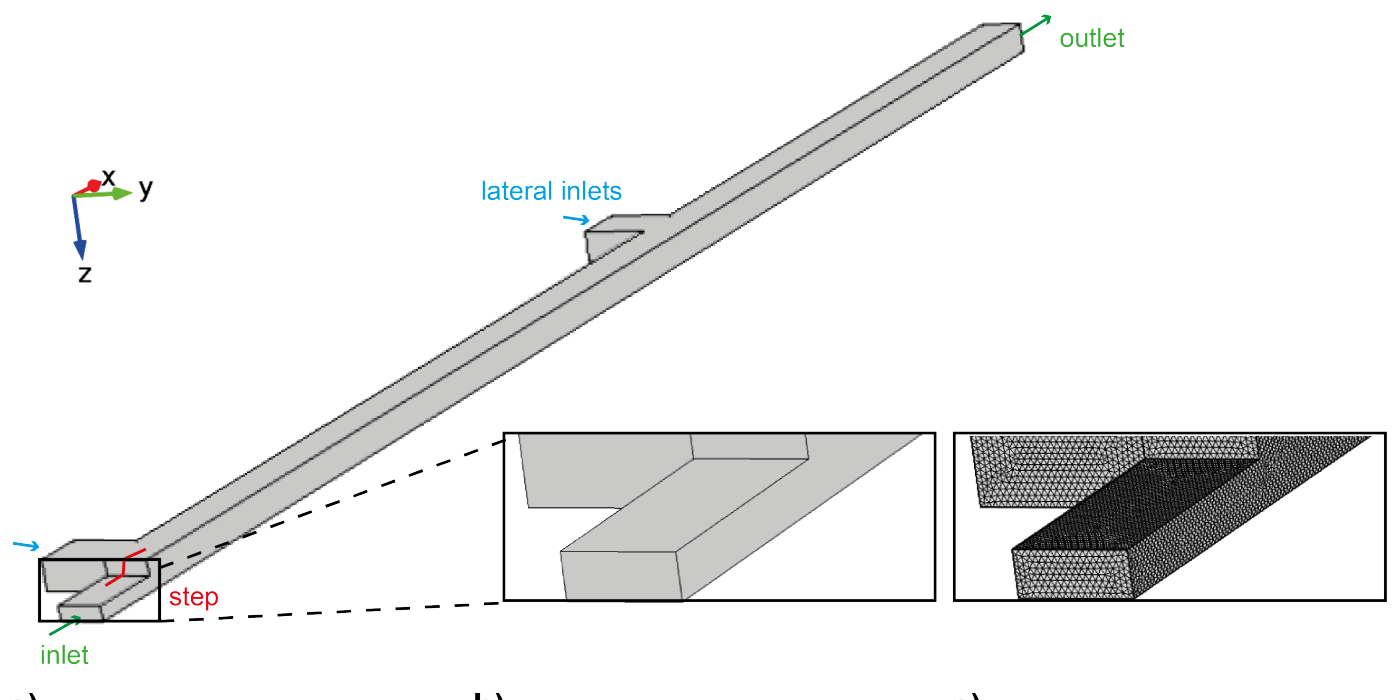

a)

b)

c)

Figure 3.11.: Geometry of the channel used for FEM simulations of continuous flow.a) Full length device for simulation. b) Zoom in of the step c) 3D mesh. 


\subsubsection{Global Constants and Varying Parameters}

Simulations are performed in the laminar flow regime and no-slip boundary conditions are used at channel walls. While running the simulation, the inflow velocity has a fixed constant value with the inlets long enough for the parabolic flow profile to develop. However, there are certain parameters such as the diffusion coefficients and the overall temperature, which are constant for the simulations whereas parameters such a flow speeds and concentrations vary for different conditions. The diffusion coefficients used in the simulations are listed in the Tab. 3.1. at temperature $293.15 \mathrm{~K}$ :

Table 3.1.: Diffusion coefficients used in the simulation

$$
\begin{array}{ll}
\text { vimentin [74] } & D_{\text {vim }}=2.4 \times 10^{-11} \mathrm{~m}^{2} \mathrm{~s}^{-1} \\
\mathrm{KCl}[75] & D_{K^{+}}=1.84 \times 10^{-9} \mathrm{~m}^{2} \mathrm{~s}^{-1} \\
\mathrm{MgCl}_{2}[76] & D_{\mathrm{Mg}^{2+}}=7.5 \times 10^{-10} \mathrm{~m}^{2} \mathrm{~s}^{-1}
\end{array}
$$

Since only a quarter of the entire device geometry is modeled, the flow velocity for the inlets are adapted accordingly. The central inlet takes into account only $1 / 4$ of the experimental flow rate, which remains constant when converted into flow velocity is $3.07 \times 10^{-4} \mathrm{~m} / \mathrm{s}$ which is the unit used in the simulation. Similarly, the flow velocity values considered for the lateral inlets are $1 / 2$ of the experimental values. The present simulation as shown in Fig. 3.12 is modeled with total flow rates of $40 \mu \mathrm{l} / \mathrm{h}$ in the central inlet and $80 \mu \mathrm{l} / \mathrm{h}$ in the lateral inlets and the results from other flow rates are mentioned in the Appendix. C.

\subsubsection{Representative Results from Simulations}

As explained earlier, these simulations are performed in congruence with understanding the behavior and evolution of vimentin in the presence of mono and di-valent salts during experiments. In this section, we intend to assimilate knowledge about the change in concentration of the respective species and results from generating streamlines along the central jet to explain time evolution of the reaction kinetics. 


\section{\begin{tabular}{l|l} 
Chapter 3 & MICROFLUIDICS FOR IN SITU INVESTIGATION OF BIO-PROCESSES
\end{tabular}}

Flow-Focusing

Vimentin is injected into the central inlet and hydrodynamically focused by the first lateral inlets ( $\mathrm{KCl}$ inflow). At $5.5 \mathrm{~mm}$ downstream, it is focused again by the second set of lateral inlets $\left(\mathrm{MgCl}_{2}\right.$ inflow) (see the cut in the $x-y$ plane in Fig. 3.12 a,b,c,d). The focused flow achieved in one quarter of the device geometry is sufficient to mimic the actual three-dimensional flow. Therefore, the values obtained along the streamlines of this simulation are valid and the results obtained due to the flow focusing are theoretically reproducible [63].

\section{Change in Concentration of Different Species}

Due to the small diffusion coefficient of vimentin, the concentration of vimentin decreases merely by a factor of 2 from $4 \mathrm{mg} / \mathrm{mL}$ at the inlet to $2 \mathrm{mg} / \mathrm{mL} 10 \mathrm{~mm}$ downstream at the last measurement position and the protein remains mostly in the center of the laminar flow. By contrast, the much smaller and therefore faster diffusing ions move into the central stream more readily and the final concentration of $70 \mathrm{mM} \mathrm{KCl}$ becomes distributed evenly across the channel width at about $4 \mathrm{~mm}$ downstream from the step at the central inlet. From here onwards, the $\mathrm{KCl}$ concentration remains the same throughout the channel length (Fig. 3.12p). The concentration of $\mathrm{MgCl}_{2}$ increases approximately linearly from zero to $\approx 4.5 \mathrm{mM}$ (Fig. 3.12 c) starting from the second set of lateral inlets. The line-graph of the change in the salt concentrations is shown in Fig. 3.13 [63].

\section{Calculation of Shear Stresses}

There is an overall increase in the velocity magnitude over the length of the channel (Fig. $3.12 \mathrm{~d}$ ) due to the additional inflows from the second set of lateral inlets. The increase in the velocity magnitude can be utilized to estimate the average shear rates in the channel. Estimating the shear rates:

$$
\dot{\gamma}_{x y}=\left(\frac{\partial v_{x}}{\partial x_{y}}+\frac{\partial v_{y}}{\partial x_{x}}\right)
$$

we arrive at $\approx 3.2 \mathrm{~s}^{-1}$ between the first and second set of lateral inlets and $\approx 5.5 \mathrm{~s}^{-1}$ from the second set of lateral inlets onwards. In order to derive the shear stress from these shear rates, the viscosity of the fluids needs to be taken into account. Since we consider only the first few seconds of assembly, and in agreement with viscosity measurements form literature [77], we assume water viscosity and 
a)

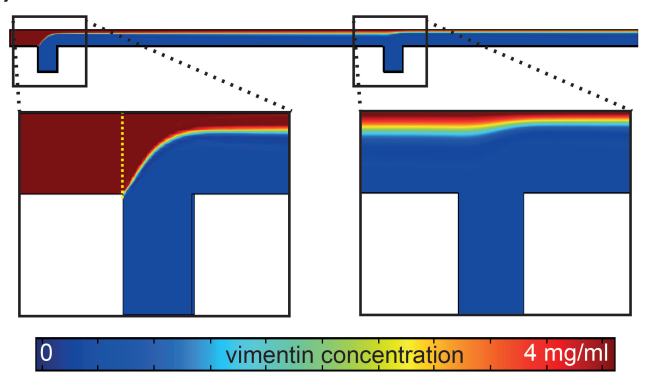

c)

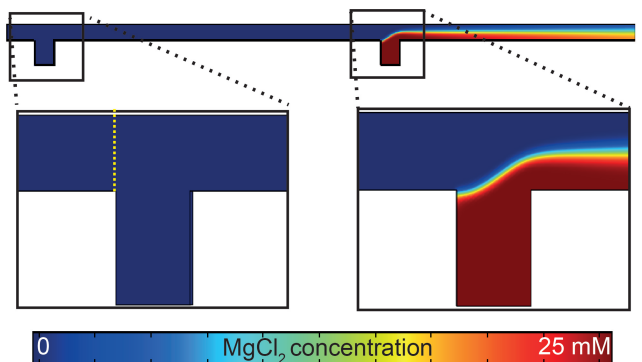

b)

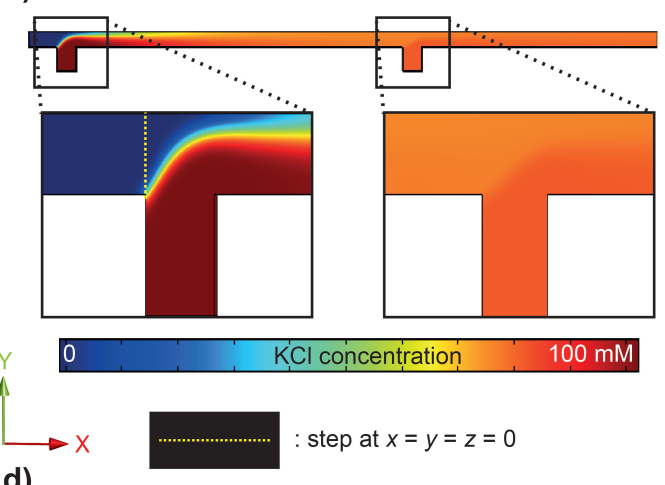

d)

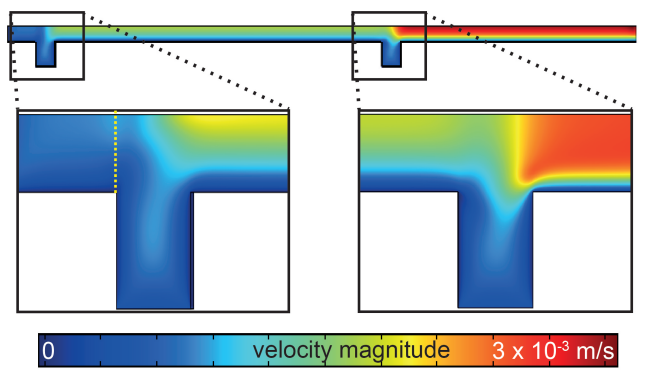

Figure 3.12.: FEM simulations of the flow conditions and local concentrations in the microfluidic devices. Simulations shown here are for a vimentin flow speed (central inlet) of $335 \mu \mathrm{m} / \mathrm{s}\left(40 \mu \mathrm{L} \mathrm{h}^{-1}\right)$ and salt buffer flow speeds for each lateral inlet of $310 \mu \mathrm{m} / \mathrm{s}\left(80 \mu \mathrm{L} \mathrm{h}^{-1}\right)$. The mid-plane $(x-y)$ is shown. (a) Vimentin concentration in the device. Enlarged are parts of the inflow of $\mathrm{KCl}$ (first cross) and $\mathrm{MgCl}_{2}$ (second cross). The vimentin concentration remains high in the central part since the large proteins diffuse slowly; the concentration drops only from $4 \mathrm{mg} / \mathrm{ml}$ to $2 \mathrm{mg} / \mathrm{ml}$ during the entire length of $10 \mathrm{~mm}$ (b) Change in $\mathrm{KCl}$ concentration. $\mathrm{KCl}$ is injected at the first cross, diffuses into the central protein stream and remains at a constant concentration when $\mathrm{MgCl}_{2}$ is injected. (c) Change in $\mathrm{MgCl}_{2}$ concentration. (d) Velocity magnitude. Figure taken from [63] with permission.

thus derive shear stresses on the order of $5 \mathrm{mPa}$. We did not include the change in viscosity upon assembly in our simulation since viscometry measurements show that the viscosity increases only very little during the first seconds of the assembly process [34, 39]. 
Figure 3.13:

Results derived from the FEM simulations

shown in Fig.

3.12 for the central flow line $(y=z=0)$ where the concentrations of $\mathrm{KCl}$ and $\mathrm{MgCl}_{2}$ are shown. Figure taken from [63] with permission.

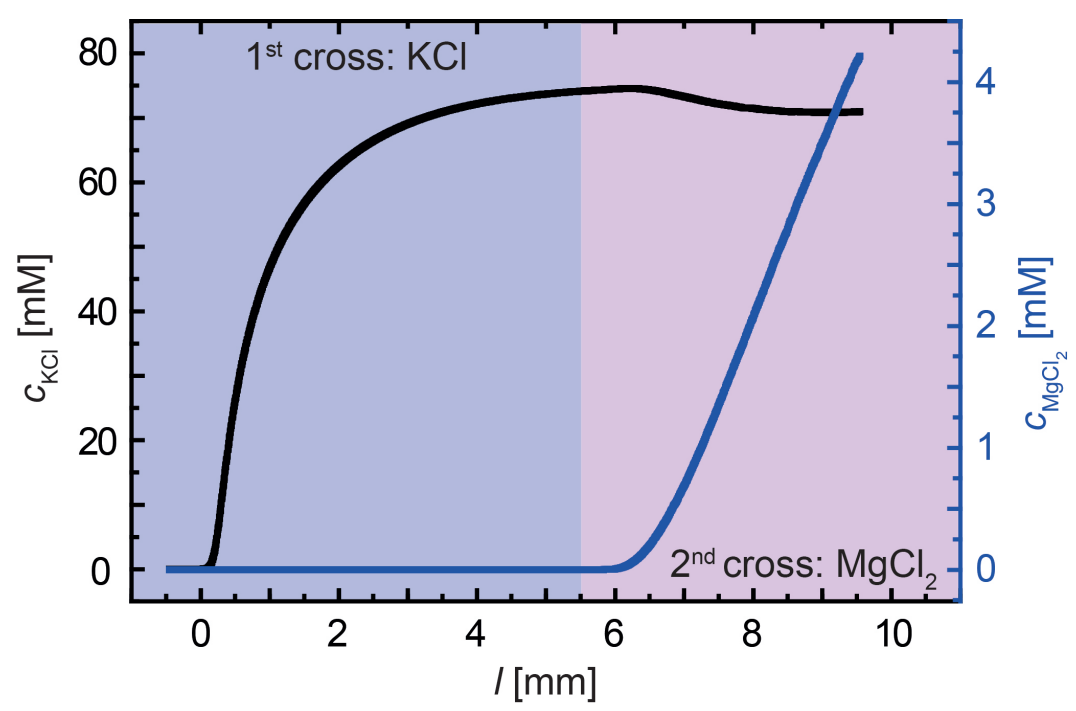

Determination of Time Evolution for Reaction Kinetics in Microflow

Despite the obvious advantage of microfluidic mixing techniques employed to ensure fast reaction kinetics, the discrete time scale calculation is not straight forward. This is due to the absence of a well-defined zero time point and convolution of mixing and reaction kinetics in flow. For determining the time evolution of the involved kinetics, we employ the LiveLink for MATLAB provided by COMSOL to first identify the streamlines which are crucial for a region of interest and then integrate over a particular streamline to obtain the reaction times in an adapted MATLAB code described in appendix A, based on the doctoral thesis of Martha Brennich [50]. The MATLAB LiveLink combines the powerful FEM abilities of COMSOL with the versatile programming environment of MATLAB. In order to investigate the time scales on which the assembly reaction takes place, streamlines in the flow are calculated starting at $x=-0.35 \mathrm{~mm}$ with a spacing $\Delta y=1 \mu \mathrm{m}$ between the streamlines at the position of the inlet, but for better visibility only a few streamlines are shown in Fig. 3.6. However, volume elements of protein solution are tracked only along the central streamline starting at $z=y=0$. The concentration of vimentin along this streamline is assumed to be constant because $D_{\text {vim }}$ is rather small. The involved time scales are derived by integrating over the inverse of the flow velocity along a streamline [63]:

$$
t(s)=\int_{0}^{s} \frac{1}{v(s)} \mathrm{d} s .
$$




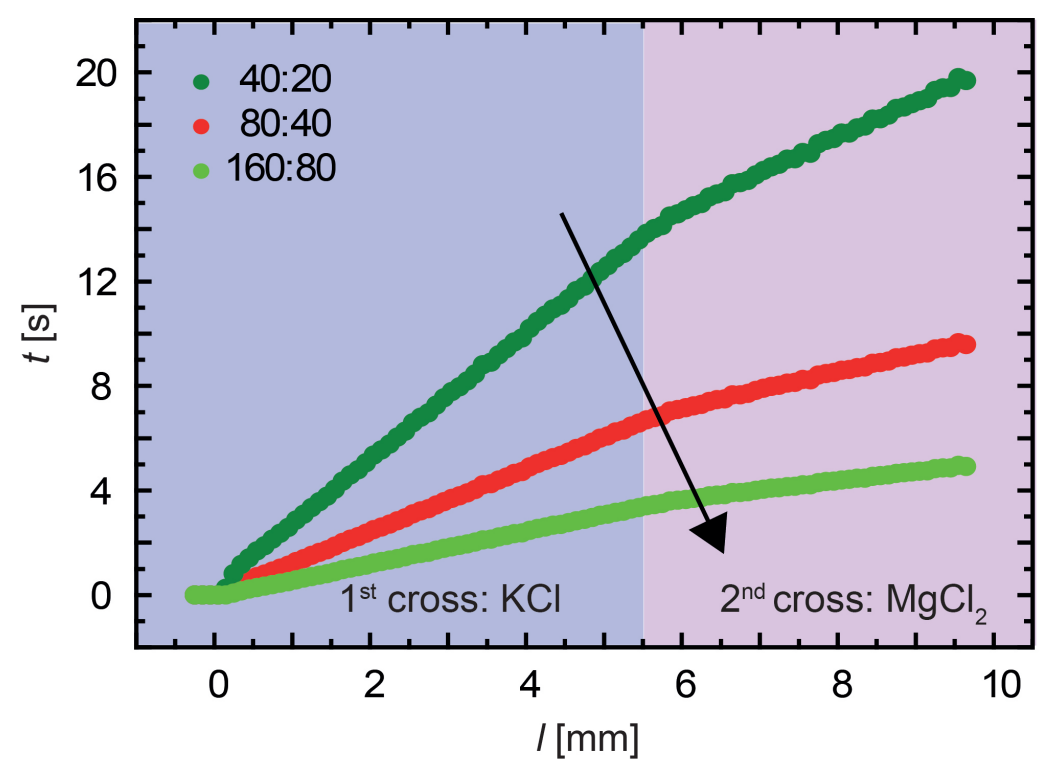

Figure 3.14:

Time evolution along the channel for different flow rates. The black arrow indicates increasing flow rates. The legend shows the respective flow speed: first number - lateral buffer inlets; second number central vimentin inlet. Figure taken from 63. with permission.

The results for the different sets of flow rates used in the experiments are displayed in Fig. 3.14 . For the example of the simulation shown in Fig. $3.12\left(40 \mu \mathrm{L} \mathrm{h}^{-1}\right.$ in the central inlet and $80 \mu \mathrm{L} \mathrm{h}^{-1}$ in the lateral inlets) the flow time from the step to the second set of lateral inlets is $7 \mathrm{~s}$ and the total flow time is $9.5 \mathrm{~s}$ (red curve). For reduced flow rates (dark green curve), the total time is $19.8 \mathrm{~s}$ and for faster flow rates [63]. 



\title{
Small Angle X-ray Scattering for Protein Assembly Kinetics
}

\author{
"Broadly speaking, the discovery of \\ $X$-rays has increased the keenness \\ of our vision ten thousand times, \\ and we can now 'see' the \\ individual atoms and molecules..."
}

William Henry Bragg, 1925

Small angle X-ray Scattering (SAXS) is a fundamental technique to elucidate the structural information of matter in general and biological molecules in particular [55]. When radiation interacts with inhomogeneities in a given macromolecular sample, there is a deviation from the primary incident intensity resulting in static elastic scattering at small angles. These recorded scattered intensities contain information of the structure of the native particles in near-physiological conditions. This is an advantage because the SAXS tool offers access to the biomolecular structure close to their natural functioning state without the need to crystallize. SAXS, not only considers the basic biological configurations but also offers an opportu- 
nity to analyze the changes that might occur due to the effect of external conditions like change in concentration, $\mathrm{pH}$ and the effect of ions. Though this technique has been traditionally perceived to be a low resolution tool (due to feeble signal obtained from biological samples) in comparison to NMR and X-ray crystallography, what makes it a powerful tool is the ability to distinguish between possible conformations in solution due to the direct and precise derivations from the atomic scale, which is much closer to physiological conditions.

The information obtained and the analysis methods used, differ from conventional crystallography. In a crystalline material, the diffraction pattern following the Bragg's law, has a set of sharp peaks whose positions are given by the atomic spacing in the material. However, in solution SAXS, we do not obtain any characteristic peak but a smooth varying intensity profile at the relevant scattering angles. The signal obtained is characteristic of the the size which arises due to the inhomogeneities that occur in a sample and the larger the size of the particle, the lower is the scattering angle and vice versa [55]. When the scattering angles are detected on a plane, it is always considered to be the interference of all scattering waves due to their respective electron densities over all orientations.

This chapter focuses on SAXS as a powerful tool for the investigation of macromolecules where a few essential basic concepts are introduced in Sec. 4.1. A brief explanation of the different experimental setups from which the data is discussed in this thesis is presented in Sec. 4.3. The data analysis employed and the relevant results from the two different experimental approaches of continuous and droplet-based microfluidics are presented, respectively, in Sec .4.4.1 and 4.5.

\subsection{General Theory}

In a basic scattering experiment, when $\mathrm{X}$-rays irradiate a sample, two (elastic and inelastic) of the many kinds of interactions (other interactions that occur are photo disintegration, pair production etc) that occur can be considered:

- the electrons within the atoms of the sample are excited which resonate the same frequency as the incident radiation and scatter coherent secondary waves in all directions. In the forward direction all of them scatter in phase, resulting in maximum intensity and the background radiation is almost constant at small angles. The number of photons scattered is determined as a function of scattering angle $2 \theta$. 
- as we move to larger $2 \theta$, there is some degree of destructive interference occurring due to the particles (i.e., clusters of atoms) within the sample which corresponds to a decreasing intensity. If the radius of the particle is $R$, then the small angle scattering occurs for particles whose size is very much larger than the wavelength $\lambda$.

Typically, length scales probed with SAXS are in the 1-100 $\mathrm{nm}$ range, using X-rays of wavelength of the order of $0.1 \mathrm{~nm}$. Therefore, small angle X-ray scattering is produced by inhomogeneities in the electron density of a material at length scales much larger than the X-ray wavelength.

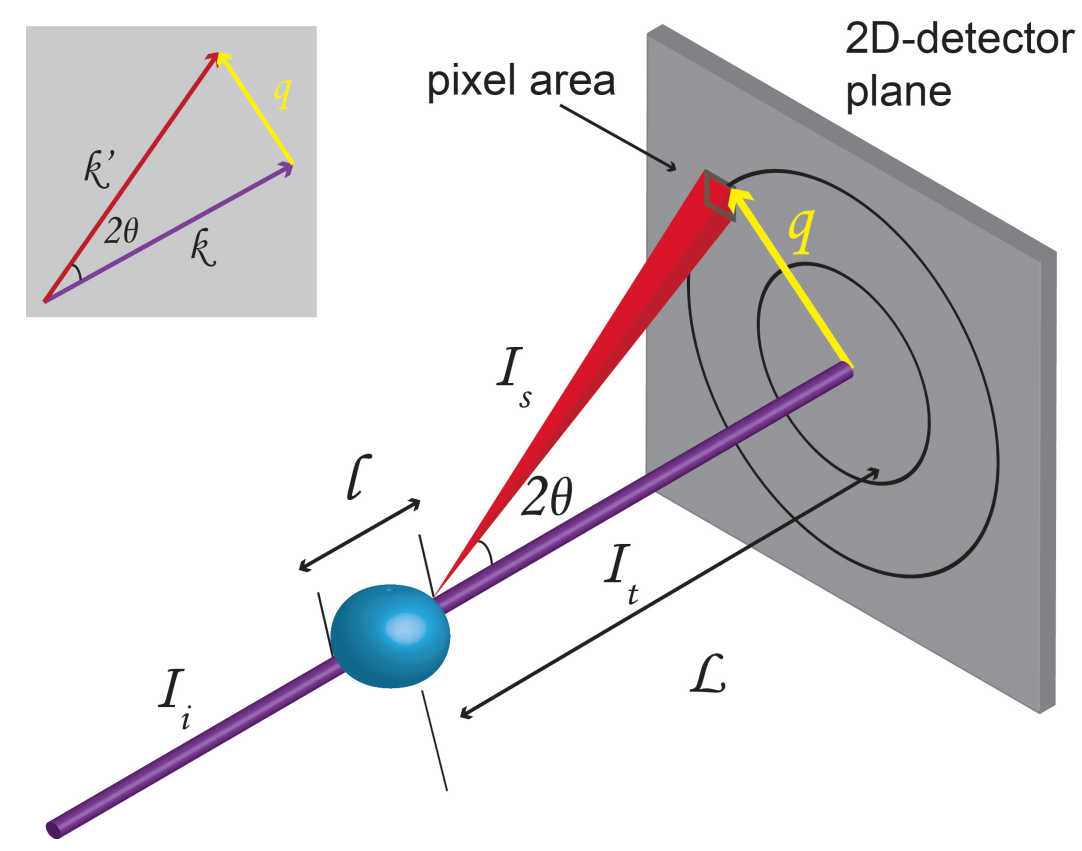

Figure 4.1.: Schematic $\mathbf{X}$-ray scattering diagram. The inset shows a vector diagram of the elastic scattering process; $\vec{k}$ and $\vec{k}^{\prime}$ are the reciprocal vectors of the incoming and scattered beams, $\vec{q}$ is the scattering vector.

The basic scattering process is shown in the Fig. 4.1. $I_{i}$ is the incident intensity, $I_{t}$ is the transmitted intensity and $I_{S}$ is the scattered intensity. $l$ and $L$ are the lengths of the sample and the sample distance from the detector, respectively. Also, $\vec{k}$ and $\overrightarrow{k^{\prime}}$ are wavevectors of incident and scattered waves respectively. The total momentum transfer in the sample is defined as the "scattering vector" $\vec{q}$, where $\vec{q}=\vec{k}^{\prime}-\vec{k}$. Since the scattering, in the present case, is elastic $|\vec{k}|=\left|\overrightarrow{k^{\prime}}\right|$, the magnitude of $\vec{q}$ in terms of the scattering angle $2 \theta$ is given by: 


$$
q=\frac{4 \pi}{\lambda} \sin \theta
$$

where the dimension of $q$ is one over length (i.e., $\left[\frac{1}{n m}\right]$ ), and the scattering pattern is usually called the "structure in reciprocal space" whereas the particles within the given sample are said to have "structure in real space" whose dimensions are in $[\mathrm{nm}]$. Hence, the interference patterns obtained on the given detector plane are characteristic of the internal structure of the material based on the orientation and distances of the atoms relative to each other.

\subsubsection{Form Factor and Structure Factor}

Let us consider a system of $n$ electrons which have a charge density distribution of $\rho(\vec{r})$, where $\vec{r}$ is the spatial variable. The electromagnetic field around the object can be identified as either far-field (i.e., the relationship between electric field component $\mathrm{E}$ and magnetic field component $\mathrm{H}$ is characteristic of any freely propagating wave, where (in units where $c=1$ ) $\mathrm{E}$ and $\mathrm{H}$ have equal magnitudes at any point in space) or near-field (i.e., the diffraction pattern in the near field differs significantly at infinity and varies with distance from the source and the relationship between $\mathrm{E}$ and $\mathrm{H}$ becomes very complex). The far-field scattering approximation can be applied in this context if the sample system size is less than the sample-to-detector distance. An X-ray plane wave $\vec{k}_{i}$ with an electric field strength of modulus $\vec{E}_{i}$ is incident on the electrons in the system exciting them which in turn results in the emission of secondary waves. For a single scattering event, the wave amplitude is $E_{f}(\vec{r})$ and the secondary wave undergoes further scattering only when the interaction between the electronic system and the electromagnetic field is stronger. The resulting wave amplitude can be denoted in the kinematic approximation where the incident wave is hardly modified by the material as it passes through. Therefore, the scattered wave is seen as a small perturbation due to an interaction of a very weak magnitude similar to the Born approximation [78] (which is also a perturbation method applied to a scattered body where the perturbation is considered accurate if the scattered field is small compared to the incident field), as:

$$
E_{f}\left(\overrightarrow{r^{\prime}}\right)=E_{i} \cdot e^{i \vec{k}_{i} \cdot \vec{r}^{\prime}}+E_{i} \cdot \frac{e^{i k_{i} \cdot r^{\prime}}}{r^{\prime}} \cdot r_{0} \cdot \int_{V o l} \rho(\vec{r}) e^{i \vec{q} \cdot \vec{r}} d^{3} r
$$


The first term on the right hand side in Eqn. 4.2 is the non-interacting primary plane wave which is normally blocked by the beamstop in an experiment. The second term in Eqn. 4.2 is the scattering contribution.

Now, in order to arrive at the scattering intensities as a function of $q$ from Eqn. 4.1. we consider the following: spherical waves interfere and are detected at a distance $L$ (typical at the detector plane) and the origin of these spherical waves are from an infinitesimal element $\rho(\vec{r}) d^{3} r$ in the given sample system. The amplitude of the so-called scattered wave is mathematically proportional to the $\rho(\vec{r})[79]$ and is defined as:

$$
A(\vec{q})=r_{0} \cdot \int_{V o l} \rho(\vec{r}) e^{i \vec{q} \cdot \vec{r}} d^{3} r
$$

where $r_{0}$ is the Thomson scattering length (which is the classical electron radius based on the relativistic model of the electron) and the product of $r_{0}$ and $\rho(\vec{r})$ is known as the "scattering length density" which is a measure of the scattering power of a material. It is crucial in determining the contrast as explained in detail in Sec. 4.1.2.

Knowledge of $A(\vec{q})$ (a complex function) from Eqn. 4.3 is sufficient to determine the structure of a particle by obtaining the inverse of $\rho(\vec{r})$ of that particle. However, the scattering intensity of one single particle $I_{0}(\vec{q})$ is given by the product of the amplitude $A(\vec{q})$ and its complex conjugate $A(\vec{q})^{*}$ where:

$$
I_{0}(\vec{q})=A(\vec{q}) \cdot A(\vec{q})^{*}
$$

The scattering amplitude is not accessible by experiment but only the magnitude of $A$ can be obtained which is equal to the intensity of the scattered wave and for a symmetric particle

$$
I_{0}(\vec{q})=|A(\vec{q})|^{2}=(\Delta \rho)^{2} V_{p}^{2} P(\vec{q})
$$

where $\Delta \rho$ is the difference in electron densities and is dealt with detail in Sec. 4.1.2, $V_{p}$ is the volume of the particle and $P(\vec{q})$ is the form factor of a single particle which determines the shape and size of that particle.

However, for a system of dilute monodisperse particles which are non-interacting, the total scattered intensity $I(\vec{q})$ of particles is then the sum of the intensities scattered by each particle and is given by:

$$
I(\vec{q})=\sum_{i} I_{0}(\vec{q}, i)=\sum_{i}(\Delta \rho)^{2} V_{p, i}^{2} P(\vec{q})
$$




\section{\begin{tabular}{l|l} 
Chapter 4 & SMALL ANGLE X-RAY SCATTERING FOR PROTEIN ASSEMBLY KINETICS
\end{tabular}}

For a system of inter-particle interactions, an interference term which is called the structure factor $S(\vec{q})$ has to be introduced:

$$
I(\vec{q})=\sum_{i}(\Delta \rho)^{2} V_{p, i}^{2} P(\vec{q}) S(\vec{q})
$$

The structure factor $S(\vec{q})$ bears information of the particle-particle interactions such as inter-particle distance and the degree of order. In the analysis performed for the data presented in this thesis, $S(\vec{q})$ is assumed to have a value of 1 due to geometrical constraints that determine the $q$-range.

\subsubsection{Concept of Contrast in Particle Solutions}

A crucial feature that plays an important role in X-ray scattering is the 'contrast' between the solute and the solvent. The 'contrast' or the 'change in electrondensity' has a strong influence on biological molecules which are in their native form synonymous with inhomogeneous internal density distribution. Therefore, it is essential to choose a solvent which provides an appropriate contrast.

Since the scattering intensity of a monodisperse system such as Eqn. 4.6 (for the sake of simplicity) is dependent on its electron density $\overrightarrow{\rho(r)}$, we get a spatially varying function comparable to inter-atomic separations in the system. If these particles are dispersed in a pure solvent with electron density of $\rho_{s}$, then for scattering at small angles, the scattering density depends on the difference between the mean particle electron density and the solvent electron density:

$$
\Delta \rho=\langle\rho(\vec{r})\rangle-\rho_{s}
$$

as only the contrast between a particle and solvent can lead to the non-zero scattering intensity in Eqn 4.6. For high values of $\Delta \rho$ where $\Delta \rho \geqslant \rho_{s}$, contrast is expected to be strong where the internal shape and structure can be determined clearly against the solvent background. On the other hand, at low contrast for $\Delta \rho \approx 0$ or $\rho \overrightarrow{(r)} \approx \rho_{s}, \Delta \rho$ is determined by the particle inhomogeneities such that the particles in solution appear smeared and scattering intensities are close to zero [80]. Therefore, contrast is an important sample parameter in SAXS. Changing the contrast of the solvent density increases the possibility to obtain additional structural information such as in small angle neutron scattering (SANS) where the particles are deuterated routinely in solution [81]. 


\subsection{Methodology of Data Treatment}

In case of solution scattering, the 2D-scattering patterns acquired on the detector plane during the experiment are further subjected to a thorough data reduction either by radial or azimuthal integration to obtain the 1D-information. Secondly, these 1D-curves are extended to elaborate model-free data fitting methods such as Guinier approximation [82] and Porod's analysis [83] in the respective wavelength limits. This methodology is extended to elucidate the relevant physical parameters like the form factor (i.e., size, shape, surface) and the structure factor (i.e., length scales of inter-atomic interactions) of the system in question as discussed in Sec. 4.1. In this section, we discuss the relevant resolution limits necessary and a brief explanation of the model-free analysis techniques employed for the data presented in this thesis.

\subsubsection{Resolution Limit}

SAXS is a sensitive technique whose results can elucidate structural details of a system i.e., size, shape and surface. However, the resolution limits are crucial. Fixed geometrical limitations of the experimental setup such as the sample-todetector distance determine the observation window of the sample and hence establish the resolution scale. Theoretically, measurement at a given $q_{\text {ideal }}$ allows the observation of density fluctuations in a sample at a distance of $D_{\text {ideal }}=\frac{2 \pi}{q_{\text {ideal }}}$. This distance is equivalent to an "observance" window of diameter $\frac{2 \pi}{q_{\text {ideal }}}$ in real space. This concept of the required resolution is illustrated in an example as shown in Fig. 4.2. Consider an ensemble of randomly oriented homogeneous particles,

- the observance window "red" gives us information of the low q-domain in real space where the window is large and accommodates several particles within. The structural order of these particles can be elucidated which allows the interactions in the system.

- the observance window "green" determines the size, shape and internal structure of one particle and can be termed as the intermediary $q$-domain.

- the window "yellow" is very small and the contrast is only seen at the interface between the two media. This high $q$-domain called the Porod's region gives us information of the surface and interfaces. 


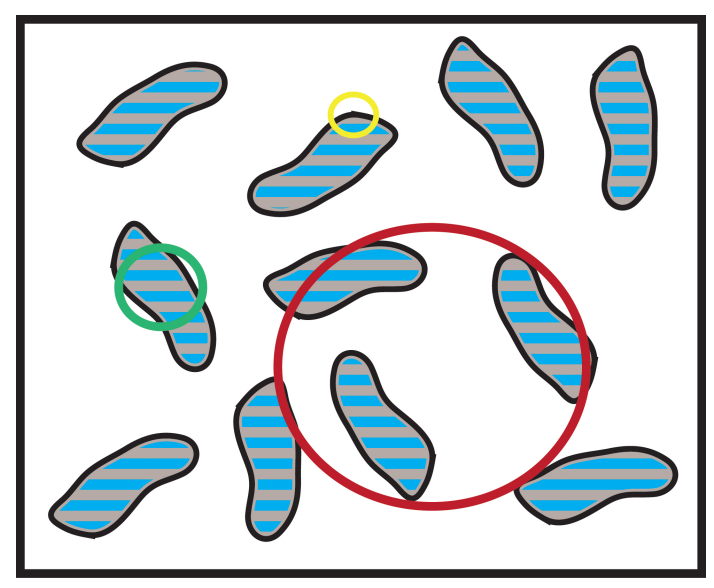

Figure 4.2.: Simple representative sketch to understand the resolution limits. Each color determines the window of observance such as red gives us the structure factor, green gives us the form factor and yellow gives us information about the surface and interfaces. Figure is based on the tutorial 84 .

With the above considerations, data measured in the relevant resolution limit can now be subjected to further morphological analysis which will be discussed in the following sections.

\subsubsection{Guinier Approximation}

Guinier approximation can be applied to scattering at low angles which gives us information about the intermediary $q$-domain from the explanation in Sec. 4.2.1 and Porod analysis can be applied for extracting the information at higher scattering angles. These model-free analysis tools are based on information at hand and not any previously known sample structure [83].

In the long wavelength limit (i.e., the long wavelength limit is the limiting case when the wavelength is much larger than the system size and is not applicable to the value of the $X$-ray wavelength that we include in our experiments) where $q R \rightarrow 0$, a Gaussian curve can be approximated at small angles which gives us information of the relevant form factor $P(\vec{q})$ and was first derived in 1939 by André Guinier. The approximation is based on the linearization of the data at small angles close to the zero scattering angle and the curvature of the Gaussian at these small scattering angles is due to the overall size of the particles in a system. Therefore, the overall intensity of a system of particles as described by Debye (1915) for 
spherical particle geometry can be written as,

$$
I(q)=\int_{0}^{4 \pi} p(r) \frac{\sin (q r)}{q r} d r
$$

where $p(r)$ is the particle distance distribution function (i.e., distribution of distances between pairs of particles contained within a given volume) and $I(q)$ is the Fourier transform of the $p(r)$ function. By expanding the sinusoidal function in the scattering intensity in a series, we get,

$$
\left[\frac{\sin (q r)}{q r}=1-\frac{q^{2} r^{2}}{6}+\frac{q^{4} r^{4}}{120}-\ldots\right]
$$

and now the Debye-function i.e., Eqn. 4.9 can be expressed as:

$$
I(q) \cong \int_{0}^{4 \pi} p(r)\left(1-\frac{q^{2} r^{2}}{6}+\ldots\right)=\int_{0}^{\infty} p(r) d r-\frac{q^{2}}{6} \int_{0}^{\infty} p(r) r^{2} d r+\ldots
$$

The second moment of the distance distribution function is given by:

$$
\int_{0}^{\infty} p(r) r^{2} d r
$$

and the radius of gyration $R_{g}$ can be introduced in analogy with classical mechanics as:

$$
R_{g}^{2}=1 / 2 \frac{\int_{0}^{4 \pi} p(r) r^{2} d r}{\int_{0}^{4 \pi} p(r) d r}
$$

Therefore by substitution in Eqn. 4.11. $I(q) \cong \int_{0}^{4 \pi} p(r)\left(1-\frac{R_{g}^{2}}{6} q^{2}+\ldots\right) d r$

The first term in Eqn 4.14 i.e., $\int_{0}^{4 \pi} p(r) d r$ becomes $I_{0}$ and by substituting, Eqn. 4.14 becomes:

$$
I(q) \cong I_{0}\left(1-\frac{q^{2} R_{g}^{2}}{3}\right)
$$

Eqn. 4.15 is not a linear equation and the term $1-\frac{q^{2} R_{g}^{2}}{3}$ can be expanded in the Taylor series of $e^{x}$. This substitution is an approximation and requires the $q$ to be small. Therefore, from Taylor series expansion of $e^{x}$ is given to be:

$$
e^{x}=1+x+\frac{x^{2}}{2 !}+\frac{x^{3}}{3 !}+\ldots \text { where } x=\frac{q^{2} R_{g}^{2}}{3}
$$


By substituting Eqn. 4.16 in Eqn. 4.15, we get the Guinier approximation for spherical particles to be:

$$
I(q) \cong I_{0} e^{-\frac{q^{2} R_{g}^{2}}{3}}
$$

where $R_{g}=\sqrt{\frac{3}{5}} R$ which gives the overall size of the particle and $R$ is the radius of the spherical particle. However, the guinier regime for spherical particles is only accessible upto $q R_{g}<1.3$. Generally, SAXS studies of protein kinetics interpret structural changes due to scattering from smaller angles. This interpretation is based on the Guinier approximation which gives the average radius of gyration predicted to follow a relationship of the form for long rods:

$$
I(q) q=I_{0} e^{\frac{-q^{2} R_{c}{ }^{2}}{2}}
$$

where $R_{c}{ }^{2}$ is the radius of gyration of cross section of a rod and $I_{0}$ is the extrapolated zero-angle intensity. However, scattering from larger angles also contains valuable information and is described in detail in Sec. 4.2.3.

\subsubsection{Porod's Law}

The scattering at higher angles of a given $q$-range, essentially characterizes the interface or surface between two media. This approximation in SAXS analysis is called the Porod-Debye law and was first proposed by Günter Porod in 1951 [83]. According to this law, the scattered intensities at a small wavelength $(q R \gg 1$ with the characteristic particle size $R$, but with the $q$ value small enough to not being sensitive to the atomic spacing [85]) follows a asymptotic decay which allows to calculate the surface $S$ of the particles.

When $q R \gg 1$, the form factor $P(q)$ for spheres has periodic roots at $q R=\frac{3 \pi}{2}, \frac{5 \pi}{2}$, $\frac{7 \pi}{2}, \ldots$ and takes the form:

$$
P(q) \approx\left(\frac{3}{(q R)^{2}}\right)^{2}
$$

By substituting these values for $P(q)$ in Eqn. 4.6 , the intensity can be written as:

$$
I(q)=(\Delta \rho)^{2} V_{p}^{2} P(q)=(\Delta \rho)^{2} V_{p}^{2} \frac{9}{q^{4} R^{4}}
$$


Considering the relation between $V_{p}$ and the surface of a sphere $S_{p}$ to be $V_{p}^{2}=$ $\left[\left(\frac{4 \pi}{3} R^{3}\right)^{2}\right]=\left(\frac{4 \pi}{9}\right) R^{4} S_{p}$, we get:

$$
I(q)=\frac{2 \pi(\Delta \rho)^{2}}{q^{4}} S_{p}
$$

Therefore, the SAXS intensity in the short wavelength limit is inversely proportional to the fourth power of $q$ and in the so-called "Porod region", the sample is not to be considered at an atomistic level; one rather uses a continuum description in terms of an electron density. Data at high angles are assumed to follow a linear plot in $q^{4} I(q)$ against $q^{4}$ coordinates: $q^{4} I(q) \approx \mathrm{B} q^{4}+\mathrm{A}$, and by subtracting the constant B from $I(q)$, scattering of the corresponding homogeneous body (such as a sphere, disc or a rod) can be approximated [55]. This approximation is generally true for particles with different geometry (although derived from spheres) since it gives information of the surface and not at the atomistic level. Although the standard form of the Porod-Debye law is sensitive in the context of a compact domain bounded by a smooth surface and sharp interfaces, a variation in the intensity can also be attributed to the particle dimensionality. This dependence or in other words, the deviations from the Porod regime can be extended to the generalized form of fractal geometry [86, 87]. The word fractal was coined by Mandelbrot (1975) which is derived from the latin word fractus for broken. The non-integer exponents of the power law can be expressed as deviations in systems with nonPorod like behavior and is examined in detail for the results of the continuous flow experiments in Sec. 4.4 .1

\section{Scale-Invariance of the Surface in Proteins}

Extensive study of the vimentin assembly is prevalent which give quantitative analysis of the size parameter and shape. But, there is limited study on the dynamics of the surface during the assembly and aggregation process of vimentin, mainly undertaken by Brennich et al. with the "core and corona model" [51] and Dammann et al. with the competitive binding due to counter-ion condensation [54]. However, studies on scale-invariance such as the identification of fractal dimensions on protein exteriors is still in a nascent stage, especially in the determination of surface roughness of IFs. During assembly, the smaller subunits evolve into three-dimensional structures whose surface and interfaces are often rough. The formation process of these three-dimensional structures is based on physico- 

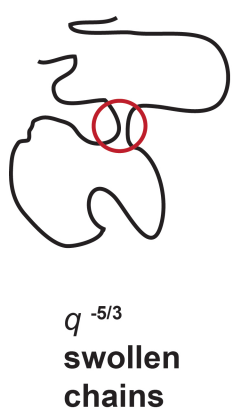

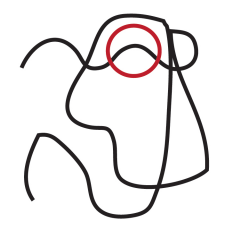

$q^{-2}$ gaussian chains

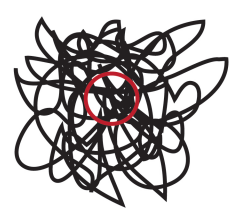

$q^{-3}$

clustered

network

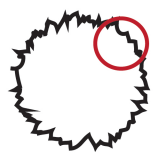

$q^{-3}$

rough

surface

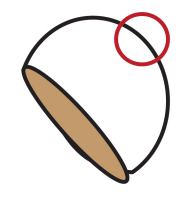

$q^{-4}$

smooth

surface

Figure 4.3.: Sketch of the correlation between exponents and fractals. The first three images account for the exponents obtained to explain the mass fractal dimensions and the $4^{\text {th }}$ and the $5^{\text {th }}$ image corresponds to the surface fractal dimensions. Figure is based on [88].

chemical parameters such as electrostatic potential as discussed in Sec. 2.5. In order to elucidate the surface roughness of the protein exterior, the most important region of interest is the accessible surface area, for which several techniques can be used. To quantify the roughness, fractal dimension is an index of characterization of surface topography of a protein, accurately [89]. One such technique that offers

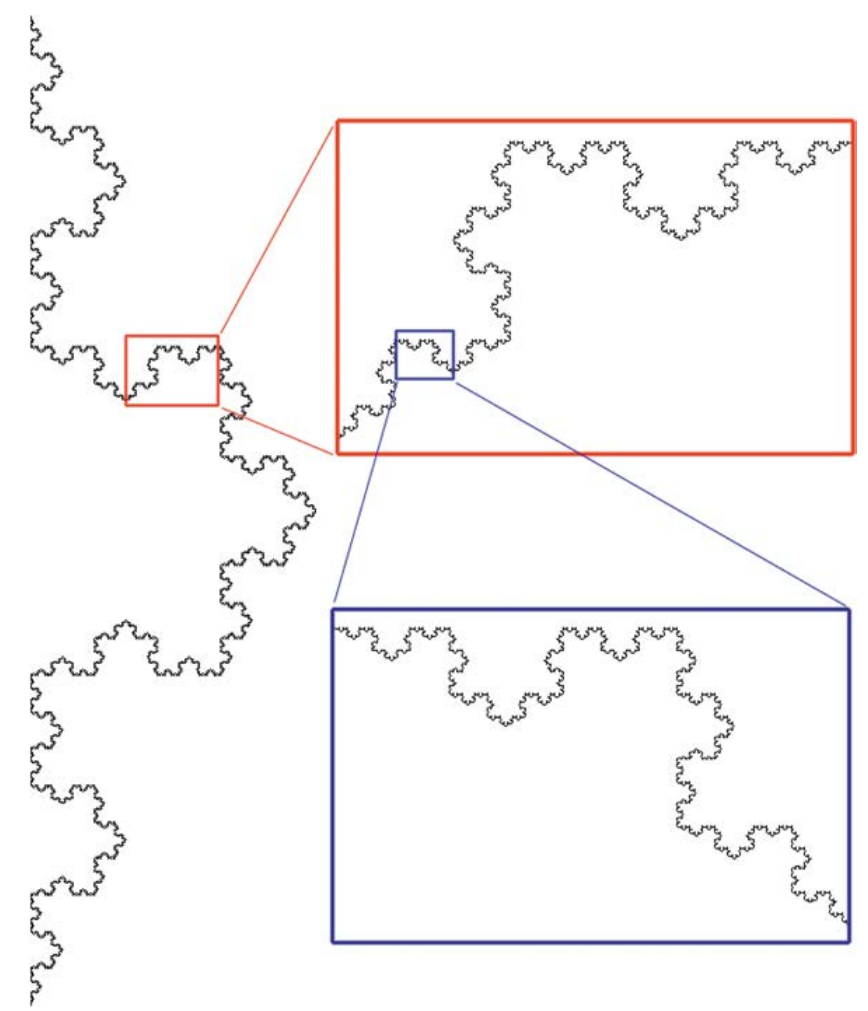

Figure 4.4.: Demonstration of geometric self-similarity. Figure is adapted from [89]. 
the access of surface and interface roughness is SAXS, which by choosing the correct resolution or $q$-range and employing the Porod's theory of asymptotic decay [83], gives information of the exterior of the protein. A representative sketch of the obtained surface topology is shown in Fig. 4.3. The obtained fractal dimensions, which signify surface roughness, are nothing but a scale of roughness that exists over several magnitudes as depicted in Fig. 4.4. This condition is called "selfsimilarity". A quantitative analysis and interpretation of the surface roughness in vimentin is extensively explored in Sec. 4.4.1 with respect to fractal dimensions.

\subsection{Experimental Setups at Synchrotrons}

SAXS experiments were performed at different beamlines of different synchrotron facilities: the ID13 beamline at the European Synchrotron Radiation Facility (ESRF, Grenoble, France), the AustroSAXS beamline at ELETTRA (Trieste, Italy) and the coherent-SAXS (cSAXS) beamline at Swiss Light Source (SLS) at the Paul Scherrer Institute (PSI, Villigen, Switzerland).

Specific beamlines were chosen for the experiments since scanning a micro-channel requires X-ray spot sizes which is of the micron size and are smaller than the channel width to avoid parasitic scattering. In this section, each beamline setup from which data presented in this thesis will be described and a comparison will be drawn with respect to the different experiments.

\subsubsection{ID13, ESRF}

Measurements were performed at the experimental hutch II of ID13 as sketched in Fig. 4.5 which provides the micron-sized focused beam [90]. The undulator beam is monochromatized by liquid nitrogen cooled $\mathrm{Si}(111)$ double monochromator and the beam mode is of uniform multibunch characteristics with a photon energy of $\approx 12.5 \mathrm{keV}$. The micro-hutch beam is pre-focused with refractive beryllium lenses and a defining collimator. The focusing of micron-sized beam onto the sample is further done with Kirkpatrick-Baez (KB) mirrors. The microfluidic devices are mounted on a custom-made aluminum sample holder which is placed on the $x-y-z$ motorized sample manipulator. Behind the sample stage, a beamstop is employed to block out the primary beam intensity. A visible light microscope is used for sample alignment prior to the X-ray measurements. The objective of the microscope is moved into the beam path and the focus is calibrated to coincide with the X-ray focus. This facilitates finding the focal plane of the X-ray beam 


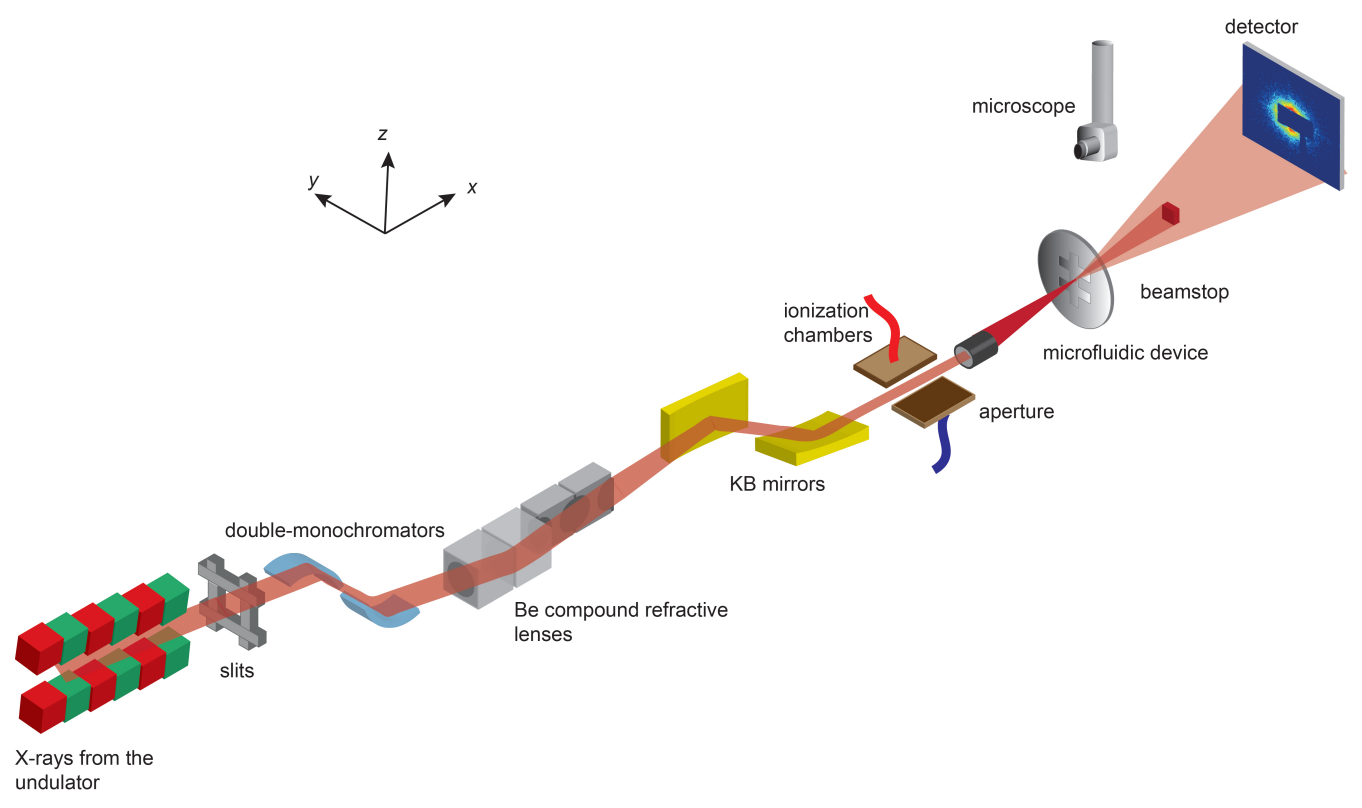

Figure 4.5.: Schematic diagram of the beamline components at ID13 experimental hutch II, ESRF.

for a region-of-interest for all samples. All experiments were performed at room temperature.

For the continuous micro-flow experiment, the beam size was $5 \times 5 \mu \mathrm{m}^{2}$ (horizontal $x$ vertical) and the sample-to-detector distance was $0.52 \mathrm{~m}$. Data were collected with a FReLoN $4 \mathrm{M}$ camera $\left(2048 \times 2048\right.$ pixels; $24 \times 24 \mu \mathrm{m}^{2}$ pixel size; 16-bit readout; $70 \mathrm{~mm}$ Kodak CCD, developed by the ESRF detector group) and the exposure time for each measurement position was $5 \mathrm{~s}$. Consequently, the $q_{r}$-range was $0.24 \mathrm{~nm}^{-1}-9.21 \mathrm{~nm}^{-1}$.

For the droplet experiment of vimentin aggregation, the beam size was $5 \times 3 \mu \mathrm{m}^{2}$ (horizontal $x$ vertical) and the sample-to-detector distance was $0.9928 \mathrm{~m}$ and the $q_{r}$-range was $0.0035 \mathrm{~nm}^{-1}-1.68 \mathrm{~nm}^{-1}$. Data were collected with MAXIPIX detector $\left(516 \times 516\right.$ pixels; $55 \times 55 \mu \mathrm{m}^{2}$ pixel size; fast readout, photon-counting pixel detector system developed by ESRF detector group). Short exposure times of $10 \mathrm{~ms}$ with $3 \mathrm{~ms}$ dead time at every measurement position were employed. The fast acquisition mode for droplets was possible due to the read-out capability of the detector.

For the droplet experiment of vimentin assembly, measurements are performed using a focused beam of $8 \times 10 \mu \mathrm{m}^{2}$ (horizontal $\mathrm{x}$ vertical). The undulator beam 
is monochromatized by a liquid nitrogen cooled $\mathrm{Si}(111)$ double monochromator and the photon energy is $13 \mathrm{keV}$. The white beam is pre-focused by large aperture beryllium lenses. In the experimental hutch, the beam is focused by a set of 2 Beryllium lenses in a transfocator (i.e., two automatic lens changers) and collimated by 3 apertures. The sample to detector distance is $0.76642 \mathrm{~m}$ providing a qr range of $0.42 \mathrm{~nm}^{-1}-2.53 \mathrm{~nm}^{-1}$. Data are collected with an EIGER 4M detector (2070 x 2167 pixels, $75 \mu \mathrm{m}^{2}$ pixel size, Dectris Ltd., Baden, Switzerland). Exposure times of $10 \mathrm{~ms}$ with $3 \mathrm{~ms}$ dead time at every measurement position are employed with a total exposure of $60.5 \mathrm{~s}$ at each point. Data acquisition is repeated 5 times at each position.

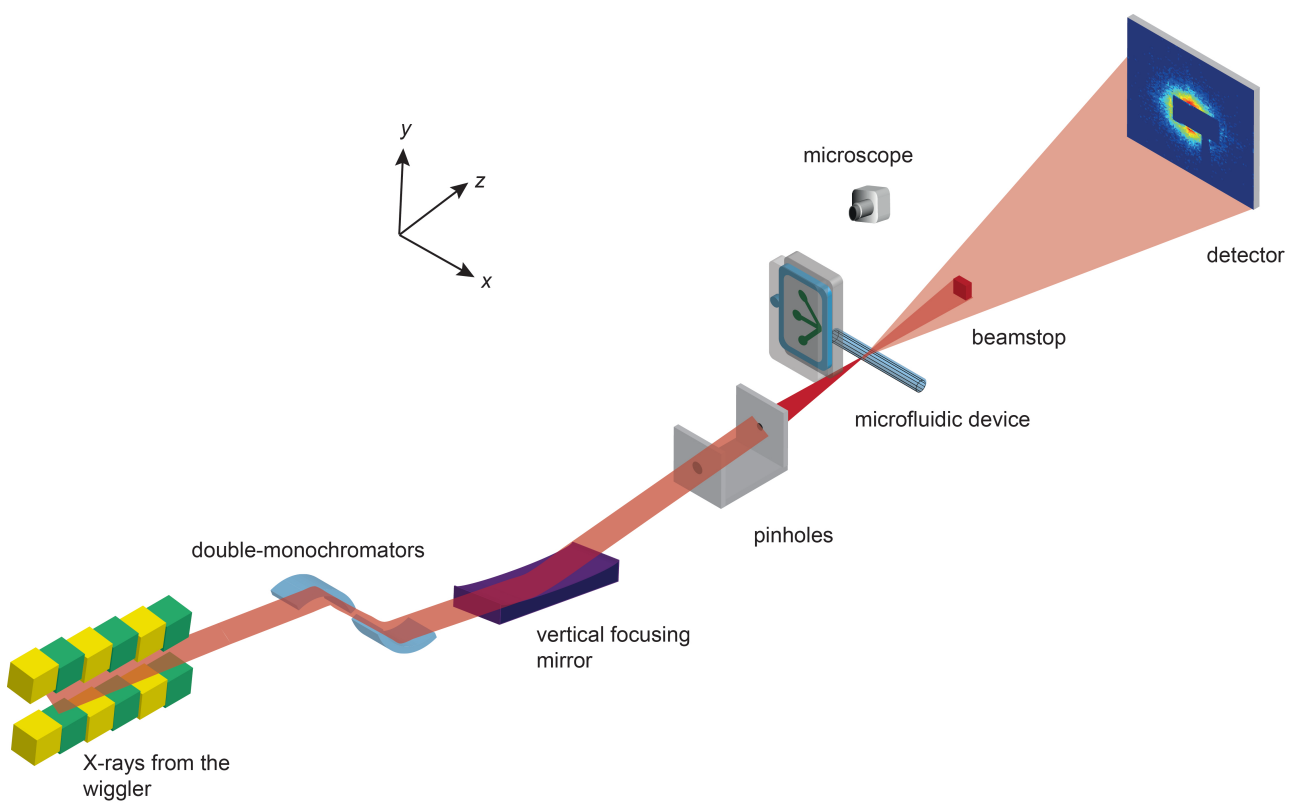

Figure 4.6.: Schematic diagram of the beamline components at AustroSAXS, ELETTRA.

\subsubsection{AustroSAXS, ELETTRA Sincrotrone Trieste}

At the Austrian beamline - "AustroSAXS", measurements were taken in the microfocus beam mode [91] and a sketch of the essential components is shown in Fig. 4.6. The photon source is the 57-pole wiggler from which the SAXS beamline accepts discrete energy of $8 \mathrm{keV}$. The beamline optics consists of a flat, asymmetriccut $\mathrm{Si}(111)$ double crystal monochromator and a double focusing toroidal mirror for pre-focussing. Since further focusing was unavailable, the beam was trimmed to a necessary size by employing a pin-hole. The size of the pin-hole was $50 \mu \mathrm{m} \mathrm{di-}$ 
ameter which defined the spot size on the sample and the pin-hole alignment was motorized. The microfluidic device was mounted on a custom-made aluminum sample holder suited for the $x-y$ sample stage manipulator. A portable visible light microscope was used for sample alignment with the beam before measurements. The beamstop was placed after the sample stage to block the primary beam intensity and the photodiode was placed to collect the transmitted intensities. The scattered intensities were detected on PILATUS 1M, a single-photon counting pixel detector $\left(172 \times 172 \mu \mathrm{m}^{2}\right.$ pixel size, $981 \times 1043$ pixels, DECTRIS ${ }^{\circledR}$, Baden, Switzerland). The detector was placed at $1.1 \mathrm{~m}$ and the corresponding $q_{r}$-range accessible was $0.05 \mathrm{~nm}^{-1}-1.58 \mathrm{~nm}^{-1}$. Glassy carbon and silver behenate were used as calibrants for alignment and for determining the sample-to-detector distance.

For the droplet experiment, the acquisition and measurement technique was based on the idea of Stehle et al. [92], where scattered intensities of several droplets in flow were averaged over the total exposure time of $10 \mathrm{~min}$.

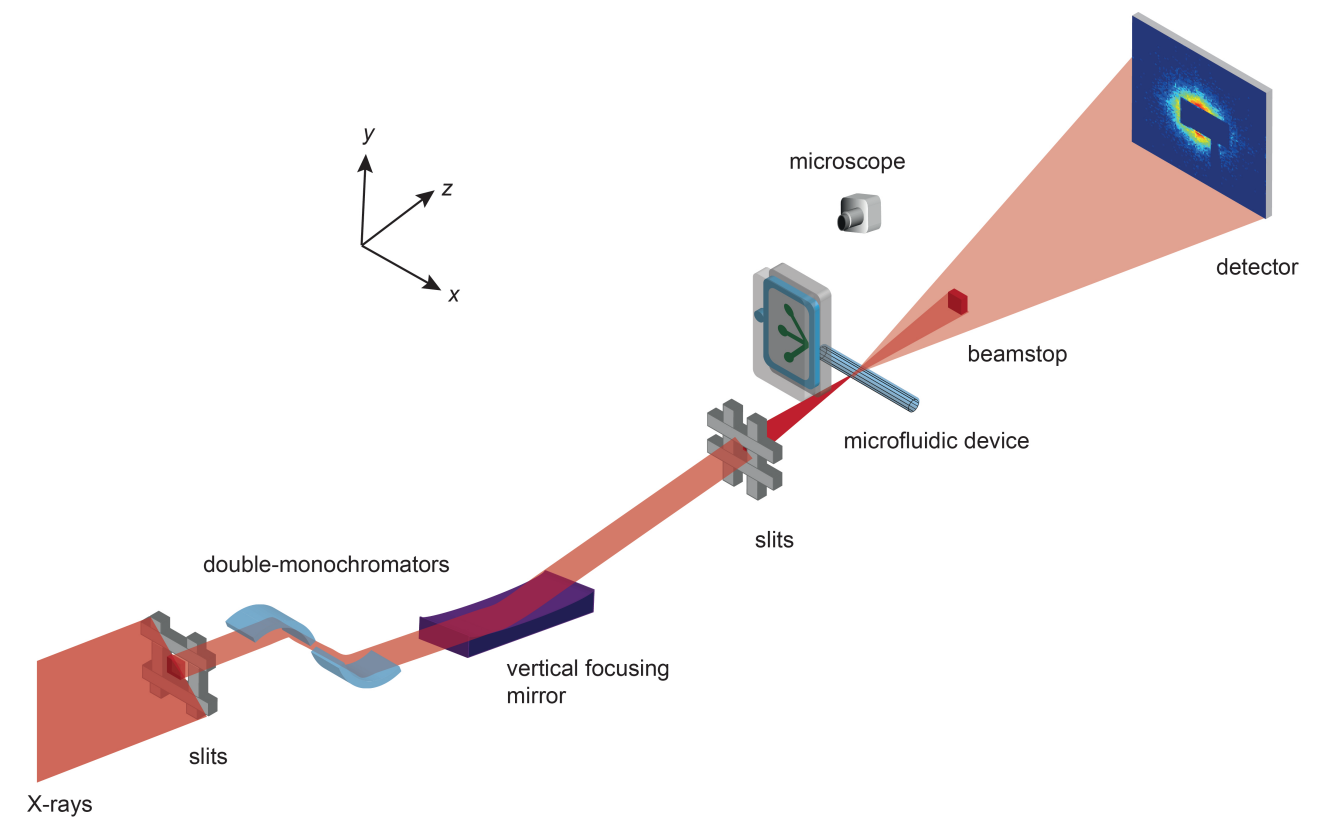

Figure 4.7.: Schematic diagram of the beamline components at cSAXS, SLS. 


\subsection{3 cSAXS, SLS}

The schematic representation of the cSAXS beamline is depicted in Fig. 4.7 where the undulator beam was monochromatized by a liquid nitrogen cooled $\mathrm{Si}(111)$ double crystal monochromator to a defined photon energy of $11.2 \mathrm{keV}$ X-ray beam [93]. The beam is pre-focused with a focusing mirror which rejects higher harmonics and focuses in the vertical direction and slits are used to further trim the beam to a necessary size. In the experiment presented in this thesis, the beam was focused to about a beam size of $30 \times 10 \mu \mathrm{m}^{2}$ (horizontal $\mathrm{x}$ vertical) and was not focused to a spot but an extended focal length. The microfluidic device is mounted on a custom-made aluminum sample holder which is placed onto an $x-y$ motorized sample manipulator. The microfluidic channel is aligned in the $\mathrm{X}$-ray focus using a visible light microscope. The sample can be aligned in the microscope and then moved in-front of the X-ray focus since a calibrated distance between the two exists. Behind the sample, there is a $7 \mathrm{~m}$ flight tube filled with helium with a long-nose leading upto the sample position. The entrance window is a thin sheet of mica, separating the flight tube vacuum of a few mbar from the surrounding air and the primary intensities are blocked by a beamstop in the flight tube.

The total sample-to-detector distance was calibrated using glassy carbon and Silver behenate and in the current experiment was $7.2819 \mathrm{~m}$. The scattered intensities were recorded on PILATUS 2M, a single photon counting detector (1475 x 1679 pixels of $172 \times 172 \mu \mathrm{m}^{2}$ pixel size, DECTRIS ${ }^{\circledR}$, Baden, Switzerland). In the droplet experiment, the acquisition and measurement technique followed was similar to the one employed in Sec. 4.3.2.

\subsubsection{Comparison of the Different Setups}

A comparison of the different setups used at the different beamlines are presented in this section. The main differences constitute the specific scientific question that is addressed in each of the experiments, role of the beam flux, size and intensity conditions adapted, measurement techniques and the beamline components used i.e., such as focusing optics and detectors.

At the ID13 beamline, the experimental hutch II was operational for two microfluidics experiments whose results are presented in this thesis. The first one dealt with the studies involving effect of mono- and divalent salts on vimentin assembly and aggregation in continuous flow microfluidics. Towards this end, a microfocused sized beam was adopted which could probe a jet focused to a few microns 
to match the length scales with a channel geometry presented in Sec. 3.3.1. The second experiment involved measuring unique aqueous-in-oil droplets in flow where the aggregation of vimentin due to the presence of a divalent salt was tracked using a device as explained in Sec. 4.5. A microfocused beam was also employed here. At both the AustroSAXS beamline and the cSAXS beamline, the scientific question to be addressed was the assembly of vimentin due to the influence of monovalent salt in droplets using state-of-the-art PDMS-capillary device mentioned in Sec. 4.5. Comparatively, the beamsize at the AustroSAXS beamline was focused with a pinhole thus losing a lot of primary beamflux. The other beamlines had focussing optics that were either motorized slits or KB mirrors which rendered a clean and a high intense beam. The measurement techniques were different at every experiment. The technique adopted to scan the the continuous flow experiment at ID13 is based on probing a specific region of interest in defined step sizes, thus rendering a composite image mimicking the channel. For the droplet experiment at ID13, short exposure times of $10 \mathrm{~ms}$ with $3 \mathrm{~ms}$ dead time were employed. We used a micro-focused X-ray beam with dimensions of $5 \mu \mathrm{m} \times 5$ $\mu \mathrm{m}$ which was incident at different points on the capillary whilst the droplets (dimension of about $\approx 120 \mu \mathrm{m} \times 80 \mu \mathrm{m}(\mathrm{h} \times \mathrm{v})$ ) flowed by. Due to the small beam size and the short exposure time, several acquisitions per flowing droplet were possible. This strategy allowed us to distinguish clearly between the aqueous and oil phase giving us better signal-to-noise ratio. At the AustroSAXS and cSAXS beam-times, several droplets were allowed to flow by and the average signal over several exposures (minutes at AustroSAXS and seconds at cSAXS) were taken into consideration at one single point. Maxipix was used for its fast-frame rate at ID13 at both the continuous and droplet flow experiments. Pilatus photon counting detectors were used at both AustroSAXS and cSAXS for averaging over several flowing droplets.

\subsection{Continuous-Flow Experiments}

\subsubsection{Results from Experiments}

Parts of this section is published in the journal Biomicrofludics [63] and is reproduced here with permission.

We employ SAXS with a micro-focused beam to study the structural changes in 
vimentin during assembly in the presence of ions and parts of this section are prepared for a publication [63].Since SAXS is an inherently "slow" technique due to the required exposure times for weakly scattering biological molecules $(5 \mathrm{~s}$ in this specific case), we combine the x-ray method with continuous flow microfluidics. The laminar flow conditions (the maximum Reynolds number is $\mathcal{R} e_{\max }=0.4$ ) in the small channels ensure that by exposing a specific position, we ensembleaverage identical states of the assembly process. Therefore, the temporal resolution is given by the distance between the measurement positions along the outlet channel rather than the exposure time. An additional advantage of the continuous flow is the reduced radiation damage affecting the protein since it is continuously passing by the x-ray beam. The microfocused x-ray beam $(5 \mu \mathrm{m} \times 5 \mu \mathrm{m})$ has dimensions smaller than the channel width $(300 \mu \mathrm{m})$ leading to high spatial resolution in real space.

The device is scanned through the beam in a rectangular lattice (step size $\Delta x \times \Delta y$ is $100 \mu \mathrm{m} \times 30 \mu \mathrm{m}$ ) to localize the protein stream in the center of the channel. This experimental strategy is shown in a composite image of the individual SAXS patterns (Fig. 4.8). In Fig. 4.8 the aspect ratio $x / y$ of the SAXS patterns is shown in a distorted way in order to match the anisotropic step sizes used in the scanning. In Fig. 4.8 the SAXS patterns are shown with their real aspect ratio. The total intensity of each pattern along the main flow axis of the device is integrated, normalized by the respective protein concentration derived from the simulations and plotted against the position $l$ in Fig. $4.8 \mathrm{p}$. The representative measurement positions which we refer to in the analysis further down are circled in Fig. 4.8 a. As color code we use blue for vimentin in the presence of only $\mathrm{KCl}$ and purple for vimentin in the presence of both $\mathrm{KCl}$ and $\mathrm{MgCl}_{2}$ [63]. The composite representation of the individual SAXS patterns presented in Fig. 4.8 a shows that the channel can be precisely mapped by employing the 5 - $\mu$ m-beam. The intensity (inverted gray scale) is increased in the central stream where the protein is located (see also detail in Fig. 4.8k). Furthermore, when moving along the $x$-direction downstream the flow, the intensity increases upon addition of $\mathrm{KCl}$ (first set of lateral inlets) and again upon the addition of $\mathrm{MgCl}_{2}$ (second set of lateral inlets). Fig. 4.8p shows an initial increase in the total intensity when $\mathrm{KCl}$ is added. This increase is followed by a decrease, which could be due to hydrodynamic shaping of the protein stream. At the second lateral inlet, where $\mathrm{MgCl}_{2}$ is added, we observe a large jump in intensity [63].

We choose SAXS patterns, as indicated by the circles in Fig. 4.8a, for further anal- 


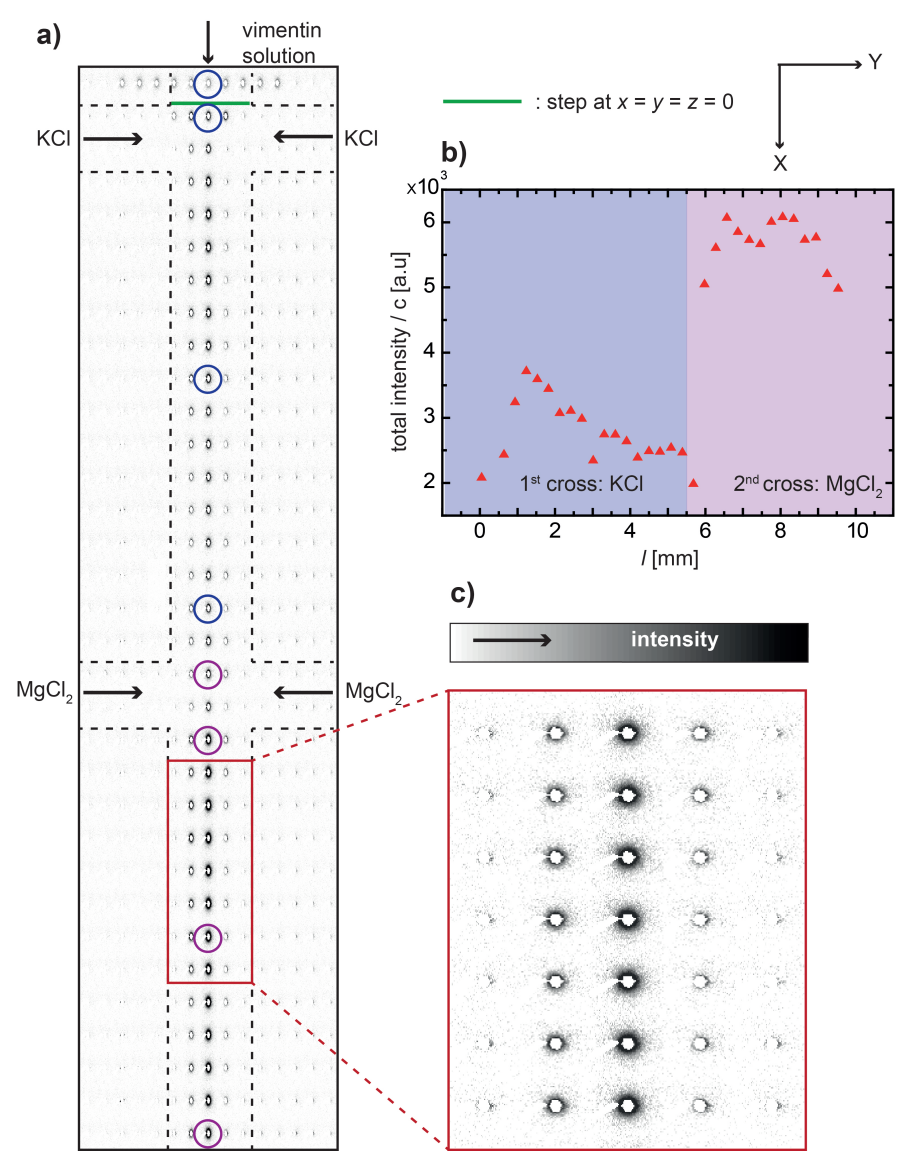

Figure 4.8.: X-ray measurement strategy for continuous flow experiments (a) Composite image of individual SAXS patterns taken at different positions in the channel; inverted gray scale, i.e. darker regions refer to increased intensity; colored circles refer to the measurement positions depicted in Fig. 4.9p. (b) The total scattered intensity along the main flow direction normalized by the protein concentration. (c) Zoom-in of a detail from a. Note that whereas in a the aspect ratio of the individual SAXS patterns have been distorted in order to match the aspect ratio of the channel (line and column spacing in the scan are different), in b the SAXS patterns are displayed as recorded.Figure taken from [63] with permission.

ysis. The data are integrated azimuthally and plotted against the scattering vector $q_{r}$, as shown in Fig. 4.9. The individual $I(q)$ curves are shifted with respect to each other by a factor of 10 for better visibility. In the past[35], we have analyzed scattering from vimentin assembled in microflow at small $q_{r}$ values up to about $0.5 \mathrm{~nm}^{-1}$ before reaching the noise level and performed a Guinier analysis of the data. Here, with a complementary setup at a different beamline, we access higher $q_{r}$ values before reaching the noise level. We therefore analyze the data according 

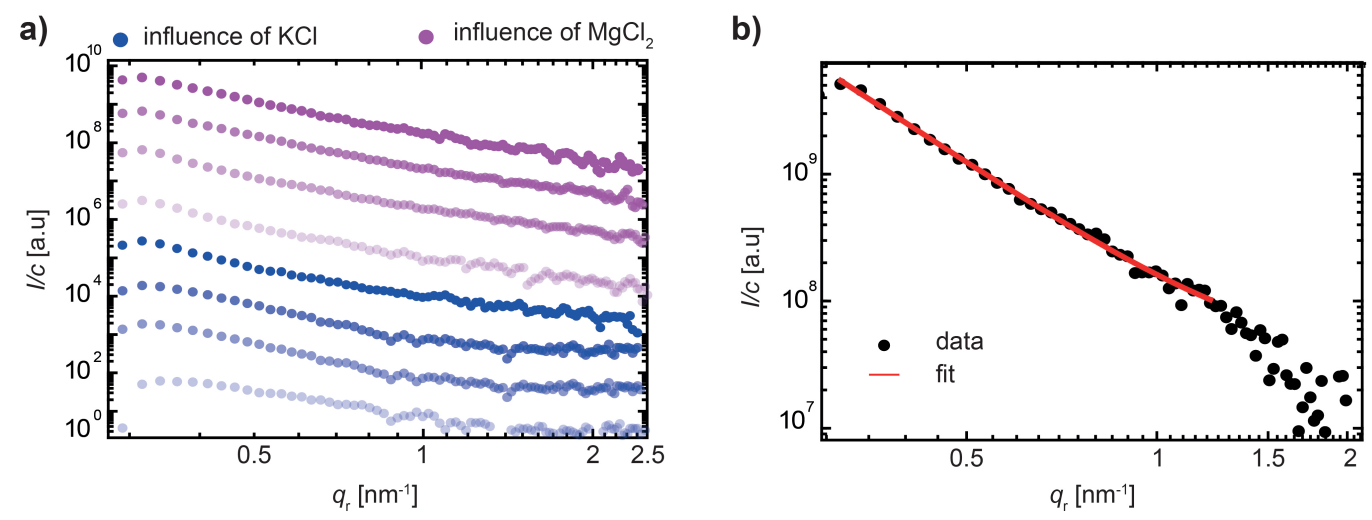

Figure 4.9.: Experimental results of SAXS for continuous flow (a) Double-logarithmic plot of scattering curves. The blue data curves refer to vimentin in the presence of only $\mathrm{KCl}$ and the purple curves refer to vimentin in the presence of both $\mathrm{KCl}$ and $\mathrm{MgCl}_{2}$ as depicted in Fig. $4.8 \mathrm{a}$. The same color code is used throughout the figure and the text. (c) Representative data curve with the fit range to determine power law exponents. Figure taken from [63] with permission.

to Porod's law of asymptotic decay [83]:

$$
I(q) \propto q^{d_{f}}
$$

By plotting the intensities in a double-logarithmic representation, the slope is directly associated with the power law exponent $d_{f}$. Interpretation of these values will be discussed further below. The correct range applicable for Porod's law can be determined by plotting $I(q) \times q^{4}$ versus $q^{4}$ and choosing a smooth linear region limited by noise in the higher $q_{r}$. An example is shown in Fig. 4.10. The data are fitted in the $q_{r}$-range between $0.32 \mathrm{~nm}^{-1}$ and $1.2 \mathrm{~nm}^{-1}$ for all the datasets as shown for a representative curve in Fig. $4.9 \mathrm{~b}$. These fits yield exponents from -2.2 to -3.2 for the influence of $\mathrm{KCl}$ and from -3.2 to -3.3 for the influence of $\mathrm{MgCl}_{2}$ for datasets with flow-rates of $80 \mu \mathrm{L} \mathrm{h}^{-1}$ in the lateral inlets and $40 \mu \mathrm{L} \mathrm{h}^{-1}$ in the central inlet. We find (see Fig. 4.11 , red circles, triangles and stars for three individual measurements) a steep decay in $d_{f}$ upon addition of $\mathrm{KCl}$, whereas upon the addition of $\mathrm{MgCl}_{2}$, the exponents remain constant. In comparison, for decreased flow-rates ( $40 \mu \mathrm{L} \mathrm{h}^{-1}$ in the lateral inlets and $20 \mu \mathrm{L} \mathrm{h}^{-1}$ in the central inlet), the exponents decrease in a more pronounced way when KCL is added (dark green circles). For increased flow-rates (160 $\mu \mathrm{L} \mathrm{h}^{-1}$ in the lateral inlets and $80 \mu \mathrm{L} \mathrm{h}^{-1}$ in the central inlet), the exponents trace a less steep decay (light green circles)[63].

The streamline tracking presented in the previous section allows us to translate 


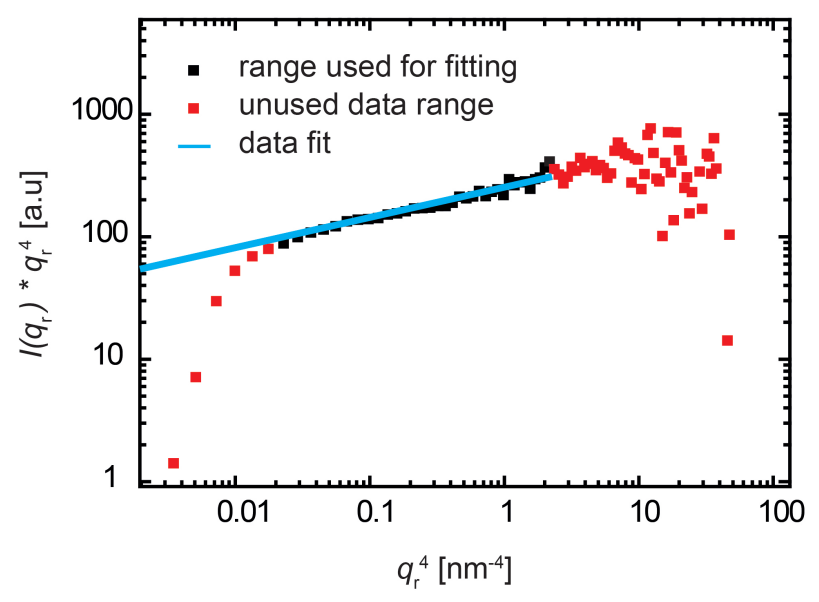

Figure 4.10.: Fit determining the correct $q_{r}$ range for Porod's analysis for $\mathbf{X}$-ray data. The $I(q) \times q^{4}$ versus $q^{4}$ representation is called the Porod-Debye plot and is used to determine the fit range for the analysis. The slope of the fit line must be positive. The goal is to find a linear region by excluding the first few points arising from the beamstop at lower $q$-range and noise at higher $q$-range [80 94]. Figure taken from [63] with permission.

positions in the channels into flow times as shown in Fig. $3.13 \mathrm{p}$. When plotting the exponents versus time, as shown in Fig. 4.11p, we find that the data for the different sets of flow velocities do move closer together and for the two faster velocities (160:80 and 80:40, light green and red) they even collapse, but not for the slowest velocity (40:20, dark green). Note that in Fig. 4.11 the position $l=$ 0 deviates slightly (by about $150 \mu \mathrm{m}$ ) from the position where the protein first encounters a threshold concentration of $10 \mathrm{mM} \mathrm{KCl}$. By contrast, when plotting the exponents against the concentration of $\mathrm{KCl}$ in Fig. $4.13 \mathrm{a}$, the data collapse into two groups, one with larger values at 0 and one with smaller values at about 70 $\mathrm{mM}$. A plot of the concentration of KCL against time (Fig. 4.13p) reveals a fast "jump" from 0 to $70 \mathrm{mM}$ salt after about $3 \mathrm{~s}$ and thus explains this observation. We therefore conclude that while fast, the assembly reaction is not instantaneous and the temporal evolution is resolved in Fig. 4.11p [63]. 
a)

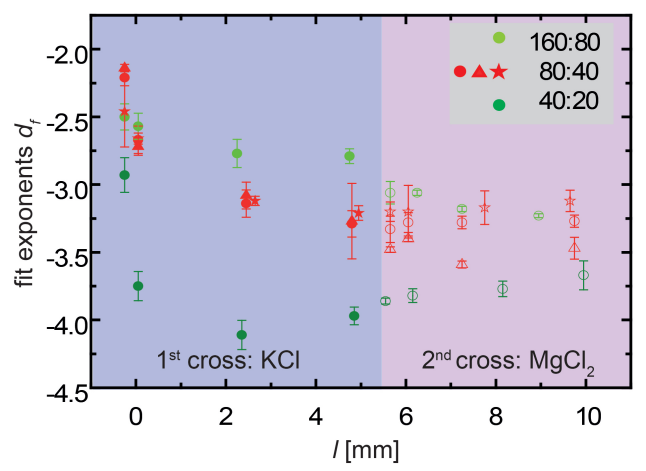

b)

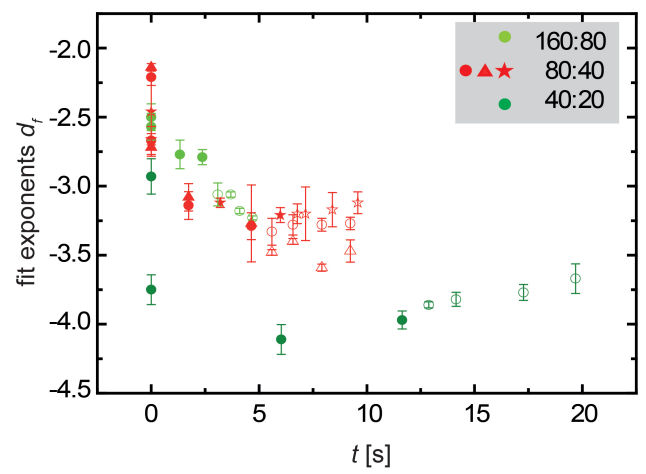

Figure 4.11.: Fit exponents from Porod's analysis for continuous flow experiments (a) By fitting the X-ray data to Porod's equation for the intensity decay, we determine the power law exponents, which decrease during the influence of $\mathrm{KCl}$ and remain stable at the employed $\mathrm{MgCl}_{2}$ concentrations with respect to the length of the channel. The legend for the data points is the ratio of the flow speeds of the lateral inlets to the central inlet in $\mu \mathrm{L} \mathrm{h}^{-1}$, e.g. 160:80 stands for $160 \mu \mathrm{L} \mathrm{h}^{-1}$ in the lateral inlets and $80 \mu \mathrm{L} \mathrm{h}^{-1}$ in the central inlet (b) Fit exponents $d_{f}$ for each dataset with respect to time scales for different flow speeds corresponding to Fig. 3.14 The explanation for the legend follows as in (a). Figure taken from [63] with permission.

\subsubsection{Data Interpretation and Discussion}

a)

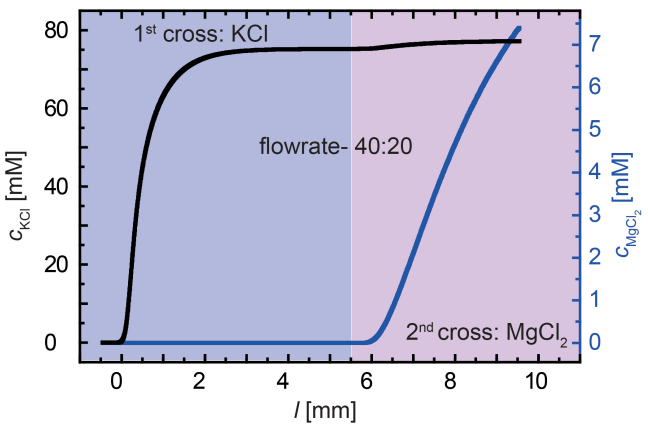

b)

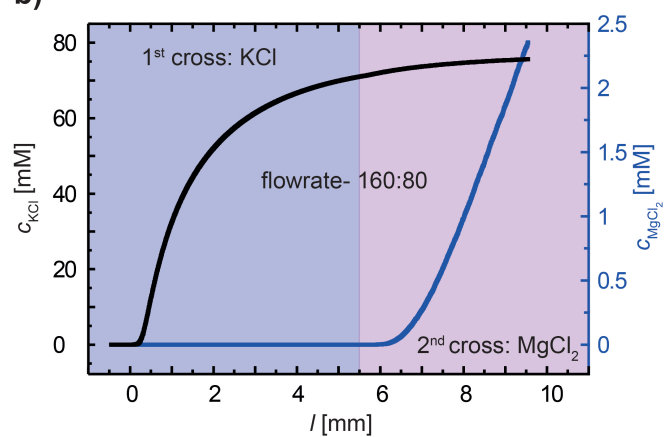

Figure 4.12.: Results derived from the FEM simulations for flowrates 40:20 and 160:80 for the central stream line $(y=z=0)$ (a) Concentrations of $\mathrm{KCl}$ and $\mathrm{MgCl}_{2}$ for 40:20. (b) Concentrations of $\mathrm{KCl}$ and $\mathrm{MgCl}_{2}$ for 160:80. Figure taken from [63] with permission.

By combining experimental microfluidics and numerical FEM simulations, we obtain precise knowledge of the flow conditions in the microfluidic device. The continuous flow approach offers high control of the mixing conditions which are completely governed by diffusion in the laminar flow. It is well known that addition of $\mathrm{KCl}$ initiates the assembly of tetrameric complexes into bona fide IFs in 
vitro. We and others have also shown that $\mathrm{MgCl}_{2}$ influences the nanostructure of assembled filaments as well [37, 38, 39]. However, in previous SAXS studies, we mixed the ions either each separately with the vimentin or at once, but not in sequence [63].

Using microfluidics, we are able to mix in ions successively in a precise and controlled manner as previously shown in a microscopy study[52, 53, 54]. Here, we take this approach to the nanometer scale by employing SAXS and mixing in monovalent $\mathrm{KCl}$ first and thereafter divalent $\mathrm{MgCl}_{2}$. The length scales probed by our SAXS setup (approximately 3 to $60 \mathrm{~nm}$ ) and the time scales defined by the microfluidics parameters (up to $20 \mathrm{~s}$ ) are adapted to the lateral assembly of vimentin tetramers to ULFs. We observe an intensity increase along the center stream upon addition of $\mathrm{KCl}$, which is indicative of vimentin assembly and thus stronger overall scattering. Mixing in $\mathrm{MgCl}_{2}$ leads to an even stronger intensity increase which can be attributed to thickening of the ULFs or to the formation of larger aggregates. Moreover, we have observed in the past for vimentin, that $\mathrm{MgCl}_{2}$ accumulates in the unstructured tail regions of the filaments, which are presented at the surface of the filament core and thus leads to higher relative scattering [37]; the present data are in agreement with these results [63].

In order to understand the structural-molecular changes, we analyze the Porod exponents derived from the scattering curves. The standard Porod-Debye law [83, 95] for the interpretation of the exponents requires a compact domain with a smooth surface and sharp interface and leads to information of the shape of the scatterers: $d_{f}=-4$ for spheres, -2 for discs and -1 for rods. However, biomolecules do not have such a smooth surface and the theory has been generalized to noninteger values for fractal geometry as mass fractal dimensions [86, 87]. The predictions of this generalized theory are valid for (i) a monodisperse particles and (ii) if the self-similarity conditions prevail over at least one full order of magnitude [86, 96]. Values between -1 and -3 for the exponents $d_{f}$ correspond to the transition of loosely packed, Gaussian-chain mass fractals to densely packed mass fractals. Values between -3 and -4 , by contrast, correspond to ever-smoother surfaces. We are aware that for biological and technical reasons our data do not completely fulfill the two conditions (i) and (ii) above, but still use the theory as a first approximation of the observed molecular processes [63].

Plotting the exponents $d_{f}$ against the channel position (Fig. 4.11a), we show results for three different sets of flow rates and three individual data sets were analyzed in the case of the intermediate flow rates (red circles, triangles and stars). 
Their strong agreement underlines the reproducibility of the microfluidics measurements. From the results of the simulations (see Fig. 3.12 and 3.13 and compare to Fig. 1, 2 from Appendix. C and Fig. 4.12), we know that as the flow rates decrease, the channel length needed for the ions to diffuse into the central stream is shorter and the maximum ion concentration is reached at smaller values for the channel position $l$. Thus, we observe an assembly and smoothening in the presence of $\mathrm{KCl}$ for smaller values of $l$ at the slowest flow rate (dark green data points in Fig. 4.11), represented by a rapid drop in the $d_{f}$ values. The $d_{f}$ values for higher flow rates show a drop as well, albeit not as rapid and not as pronounced. Upon the diffusive addition of $\mathrm{MgCl}_{2}$ up to a concentration of $2.5 \mathrm{mM}, 4 \mathrm{mM}$ or 7 $\mathrm{mM}$ for the different sets of flow rates, respectively, the exponents remain constant indicating no further compaction of the assemblies. We also observe here that the differing maximum ion concentrations reached for the different flow rates do not significantly influence the results [63].

From these data we conclude that a critical concentration of $\mathrm{KCl}$ is necessary

a)

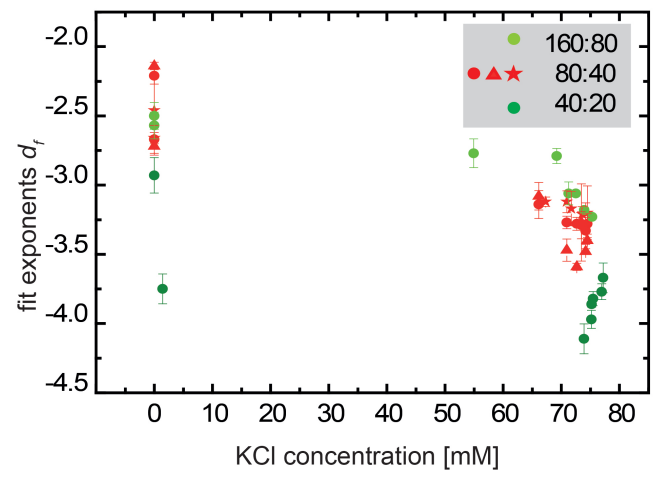

b)

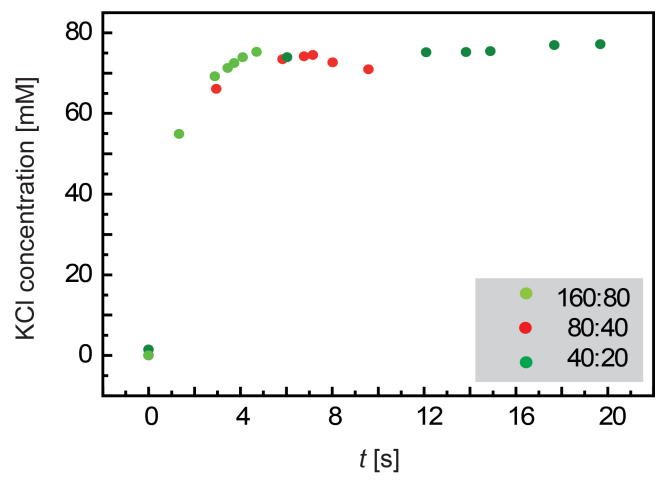

Figure 4.13.: Experimental results of SAXS (a) Fit exponents $d_{f}$ plotted versus the $\mathrm{KCl}$ concentration, which rapidly jumps from 0 to about $70 \mathrm{mM}$ as shown in (b). Figure taken from [63] with permission.

to unblock the formerly unproductive head domains and thus initiate assembly. Assuming a critical concentration of $10 \mathrm{mM}[37]$, it is reached at $l \approx 0.17 \mathrm{~mm}$ for the slowest flow rate and only at $l \approx 0.5 \mathrm{~mm}$ for the highest flow rate. This corresponds very well with the positions where we measure a value for the Porod exponent of $d_{f} \approx 2.7$ for both data sets, which we thus assign to the onset of lateral association of tetramers. Note that a critical protein concentration has not been found so far [34] and ULF formation was observed even for protein concentrations as low as $5 \mu \mathrm{g} \mathrm{mL} \mathrm{m}^{-1}$. 
By contrast, when plotting the same data against the time axis (Fig. 4.11p) we see the development of the nanostructure during a certain time period since first contact with the ions. Interestingly, when plotting the data against time, the curves for the faster flow rates (light green and red data points) collapse, but not the data for the slowest flow rates (dark green data points). At this point we can only speculate, what the reason for this observed behavior may be. Partly this different behavior could be due to the more rapid addition of $\mathrm{KCl}$ and thus initiation of assembly. Another possible explanation could be found in an influence of the flow velocities and associated shear stresses. We do not observe anisotropy in the scattering patterns and thus no orientation effect of the flow on the vimentin assemblies. However, it is possible that the involved shear stresses change the behavior of the protein during assembly and in particular the tails of the monomers, which stick out of the filament body forming a "bottle brush" structure [37], could be influenced. A "slight" alignment of the tetramers by the flow could also influence the assembly speed since it would bring the tetramers already into the right rotational orientations and thus decreases the number of degrees of freedom.

One of the major advantages of the microfluidic geometry used in the present work is the possibility to mix the ions subsequently. In corresponding bulk SAXS experiments, by contrast, all the ions have to be mixed with the tetrameric proteins at once and the end state of the assembly process is measured in glass capillaries[37]. Nevertheless, our present observation of a strong overall intensity increase upon the addition of $\mathrm{MgCl}_{2}$, which is less pronounced for the addition of $\mathrm{KCl}$ supports the idea of a direct, strong interaction of the divalent, but not monovalent ions with the unstructured tail regions of the vimentin filaments [63]. In a different microfluidic setting, employing picoliter drops imaged by fluorescence microscopy, we have previously exposed pre-assembled vimentin filaments to different concentrations of $\mathrm{MgCl}_{2}[52,53,54]$ and observed dynamics of the network formation. In that study we found that a concentration of $10 \mathrm{mM} \mathrm{MgCl}$ represents a sharp threshold, above which vimentin filaments bundle into very dense aggregates on a microscopic scale. Changes on the nanometer scale were not observable by fluorescence microscopy. Here, we focus on lower $\mathrm{MgCl}_{2}$ concentrations, which are closer to physiological conditions, and observe that the surface character of the filaments is not influenced [63]. Transmission electron microscopy (TEM), which is a complementary, nanometer resolution technique, leads to consistent results, where no change in filament morphology could be observed for $\mathrm{MgCl}_{2}$ concentrations up to $4 \mathrm{mM}$ [45]. Macro- and microrheology studies 
show a cross linking of vimentin filament networks in the presence of a few $\mathrm{mM}$ $\mathrm{MgCl}_{2}$ [44, 45], consistent with the increase of overall scattering intensity observed in our present SAXS experiments [63].

To summarize and conclude, we present a microfluidic technique that enables us to functionally activate an assembly-competent protein complex of the IF protein vimentin by addition of ions to non-filamentous vimentin, namely tetramers, and observe the effect in a time resolved manner. The combination of SAXS and microfluidics provides exactly the sub-second time scales and nanometer length scales needed to study the association process of these proteins. Accessing the Porod regime of $q$-resolution enables us to probe the surface structure of the emerging assemblies. We demonstrate the effect of monovalent and divalent ions in two subsequent steps: The addition of $\mathrm{KCl}$ at a critical minimal concentration initiates the lateral association of average eight vimentin tetramers and in parallel smoothens the surface of the protein assemblies. Addition of $\mathrm{MgCl}_{2}$ up to $7 \mathrm{mM}$ does not significantly influence the surface but leads to a further lateral addition of tetramers to the ULFs and to an accumulation of the ions in the side chains which are present on the surface of the filaments. The observed smoothening of the vimentin filaments in statu nascendi, which takes place on the times scale of a few seconds, indicates that this is the time needed by the tetramers from first encounter to the formation of an ordered structure, i.e. a full-width filament unit, which is then ready for further longitudinal association with other ULFs, i.e. for filament elongation. Thus, our combined microfluidics-SAXS experiments reveals the time scale for assembly, which is not a one-step reaction but a process that apparently takes several seconds. Our data for vimentin may have impact on other, alerted IF proteins such as desmin or neurofilaments as well [63].

\subsection{Droplet Experiments}

An enterprising method to study the biological processes in solution is to encapsulate them in pico-litre sized droplets. Here, we investigate the effect of monovalent ions on vimentin assembly and the effect of divalent ions on the aggregation of pre-formed vimentin filaments on the nanometer scale with SAXS. The advantage of this technique is to prevent protein adherence to the channel walls (which otherwise leads to clogs and disrupts the measurement) and to study the kinetics as a function of time. An added advantage of flowing droplets to study protein systems is the reduction of radiation damage. Two challenging steps for vimentin 
studies with X-rays are (i) development of a X-ray and droplet compatible device (ii) an effective measurement technique to image the flowing droplets at a high signal-to-noise ratio. By fabricating a composite PDMS - quartz capillary device which is both X-ray and droplet compatible, we circumvent the first issue and is introduced in detail in Chap. 3, Sec. 3.4.2. Results of the combination of droplets studied with SAXS with different measurement routines are presented in the following sections.

\subsubsection{Results from Measurements at ID13, ESRF}

The challenge of acquiring high-spatial resolution data with respect to the $q$-range from flowing droplets is addressed here by combining a microfocussed X-ray beam with dimensions of $5 \mu \mathrm{m} \times 5 \mu \mathrm{m}$ and a fast acquisition mode. For this purpose, we employ vimentin as a model system and track the early stages of vimentin assembly and aggregation in the presence of $\mathrm{K}^{+}$and $\mathrm{Mg}^{2+}$ confined within droplets. For the vimentin assembly experiment, three aqueous inlets mix the tetrameric vimentin with the $\mathrm{K}^{+}$ions separated by a stream of $2 \mathrm{mM}$ PB. For the aggregation experiment, three aqueous inlets mix the pre-formed vimentin filaments with the $\mathrm{Mg}^{2+}$ ions separated by a stream of $100 \mathrm{mM} \mathrm{KCl}$. the droplets are then pinched-off by the oil phase from adjacent direction as shown in Fig. 4.14(a).

Since solution SAXS of native biological processes is a weakly scattering phenomena, data taken at several positions along the quartz capillary enables us to convert the spatial resolution into time resolution. To enhance the spatial resolution, a microfocussed X-ray beam is incident at short exposure times of $10 \mathrm{~ms}$ with a $3 \mathrm{~ms}$ dead time when the droplets (dimension of about $\approx 120 \mu \mathrm{m} \times 80$ $\mu \mathrm{m} \mathrm{hx} \mathrm{v)} \mathrm{flow} \mathrm{by.} \mathrm{Several} \mathrm{acquisitions} \mathrm{of} \approx 12-15$ exposures are possible per flowing droplet due to the small beam size and the short exposure time as shown in Fig. 4.15(a) (b) and (d). This strategy maps every flowing droplet and there is distinguishable difference between the oil and droplet scattering patterns. When the X-ray beam is incident at the interfaces between the aqueous droplet and the oil, a highly anisotropic scattering pattern is recorded where a noticeable streak develops.

Composite scattering patterns acquired at position ' $x$ ' over time are arranged in rows for better visualization as shown in Fig. 4.15(b). The scattering patterns with low intensity is attributed to arising from oil since they absorb X-rays more strongly than the aqueous droplets. However, a higher intensity is recorded for the edges of the droplets. Observation of the scattering from the different regimes 
a

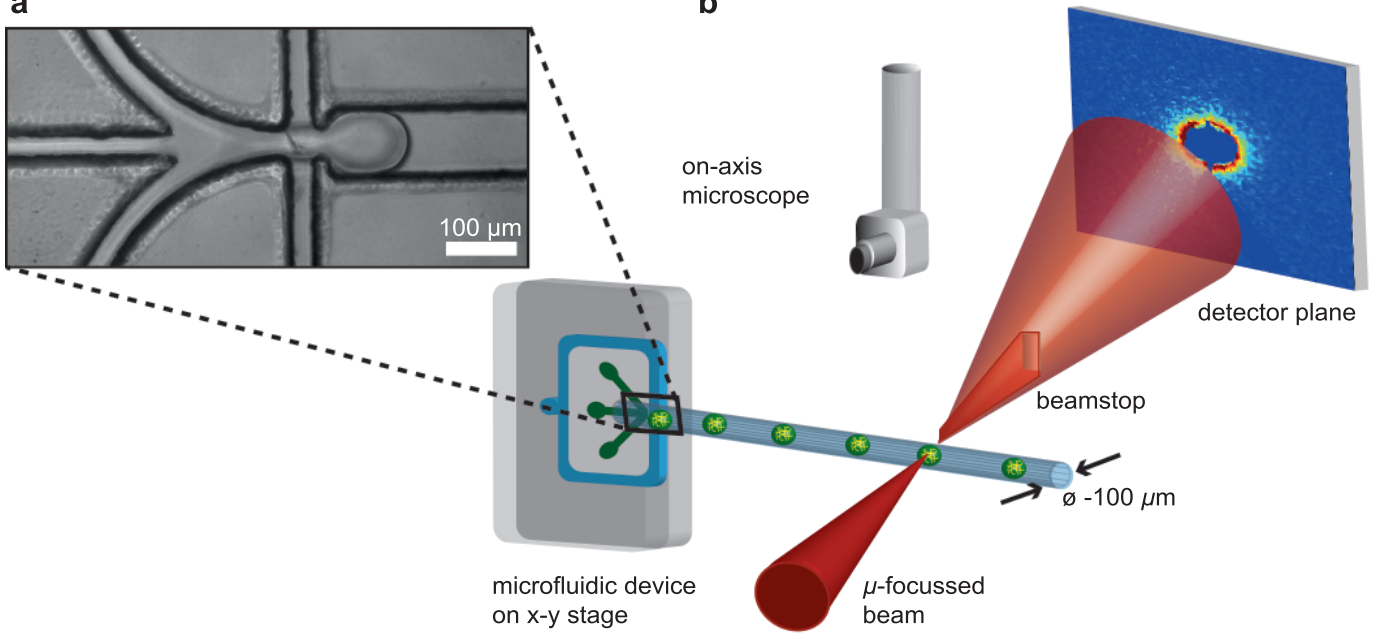

Figure 4.14.: (a) An optical microscopy image of the geometry used for droplet generation where the three aqueous components merge together and are pinched off by the adjoining oil inlets at right angles [52] (b) Schematic of a droplet microfluidic- SAXS experiment, depicting the composite PDMS-capillary device mounted on a $x y$ stage and the $\mathrm{X}$-rays being incident on different positions on the flowing droplets. The on-axis microscope helps in alignment of the device with the focus of the incident beam and the beamstop blocks the intense primary beam. The scattered intensities are recorded on a detector plane rending a 2D scattering pattern. An optical microscopy image of the geometry generating droplets from the PDMS segment as shown in (a)

is increased when the the scattering intensity is summed and presented as an Xray dark field image shown in Fig. 4.15(c).

In order to eliminate the parasitic scattering from oil and the interface of oil and droplet, thresholding of the scattering patterns is done based on the total intensity from the dark field image. By setting the intensity values of the parasitic scattering patterns to zero, the pattern from oil and the edges are discarded and the signal from the aqueous droplets are averaged. All frames between two consecutive streak patterns image the entire droplet end-to-end in the horizontal direction during the course of the flow.

For the vimentin assembly experiment, integrating the now-averaged scattered patterns from several hundreds of droplets, we obtain 1D-scattering profiles along different positions on the capillary which amount to a time-resolution on the order of milliseconds to seconds. The scattered intensities are shown on a doublelogarithmic representation in Fig. 4.16. Since a correction for transmission through the sample is not possible due to geometric and beamline constraints, a scaling of the baselines for the curves is done to compare them. Position 1 corresponds to the 


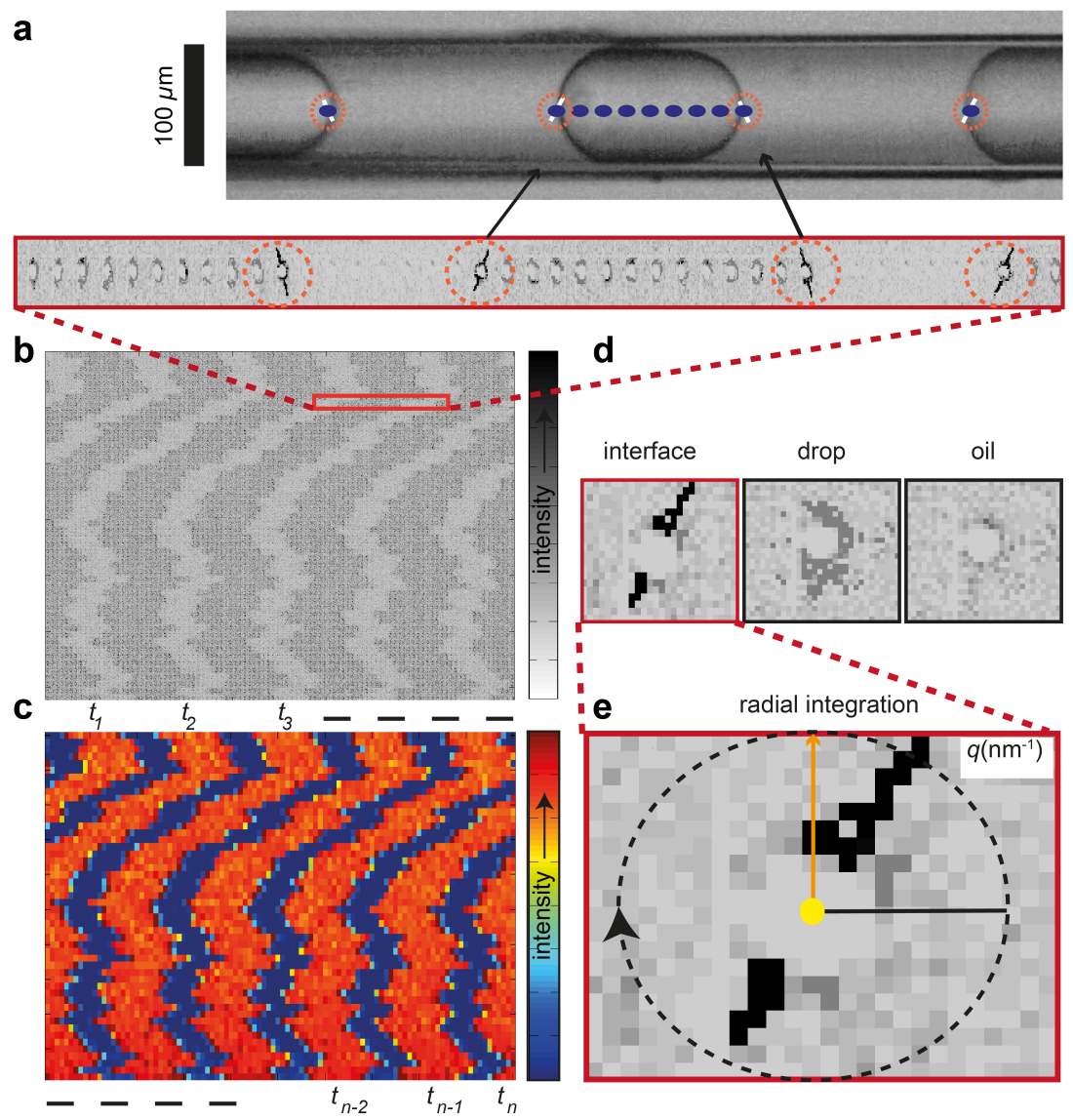

Figure 4.15.: (a) Optical micrograph of a quartz capillary segment from the composite PDMSQuartz capillary device where the monodispersed droplets are flowing by. The "blue" dots correspond to the representative scattering positions in the droplets. A corresponding image of the scattering patterns from the actual droplets are plotted below. (b) Composite scattering images are plotted with respect to time and scattering patterns from approximately 250 droplets are arranged in rows for better visualization. A part of the marked single row is zoomed in (a). (c) X-ray dark-field image of the droplets where the scattering intensity is summed. (d) 2D scattering profiles from the different parts of the measurement are shown where the interface between the oil and droplets produces a strongly anisotropic streak. The signal from the drop is low and the signal from the oil is highly absorbed. (e) Scheme of the radial integration of the $2 \mathrm{D}$ patterns and binning.

closest point after the pinch-off, whereas position 10 corresponds to the last accessible point on the quartz-capillary. The time resolution at position 10 is $\approx 9 \mathrm{~s}$. We can infer that the vimentin assemblies seem to be growing as the overall intensity at lower $q_{r}$ is seen to be increasing.

For the vimentin aggregation experiment, the scattered intensities are shown on a double-logarithmic representation in Fig. 4.17. The intensity curves are observed 


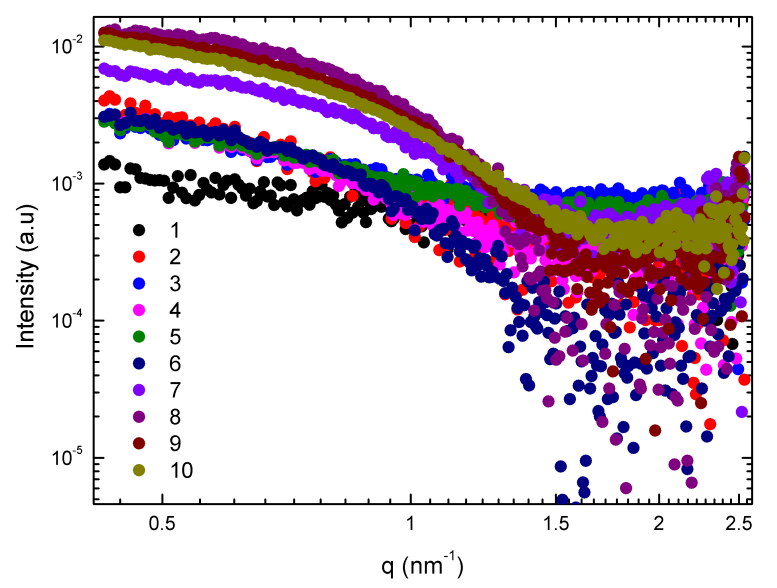

Figure 4.16: Integrated 1D-scattering intensity profiles measured at different positions on the quartz-capillary. Position 1 refers to the first droplet position after the pinch-off. The following positions are measured in chronological order for vimentin assembly.

to have an upturn in shape at low $q_{r}$ indicating absorption by a thin layer of oil on the capillary walls as demonstrated by [92]. The intensity curves for the initial measurements at positions (1) and (2), show very low or no sign of feature formation between $q_{r}$-range $0.3-0-8 \mathrm{~nm}^{-1}$. However, the subsequent measurement position hint at a developing form factor. If the time from the first point of measurement until the end of the channel is $3 \mathrm{~s}$, then effect of the $10 \mathrm{mM} \mathrm{Mg}^{2+}$ on the pre-formed filaments starts after $0.8 \mathrm{~s}$.

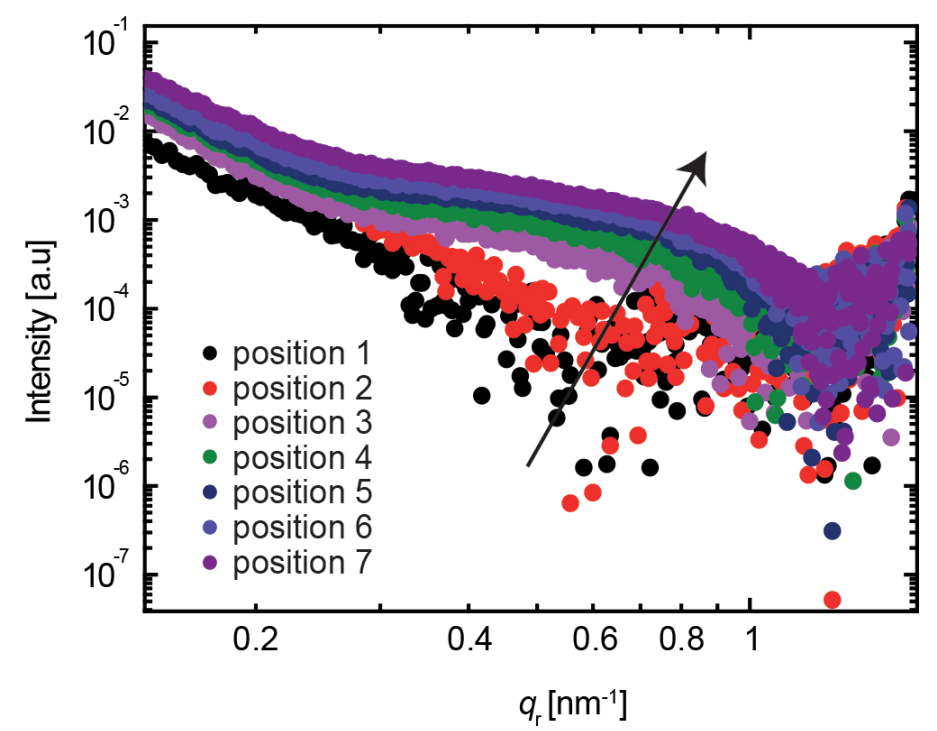

Figure 4.17: Integrated 1D-scattering intensity profiles measured at different positions on the quartz-capillary. Position 1 refers to the first droplet position after the pinch-off. The following positions are measured in chronological order. The black arrow indicates the increasing change in the feature formed in vimentin in the presence of $\mathrm{MgCl}_{2}$ with time. 


\subsubsection{Results from Measurements at AustroSAXS, ELETTRA}

Kinetics of assembled vimentin is investigated in the PDMS-Capillary devices where freshly dialyzed tetrameric vimentin with $300 \mathrm{mM} \mathrm{KCl}$ is one of the inlets (before mixing and pinch-off, final concentration in droplets is $100 \mathrm{mM} \mathrm{KCl}$ ) separated by a stream of phosphate buffer (PB) at $\mathrm{pH} 7.5$. The microfocussed beam is defined by a pinhole of $50 \mu \mathrm{m}$ to incident on the flowing droplet within the quartz capillary with an inner diameter of $80 \mu \mathrm{m}$ in order to avoid streaks arising from the capillary interfaces in the vertical direction.

The acquisition and analysis technique in this experiment differs from the previously explained "high-resolution droplet mapping" in Sec. 4.5.1. Due to the use of PILATUS 1M single-photon counting pixel detector in the present experiment, minimum sum of the acquisition, read-out and dead time between exposures is larger than the frequency of the droplet-flow. Hence, short exposures and fast acquisition is not possible. Therefore, the exposure times employed are $10 \mathrm{~min}$ utes where the scattering signal from several droplets flowing by are averaged. Background measurements are applied to empty droplets containing $\mathrm{KCl}$ and $\mathrm{PB}$ minus the vimentin prior to the actual experiment. Radially integrated intensity profiles are plotted in a double-logarithmic scale which enables us to observe very minute details in the given $q_{r}$ range extending from $0.05-1.6 \mathrm{~nm}^{-1}$. All curves are background subtracted and are corrected for transmitted intensities as shown in Fig. 4.18(a). Measurements are taken at 5 different positions along the capillary which correspond for 5 different time points and the values are given in Table. 4.1

a

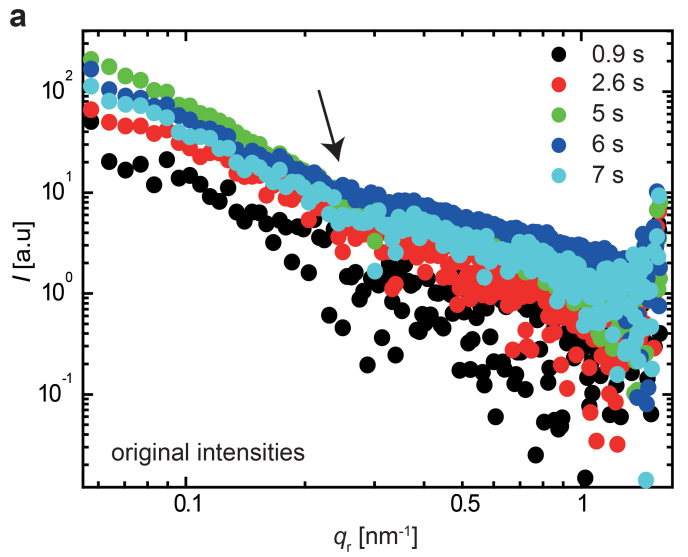

b

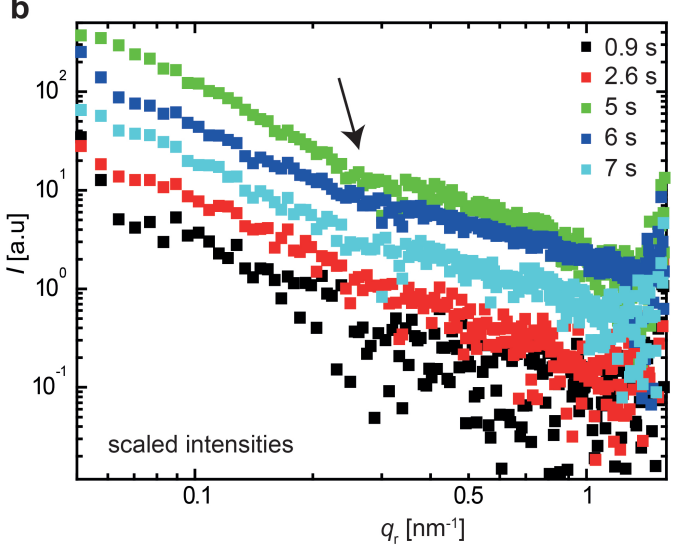

Figure 4.18.: (a) Radially integrated SAXS curves of vimentin at different stages of the assembly process. The black arrow shows the $q_{r}$ range at which there is structure formation. (b) Scaledintensity profiles of the curves from (a) for better visualization. 
Table 4.1.: Approximate time points corresponding to various stages of vimentin assembly based on droplet flowrate.

$\begin{array}{lll}\text { measurement } & \text { position on the capillary } & \text { time } \\ t_{1} & 5 \mathrm{~mm} & 0.9 \mathrm{~s} \\ t_{2} & 13 \mathrm{~mm} & 2.6 \mathrm{~s} \\ t_{3} & 25 \mathrm{~mm} & 5 \mathrm{~s} \\ t_{4} & 30 \mathrm{~mm} & 6 \mathrm{~s} \\ t_{5} & 35 \mathrm{~mm} & 7 \mathrm{~s}\end{array}$

The intensity is lowest at $t_{1}$ (the first position on the capillary (at $5 \mathrm{~mm}$ after the pinch-off which is considered the 'zero' position) at time $0.9 \mathrm{~s}$ ) which gradually increases upto time $7 \mathrm{~s}$ as the measurement progresses. The black arrow points towards a developing structure at around $q_{r}$ range of $0.28 \mathrm{~nm}^{-1}$. At this time point, vimentin tetramers have laterally assembled into so-called unit length filaments (ULFs). Thereafter, it is speculated that the elongation phase starts and a shoulder merges, hinting at more pronounced structure formation.

\subsubsection{Results from Measurements at cSAXS, SLS}

The effect of monovalent ions on the vimentin intermediate filament (IF) assembly are investigated at the cSAXS beamline. The effect of different concentrations of $\mathrm{KCl}$ on vimentin are studied in this experiment. Similar to the experiment described in Sec. 4.5.2, the composite PDMS-quartz capillary devices are utilized for the measurements where tetrameric vimentin is mixed with the $\mathrm{K}^{+}$ions separated by a stream of buffer. Three different experiments are performed to observe the effect on vimentin assembly with end concentration of $\mathrm{KCl}(50 \mathrm{mM}, 100 \mathrm{mM}, 200$ $\mathrm{mM}$ ) within the droplet after pinch-off. The flow in different channels is controlled using precise syringe pumps and flow rates are on the order of tens of microliters per hour. Similar to the measurement process described in the previous sections, different positions on the quartz capillary downstream of the pinch-off region are probed converting spatial into temporal resolution. The X-ray beam $(11.2 \mathrm{keV})$ is focused to a spot size of about $30 \times 10 \mu \mathrm{m}^{2}$ and the SAXS signal is detected in the transmission geometry at $q_{r}$ range of $0.04-1.8 \mathrm{~nm}^{-1}$.

As a control experiment, we first measure static SAXS signal in 100 micron-diameter capillaries with unassembled tetrameric vimentin and vimentin assembled in 100 $\mathrm{mM} \mathrm{KCl}$ (for 20 minutes). This is done prior to the actual measurements to check for signal characteristics as shown in Fig. 4.19. The radially integrated signal of unassembled vimentin in low salt shows a a change in the total intensity over 
$q_{r}$ which is characteristic of mono-disperse tetrameric units whereas the assembled vimentin shows a steepening at low $q_{r}$ angles which indicates the effect of $\mathrm{KCl}$ and the presence of larger particles in the scattering cross-section as shown in Fig. 4.19(a). These control experiments are complemented with quantitative measure of the particle distance distribution function where the values for the unassembled vimentin are $r$ at $2.5 \mathrm{~nm}$ and $D_{\max }$ at $12.5 \mathrm{~nm}$ which indicates tetrameric vimentin, whereas the assembled vimentin shows a larger $r$ at $7 \mathrm{~nm}$ with $D_{\max }$ at nearly $24 \mathrm{~nm}$ as shown in Fig. 4.19(b). This helps in understanding the signal characteristics in the static case which sets the basis for the timeevolution experiments.

a

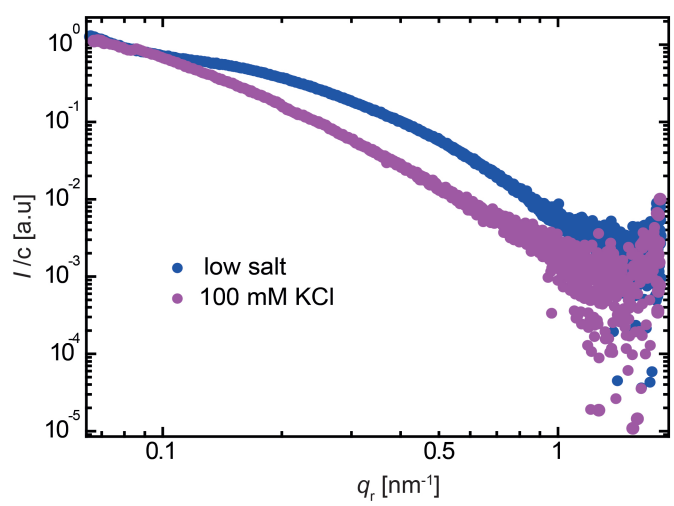

b

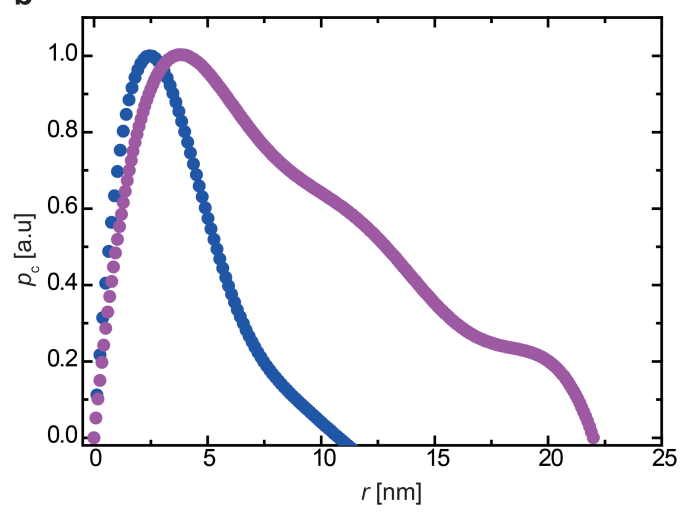

Figure 4.19.: Control experiments for vimentin signal characterization(a) Radially integrated SAXS curves of vimentin at low salt and at $100 \mathrm{mM} \mathrm{KCl}$ incubated for 20 minutes. (b) Corresponding $p_{c}(r)$ of the two curves.

For the actual droplet experiment, acquisition method involves X-ray incidence at a single position on the capillary for a exposure time of 20 minutes. The SAXS signal is averaged over several droplets flowing by during this exposure. The averaged scattered intensities are radially integrated and subtracted by a measurement of buffer-filled droplets and corrected for transmission of the beam intensity. The curves in Fig. 4.20(a), (b), (c) are plotted on a double-logarithmic scale of radial intensity $I$ versus the scattering vector $q_{r}$. Radially integrated and background subtracted curves from 6 different positions on the quartz capillary are considered for each concentration of $\mathrm{KCl}$. The color code is same for the positions in ascending order of the measurement (e.g., position1, black curve is the closest to pinch off). The insets in the figures are scaled intensities for better visualization of the de- 
veloping feature. In Fig. 4.20 , as the measurement progresses, vimentin tetramers

a

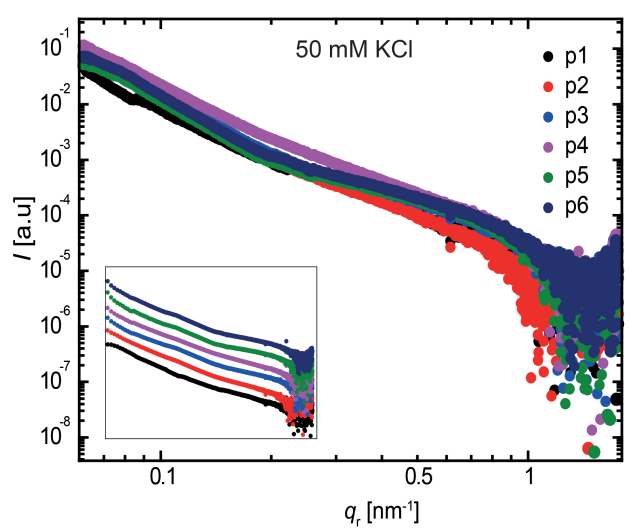

C

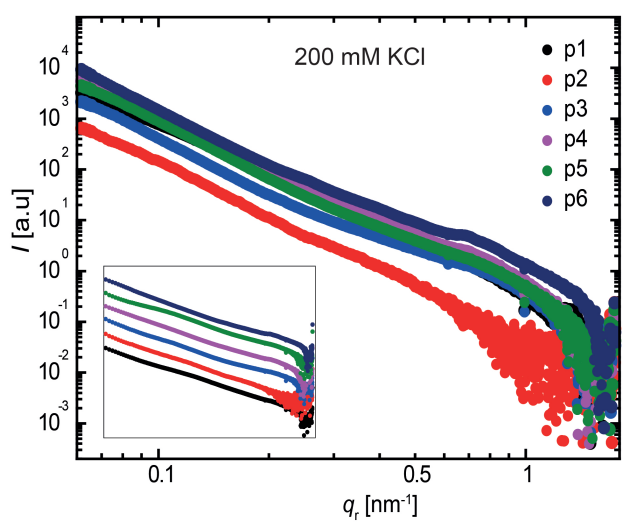

b

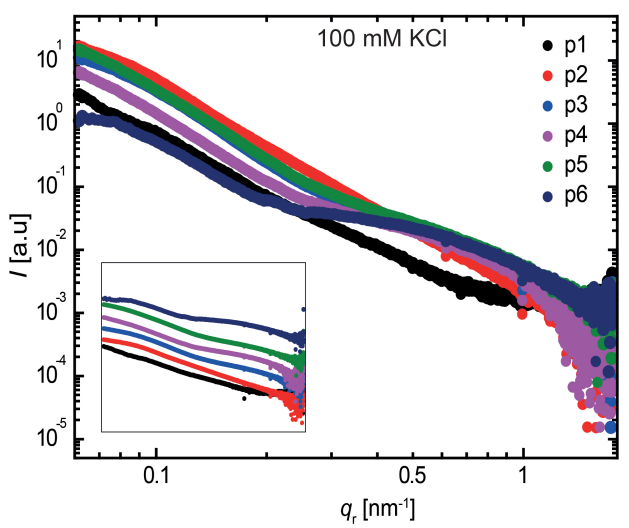

Figure 4.20.: (a) (b) (c) Radially integrated SAXS curves of vimentin at different stages of the assembly process with respect to different concentrations. Steepening is seen at lower $q_{r}$ and overlap accompanied with lower intensity is seen at higher $q_{r}$. Inset shows arbitrarily scaledintensity profiles of the curves for better visualization.

laterally assemble into so-called unit length filaments (ULFs) after which elongation starts and a shoulder at $q_{r} \approx 0.24 \mathrm{~nm}^{-1}$ emerges, hinting at more pronounced structure formation. The "shoulder" is more pronounced in the experiment with $100 \mathrm{mM} \mathrm{KCl}$ than the $50 \mathrm{mM} \mathrm{KCl}$ which can be attributed to the difference in the salt concentration. The curves from $200 \mathrm{mM} \mathrm{KCl}$ do not show any form factor formation and this could be because of higher salt which leads to faster assembly and data is smeared out. 


\subsubsection{Data Interpretation and Discussion}

Combining X-rays and microfluidics provides for a highly controllable environment and a few aspects have already been discussed in the previous section. However, several devices have been employed over the last years to make the measurement technique as efficient as possible such as the evolution of 3D-NOA-81 stickers to study protein systems with flow focusing [35, 67], NOA-Kapton device to study fixed keratin cells on $\mathrm{Si}_{3} \mathrm{~N}_{4}$ windows [97], protein crystallography studies in droplets [69] and X-ray studies on gold nano particles in flowing droplets [92]. In our studies, advantages of the composite PDMS-capillary device is the ability to build it with ease, X-ray and droplet compatibility and portability. In addition, the composite device is built with two materials: PDMS which is a standard polymeric material which enables easy method for droplet generation and the quartz capillary which is a good material for droplet flow and measurement. No additional design of the geometry is necessary such as a step to avoid protein fouling. Droplets containing several components can be produced depending on a unique geometry which can be designed in the PDMS part. The short exposure acquisition technique renders high signal-to noise ratio by thresholding the parasitic scattering signal. Hence, our methods are reliable for obtaining high resolution information and aqueous-in-oil environment make for a hassle free measurement. Not only droplets, independent particles such as flowing cells can also be flowfocused in the PDMS part and measured in the capillary region at a good signal to noise ratio.

For the experiments done at ID13, vimentin is used as a model system to test the efficiency of the device and fast acquisition accumulation technique. However, a few aspects with respect to the influence of $\mathrm{Mg}^{2+}$ ions on pre-formed vimentin filaments can be examined. It is understood from previous optical microscopy studies [52, 53], that accumulation of $\mathrm{Mg}^{2+}$ ions on the surface of vimentin filaments leading to compaction is seen only after $10 \mathrm{~s}$. Therefore, it can be safely speculated that the structure formation in our studies is an initial or intermediary stage of vimentin aggregation. Further quantitative analysis is not possible due to a non-linear Guinier region development which is common in weakly scattering biological systems. A solution to improve th quality of the measurement is to increase the contrast with a higher concentration of vimentin.

After testing the efficiency of the composite PDMS-capillary device, measurements which shed light on the vimentin assembly process are performed at ELETTRA 
and cSAXS. Measurements at ELETTRA deal with the effect of $\mathrm{K}^{+}$ions at $100 \mathrm{mM}$ concentration on vimentin assembly which have been previously studied in continuous flow experiments at several $q_{r}$ ranges in the work of Brennich et al [35] and in Sec. 4.4.1. Since the accumulation technique employed is different from the previously described short exposure acquisition, data obtained has lower signal to noise ratio which observe elongation of vimentin filaments. The evolution in the shape of the curves occur with $7 \mathrm{~s}$ which is a relevant time scale to observe assembly initiation. These measurements are further repeated with a higher beam flux at cSAXS proving a better SAXS signal.

In Fig. 4.21, curves from [35] and the cSAXS droplet datasets each have 4 curves

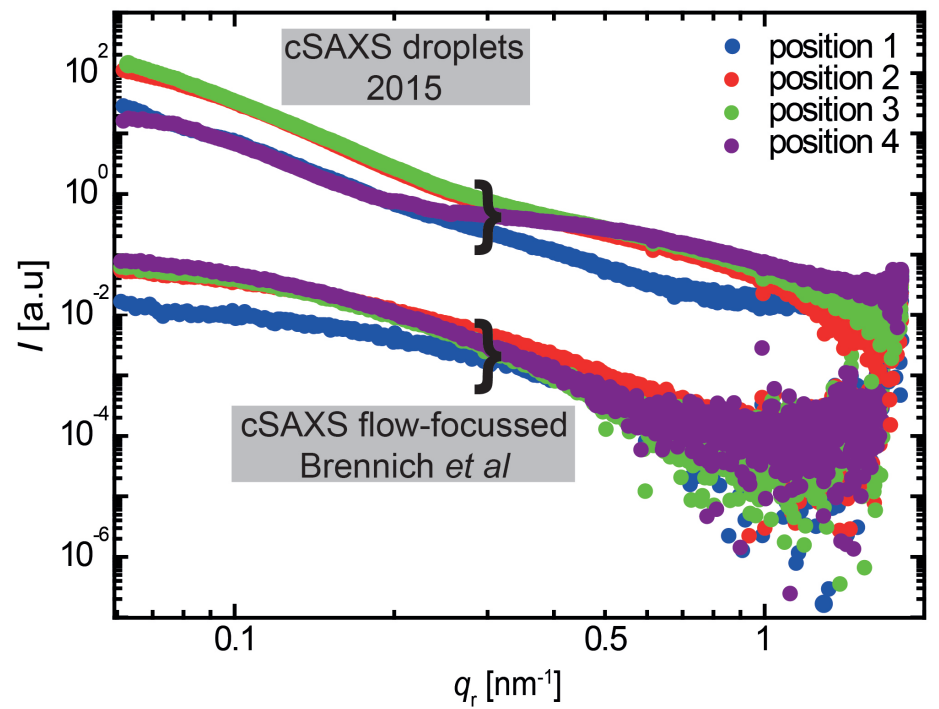

Figure 4.21: Comparison of vimentin assembly between flow focusing and droplets Radially integrated 1Dscattering intensity profiles measured at different positions on the quartz-capillary are compared to different positions in the flow focused stream [35]. The following positions are measured in chronological order. More feature formation is seen in droplet data.

for comparison. The curves from flow focused experiment are datapoints measured along the flow and the legend shows curves in ascending order of the positions (vimentin assembly in $200 \mathrm{mM} \mathrm{KCl}$ ). The same colors are used for the droplets to show data measured in the ascending order from the pinch off (e.g., position 1, blue curve is closest to pinch off and vimentin in droplets assembled in $100 \mathrm{mM} \mathrm{KCl}$ ). Both datasets have a comparable $q_{r}$ range, which makes it easier to see the similarities and differences. Droplet curves are steeper at lower $q_{r}$ compared to the flow focused curves (the Guinier region which gives us the size parameter). This could be because the signal to noise ratio is poor in droplets owing to the influence of oil in the droplet experiment and since the scattering signal is averaged over several droplets. A "shoulder" develops in the droplet curves 
as measurement progresses. Since every droplet contains the same concentration of $\mathrm{KCl}$ and vimentin, mixing could be faster and hence the form factor develops sooner than in the flow focused experiment. The concept of a pico-liter sized droplet being an unique in-flow microreactor is similar to the reactions occurring in stationary capillaries. This allows us to quantitatively compare the shape of the radially integrated curves for vimentin filament assembly.

a

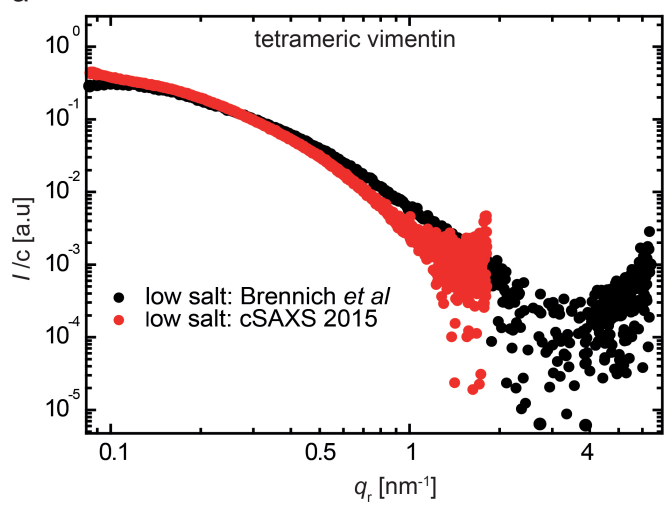

$b$

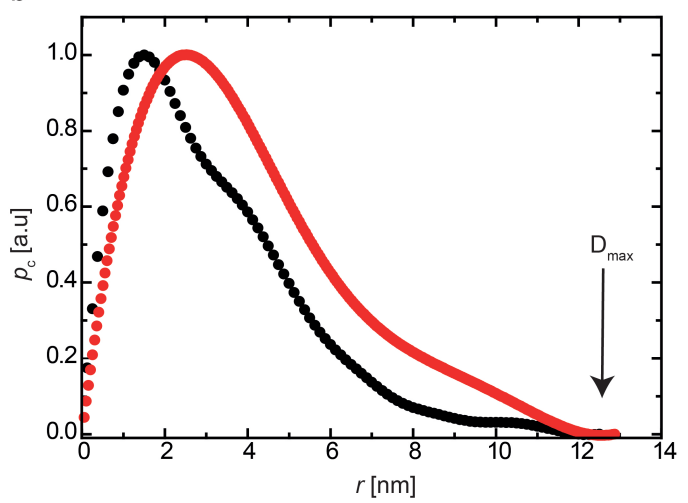

Figure 4.22.: (a) Radially integrated SAXS curves of unassembled vimentin from cSAXS 2015 and from [37]. (b) corresponding $p_{c}(r)$ of the two datasets

To characterize the quality of the scattering signal, control-experiments of unassembled vimentin at low salt (in 100 micron-diameter) is compared to the work of Brennich et at. [37]. The general shape of the two curves are similar especially at lower $q_{r}$ range and the $D_{\max }$ values are comparable as shown in Fig. 4.22 . A $r$ value difference is observed between the two measurements owing to the difference in the tetrameteric population found in the respective sample. When comparing vimentin assembled in $100 \mathrm{mM} \mathrm{KCl}$ in Fig. 4.23, we observe that the angle of steepening at lower $q_{r}$ range is similar in the two datasets but the development of the kinks is more pronounced in the work of Brennich et al. This is also justified in the $D_{\max }$ values in Fig. 4.23(b), since vimentin is assembled in epicups for 15 hours and incubated in the capillaries before measurement, whereas the control experiment at cSAXS has a limited assembly time of 20 minutes. From this comparison and from the data presented in Fig. 4.20, we can opine that the length scales accessible with droplets have a particle diameter of $\approx 20-25 \mathrm{~nm}$ in real space with an assortment of various sized-populations seen from the shape of the PDDFs. The time accessible is upto $7 \mathrm{~s}$ from the time of droplet pinch-off which is sufficient to probe the initial kinetics of vimentin assembly. 


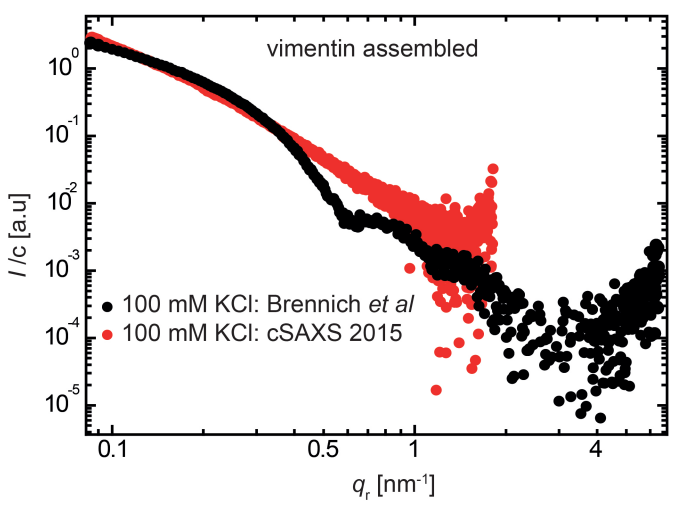

b

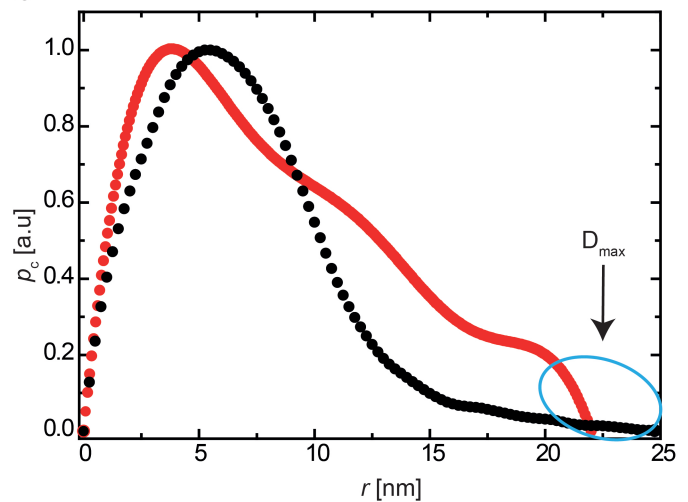

Figure 4.23.: (a) Radially integrated SAXS curves of assembled vimentin from cSAXS 2015 and from [37]. (b) corresponding $p_{\mathcal{C}}(r)$ of the two datasets

In conclusion from the studies of droplets, we demonstrate the development of a state-of-the-art combination of X-ray/droplet compatible microfluidic device and high-resolution measurement technique to study biological processes in flow and in particular the effect of mono and divalent ions on the nanostructure of vimentin. The advantage in droplet experiments lies in the fact that even a highly adherent protein like vimentin can be studied by an emulsion confinement.

\section{New Challenges in Protein Kinetics with Emulsion Science}

A combination of X-ray scattering and continuously flowing protein samples is a powerful technique to understand the kinetics induced due to factors such as addition of ions, changes in $\mathrm{pH}$, concentrations, temperature etc. Additionally, studies extending from one-phase flow to a multi-phase phase flow are emerging. A variety of bio-molecular process such as collagen assembly [98], DNA condensation [99], RNA folding [72] and vimentin assembly [35] have been studied with the continuous flow-focusing technique in the past. The use of continuously flowing sample through the area of the exposed X-rays prevents radiation induced artifacts since the sample flowing is continuously renewed. Also, measurements at different positions converts spatial informations into temporal information by probing positions downstream in the channel following the mixing and reaction times. However, tracking kinetics in proteins can be very tricky due to protein adhesion to the surface in a one-phase flow setup. A suitable technique to circumvent the limitations posed by one-phase microfluidics is droplet based multi-phase microfluidics. Droplets serve as nano-liter vessels that compartmentalize the flow- 
ing samples by using small sample detection volumes. But new challenges emerge when combining X-ray scattering and emulsions which are documented in 3 reports so far: the Ismagilov group makes use of a composite microfluidic system of polydimethylsiloxane (PDMS) and glass microcapillary to perform protein crystallization in aqueous droplets. The reactants are combined in the PDMS microchannel and then emulsified to form droplets which then are collected in the capillary and probed by in situ X-ray diffraction [69]. But this approach does not exploit the advantages of SAXS analyses in continuous microfluidic flow. The Pfohl group study the liquid crystal $8 \mathrm{CB}$ to form drops in water probed by SAXS in the microchannel [100]. The Seiffert group focuses on obtaining quantitative data from flowing droplets with SAXS which compartmentalize gold nanoparticles as test material [92]. Apart from these 3 papers, no work to the best of my knowledge has yet been published which takes advantage of this promising approach of flowing droplets and $\mathrm{X}$-ray scattering with low sample consumption.

In the domain of this thesis, we present a combination of droplet-based microfluidics technique with SAXS detection to establish a methodology which can be extended to various biological process to obtain quantitative results with a high signal-to-noise ratio. The main advantage of such a system is the utilization of small drop volume and low sample consumption, since the sample is made to continuously flow within droplets through the beam. However, it helps to understand the nuances of the scattering signal from the small sample volume since the scattering power of an object highly correlates to its irradiated volume of the sample. Typically, the higher the electron density (i.e., higher sample volume), the more waves scatter. If the sample is just one particle of volume $V_{1}$ with an electron density of $\rho_{1}$, then $V_{1} \rho_{1}$ wave amplitudes are scattered. The intensity (i.e., detector read-out) is the square of all wave amplitudes that come from this volume. Therefore, the total scattered intensity of this particle $I_{1}(q)$ is [94, 101]:

$$
I_{1}(q)=I_{0} \cdot \rho_{1}^{2} \cdot V_{1}^{2} \cdot X
$$

where $X$ stands for the inter- and intra-particle interference factors. However, in our approach the scattering from the small sample volume can be compensated by the detection method. Here the short exposure times "map" each flowing droplet giving us a high resolution in a localized real space. Thus the crux of this technique lies in the fact that emulsion science offers several possibilities for studying protein kinetics where small volumes can be effectively controlled and 
tracked at high-resolution. One may find the applications of this approach to extend to studying encapsulated proteins or even other biological substances such as red blood cells by using 3rd-generation synchrotron sources or X-ray free-electron lasers [102, 103].

\subsection{Summary and Closing Remarks}

In this chapter, we have confirmed that SAXS is a suitable technique to probe structures on the order of nanometers. Since it is inherently a "slow" technique, we combine it with microflow to gain a high temporal resolution with respect to vimentin assembly kinetics. For this purpose, we adapt two different microfluidic approaches:

- In the continuous-flow experiments, the serial-inlet device geometry lets us add the ions in a subsequent manner. We can access the surface sensitive Porod region of the scattered intensities of the assembling vimentin. Hence, observations of the simultaneous smoothing and compaction of these intermediate vimentin assemblies are made on the order of seconds.

- In the droplet experiments, prevalent challenges such a clogging seen in onephase flow are eliminated. Measurement of the droplets containing vimentin and monovalent salt is done in two ways: a fast acquisition method with shorter exposures and a longer acquisition method with constant exposure. Of the two, we prefer the fast acquisition method due to a higher signalto-noise ratio in the measurements. We have, thus, shown a high-spatial resolution state-of the-art measurement technique to obtain information of flowing droplets, for which vimentin acts as a model system.

\section{Insights into Concentration Variability for Assembly Process}

The interactions underlying the self-assembly process of vimentin can be understood broadly based on two different approaches:

- a static scenario where the volume and the initial concentration of both the protein and ions remain constant and snap-shots of the interacting particles can be obtained to give us information of the assembly and elongation process. 
- a dynamic scenario where the concentration of protein and ions changes with time, which takes into consideration the attractive and repulsive forces governing the interactions.

Solution SAXS in capillaries and light scattering experiments can confirm the salt concentration independence (within the sample volume) on the self-assembly process of the first case. However, the continuous flow experiments with SAXS renders a concentration dependent scattering which will likely lead to either interparticle interference or aggregation effects if a critical concentration is not implied [104]. Therefore, by employing time-resolved SAXS, we reach a minimal critical concentration of the monovalent ions which is necessary to unblock the formerly unproductive head domains in the vimentin tetramers and thus initiate assembly as discussed in detail in Sec. 4.4.1

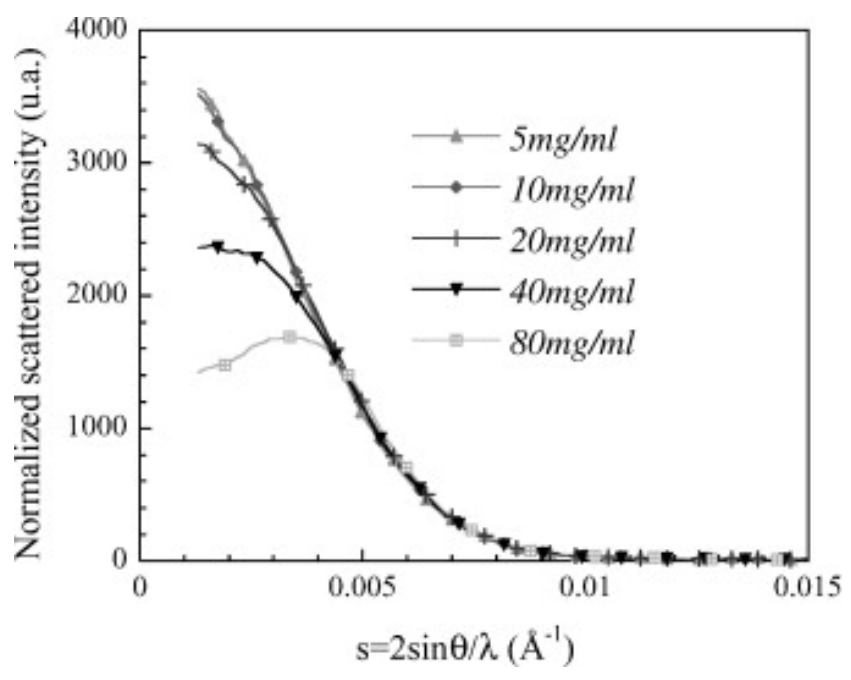

Figure 4.24.: Demonstration of scattered intensity variation as a function of protein concentration. $\alpha$-crystallin solutions as a function of protein concentration are shown, at $20^{\circ} \mathrm{C}$, in $150 \mathrm{mM}$ phosphate buffer at $\mathrm{pH}$ 6.8. An increase of protein concentration induces a decrease of the scattered intensity and of the structure factor at low angles, which is characteristic of repulsive interactions. Graph is adapted from [105].

In a scattering experiment, concentration effects become visible at small angles where a descent in the intensity at small angles is typical for repulsive interaction potentials and a intensity increase indicates attractive interactions signifying selfaggregation processes. An excerpt of the variation in concentration for $\alpha$-crystallin, an eye lens protein is shown for demonstration of the interactions in Fig. 4.24 . With these conditions into considerations, we explore the concentration variability 
of vimentin in the assembly process with static light scattering (SLS) in Chap. 5 , where the initial concentrations are very dilute in comparison to the initial concentration used in SAXS experiments. To account for the interactions seen between vimentin and the ions at low protein concentrations with SLS, Zimm analysis is performed as explained in Sec. 5.1.1. There the intensity is extrapolated to zero scattering vector angle ('zero'- $q$ ) and zero concentration yielding parameters such as the molecular weight and the radius of gyration (size parameter of the assembling particles). 



\section{Time-resolved Light Scattering for Vimentin Kinetics}

Oh leave the Wise our measures to collate.

One thing at least is certain, light has weight.

One thing is certain and the rest debate.

Light rays, when near the Sun, do not go straight.

Sir Arthur Eddington, 1919

Impact of ion valency on the assembly of vimentin is previously studied by a combination of microfluidics and SAXS [35] and solution SAXS for structural details of assembling intermediates [37, 106] to elucidate sizes upto $\approx 16-25 \mathrm{~nm}$ diameter of the filaments. Quantitative imaging of the vimentin filaments in the advanced phase of the assembly process with structures that are several $\mu \mathrm{m}$ long are studied with optical microscopy techniques [33, 42]. Influence of divalent ions on the network formation of vimentin filaments in droplets which are of the order 


\section{\begin{tabular}{l|l} 
Chapter $\mathbf{5}$ & TIME-RESOLVED LIGHT SCATTERING FOR VIMENTIN KINETICS
\end{tabular}}

of several hundred $\mu \mathrm{m}$ have been studied with florescence imaging [52, 53, 54]. However, information of the intermediate length scales that follow vimentin filament elongation (inaccessible by both $\mathrm{X}$-rays and optical microscopy) has been missing. Therefore, light scattering experiments on vimentin are performed as a bridging technique to access the longitudinal assembly of vimentin IFs by analyzing the time-dependent length distribution.

In this chapter, time resolved multi-angle combined dynamic light scattering (DLS) and static light scattering (SLS) experiment is performed to evaluate the assembly and elongation process of vimentin due to the influence of $\mathrm{KCl}$. Averaged values for the radius of gyration $R_{g}$ and the hydrodynamically effective radius together with the mass values of the developing vimentin filaments are elucidated. A brief theory of SLS and DLS is explained in Sec. 5.1 followed by the experimental procedure for the measurements in Sec. 5.2 where focus is on the instrument that performs time resolved simultaneous SLS and DLS. The instrument is utilized for a simultaneous recording of the time resolved evolution of the kinetics of vimentin assembly. This enables to follow growth processes with a time resolution of 'seconds to minutes'. The simultaneous time resolved technique was first applied to the investigation of aggregation process of azo dyestuff mixture in the presence of $\mathrm{Mg}^{2+}$ in a custom-developed multi-angle scattering instrument [107]. Subsequently, the results of the time resolved tracking process and a kinetic model for filament growth are proposed in Sec. 5.3 .

Parts of this chapter (Sec. 5.2 and Sec.5.3 are a preliminary version for a manuscript which was initially prepared. It was iteratively written and improved over several versions and later published in Proceedings of the National Academy of Sciences (PNAS, USA) [108]. Author contributions are as follows: Carlos G.Lopez and Oliva Saldanha are co-first authors and contributed equally to the work. Klaus $\mathrm{Hu}-$ ber and Sarah Köster designed the research; Carlos G.Lopez and Oliva Saldanha. performed research; Carlos G.Lopez analyzed data and performed theoretical calculations; Carlos G.Lopez, Oliva Saldanha, Klaus Huber, and Sarah Köster wrote the paper; Oliva Saldanha prepared protein samples; Klaus Huber interpreted data and performed theoretical calculations; and Sarah Köster interpreted results. 


\subsection{Theory}

\subsubsection{Static Light Scattering}

The basic principles governing static light scattering are analogous to X-ray scattering which is discussed in Sec. 4.1. Both processes are based on elastic scattering of electromagnetic radiation (Rayleigh scattering), where the energy (and therefore the wavelength) of the incident photon is conserved and only the direction of the scattered photon changes. In this case, the scattering intensity is proportional to the fourth power of the reciprocal wavelength of the incident photon. As shown in Fig. 4.1 from Sec. 4.1 , the scattering vector $\vec{q}=\vec{k}^{\prime}-\vec{k}$ varies with both the scattering angle and the wavelength. Therefore, in a typical scattering experiment, be it SLS or X-ray, the angle $2 \theta$ or $\lambda$ or both can be varied to observe the scattered intensity in the relevant $q$-range. Since the process occurring is elastic, the wave vectors of incident and scattered waves are equal $|\vec{k}|=\left|\overrightarrow{k^{\prime}}\right|$. From the cosine rule,

$$
\begin{aligned}
q^{2} & =k^{2}+k^{\prime 2}-2 k k^{\prime} \cos \theta \\
& =2 k^{2}(1-\cos \theta) \\
& =2 k\left[2 \sin ^{2}\left(\frac{\theta}{2}\right)\right] \\
& =\frac{16 \pi^{2}}{\lambda^{2}} \sin ^{2}\left(\frac{\theta}{2}\right) \\
q & =\frac{4 \pi}{\lambda} \sin \left(\frac{\theta}{2}\right)
\end{aligned}
$$

From Bragg's law, we get $n \lambda=2 d \sin \left(\frac{\theta}{2}\right)$, where $d$ is the distance between two atoms. For $n=1$, the length scales accessible in " $q$ " - space is given by $q \approx \frac{2 \theta}{d}$. An example of the real space distances accessible by light and X-rays are shown in Tab 5.1.

Table 5.1.: Accessible real-space length scales

\begin{tabular}{lll}
\hline & $q$-range $\left(\mathrm{nm}^{-1}\right)$ & distance $d$ \\
\hline visible light & $10^{-4}-0.05$ & $0.1 \mu \mathrm{m}-60 \mu \mathrm{m}$ \\
X-rays & $0.002-10$ & $1 \mathrm{~nm}-30000 \mathrm{~nm}$ \\
\hline
\end{tabular}

In a typical light scattering experiment, the measured scattered intensity for a 


\section{\begin{tabular}{l|l} 
Chapter 5 & TIME-RESOLVED LIGHT SCATTERING FOR VIMENTIN KINETICS
\end{tabular}}

system of small interacting particles is given by:

$$
I(\vec{q})=c(\Delta \rho)^{2} V^{2} P(\vec{q}) S(\vec{q})
$$

where $c$ is the concentration of the particles in solution, $\Delta \rho$ is the difference in electron densities, $\mathrm{V}_{p}$ is the particle volume, $P(\vec{q})$ is the form factor of a single particle which determines the shape and size of that particle, $S(\vec{q})$ is the structure factor which gives arrangement of particles in solution and in the case dilute dispersion with non-interacting particles, we assume $S(\vec{q})=1$. Here, we bring to attention that the Eqn. 5.2 is the same as Eqn. 4.7, which was previously explained to understand the scattering by X-rays.

The Rayleigh-Gans-Debye (RGD) theory is the most common theory used to describe light scattering. This theory assumes that light is not attenuated with the particle (adsorption) nor is it reflected at the medium-particle boundary. This occurs if the following conditions are true:

$$
\begin{aligned}
|1-m| & \ll 1 \\
\frac{2 \pi n_{s}}{\lambda} a|1-m| & \ll 1
\end{aligned}
$$

where $\lambda$ is the wavelength of light, $m=\frac{n_{p}}{n_{s}}$ is the relative refractive index (R.I.) i.e., the ratio of the R.I. of the interacting particles to the suspension medium and $a$ is the characteristic size of the small objects.

Two conditions occur within this assumption

- Each particle scatters as if isolated or unperturbed from the other particles

- Each particle is subjected to the same electromagnetic radiation of the incident light (no effect of absorption is taken into account)

In such a situation, the RGD assumption enables us to write the scattered intensity collected at the angle, $\theta$ at a distance $R_{0}$ from the sample as:

$$
I(q, c)=I_{i} \frac{f(\theta)}{R_{0}^{2}} c V_{s} \Delta \rho^{2} V_{p}^{2} P(q)
$$

which is a variant of Eqn. 5.2. Here $I_{i}$ is the incident intensity, $f(\theta)$ is the geometrical factor accounting for the polarization of incident and collected light, $V_{p}$ and $V_{s}$ are the particle and scattering volumes. Only $P(q)$ remains since we assume $S(q)=1$. 
A typical application of the scattering intensities from a protein or a polymer is the determination of the weight averaged molecular weight of the solute $M_{w}$. Simultaneously, by measuring the scattering intensity at different angles the root mean square radius called the radius of gyration $R_{g}$ can be determined and by measuring the scattered intensity for one macromolecule at various concentrations gives the second virial coefficient $A_{2}$.

The most common method to approximate the scattered intensities from a dilute solution of weakly interacting particles is given by the Zimm equation:

$$
\frac{K_{c}}{R_{\theta}}=\frac{1}{P(q) M_{w}}+2 A_{2} C \simeq \frac{1}{M_{w}}\left(1+\frac{q^{2}\left\langle R_{g}{ }^{2}\right\rangle_{z}}{3}\right)+2 A_{2} C
$$

where $R_{\theta}$ is the excess Rayleigh ratio, $c$ is the concentration in $\mathrm{g} / \mathrm{L}, \mathrm{K}$ is a constant given by $K=\frac{4 \pi^{2} n_{s}^{2}}{N_{A} \lambda^{4}}\left(\frac{d n}{d c}\right)^{2}, N_{A}$ is Avagadro's number, $n_{s}$ is the R.I of the solvent, $\left(\frac{d n}{d c}\right)$ is the R.I increment of the solute in the solvent, $q$ is the scattering vector, $\lambda$ is the wavelength of the laser, $\theta$ is the scattering angle, $M_{w}$ is the weight averaged molecular weight of the solute, $\left\langle R_{g}{ }^{2}\right\rangle$ is the z-averaged square radius of gyration, $A_{2}$ the second virial coefficient and $P(q)$ is the form factor of the solute particle.

The approximation $P(q) \simeq 1+\frac{q^{2}\left\langle R_{g}{ }^{2}\right\rangle_{z}}{3}$ holds when $q R_{g} \leq 1$, where $R_{g}=\sqrt{\left\langle R_{g}{ }^{2}\right\rangle_{z}}$ [109, 110].

The form factor for thin rods of length $L$ is given by [111, 112]:

$$
P(q, L)=\frac{2}{q L} \int_{0}^{\infty} \sin (\mathrm{q} L) /(q L) d L-\left(\frac{\sin (q L / 2)}{q L / 2}\right)^{2}
$$

For polydisperse systems, the form factor $P_{z}(q)$ is the z-average over a distribution of particle sizes:

$$
P_{z}(q, L)=\frac{\int_{0}^{\infty} w(L) L P(q, L) d L}{\int_{0}^{\infty} w(L) L d L}
$$

$w(L)$ is the weight distribution function of particle length $L$, which can be modeled by a Schultz-Zimm (SZ) function, based on the weight average of the length $L_{w}$ [109]:

$$
w(L)=\left(\frac{z+1}{L_{w}}\right)^{z+1} \frac{L^{z}}{\Gamma(z+1)} \exp \left(-\frac{(z+1) L}{L_{w}}\right)
$$

where $\Gamma$ is the Gamma function. The z-average of the squared radius of gyration $\left\langle R_{g}{ }^{2}\right\rangle_{z}$ for polydisperse thin rods following a SZ distribution is given by [113]: 


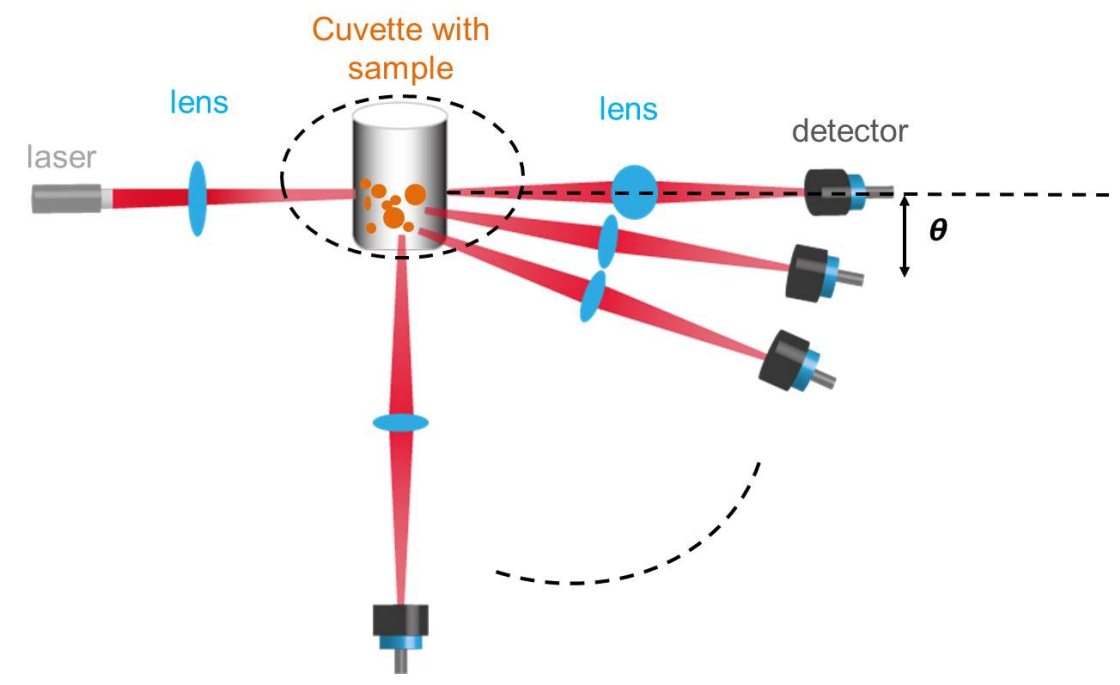

Figure 5.1.: Schematic Static Light Scattering Image. A laser is illuminated on a cuvette containing the sample to be analyzed. One or many detectors are used to measure the scattering intensity in dependence of the scattering angle $\theta$.

$$
\left\langle R_{g}{ }^{2}\right\rangle_{z}=\frac{(z+3)(z+2)}{(z+1)^{2}} \frac{L_{w}{ }^{2}}{12}
$$

The first to third moments of the molar mass for a SZ distribution are related by:

$$
\text { polydispersity }(p . d .)=\frac{M_{w}}{M_{n}}=\frac{z+1}{z} ; \frac{M_{z}}{M_{w}}=\frac{z+2}{z+1}
$$

The second virial coefficient for cylinders of diameter $d$ is approximated by [114]:

$$
A_{2}=\frac{N_{A} \pi d^{2} L}{4 M^{2}}\left(1+\frac{L}{d}\left(1+\frac{d}{2 L}\right)\left(1+\frac{\pi d}{2 L}\right)\right)
$$

\subsubsection{Dynamic Light Scattering}

Dynamic light scattering (DLS) (also known as Photon Correlation Spectroscopy or Quasi-Elastic Light Scattering) is an important light scattering technique that is useful in determining particle sizes of molecules upto $1 \mathrm{~nm}$ diameter. Typical applications are micelles, emulsions, polymers, nanoparticles, colloids, polymers [115] and more recently in organic studies such as proteins in food science [116, 117]. The basic principle involves the sample being illuminated by a laser beam and the fluctuations of the scattered light detected at a known scattering angle $\theta$ by a fast photon detector. DLS measures these time-dependent fluctuations 
(occurring due to Brownian motion of the molecules in solution) in the scattering intensity to determine the translational diffusion coefficient $D$ (also adapted at $D_{z}$ in the present scenario which is the $z$-averaged diffusion coefficient) and subsequently the hydrodynamic radius $R_{H}$ where the rate of the intensity fluctuations is dependent on the size of the particles. Therefore, it is critical to have a solution devoid of dust or artifacts from the solution which can be removed by filtration or centrifugation.

The measured data in a DLS experiment is the autocorrelation curve as given in Eqn. 5.12. Embodied within this correlation curve is all of the information regarding the diffusion of particles within the sample being measured. The second order autocorrelation curve is generated from the intensity trace by:

$$
g_{2}(t, q)=\frac{\left\langle I\left(t_{0}\right) I\left(t_{0}+t\right)\right\rangle}{\left\langle I\left(t_{0}\right)\right\rangle^{2}}
$$

where $g_{2}(t, q)$ is the autocorrelation function at a particular wave vector, $q$, and delay time, $t$, and $I$ is the intensity. The diffusion coefficient $D$ or $D_{z}$ is our case, is calculated by fitting the correlation curve to an exponential function, with $D_{z}$ being proportional to the lifetime of the exponential decay. $R_{H}$ is then calculated from the diffusion coefficient using the Stokes-Einstein equation, where $k_{B}$ is the Boltzmann constant, $T$ is the temperature, $\eta$ is the medium viscosity, and $f=$ $6 \pi \eta R_{H}$ is the frictional coefficient for a hard sphere in a viscous medium and is shown by:

$$
D_{z}=\frac{k_{B} T}{f}=\frac{k_{B} T}{6 \pi \eta R_{H}}
$$

The second-order intensity autocorrelation function can be related to the first order field autocorrelation function by Siegert's equation as follows:

$$
g_{2}(t, q)=1+\beta\left[g_{1}(t, q)\right]^{2}
$$

where $\beta$ is a constant dependent on the geometry and focus of the laser source. For a monodisperse population of the solute, the first-order autocorrelation is treated as a single exponential decay by:

$$
g_{1}(t, q)=e^{-\Gamma t}
$$

where $\Gamma$ is the decay time. Generally, a distribution $(G(\Gamma))$ of decay times is 


\section{\begin{tabular}{l|l} 
Chapter $\mathbf{5}$ & TIME-RESOLVED LIGHT SCATTERING FOR VIMENTIN KINETICS
\end{tabular}}

expected for a polydisperse system:

$$
g_{1}(t, q)=\int G(\Gamma) e^{\Gamma t} d \Gamma
$$

A simple approach [118, 119, 120] to extract the moments of the distribution is by a modified cumulant method, where $g_{1}(t, q)$ is expanded as:

$$
g_{1}(t, q)=e^{\Gamma t}\left(1+\frac{\mu_{2} t^{2}}{2 !}-\frac{\mu_{3} t^{3}}{3 !}+\ldots\right)
$$

with $\Gamma, \mu_{2}$ and $\mu_{3}$ the first, second and third cumulants respectively. Due to limitations in data quality, it is common to fit $g_{1}(t, q)$ with only the first two cumulants. In dilute solution, $\Gamma$ depends on the scattering vector in the low $q$ limit as:

$$
\frac{\Gamma}{q^{2}}=D_{z}\left(1+q^{2}\left\langle R_{g}^{2}\right\rangle_{z} C+k_{D} c\right)
$$

$C$ is a constant and $D_{z}$ is the $z$-averaged diffusion coefficient and $k_{D}$ accounts for the concentration dependence of $\Gamma$.

The first cumulant $\Gamma$ is used to calculate the z-average diffusion coefficient $D_{z}$ via Eqn. 5.18. The second cumulant $\mu_{2}$ in Eqn. 5.17 is the variance $\left(\left\langle\Gamma^{2}\right\rangle_{z}-\Gamma_{z}^{2}\right)$ of $\Gamma$. It can be used to calculate the polydispersity p.d. $=M_{w} / M_{n}=1+1 / z$ of rod like molecules [121]:

$$
\frac{M_{w}}{M_{n}}=1+\frac{\mu_{2}}{\Gamma^{2}}
$$

The $z$-averaged diffusion coefficient for a polydisperse cylinders, where the length distribution follows the Schulz-Zimm formula given by [122]:

$$
D_{z}=\frac{k_{B} T}{3 \pi \eta L_{w}}\left(1+\sqrt{\frac{6}{\pi}}\left(\Psi(1+z)-1-\ln \left(\frac{(z+1) d}{L_{w}}\right)\right)\right.
$$

where $d$ is the cylinder's diameter and $\Psi$ is the digamma function. 


\subsection{Experimental Section}

\subsubsection{Determination of Protein Concentration}

Concentration of dialyzed tertrameric vimentin in solution is determined by NanoDrop ND-1000 spectrophotometer (NanoDrop ${ }^{\text {TM }}$ Technologies, Wilmington, USA) which is based on UV-spectra recorded at a wavelength of $280 \mathrm{~nm}$ as explained in Sec. 2.4.2 $2 \mu \mathrm{L}$ protein volume per reading is sufficient for the measurements. Nominally, the mean of atleast three readings are performed to determine the final protein concentration. The concentration of several protein samples $(\approx 20$ samples) is obtained before and after the filtering. The filtering procedure is explained in Sec. 5.2.2. Both the initial and final concentration values do not show any effect with regard to filtering. Hence, it is concluded that absorption of protein while filtering is non-existent.

\subsubsection{Preparation for Light Scattering Experiments}

Cylindrical quartz cuvettes from Helma with a diameter of $20 \mathrm{~mm}$ are used as scattering cells. Prior to any measurement, cuvettes are cleaned from dust by continuously injecting freshly distilled acetone from below for $5 \mathrm{~min}$. All aqueous solutions (phosphate buffer, $\mathrm{KCl}$ and tetrameric vimentin in solution) are filtered with $0.22 \mu \mathrm{m}$ cellulose ester filters before each measurement. These filters are first washed with the appropriate solvents before use. In order to track the kinetics of assembly and elongation of vimentin, $3 \mathrm{~mL}$ of vimentin solution is filtered into a cuvette followed by $3 \mathrm{~mL}$ of the $\mathrm{KCl}$ which is dissolved in $2 \mathrm{mM}$ phosphate buffer. The cuvette is shaken to promote mixing and placed into the slot for measurements in the light scattering instrument. A dead time of $15-30 \mathrm{~s}$ is recorded for several samples before the actual reading is taken.

\subsubsection{Combined Setup for Time-Resolved SLS and DLS}

An ALV/CGS-3/MD-8 Multidetection Laser Light Scattering Goniometer System simultaneously analyzes static and dynamic light scattering at 8 different observation angles (see Fig. 5.2). The angle between two neighboring detectors is $8^{\circ}$. A laser at a wavelength of $632.8 \mathrm{~nm}$ and a power of $35 \mathrm{~mW}$ (HeNe laser, Soliton Laser- und Messtechnik GmbH, Giching, Germany) is used as a light source. The full accessible regime of momentum transfer is $0.0046 \mathrm{~nm}^{-1}<q<0.024 \mathrm{~nm}^{-1}$, depending on the solvent. The array of detectors as a whole can be freely rotated 


\section{\begin{tabular}{l|l} 
Chapter 5 & TIME-RESOLVED LIGHT SCATTERING FOR VIMENTIN KINETICS
\end{tabular}}

around the goniometer between a minimum scattering angle of $20^{\circ}$ for the left most detector and a maximum scattering angle of $136^{\circ}$ for the right most detector. Each of the eight detectors contains an avalanche photodiode along with a photon correlator. In this way, the static scattering intensity and the intensity correlation can be recorded simultaneously over an angular range of $56^{\circ}$, which allows for the extrapolation of both the SLS and DLS results to zero scattering angle [107]. A second combined SLS/DLS instrument (see Fig. 5.3) with two detectors at a single angle is employed for samples which are not time-dependent (prior to the addition of salt) or for measurements with slow kinetics such that no significant change occurred in the typical acquisition time for a scattering curve ( $\approx 5 \mathrm{~min}$ ).

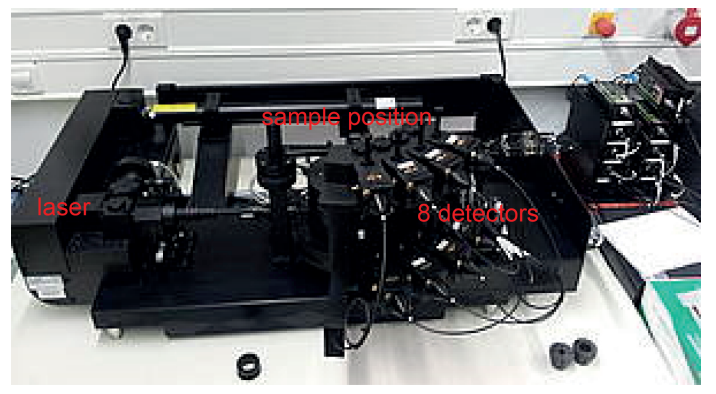

Figure 5.2.: Photograph of the 8-angled light scattering instrument. The ALV/CGS-3/MD8 Multidetection Laser Light Scattering Goniometer System simultaneously analyzes static and dynamic light scattering at 8 different observation angles.

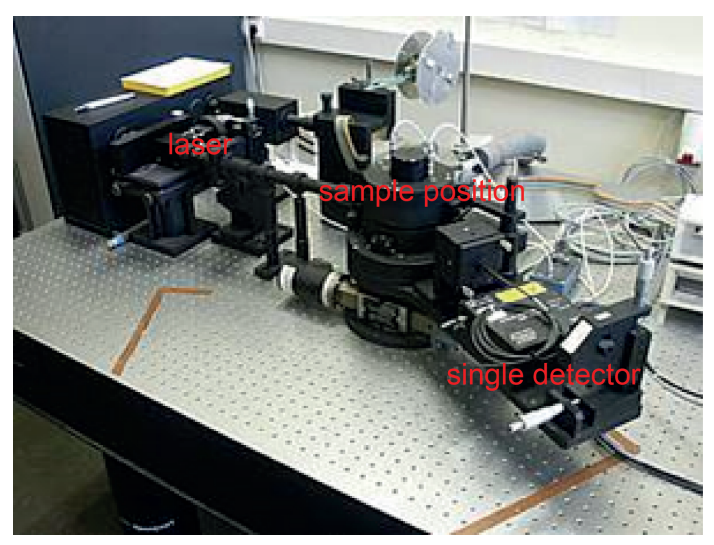

Figure 5.3.: Photograph of the single-angled detection light scattering instrument. The ALV 5000E Compact Goniometer System (ALV 5000E CGS) simultaneously analyzes static and dynamic light scattering at variable observation angles, single angle at a time. A laser at a wavelength of $632.8 \mathrm{~nm}$ and a power of $35 \mathrm{~mW}$ (Soliton HeNe) is used as a light source. 


\subsubsection{Treatment of Scattering Data}

Absolute calibration of the measurements is done against toluene. The recorded scattering intensity of the buffers ( $\mathrm{KCl}$ in $2 \mathrm{mM}$ phosphate buffer) is subtracted from that of the sample. For kinetic readings, where only 8 angles are available, $R_{g}, M_{w}, D_{z}$ and $C$ were obtained from linear regression of equations from Sec. 5.1 setting $A_{2}$ and $k_{D}=0 . M_{w} / M_{n}$ was obtained from least squares fitting of Eqn. 5.17. The protein refractive index increment $\frac{d n}{d c}$ is assumed to be 0.184 for all samples [123]. For full angular readings, where a wide $q$ range is available, data was either fitted using the above procedure, or using Eqn. 5.11 with $z$ fixed at the value obtained from Eqn. 5.17 .

\subsection{Results from Combined SLS and DLS Experiments}

Parts of this section are prepared for a manuscript and have resulted from collaborative efforts with Prof. Klaus Huber and Dr. Carlos Lopez, Paderborn University, Germany.

\subsubsection{Influence of KCL on Kinetics of Vimentin}

Time resolved simultaneous SLS and DLS measurements are performed on the vimentin samples in the concentration range of $0.09 \mathrm{~g} / \mathrm{L}$ to $0.06 \mathrm{~g} / \mathrm{L}$ to track the kinetics of vimentin. Results of the study of $0.07 \mathrm{~g} / \mathrm{L}$ vimentin (initial concentration) upon the influence of $0.05 \mathrm{M} \mathrm{KCl}$ concentration are presented in this section. The initial conditions of the vimentin sample obtained from the measurements at the single-angled SLS setup has the molecular weight $M_{w} \simeq 3 \times 10^{5} \mathrm{~g} / \mathrm{mol}$, radius of gyration $R_{g} \simeq 25 \mathrm{~nm}$ with the mass per unit length $M_{l} \simeq 4700 \mathrm{~g} / \mathrm{mol} /$ nm. The quantities $M_{w}, R_{g}$, hydrodynamic radius $R_{H}$ and the shape sensitive ratio $\rho=\frac{R_{g}}{R_{H}}$ as a function of time are obtained after mixing the KCL into the vimentin sample which are measured at the combined SLS and DLS instrument and presented in Fig. 5.4 .

The values before the addition of salt $(t=0)$ are indicated as large symbols at $t=0.01 \mathrm{~min}$. The first readings correspond to $t \simeq 0.3-0.5 \mathrm{~min}$ after mixing. Significant changes in the state of vimentin are apparent at the earliest time points. The molecular weight is seen to increase to a value of $10^{6} \mathrm{~g} / \mathrm{mol}$, while $R_{g}$ remains constant within experimental error and $R_{H}$ increases by $20 \%$. Further, for $t \leq 10$ $\min , M_{w}$ and $R_{H}$ continue to increase while $R_{g}$ remains constant. This indicates 


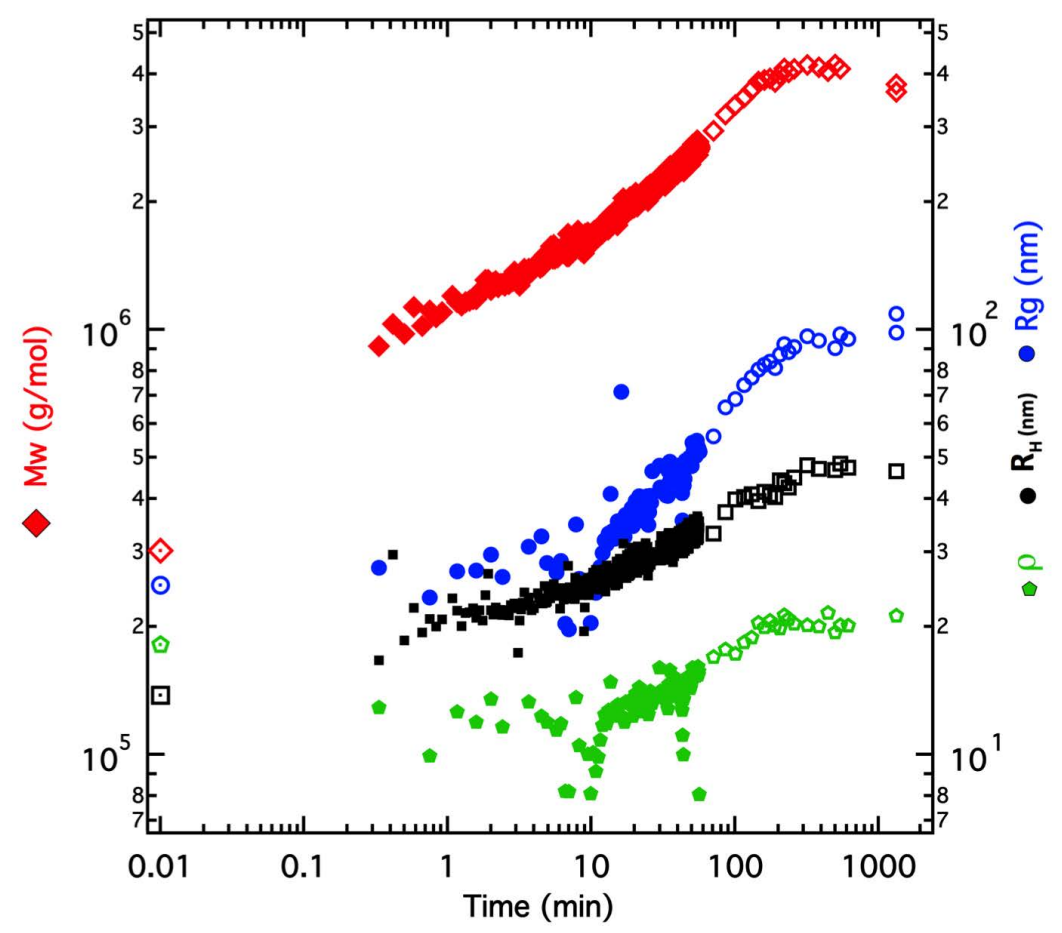

Figure 5.4.: Representative results of the influence of $\mathbf{K C l}$ on vimentin. $M_{w}$ (red diamonds), $R_{g}$ (blue circles), $R_{H}$ (black squares) and $\rho$ (green diamonds) vs. time for a $0.07 \mathrm{~g} / \mathrm{L}$ vimentin solution in $0.05 \mathrm{M} \mathrm{KCl}$. The symbols at $t=0.01 \mathrm{~min}$ correspond to the values of vimentin prior to addition of $\mathrm{KCl}$, i.e. $t=0 . \rho$ is multiplied by a factor of 10 .

that for an assumed rod model, vimentin tetramers undergo lateral assembly for $t \simeq 10 \mathrm{~min}$ and not longitudinal growth. For $t \geq 10 \mathrm{~min}$, the increase of $R_{g}$ and $\rho$ signal elongation and an increase in the aspect ratio of the assembled molecules respectively. At $t \simeq 2 \mathrm{hrs}, M_{w}$ is seen to decrease. Given the length of aggregates at this time is of the order of several hundred nanometers, this is likely to be due to sedimentation. The polydispersity ( $p . d$.) expressed as the ratio of the weight average mass $M_{w}$ and the number average mass $M_{n}$ is found to be constant at a value of 1.3 throughout the experiment. 
Correlation between $M_{w}$ and $R_{g}$

For $t \leq 10 \mathrm{~min}$, the $R_{g}$ data points are spread out and fairly large as shown in Fig. 5.5. However, the values scatter around $29 \mathrm{~nm}$, which is only $15 \%$ higher than the precisely determined value of $25 \mathrm{~nm}$ at $\mathrm{t}=0$, suggesting that growth is dominated by lateral assmebly due to stacking of tetramers for $t \leq 10 \mathrm{~min}$ (plotted as crossed circles). Data for $10 \leq t \leq 60 \mathrm{~min}$ is plotted as filled circles. Data for $t \geq 60$ min, obtained from 30 angle readings with acquisition times of $15 \mathrm{~s}$ per angle and therefore displaying significantly smaller errors are plotted as hollow circles. These longer acquisition times were made possible because the growth process became sufficiently slow at $t=60 \mathrm{~min}$.

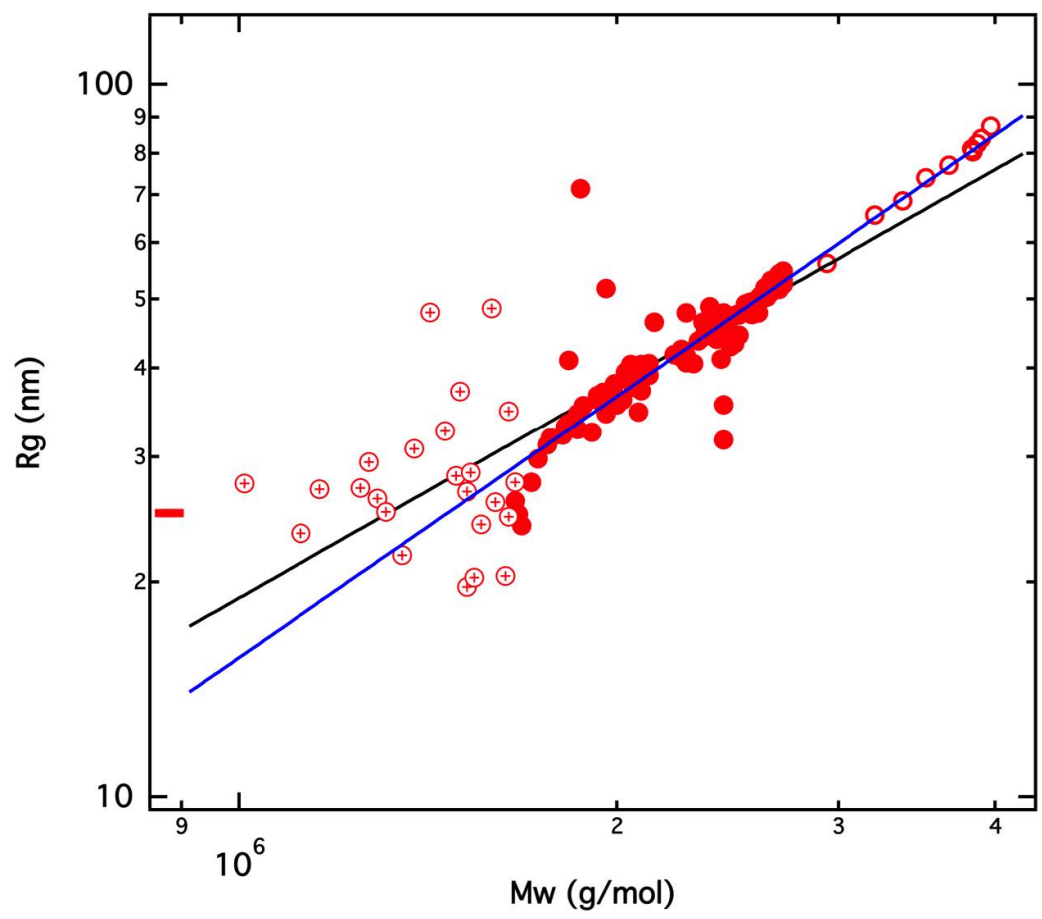

Figure 5.5.: $R_{g}$ vs. $M_{w}$ for the kinetic run from Fig. 5.4. Circles with a cross correspond to $t \leq 10 \mathrm{~min}$ and full circles to $10 \leq t \leq 60 \mathrm{~min}$. Hollow circles correspond to data for $t \geq$ $60 \mathrm{~min}$, which was obtained from full angle readings. The black line corresponds to polydisperse rods with p.d. $=1.3(\mathrm{~m}=3)$ and $M_{L}=21000 \mathrm{~g} / \mathrm{mol}$. The blue line corresponds to a best fit power law. The red bar on the $R_{g}$-axis corresponds to the $R_{g}$ value before the assembly process starts..

A best fit to the rod model with p.d. $=1.3$ and constant mass per unit length yields $M_{L}=21000 \mathrm{~g} / \mathrm{mol} / \mathrm{nm}(\simeq 4.5 \times$ that of the value before addition of $\mathrm{KCl})$. The best fit to a power law yields an exponent of 1.3. Rigid rods may yield 


\section{\begin{tabular}{l|l} 
Chapter $\mathbf{5}$ & TIME-RESOLVED LIGHT SCATTERING FOR VIMENTIN KINETICS
\end{tabular}}

an exponent greater that 1 if either p.d. increases or $M_{L}$ decreases as assembly takes place. If the growth via a monomer addition mechanism is to take place, then the resultant exponent is $\simeq 0.5$ for rods [124] and hence is not the case in our experiment. This mechanistic feature is also confirmed by the correlation between $R_{H}$ vs. $L_{w}$ data shown in Fig. 5.6, $R_{H}$ vs. $L_{w}$ data is well fitted by a cylinder model with $d=30 \mathrm{~nm}$.

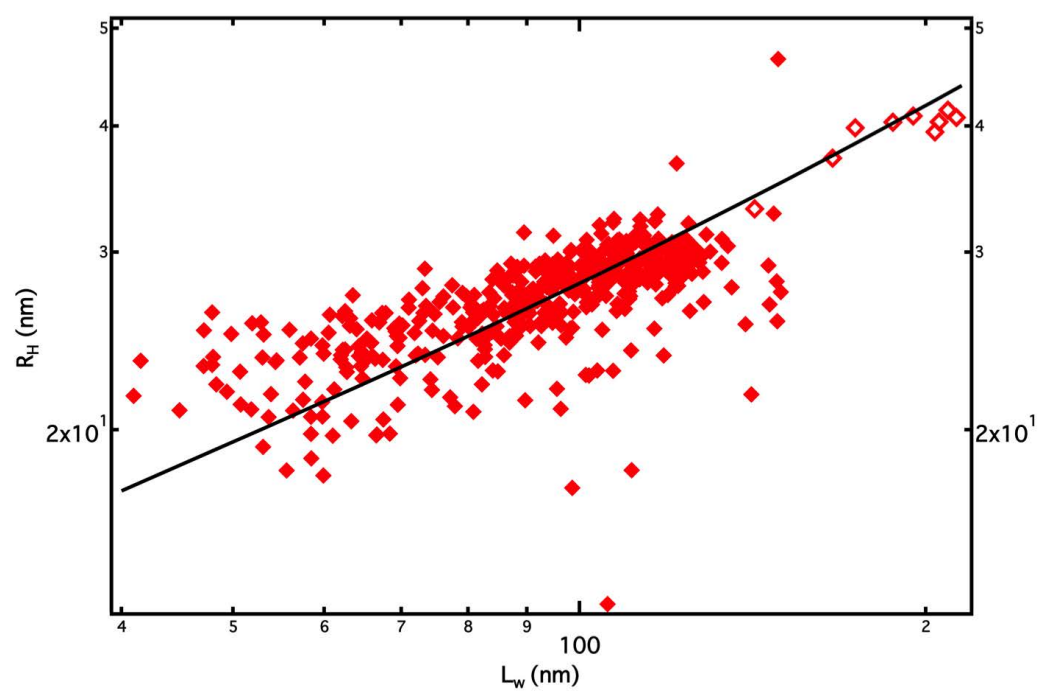

Figure 5.6.: $R_{H}$ vs. $L_{w}$ with model fit. $R_{H}$ vs. $L_{w}$ for the $0.07 \mathrm{~g} / \mathrm{L}$ vimentin kinetic run. The polydispersity is assumed $=1.3$ for all times. Full symbols correspond to measurements with the 8 angle instrument ( $5 \mathrm{~s}$ acquisitions) and the hollow symbols to those taken with the single angle instrument over $\approx 30$ angles (15 s per angle). The black line corresponds to assumption with $d=30 \mathrm{~nm}$.

In conclusion, there is a predominant initial lateral growth of short subunits of vimentin which is followed by a significant elongation of the vimentin filaments due to the influence of $\mathrm{KCl}$. This leads us to a two-step kinetic model for vimentin which is further explored in Sec. 5.3.2.

\subsubsection{Two Step Kinetic Model}

As mentioned earlier, a two step kinetic model is deduced to explain the different stages of vimentin assembly and elongation due to the influence of $\mathrm{KCl}$. Parts of this section are based on the work done in collaboration with Prof. Klaus Huber and Dr. Carlos Lopez, Paderborn University, Germany.

The first stage consists of formation of unit length filaments (ULFs) of vimentin by the lateral assembly process of smaller subunits $p$ at a rate constant $k_{n}$. Here, the 
radius of gyration of the assembling particles does not significantly increase which further confirms lateral assembly. In the second step, these ULFs undergo end-toend elongation with a rate constant of $k_{i, j}$ where the indices $i, j$ depend on the individual ULFs. As well as the filament length, $k_{i, j}$ is a function of $d_{f}$ diameter of the filament and $\delta \omega$, the product of the maximum distance and maximum angle between the ends of two filaments which are required in the elongation step [27, 125, 126, 127, 128]. A schematic diagram of this process is sketched in Fig. 5.8. Following the equation for the diffusion of a cylinder [129, 130]:

$$
D=\frac{k_{B} T}{3 \pi \eta L} \ln (L / d)+0.312+0.565 \frac{d}{L}-0.1 \frac{d^{2}}{L^{2}}
$$

where $d$ is the cylinder's diameter, we can estimate $L_{w}$ and $R_{H}$ and thus obtain the diameter of the smaller sub-units proior to the lateral stacking as $d_{\text {tet }}=10.6$ $\mathrm{nm}$. From these values, the diameter of the ULFs can be estimated as $d_{U L F}=$ $d_{\text {tet }} \sqrt{p}$.

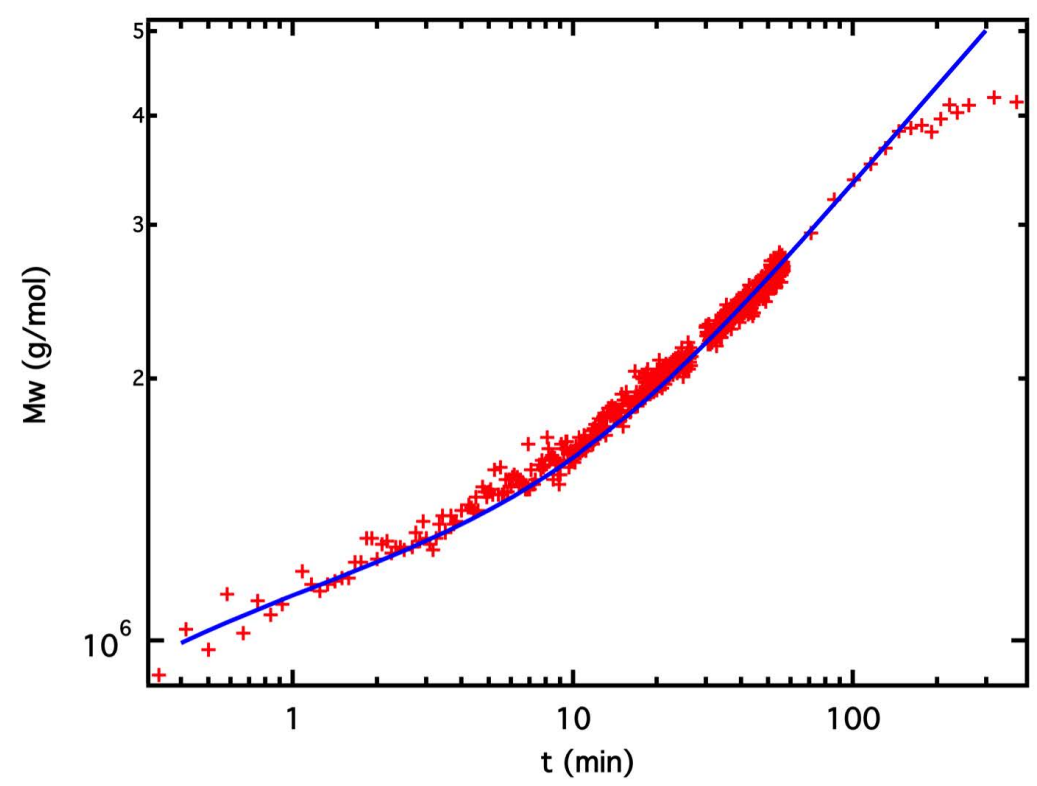

Figure 5.7.: $M_{w}$ vs. time and model fit. $M_{w}$ vs. time for a $0.07 \mathrm{~g} / \mathrm{L}$ vimentin solution in $0.05 \mathrm{M} \mathrm{KCl}$. The blue line shows the model fit for the reaction scheme outlined in Fig. 5.8 The equations used to calculate $M_{w}$ from this model are explained in the Appendix. $D$

In the works of Murphy et al [125, 126], kinetic equations for a mathematical model of aggregation kinetics of Amyloid- $\beta$ has been derived which also explains several stages of fibrillogenesis from smaller subunits to mature fibrils. Following 


\section{\begin{tabular}{l|l} 
Chapter 5 & TIME-RESOLVED LIGHT SCATTERING FOR VIMENTIN KINETICS
\end{tabular}}

this, the solution as shown in Appendix. $\mathrm{D}$ is obtained to fit our kinetic data. In Fig. 5.7, $M_{w}$ is plotted as a function of time (red data points) with a model fit (blue line) which is the solution for the equations considering both the stages of the kinetic process. $k_{n}, \delta \omega$ and $p$ are the three free parameters obtained from the model which is used to describe the experimental data and the values of these are shown in Tab. 5.2. The data display a power law dependency of $M_{w} \propto t^{0.38}$ for long times which is close to the theoretical values of $M_{w} \propto t^{0.33}$ expected for long, thin rods associating end-to-end.

STEP I : lateral stacking

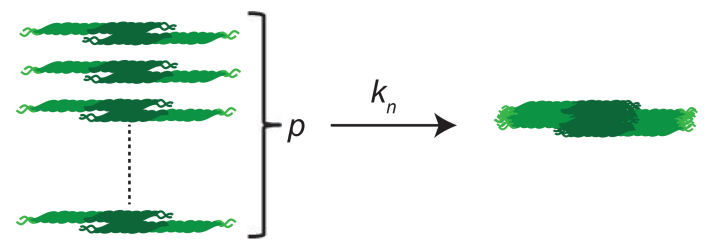

STEP II : end-to-end elongation

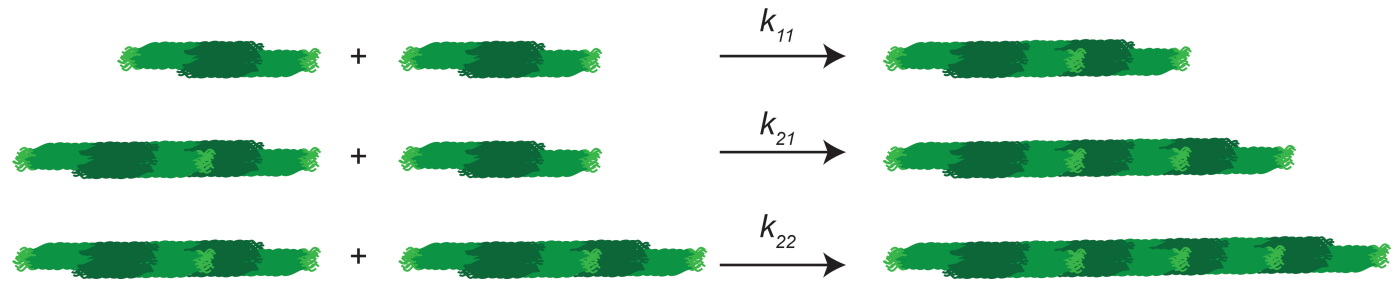

Figure 5.8.: Scheme for the assembly of vimentin.In Step I, $p$ subunits of vimentin laterally stack to form a ULF. In step II, ULFs assembly via an end-to-end association. A ULF may add on to any other species except for the initial subunits. For example, two ULFs can add to form a ULF dimer, a ULF dimer may add another ULF to form a ULF trimer, two ULF-dimers can assemble to form a ULF tetrameter etc. Adapted with permission from [108]

\subsubsection{Results for Variabiltiy in Vimentin Concentration}

Other concentrations of vimentin $\left(c_{p}=0.09\right.$ and $\left.0.06 \mathrm{~g} / \mathrm{L}\right)$ are studied at $c_{s}=0.05 \mathrm{M}$ $\mathrm{KCl} . M_{w}$ vs. time for the three different concentrations are plotted at shown in Fig. 5.9a. A significant variation in the kinetics between the 0.07 and the $0.09 \mathrm{~g} / \mathrm{L}$ samples can be observed, which is probably caused not only by the difference in concentration and starting parameters. $R_{g}$ vs. $M_{w}$ for the three kinetic runs is shown in Fig. 5.9 , along with best fit lines to $R_{g}=k M_{w}{ }^{1}$, which is the functional form expected for rigid rods of constant polydispersity. The mass per unit length 
may be estimated from the value of $k$ given a value for the polydispersity, which we assume to be the same as the initial value for the smaller subunits (i.e. before addition of salt) . The $M_{L}$ values for are within $20 \%$ of the value obtained from fitting the kinetic model for the 0.06 and $0.07 \mathrm{~g} / \mathrm{L}$ samples. The kinetic model fit to the $0.09 \mathrm{~g} / \mathrm{L}$ sample yields an $M_{L}$ value $50 \%$ higher than the calculated one in Fig. $5.9 \mathrm{p}$. The kinetic process remains qualitatively the same for all samples as seen in Fig. 5.9k-d: first, a lateral assembly step occurs, as signaled by a large increase in $M_{w}$ and $R_{H}$ without any significant increase of $R_{g}$. Eventually, the increase in $R_{g}$ coupled with an increase in the $\rho$ parameter signals elongation, with a $M_{w} \propto t^{0.33}$ relation that is characteristic for end-to-end association of rods.
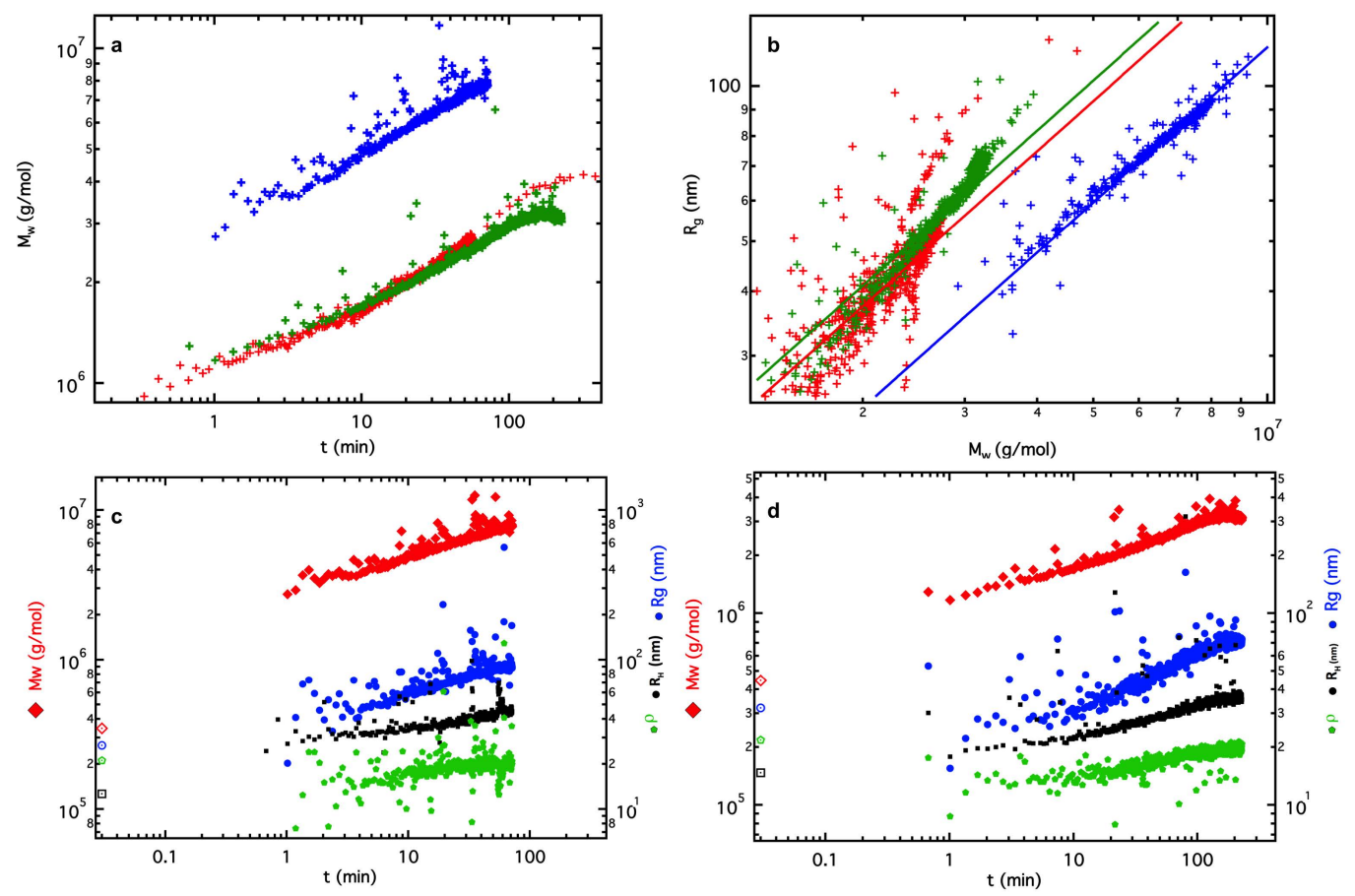

Figure 5.9.: $M_{w}$ vs. time for 3 different kinetic runs.a) Red symbols $c_{p}=0.09 \mathrm{~g} / \mathrm{L}$, blue symbols $c_{p}=0.07 \mathrm{~g} / \mathrm{L}$, green symbols $c_{p}=0.06 \mathrm{~g} / \mathrm{L}$. b) $R_{g}$ vs. $M_{w}$, symbols have the same meaning as in a). c) $M_{w}$ (red diamonds), $R_{g}$ (blue circles), $R_{H}$ (black squares) and $\rho$ (green stars) for the kinetic run with vimentin concentration of $0.09 \mathrm{~g} / \mathrm{L}$. Large symbols at $t=0.03 \mathrm{~min}$ represent values before addition of $\mathrm{KCl}$. d) Same as c) but for $0.06 \mathrm{~g} / \mathrm{L}$ vimentin concentration.

The values of $p, k_{n}$ and $\delta \omega$ increase with increasing vimentin concentration as shown in Tab. 5.2. The resulting mass per unit length of the ULFs, which varies from 22 to $50 \mathrm{~kg} / \mathrm{mol} / \mathrm{nm}$ corresponds to $\simeq 6-16$ tetramers per ULF. The fit to the kinetic model suggests $10 \%$ of the tetramers are unreacted after $2 \mathrm{hrs}$ for the $0.06 \mathrm{~g} / \mathrm{L}$. The initial assembly step is too fast for the $0.09 \mathrm{~g} / \mathrm{L}$ sample to 


\section{\begin{tabular}{l|l} 
Chapter 5 & TIME-RESOLVED LIGHT SCATTERING FOR VIMENTIN KINETICS
\end{tabular}}

accurately model the initiation step and we therefore cannot estimate the level of unconsumed subunits. A sample with $c_{P}=0.015 \mathrm{~g} / \mathrm{L}$ and $c_{S}=0.05 \mathrm{M}$ did not assembly. This suggests that a minimum protein concentration is required for assembly to take place, which is not taken into account in our simple kinetic model. This finding is consistent with our fit to the $0.07 \mathrm{~g} / \mathrm{L}$ and to the 0.06 $\mathrm{g} / \mathrm{L}$ samples as these fits account that about $10 \%$ of the subunits do not undergo assembly in $\simeq 2 \mathrm{hrs}$.

Table 5.2.: $M_{w}, R_{g}, R_{H}$ and $M_{L}$ for samples used for kinetic runs $M_{w}, R_{g}, R_{H}$ and $M_{L}$ for samples used for kinetic runs and parameters from fits to the kinetic model $c_{S}=0.05 \mathrm{M}$ and varying $c$.

\begin{tabular}{crrrrrrr}
\multicolumn{3}{c}{ Samples in sodium phosphate buffer } & \multicolumn{3}{c}{ Kinetic parameters } \\
\hline$c(\mathrm{~g} / \mathrm{L})$ & $M_{w}(\mathrm{~g} / \mathrm{mol})$ & $R_{g}(\mathrm{~nm})$ & $R_{H}(\mathrm{~nm})$ & $p$ & $\delta \omega(\mathrm{mrad} \mathrm{nm})$ & \multicolumn{1}{c}{$k_{n}\left(\mathrm{M}^{p-1} / \mathrm{s}\right)$} & $M_{L}\left(\times 10^{3} \mathrm{~g} /(\mathrm{mol} \mathrm{nm})\right)$. \\
\hline $0.09 \pm 0.01$ & $3.5 \times 10^{5}$ & 27 & 13 & $9 \pm 1$ & $400000 \pm 100000$ & $\sim 10^{57}$ & 45 \\
$0.07 \pm 0.02$ & $3 \times 10^{5}$ & 25 & 14 & $6 \pm 1$ & $80000 \pm 20000$ & $5 \times 10^{33}$ & 29 \\
$0.06 \pm 0.01$ & $4.2 \times 10^{5}$ & 32 & 15 & $5 \pm 1$ & $50000 \pm 10000$ & $2 \times 10^{28}$ & 25 \\
\hline
\end{tabular}

\subsection{Summary and Closing Remarks}

The kinetic process of tetrameric vimentin upon the influence of a monovalent salt is characterized using a combined time resolved SLS and DLS technique. As a representative result, the kinetics of a $0.07 \mathrm{~g} / \mathrm{L}$ vimentin sample upon mixing with salt concentration $0.05 \mathrm{M} \mathrm{KCl}$ aqueous solution are found to proceed in two stages: lateral assembly dominates growth for the first 10 minutes and end-to-end association dominates for larger times. Data for $M_{w}, R_{g}$ and $R_{H}$ can be adequately described by a two step model following the work of Murphy et al [126]. The model gives a satisfactory fit to the data, however, several important approximations must be noted: 1) we have assumed all reactions to be irreversible, 2) lateral assembly (step I) is assumed to occur for only a single value of $p$, while in fact it is well known that vimentin filaments exhibit heterogeneous $M_{L}$ values [34, 131]. 3) the mean value of $L$ is used to calculate the reaction constant for step II, this avoids having to solve a large number of differential equations. 4) we employ an approximate expression for the diffusion coefficients of rods, which may suffer at low aspect ratios 5) we do not take into account the fact that a 'threshold concentration' for assembly may exist. However, small variations in concentration lead to large variations in the fit parameters, although the assembly process remains qualitatively the same. 


\section{Summary and Conclusion}

Understanding the assembly kinetics of vimentin intermediate filaments on various length scales from nanometers to micrometers is the central aim of this thesis. In order to obtain detailed information, we employ analytical and non-destructive imaging techniques such as small angle X-ray scattering (SAXS) and light scattering (LS) to get results over a accessible resolution range in the reciprocal space [55, 80, 115]. The results from the two imaging techniques is dealt in detail in the previous chapters where assembly and aggregation of vimentin in the presence of mono- and divalent salts is methodically combined with (i) non-segmented continuous flow microfluidics, (ii) segmented droplet microfluidics, (iii) finite element methods (FEM) simulations to complement flow conditions, (iv) combined application of time-resolved several-angled LS detection and finally (v) mathematical formulation of a kinetic model. In this thesis, we strive to answer the necessity of studying the assembly process of vimentin from a biophysical context, where vimentin plays a crucial role in the mechanics of the eukaryotic cells of mesenchymal origin. What is intriguing is its hierarchical structural architecture. Since the emerging assemblies that make up the full-length filament are on the order of nanometers in diameter, an in situ approach is applied by a combination of microfluidics, X-ray and light scattering techniques to answer our scientific question. 


\section{\begin{tabular}{l|l} 
Chapter 6 & SUMMARY AND CONCLUSION
\end{tabular}}

In the first part of the thesis, we develop different microfluidic devices which are beneficial in the SAXS studies, where:

- for continuous flow experiments, a serial-inlet device geometry is designed in order to mix in the $\mathrm{KCl}$ and $\mathrm{MgCl}_{2}$ salts subsequently to functionally trigger the assembly of the non-filamentous vimentin and observe the effect in a time resolved manner.

- for droplet experiments, $\mathrm{KCl}$ solution and tetrameric vimentin together with the assembly buffer are pinched off into aqueous-in-oil emulsions in an Xray/droplet compatible device to study the kinetics of vimentin assembly at stable concentrations.

In the second part of the thesis, the focus is on the combination of SAXS and the state-of-the-art microfluidic devices for tracking kinetics.

- The combination of SAXS and continuous flow microfluidics enables us to investigate the assembling structures of vimentin on the nanometer scale. Simultaneously, the FEM simulations performed, shed light on the flow conditions and track the change in concentration of the in-flow species of the device. By analyzing the streamlines of the flow in the laminar regime from the simulations, we arrive at the relevant mixing and reaction kinetics occurring at the temporal resolution on the order of seconds. With the use of SAXS, we have probed the surface of the nanometer vimentin assemblies with a micrometer sized beam in the continuous flow experiment. Appearance of isotropic scattering signal around the primary beam along the flow in the channel hints at a very non-orientational development of the laterally associated vimentin filaments. However, with the given resolution as explained in Sec. 4.4.1, information of the compactness of the surface of these developing filaments due to the influence of $\mathrm{K}^{+}$and $\mathrm{Mg}^{2+}$ ions can be understood.

- The droplet-mapping with SAXS, however, is a demonstrative technique for a fully controlled time-series detection method of vimentin assembly at constant concentration. The combination of droplets and SAXS with the various measurement techniques is demonstrated as a proof-of-principle to study weakly scattering proteins, of which the fast acquisition with shorter exposure times is the most preferred method. The fast acquisition technique gives a signal-to-noise ratio which is possible with the exclusion of signal from the anisotropic interfaces and fluorocarbon oil during data reduction. 
The third part of the thesis focuses on obtaining the 'missing' information of vimentin kinetics with light scattering. We obtain information of a "specific" stage of the vimentin elongation during assembly with a combined SLS and DLS detection method. By characterizing the assembly of vimentin in the presence of a monovalent salt, we observe a two step kinetic process including the lateral assembly that occurs between the times of 10 to $60 \mathrm{~min}$ and subsequently the end-to-end assembly that occurs at larger time scales until no further reaction is seen in the mature filaments.

\section{Advantages and Challenges of Our Selected Methodology}

Several advantages can be observed in the techniques we adopt to study protein kinetics, which can be generalized to other systems as well. They are:

- The combination of X-ray and light scattering can probe different length scales of the ongoing kinetics, thus encompassing a good spatial resolution of the protein system on the order of nanometers upto $600 \mathrm{~nm}$.

- Single and multi-phase microfluidics is a good lab-on-chip environment for the X-ray detection otherwise which the biological system under study might undergo radiation damage. Additionally, another advantage is that by probing at different positions on the length of the channel during flow, local temporal resolution can be obtained.

- The custom fabricated droplet-microfluidic device can find its application in free electron laser (FEL) experiments where a liquid-jet containing the protein system can be injected and imaged before destruction. Since the device can withstand very high flow rates, it is suitable for free-jets which are injected to match the frequency of the detector read-out in FEL experiments. Additionally, other biological particles such as independently flowing red blood cells or even crystallized proteins can be imaged over several days without any damage [69].

- Tracking the ion-induced assembly kinetics of vimentin with a combination of these techniques serves as a model to study similar/other biopolymer systems.

However, in the scope of this thesis, several challenges have been encountered. Some of the main issues with plausible solutions are discussed here: 
- The NOA-81 devices fabricated for the continuous-flow SAXS experiments are prone to radiation damage and leakage, eventually leading to clog and disruption of the experiment. As a solution to this, devices compatible with the droplets (made of composite PDMS-quartz capillary) were fabricated to circumvent these problems.

- However, initial kinetics cannot be tracked with the droplet device, since a dead time of a few milliseconds 500-900 ms, values calculated based on the channel dimensions exists between the pinch-off and the first point of droplet-detection. As a possible solution to this, a new device with cyclic olefin copolymers (COC) commercially available as TOPAS can be fabricated to access the mixing of protein and the salt and track the initial-reaction assembly steps.

- X-ray data obtained in the transmission mode of SAXS reveal information of the size, shape and surface of the biomolecules. Inappropriate determination of the resolution or $q$-range during experiments leads to discrepancies in data analysis. Therefore, as a possible solution, stationary experiments in capillaries are suggested to get a preliminary idea of the size-parameters of the particles.

- The calculated volume of the protein solutions in microfluidic channels are on the order of nanolitres. Additionally, the beam sizes are on the order of $\approx 5 \mu \mathrm{m} \times 5 \mu \mathrm{m}$. This setup often does not give information of higher structural organization at the extended $q$-ranges. As a possible solution to circumvent these issues, a channel for higher volume containment, a larger beamsize or even the application of macrofluidic setups are suggested. These improvements are also beneficial in the droplet experiments where the entire droplet is imaged which is more-or-less the same beamsize to get a better ensemble average.

- Light scattering measurements give a size-parameter by several approximation theories i.e., Guinier approximation, Zimm approximation etc. However, large sample volumes are required for filtering and measurement. Also, the samples used have a low protein concentration which are ideal for the detectors but not for tracking the kinetics of the protein. As a solution, optimization of the threshold of detectors have to be made so that higher concentrations of protein can be used. This may yield better results and 
statistics with respect to protein kinetics.

\subsection{Open Questions and Outlook}

With respect to the experimental procedures, a few open questions can be addressed:

- Can longer time scales be accessed with SAXS and microfluidics? A combined information for the compaction and elongation of vimentin is missing in th real and reciprocal space. What happens at the surface during the elongation process? What are the molecular changes in the compaction stage? Does it just follow the hypothesized binding to the head groups and freetails or is the polyelectrolyte nature the only effect seen? At physiological conditions, while the surface changes, does it follow a self-similarity condition in accordance with the fractal dimensions? Then, another unknown parameter would exist. In order to satisfy the self-similarity condition, the same exponents can be found for a magnitude higher. It is therefore necessary to get more information on higher orders of the $q$-range. This can be done with a combined WAXS/SAXS setup for which several beamlines are equipped. Effect of divalent ions plays a major role in the onset of aggregation kinetics into network like structures, which can further be studied with SAXS at different resolutions.

- Critical concentration of protein versus critical concentration of salt is really a crucial observation. Does low protein concentration mediate assembly or not at a critical concentration of the salt?

- A similar assortment of techniques can be applied to study the kinetics of other modified vimentin types, like the $Y 117 \mathrm{~L}$ vimentin mutant. Perhaps, this helps us to understand better the polyelectrolyte nature of the assembly process as to what really happens in the head and the tail regions.

- The kinetic model proposed from the light scattering data accounts for heterogeneity, which can be seen with either TEM or other high resolutions imaging techniques such as SAXS but not for flexibility. Perhaps, we can think about adapting a flexibility parameter by introducing a polydisperse worm-like chain model according to the theoretical considerations in [132], 
unlike the rigid cylinder in the present model. Additionally, another question can be contemplated: Can disassembly be tracked with X-rays? Or with light scattering? Because the kinetic model proposed from the light scattering data lets us assume that the assembly process is irreversible. If however, disassembly could be tracked, the data could be modeled with derivation of additional rate constants as mentioned in [127] or as fractal aggregates [133].

\subsection{Structural Quantification of Vimentin}

a)

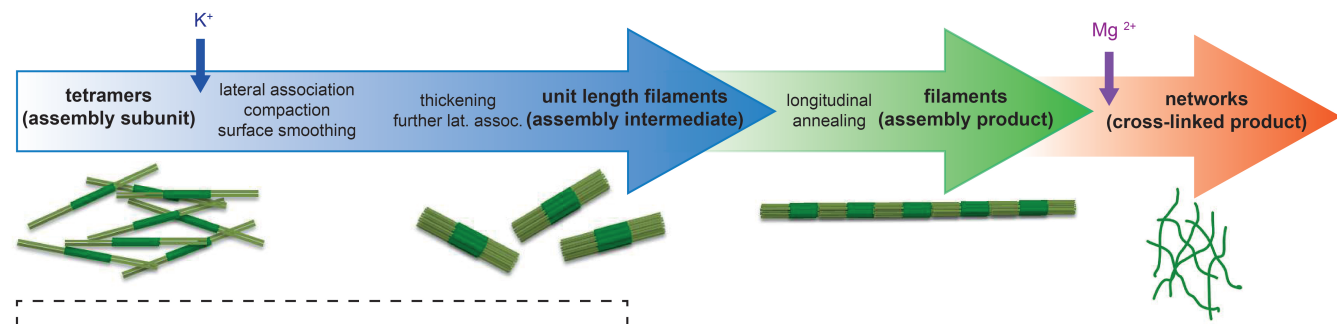

b)

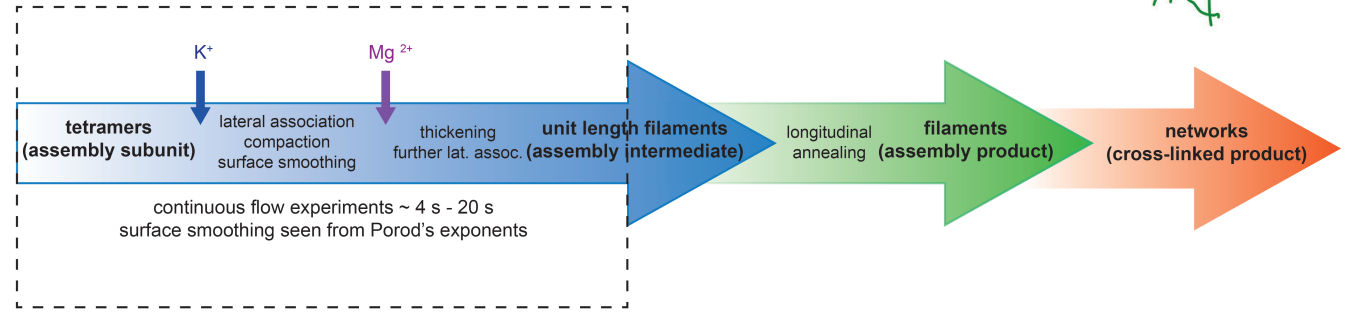

c)

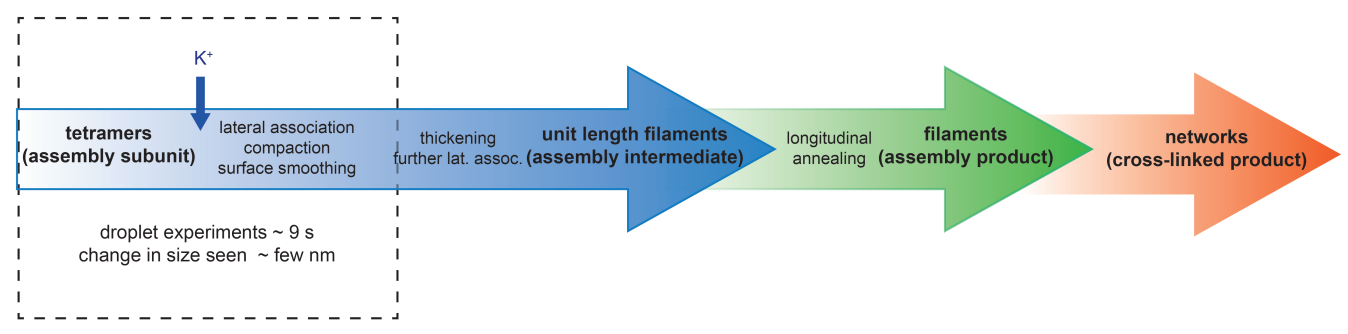

d)

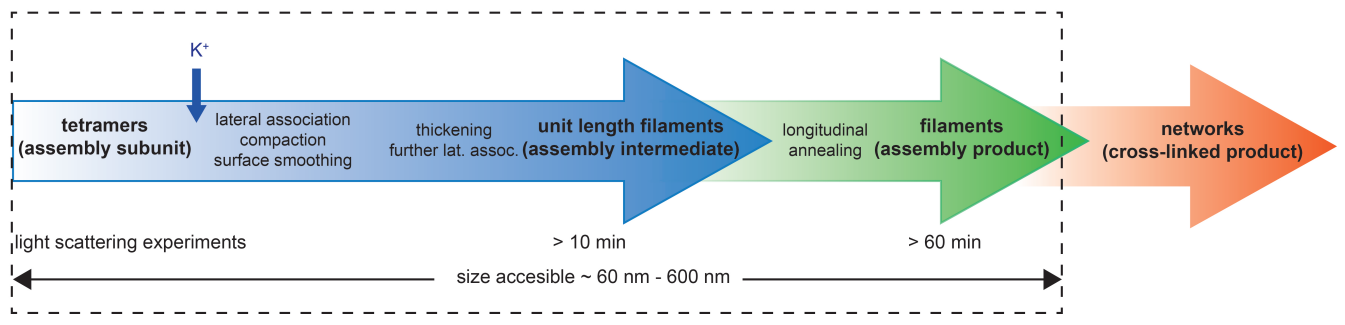

Figure 6.1.: Overview scheme of the length and time scales accessible with our methods. a) Hierarchical assembly and cross-linking mechanism of vimentin IFs upon the addition of monoand divalent salts. b) Information obtained from continuous flow experiments. c) Information from droplet experiments. d) Information obtained from light scattering experiments. 
Our findings of the accessible length and time scales of vimentin kinetics are summarized in the Fig. 6.1. Hierarchically, vimentin tetramers form filaments through an intermediate stage of ULFs in the presence of a monovalent salt and further cross-link to form networks in the presence of a divalent salt as shown in Fig. 6.1 1 . The maximum time accessible with our continuous-flow experiments (see Fig. 6.1p) is upto $20 \mathrm{~s}$, based on different flow rates. We obtain information not of the size but of surface of the emerging vimentin assemblies. The surface of the particles change from an rough exterior and transitions into a smoothening phase. A critical concentration of $\mathrm{KCl}$ is necessary to unblock the formerly unproductive head domains and, thus, initiate assembly [63]. $\mathrm{MgCl}_{2}$ plays minimal role in the surface properties but further engages in the thickening and lateral assembly of vimentin. Our analysis of the surface sensitive Porod regime in the Xray data reveals that the formation of first assembly intermediates, so-called unit length filaments, is not a one-step reaction but consists of distinct consecutive lateral association steps followed by radial compaction as well as smoothening of the surface of the full-width filament [63]. Perhaps, with a longer channel length, the state of the ULFs without the influence of a divalent salt can be better investigated. With the droplet-based experiments of tracking vimentin assembly, we can access the time points upto $\approx 9 \mathrm{~s}$ as shown in Fig. 6.1k. The size of the emerging vimentin assemblies is of a few nanometers in diameter which is similar to the previously reported experiments [35]. The focus of the experiment is to establish a state-ofthe-art high resolution technique and vimentin assembly is tracked as a model system.

With the LS experiments, bulk samples are investigated where tetramers stack to form ULFs until $10 \mathrm{~min}$ (see Fig. 6.1 d). The elongation step, then occurs for longer time-scales. This contrasts with other reports [34, 35], which suggest that lateral assembly is complete after a few seconds. The results of Hermann et al.[34], Krimse et al. [31] and Portet et al. [27] were taken at $37{ }^{\circ} \mathrm{C}$ and at higher salt concentrations $(0.1-0.16 \mathrm{M})$ and protein concentrations and are, thus, not directly comparable to our data. Additionally, our data are collected using a non-invasive technique, unlike the previous references which further complicates comparison. In comparison, when we assembled vimentin with the "kick-start" mode by mixing the protein and salt in an eppendorf tube, we incubate it for 15 hours, just so the end result is fully formed mature filaments although the elongation has long stopped. Once the process saturates, the elongation no longer occurs which we see from the sedimentation of the filaments in the solution from light scattering. 
Therefore, the goal of tracking assembly kinetics of vimentin is achieved by a combination of microfluidics, X-rays and light scattering in a novel way to track different length and time scales. The advantage of combining SAXS and LS is that the initial assembling steps until the formation of ULFs can be seen with the Xrays and the elongation step can be seen in light scattering, since X-ray "cannot" visualize particles above its resolution limit. Protein molecules are dynamic in vivo and this encourages us to study the vimentin assembly in a "dynamic" condition, which is achieved in flow with the microfluidic device in the present study. The combination of these techniques can be further extended to study similar systems such as other IF proteins, amyloid proteins (since the diameter of the fibrils are on the same range as IFs) etc. 


\section{Purification of Vimentin}

This section gives a detailed protocol for the expression and purification of vimentin in Escherichia coli bacteria.

\section{Cultivation of E. coli}

- One vial $100 \mu \mathrm{L}$ E.coli (T1 cells, \#T3017, Zymo research, Irvine, California, USA; stored at $-80^{\circ} \mathrm{C}$ ) is thawed on ice for 3 min and directly transfected by the addition of $1 \mu \mathrm{L}$ plasmid with gentle mixing.

- $100 \mu \mathrm{L}$ of the bacteria is then incubated for $5 \mathrm{~min}$ on ice and $50 \mu \mathrm{L}$ of the bacteria solution is spread out on a warm lysogeny broth (LB) agar plate (SIGMA L5667). The agar plate is warmed in an incubator (Ecotron, Infors $\mathrm{HT}$, Bottmingen, Germany) at $37^{\circ} \mathrm{C}$ over night.

- Three bacteria colonies of $1 \mathrm{~L}$ each are chosen in separate autoclaved terrific broth solution (47,6 g TB (SIGMA, \#T0918) and $8 \mathrm{~mL} \mathrm{99,5 \%} \mathrm{glycerol).} 50 \mathrm{ml}$ of one of those colonies is chosen and mixed with the start culture with $5 \mu \mathrm{L}$ ampicillin solution $(100 \mathrm{mg} / \mathrm{mL}$ ampicilin sodium salt, final concentration 1:1000, SIGMA) and grown for $6 \mathrm{~h}$ at $37^{\circ} \mathrm{C} / 150 \mathrm{rpm}$ in the incubator. After- 
wards, each bacteria solution is transferred to 11 terrific broth solution (with $0.5 \mathrm{~mL}$ ampicillin solution) and let it to grow at $37^{\circ} \mathrm{C} / 150 \mathrm{rpm}$ over night.

- The bacteria are then separated from the growth medium by 15 min centrifugation at $4500 \mathrm{~g} / 4^{\circ} \mathrm{C}$ (Eppendorf centrifuge $5810 \mathrm{R}$, Eppendorf, Hamburg, Germany). The bacteria pellet is stored at $-20^{\circ} \mathrm{C}$ until purification.

\section{Purification Step}

- The bacteria pellets are dissolved in a total of $48 \mathrm{~mL}$ lysis buffer and separated into two 'dounce homogenizers' (20.05 $\mathrm{mm} \pm 0.025 \mathrm{~mm}$ pestle, VWR, Darmstadt, Germany). $6 \mathrm{ml}$ lysozyme solution is added to each homogenizer and the pellet is homogenized and incubated for $30 \mathrm{~min}$ on ice.

- Afterwards, $300 \mu \mathrm{L} \mathrm{1M} \mathrm{MgCl} 2,33 \mu \mathrm{L}$ RNaseA solution $(1 \mathrm{mg} / \mathrm{mL}), 30 \mu \mathrm{L}$ DNase1 solution (50 mg/mL), $300 \mu \mathrm{L}$ PMSF (Phenylmethylsulfonylfluorid solution in ethanol) saturated solution and $600 \mu \mathrm{L} 10 \mathrm{v}-\%$ NP40 solution are added to each homogenizer, the solution is homogenized again and incubated on ice for additional $10 \mathrm{~min}$. Then $60 \mathrm{ml}$ detergent buffer, $600 \mu \mathrm{L} 1$ M DTT, $900 \mu \mathrm{L}$ PMSF solution and $300 \mu \mathrm{L} 50 \mathrm{mM}$ Pefabloc are added to each homogenizer, and following another homogenization step the solution is centrifuged in 4 centrifuge tubes for $25 \mathrm{~min}$ at $10000 \mathrm{~g}$ and $4{ }^{\circ} \mathrm{C}$ (Beckmann centrifuge J26XP, Rotor Ja $30.50 \mathrm{Ti}$, Beckmann Coulter GmbH, Krefeld, Germany).

- This is followed by washing and centrifugation step 1 , where the supernatant is decanted, the pellets are dissolved in a total of $120 \mathrm{~mL}$ GII buffer, $120 \mu \mathrm{L}$ $1 \mathrm{M}$ DTT, $600 \mu \mathrm{L}$ PMSF solution and $300 \mu \mathrm{L} 50 \mathrm{mM}$ and $1.5 \mathrm{M} \mathrm{KCl}$ Pefabloc homogenized and incubated on ice for $30 \mathrm{~min}$.

- The solution then is separated into four centrifuge tubes and centrifuged for $20 \mathrm{~min}$ at $10000 \mathrm{~g}$ and $4{ }^{\circ} \mathrm{C}$ (Beckmann centrifuge J26XP). In washing and centrifugation step 2, again, the supernatant is decanted, the pellets are soluted in a total of $120 \mathrm{~mL}$ GII/KCl buffer, $120 \mu \mathrm{L} 1 \mathrm{M}$ DTT, $600 \mu \mathrm{L}$ PMSF solution and $300 \mu \mathrm{L} 50 \mathrm{mM}$ Pefabloc homogenized and incubated on ice for $10 \mathrm{~min}$.

- The solution then is separated into four centrifuge tubes and centrifuged for 
another $20 \mathrm{~min}$ at $10000 \mathrm{~g}$ and $4{ }^{\circ} \mathrm{C}$. The subsequent washing and centrifugation step 3 is identical to washing and centrifugation step 1 .

- In washing and centrifugation step 4, the pellets are dissolved in $80 \mathrm{~mL}$ TE buffer, $80 \mu \mathrm{L} 1 \mathrm{M}$ DTT, $400 \mu \mathrm{L}$ PMSF solution and $200 \mu \mathrm{L} 50 \mathrm{mM}$ Pefabloc, homogenized, incubated on ice for $15 \mathrm{~min}$ and centrifuged in two centrifuge tubes for $10 \mathrm{~min}$ at $10000 \mathrm{~g}$ and $4{ }^{\circ} \mathrm{C}$.

- The pellet is dissolved in $28.5 \mathrm{~mL} 9.5 \mathrm{M}$ Urea, $300 \mu \mathrm{L} 1 \mathrm{M}$ TRIS-HCl (pH 7.5), $300 \mu \mathrm{L} 0.5$ M EDTA, $300 \mu \mathrm{L}$ PMSF solution and $300 \mu \mathrm{L} 1 \mathrm{M}$ DTT and homogenized. The solution then is centrifuged for $1 \mathrm{~h}$ at $31170 \mathrm{rpm}(\approx 100000 \mathrm{~g})$ at $20{ }^{\circ} \mathrm{C}$ (Beckmann-UZ L90K, Ti70 rotor, Beckmann Coulter GmbH, Krefeld, Germany).

- The supernatant is kept, $10 \mu \mathrm{L} 1 \mathrm{M}$ MAC solution are added per millilitre supernatant and it is stored over night at $-20^{\circ} \mathrm{C}$ and then gently thawed before the ion exchange chromatography.

\section{Ion Exchange Chromatography}

\section{Part 1 - anion exchanger}

The ion gradient mixer ( $2 \times 50 \mathrm{ml}$, Schütt 24, Göttingen, Germany) is filled with $40 \mathrm{~mL}$ column buffer in the front chamber and $40 \mathrm{~mL}$ column buffer with 0.448 $\mathrm{g} \mathrm{KCl}$ added. The DEAE sepharose is filled into the chromatography column (Econo chromatography column, $25 \mathrm{~mm}$ diameter, $200 \mathrm{~mm}$ long, Biorad, Munich, Germany), allowed to settle and carefully washed with $40 \mathrm{~mL}$ column buffer. Then the protein solution is added and the column is adjusted such that there is a slow ow with a constant filling level of the column. The out ow from the column is collected. The column is once again washed with $40 \mathrm{~mL}$ column buffer (slowly and at a constant level). When the collected total out ow volume of the column reaches the dead volume of the column the actual sample collection begins. After the washing, the gradient mixing is started and the gradient solution flows into the column at about 3 to 4 drops per second. The flow into and out of the column are then adjusted such that the volume in the column remains constant and the outflow is in the range of 7 to 9 drops per second. The out ow is collected in partitions of about $1.5 \mathrm{~mL}$ and each partition is monitored for its protein content 


\section{\begin{tabular}{l|l} 
Appendix & PURIFICATION OF VIMENTIN
\end{tabular}}

(see sec. 2.3). The partitions containing protein are kept and combined for the cation exchanger column.

Part 2 - cation exchanger

The cation exchange column chromatography is analogous to the anion exchange column chromatography except that the column material is CM sepharose instead of DEAE sepharose and that the salt concentration in the gradient mixer is twice as high. After the cation exchanger $10 \mu \mathrm{L} 1 \mathrm{M}$ MAC solution are added per millilitre sample and the sample is stored at $-80^{\circ} \mathrm{C}$.

\section{Quality Control}

The purity of the extracted vimentin is controlled by an SDS (sodium dodecyl sulfat) page gel.

\section{Buffers}

- electrophoresis buffer: 0.4 M Tricine, 0.6 M TRIS, 1 \% SDS, 25 mM Natriumhydrogensulfit at $\mathrm{pH}$ 8.2-8.3

- sample buffer: $150 \mu \mathrm{L} 4$ x sample buffer for gels (VWR/CBS, ordering no. FB31010), $270 \mu \mathrm{L} \mathrm{H2O}, 60 \mu \mathrm{L}$ DTT $10 \times$ (VWR/CBS, ordering no. 32001)

\section{Gel run}

A CBS gel (TEO) with $8 \%$ acrylamide (VWR/CBS, ordering no. FK00812-10) is mounted in DCX 700 gel chamber (VWR). $10 \mu \mathrm{L}$ each of sample and controls of interest are mixed with $40 \mu \mathrm{L}$ sample buffer and denatured for $10 \mathrm{~min}$ at $70{ }^{\circ} \mathrm{C}$. Afterwards the solutions are quickly centrifuged (Eppendorf MiniSpin, Eppendorf, Hamburg, Germany) and $10 \mu \mathrm{L}$ from the top are placed in one lane of the gel. On at least one of the lanes, a gel marker ("Protein Marker", pre-stained 6.5-200, AppliChem, Darmstadt, Germany) is run. The electrophoresis is performed at a maximum voltage of $200 \mathrm{~V}$ and a constant current of about $100 \mathrm{~mA}$ until the bands are well seperated (roughly $1.5 \mathrm{~h}$ ). The finished gel is dyed with InstantBlueTM (Expedeon, San Diego, California, USA) for 15 to 60 minutes and subsequently rinsed with water to remove excess dye. A single, isolated band at $53 \mathrm{kDa}$ indicates purified vimentin. 


\section{Matlab Code for Evaluating Time Evolution from COMSOL 5.0}

In Chapter. 3, we track the kinetics of vimentin by converting the spatial coordinates of a microfluidic channel into time co-ordinates. This is performed by a LiveLink for MATLAB provided by COMSOL, where the FEM simulations are imported and the data is post-processed using a code. The algorithm for the code exists in 5 different steps where at the end the time-evolution along a streamline is calculated and the output is in a readable data format. The output data time vs. length presented in this thesis is plotted in OriginPro. 8.5G. Parts of the MATLAB code are provided in this section for easy understanding of the algorithm. 


\section{Part1: Marking Streamline Co-ordinates}

The first step is to identify the required streamline, in our case the streamline at the center or at close proximity to the central focused jet. This can be either calculated in COMSOL or in MATLAB after importing. The cut-lines in the geometry of COMSOL help in easy identification of the streamline co-ordinates.

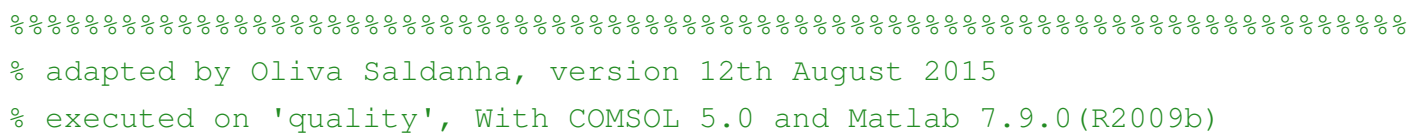




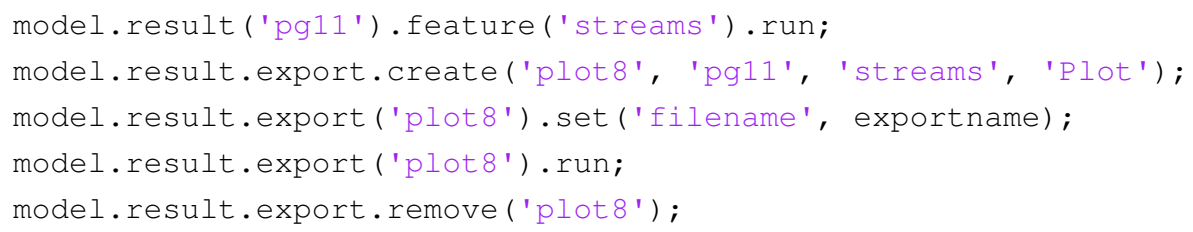

\section{Part2: Evaluation Along the Streamline}

Once information of the streamline co-ordinates are obtained, the flow field and the local concentrations at each position can be obtained in this second step. This step has to be compiled before any further calculations are performed. Without the information from the concentration at each position, the integration alone the streamline might only give time, but only for the ideal case. For the real case, concentration changes also have to be taken into account.

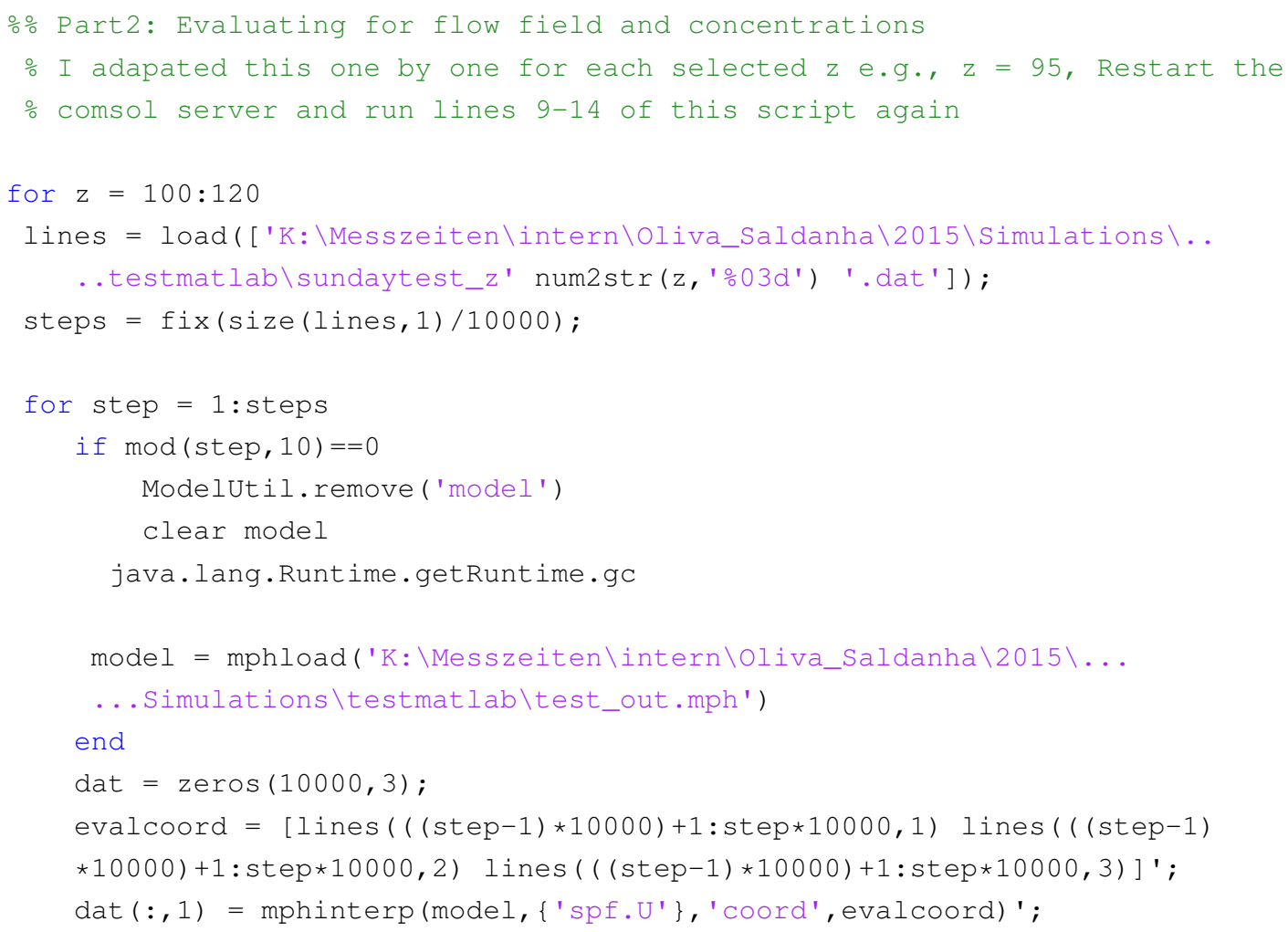




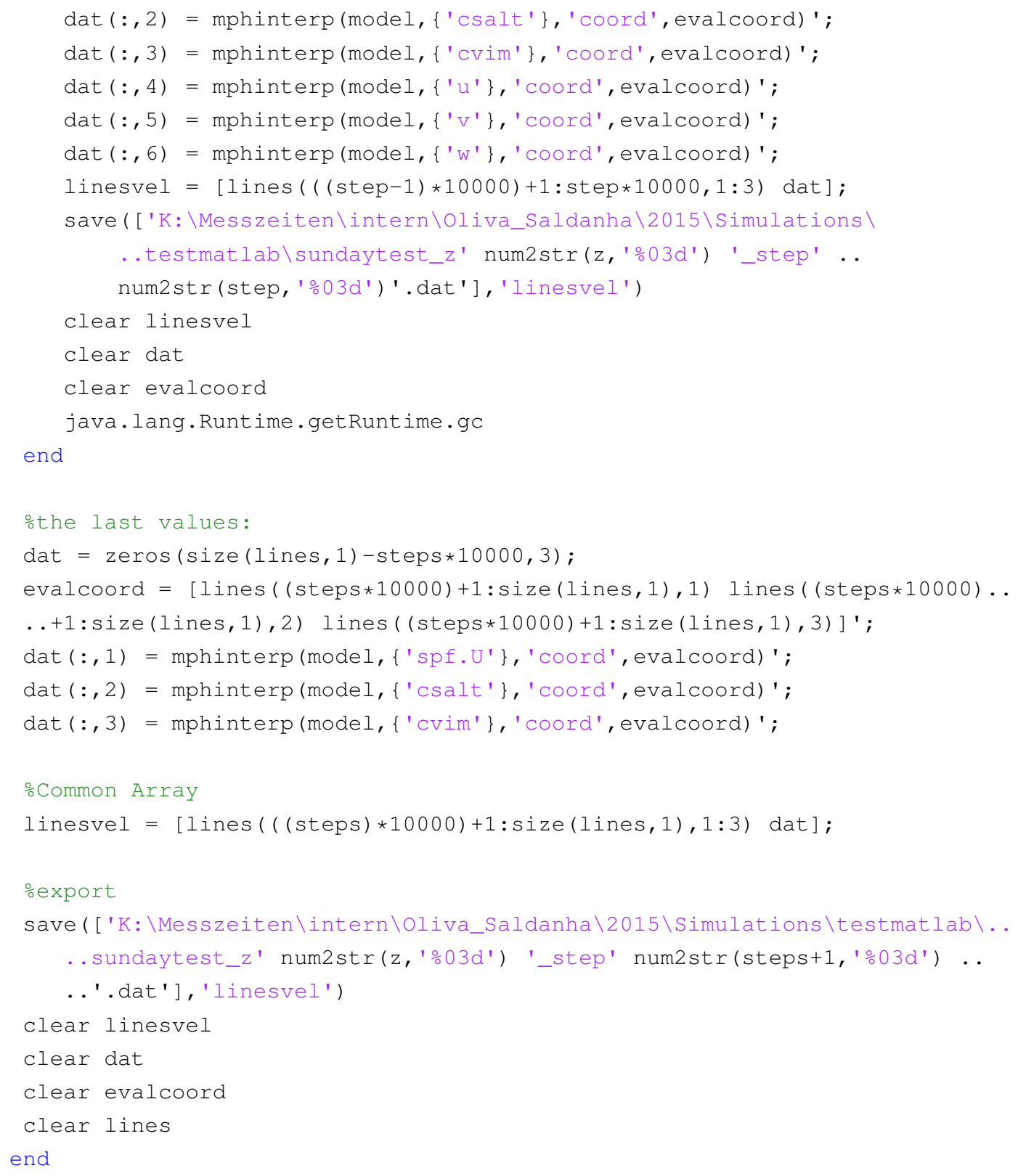




\section{Part3: Integration Along the Streamline}

After obtaining the values for the flow fiels and the oncentraion, assembly times of vimentin along the streamlines can be calculated in this third step. This is achieved by intgrating over the inverse velocity along the streamline for a changing concentration. Once the values are obtained from the second step, the integration can be done in MATLAB exclusively.

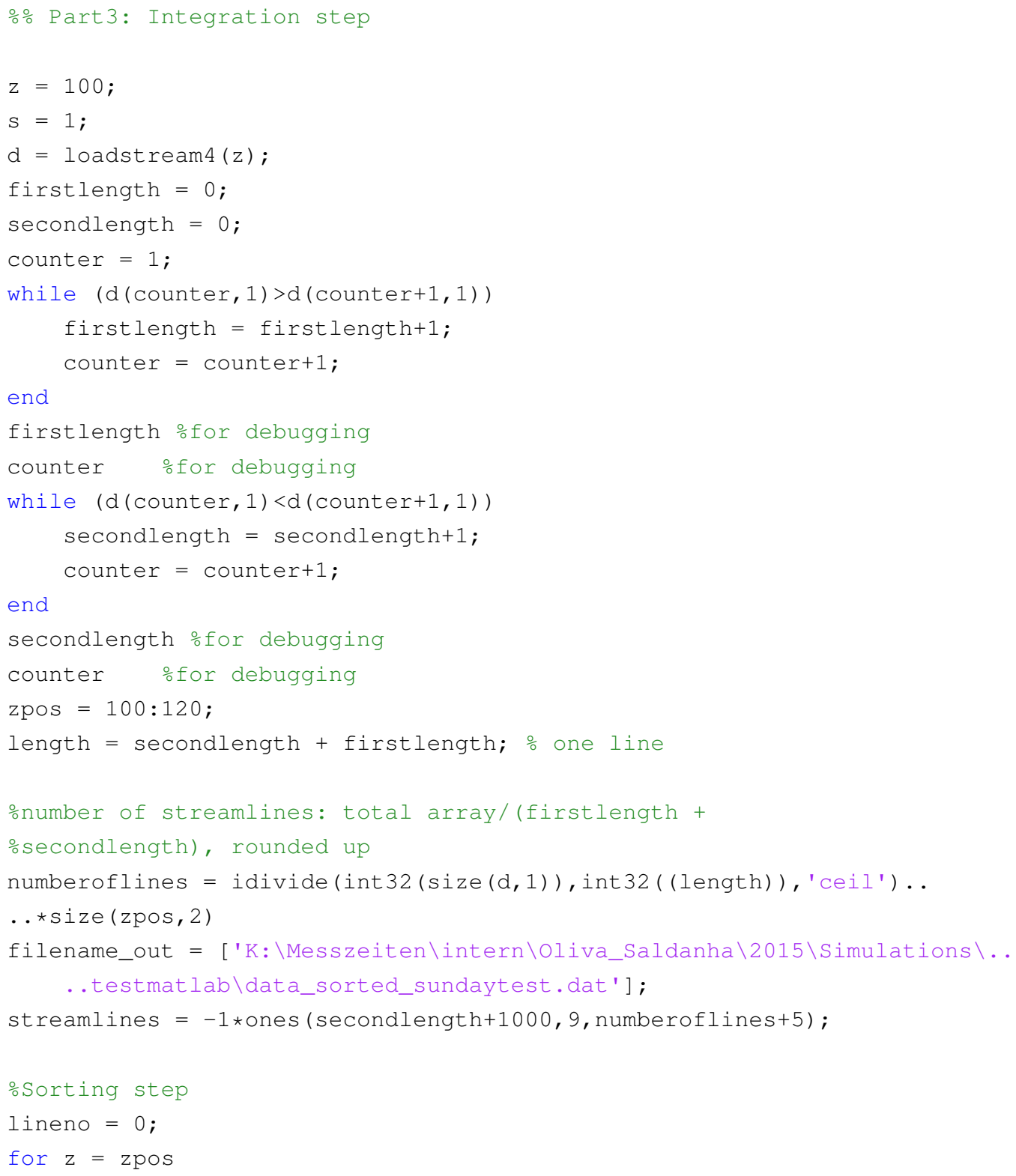




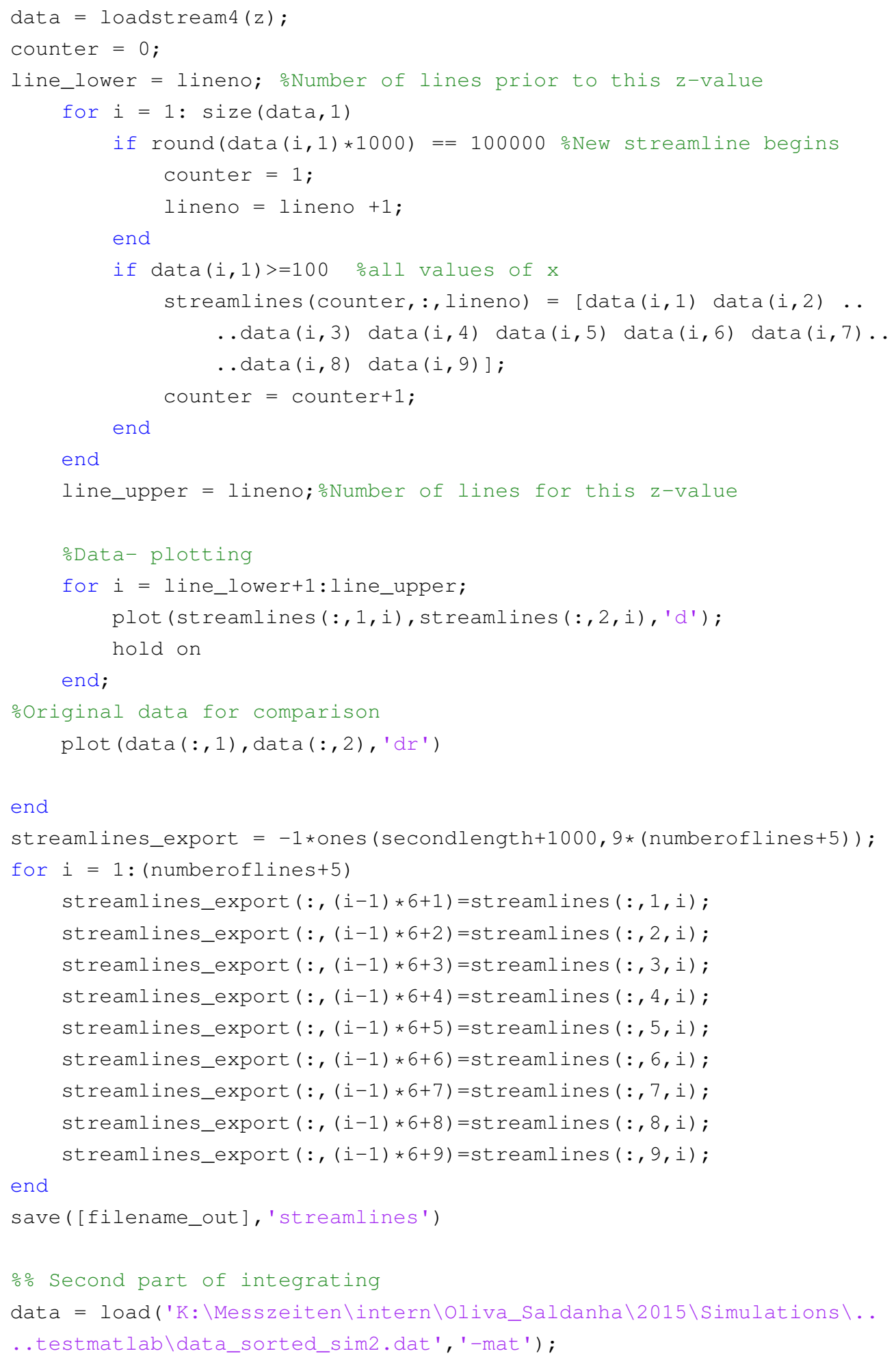




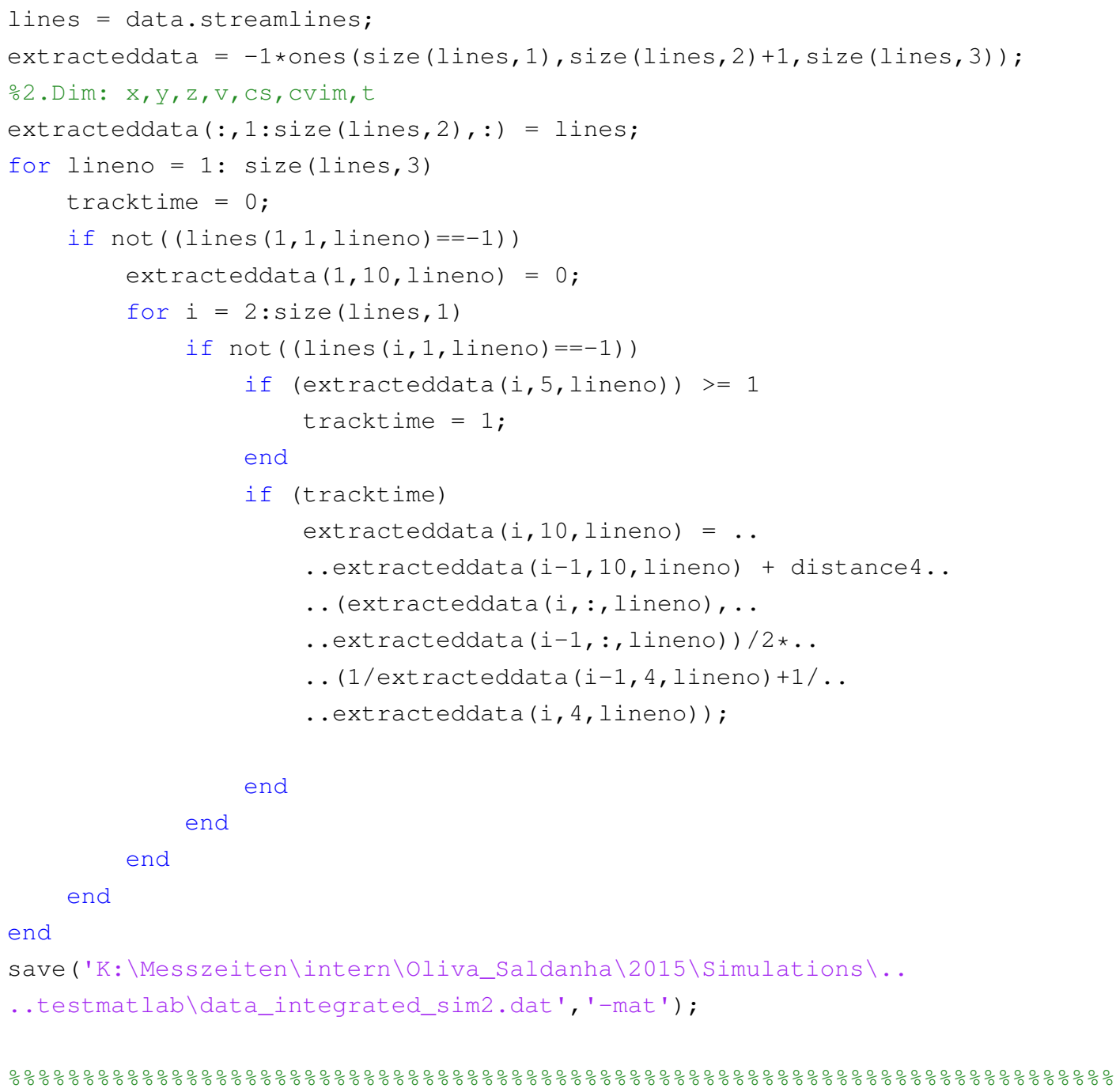

\section{Part4}

In the fourth step, we determine the ensembles that are calculated from the input data of COMSOL. From these calculated values, we can get arrays of each position along the streamline for the assembly times of vimentin.

응art 4: Determination of the ensembles

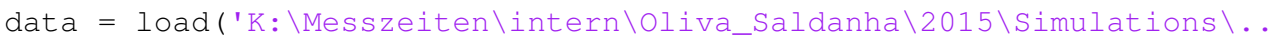

..testmatlab ldata_integrated_sim2.dat ', '-mat' ) ;

values = data.extracteddata; 


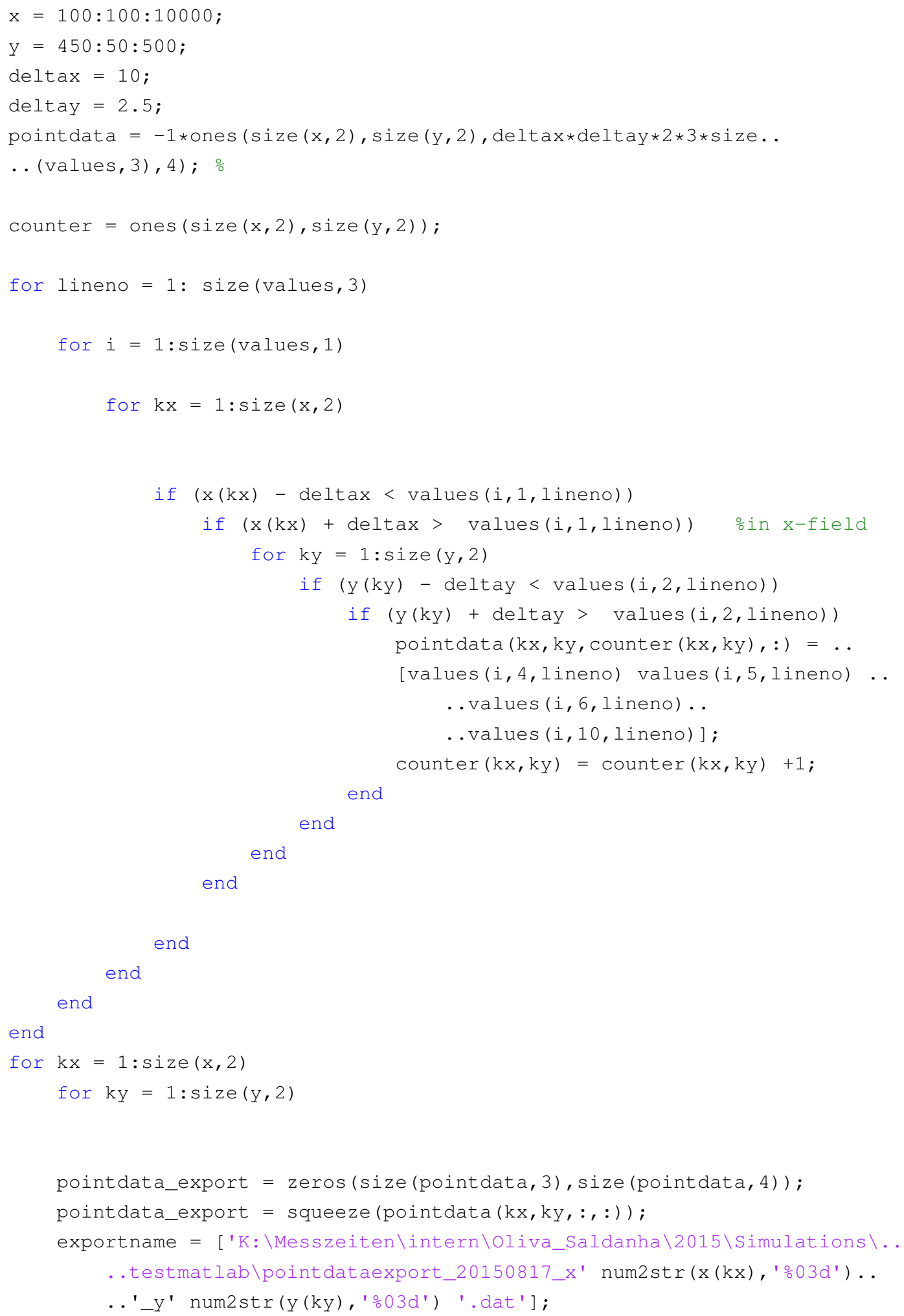


save (exportname, 'pointdata_export', '-ascii', '-tabs');

end

end

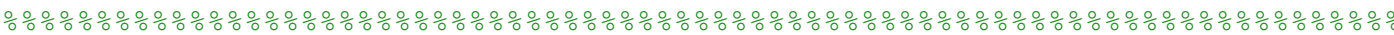

\section{Part5}

In the final step, we average the values obtained from the calculation to get a time evolution along the streamline under consideration and plot it as a function of length. The output data obtained can be exported to plotting softwares in readable formats like '.dat'.

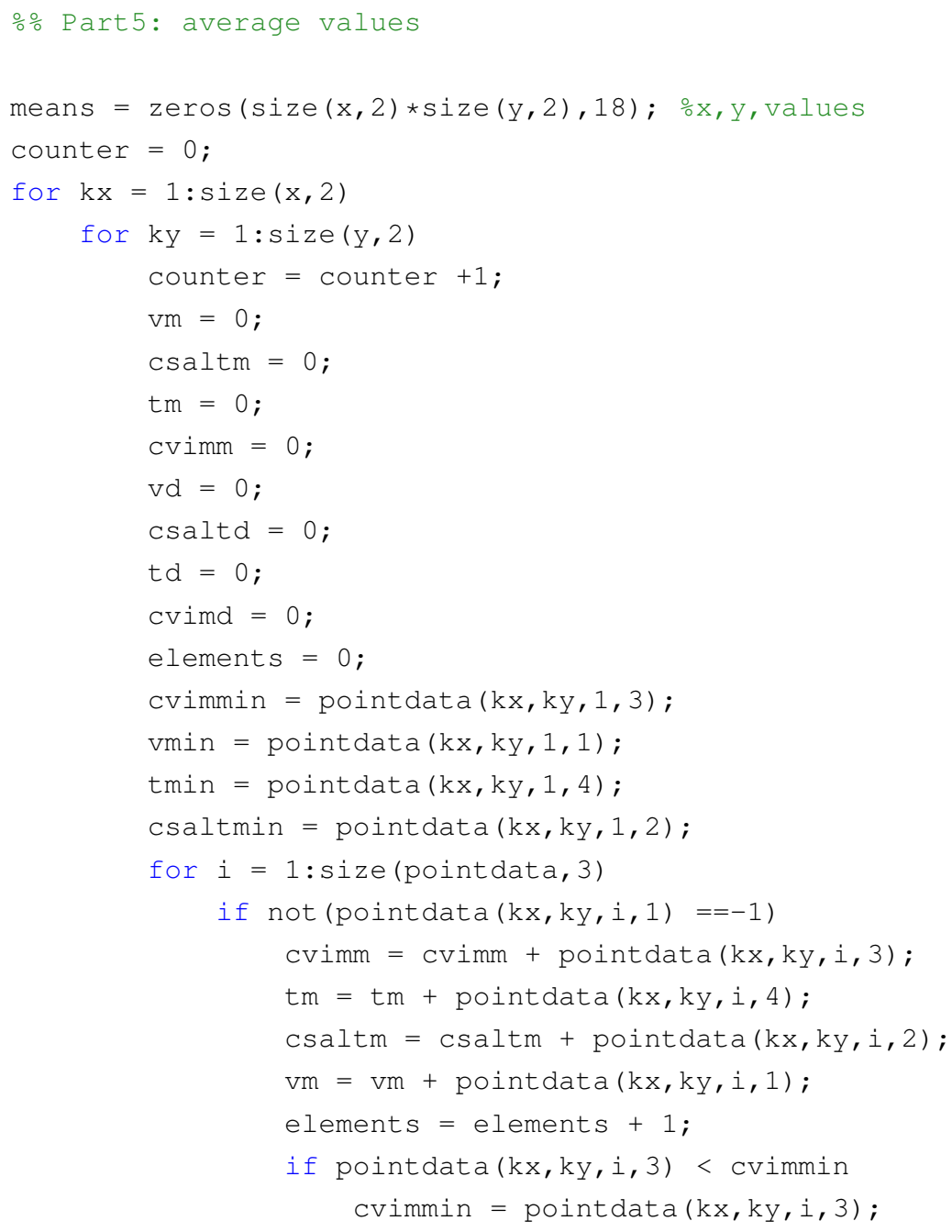




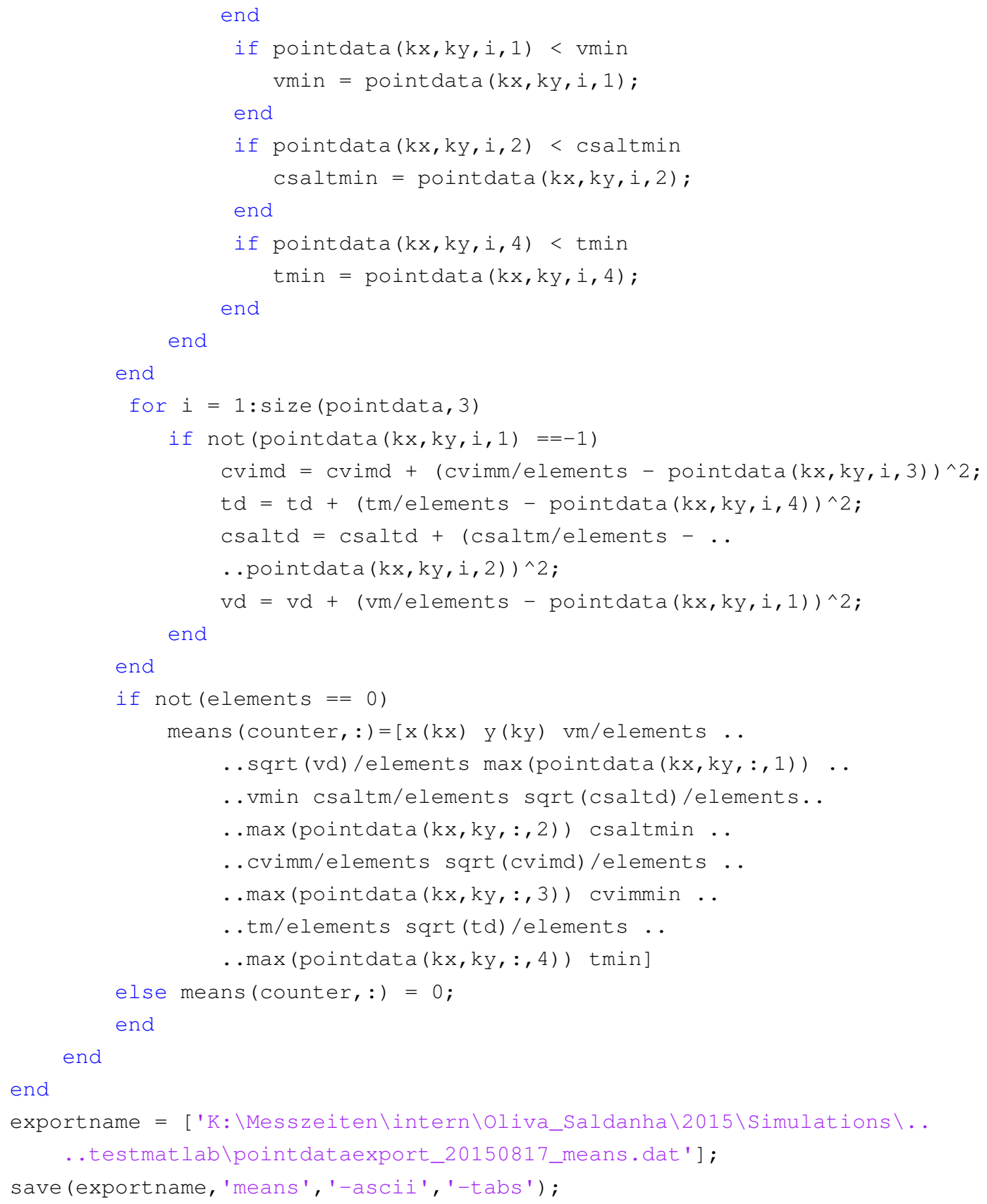




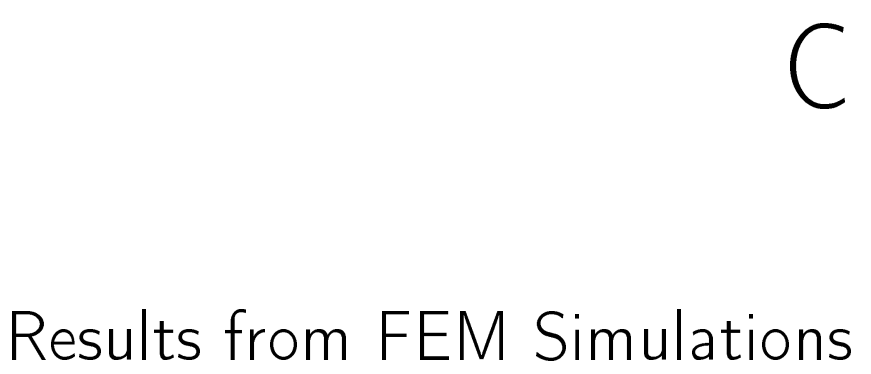

In Chapter. 3, we show the two-dimensional sections of the FEM simulations for flow rates 80:40. For the sake of completeness, we present the results from simulations for other flow rates 40:20 and 160:80 in this section. In order to achieve a better understanding of the flow conditions, a similar layout of the " $x-y$ " plane is presented. 
a)

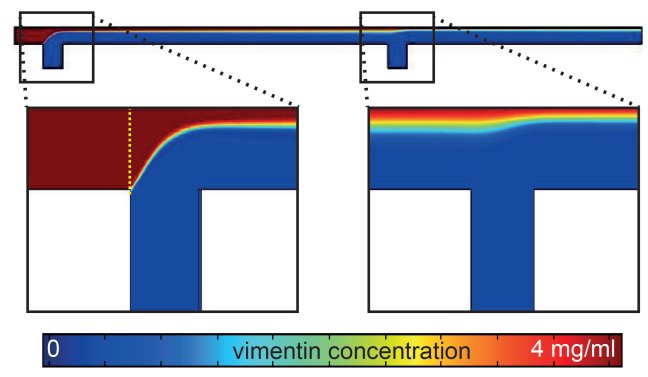

c)

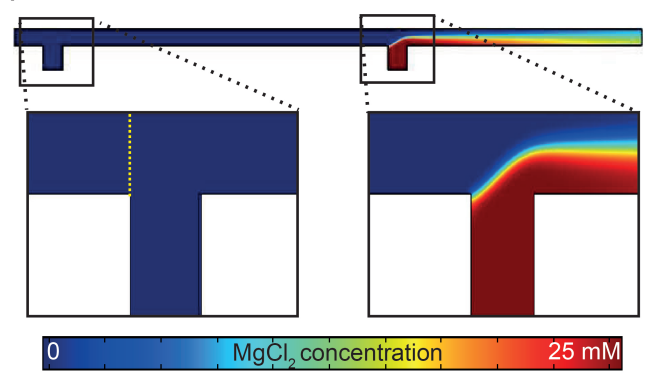

b)

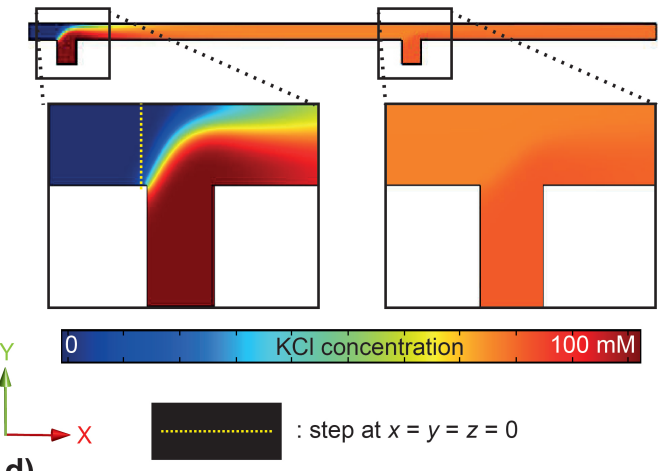

d)

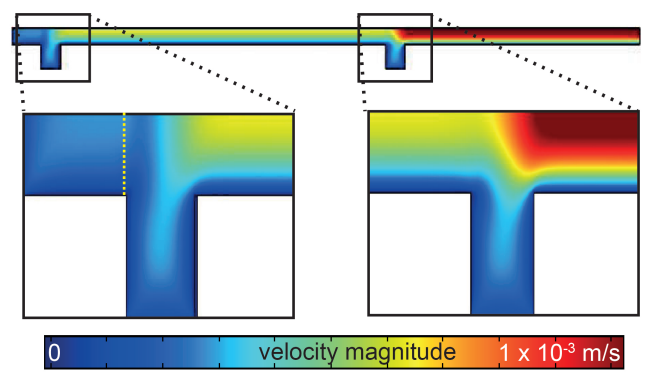

Figure C.1.: FEM simulations of the flow conditions and local concentrations in the microfluidic devices for flow rates 40:20. Simulations shown here are for a vimentin flow speed (central inlet) $20 \mu \mathrm{L} \mathrm{h}^{-1}$ and salt buffer flow speeds for each lateral inlet $40 \mu \mathrm{L} \mathrm{h}^{-1}$. The mid-plane $(x-y)$ is shown. (a) Vimentin concentration in the device. Enlarged are parts of the inflow of $\mathrm{KCl}$ (first cross) and $\mathrm{MgCl}_{2}$ (second cross). The vimentin concentration remains high in the central part since the large proteins diffuse slowly. (b) Change in $\mathrm{KCl}$ concentration. $\mathrm{KCl}$ is injected at the first cross, diffuses into the central protein stream and remains at a constant concentration when $\mathrm{MgCl}_{2}$ is injected. (c) Change in $\mathrm{MgCl}_{2}$ concentration. (d) Velocity magnitude. 
a)

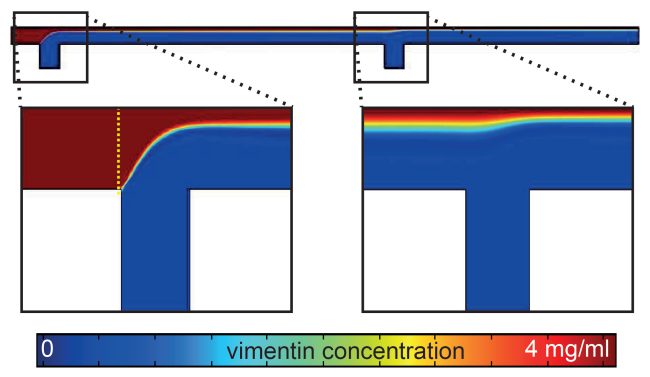

c)

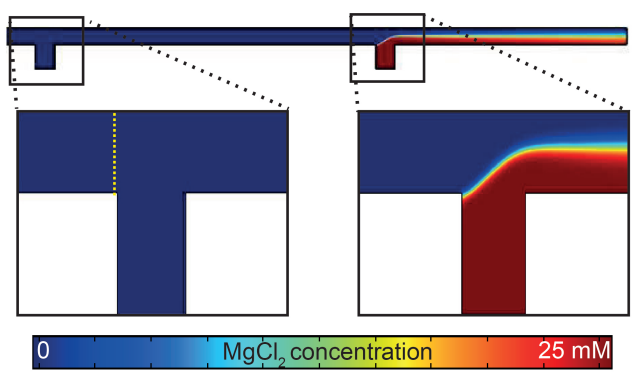

b)

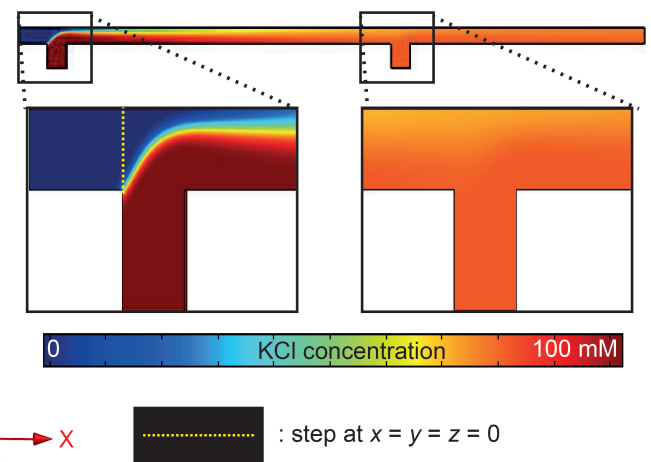

d)

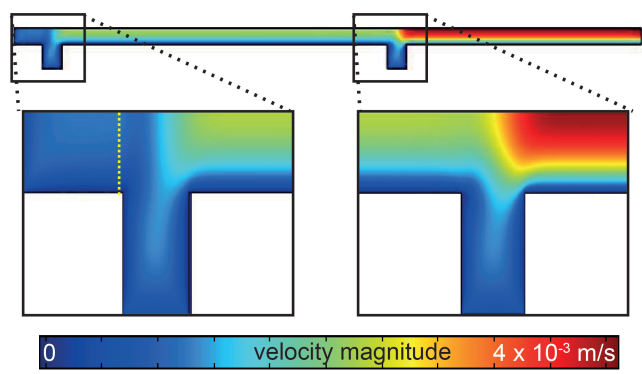

Figure C.2.: FEM simulations of the flow conditions and local concentrations in the microfluidic devices for flow rates 160:80. Simulations shown here are for a vimentin flow speed (central inlet) of $80 \mu \mathrm{L} \mathrm{h}^{-1}$ and salt buffer flow speeds for each lateral inlet of $160 \mu \mathrm{L}$ $\mathrm{h}^{-1}$. The mid-plane $(x-y)$ is shown. (a) Vimentin concentration in the device. Enlarged are parts of the inflow of $\mathrm{KCl}$ (first cross) and $\mathrm{MgCl}_{2}$ (second cross). The vimentin concentration remains high in the central part since the large proteins diffuse slowly. (b) Change in $\mathrm{KCl}$ concentration. $\mathrm{KCl}$ is injected at the first cross, diffuses into the central protein stream and remains at a constant concentration when $\mathrm{MgCl}_{2}$ is injected. (c) Change in $\mathrm{MgCl}_{2}$ concentration. (d) Velocity magnitude. 



\section{D}

\section{Kinetic Equations for Fitting Light Scattering}

Data

The modeling of the light scattering data is done in collaboration with Prof. Dr. Klaus Huber and Dr. Carlos G. Lopez, University of Paderborn, Germany.

In the works of Murphy et al. [125, 126], kinetic equations for a mathematical model of aggregation kinetics of Amyloid- $\beta$ has been derived which also explains several stages of fibrillogenesis from smaller subunits to mature fibrils. They consider a more complicated scheme, which reduces to our model when only the last two steps are considered. This is equivalent to setting to zero all reaction constants except $k_{1 a}$ and $k_{i, j}$ in reference [126]. Further, we consider the reaction order in step I to be equal to $p$, which corresponds to setting $p=q$ in Murphy and Pallitto's model. The equations describing the time dependence of the concentration of non-laterally aggregated species $f_{i}$ and laterally aggregated species $F_{i}$, where the subscript refers to the longitudinal degree of polymerisation (i.e. the number of length increments of $60 \mathrm{~nm}$ corresponding to the length of a tetramer) are given by: 


$$
\begin{gathered}
\frac{d f_{i}}{d t}=-p k_{n}\left[f_{i}\right]\left(\sum_{j=1}^{\infty}\left[f_{j}\right]\right)^{p-1} \\
\frac{d F_{i}}{d t}=+k_{n}\left[f_{i}\right]\left(\sum_{j=1}^{\infty}\left[f_{j}\right]\right)^{p-1}-\sum_{j=1}^{\infty} k_{i, j}\left[F_{i}\right]\left[F_{j}\right]+\frac{1}{2} \sum_{j=1}^{i-1} k_{j, i-j}\left[F_{j}\right]\left[F_{i-j}\right]
\end{gathered}
$$

If a polydisperse distribution of the species ' $\mathrm{f}$ ' is assumed, as will be done in scenario b) introduced below, the first term in Eqn. D.2 must be interpreted carefully. Since ' $\mathrm{f}$ ' type oligomers of any length can laterally aggregate with others of different size, the laterally aggregated bundles of $p$ oligomers (which account for the first term in Eqn. D.2 will have a heterogeneous mass per until length along a single bundle. Thus, $\left[F_{1}\right]$ refers to the concentration of filaments which contain at least one oligomer $f_{1}$, set by the prefactor $\left[f_{1}\right]$ in the first term. In order to calculate the length of the newly generated laterally aggregated species, we assume that the number average length is equal to that of the ' $f$ ' species. The nature of the ' $\mathrm{f}$ ' species is adjusted to the measured values of $M_{w}$ and $R_{g}$ (see Figure 1 of the manuscript) for the sample selected. The smallest soluble vimentin unit is the tetramer, with $L=60 \mathrm{~nm}$ and $M_{w}=214000 \mathrm{~g} / \mathrm{mol}$. Given these are values commensurate with the observed $R_{g}$ and $M_{w}$ values, the discrete nature of vimentin should be considered for the evaluation of the initial state of the respective kinetic run. Since we do not have access to the complete distribution of species, which is likely to consist of multifolds of tetramers both in the lateral and longitudinal directions, we consider here two different scenarios to fix the initial state of our kinetic model:

a) a bimodal population consisting of 1) tetramers and 2) an oligomeric ULF (p $=6$ ) with a longitudinal degree of polymerisation of $n_{l}$.

b) a population of longitudinal multifold s of the tetramer following a SZ distribution and a population of monomeric ULFs $(p=6)$.

Scenarios a) and b) are capable of reproducing the $R_{g}$ vs. $M_{w}$ results as shown in Chapter. 5. For scenario a), the number fraction of oligomeric ULFs is approximately constant at $1 \%$ and the variation in $M_{w}$ and $R_{g}$ is caused by the degree of longitudinal aggregation of ULFs. For scenario b), the variation in $R_{g}$ and $M_{w}$ is the result of an increase in $L_{w}$ of the longitudinal tetrameric multifolds. The effective cross sectional diameter of tetramers and longitudinal multifolds thereof is taken to be $d_{\text {tet }}=5 \mathrm{~nm}$. The diameter of ULF is coarsely approximated at $d_{F}=\sqrt{p} d_{t e t}$. 
The static and kinetic data can be explained using scenario a) or b), with no significant difference in the agreement between model and experiment. The fit parameters $k_{n}$ and $\delta \omega$ obtained for a) and b) do however vary significantly ( $\simeq$ $\times 2$ ). The data presented in the work is modelled assuming scenario a). They are compared with results from model fits based on scenario $b$ ).

The end-to-end aggregation constant $k_{i, j}$ is given by:

$$
\begin{array}{r}
k_{i, j}=\frac{k_{B} T N_{A}(\delta \omega)^{2}}{3 \eta} \frac{1}{L_{i}+L_{j}}\left(\frac{\ln \left(L_{i} / d\right)+0.312+0.565 \frac{d}{L_{i}}-0.1 \frac{d^{2}}{L_{i}^{2}}}{L_{i}}\right. \\
\left.+\frac{\ln \left(L_{i} / d\right)+0.312+0.565 \frac{d}{L_{j}}-0.1 \frac{d^{2}}{L_{j}^{2}}}{L_{j}}\right)
\end{array}
$$

which for simplicity was reduced in the model of Pallitto and Murphy[126] to:

$$
\overline{k_{i, j}}=\frac{k_{B} T N_{A}(\delta \omega)^{2}}{3 \eta} \frac{1}{\bar{L}}\left(\frac{\ln (\bar{L} / d)+0.312+0.565 \frac{d}{\bar{L}}-0.1 \frac{d^{2}}{\bar{L}^{2}}}{\bar{L}}\right)
$$

where $\bar{L}$ is the number average length of the $F$ species. Eqn. D.4 can be further approximated as:

$$
k_{i, j}=\frac{k_{B} T N_{A}(\delta \omega)^{2}}{3 \eta} \frac{A}{\bar{L}^{2+\mu}}=G \frac{1}{L^{2+\mu}}
$$

where $A$ and $\mu$ are parameters set so that $\frac{A}{\bar{L}^{2+\mu}}$ closely matches the expression in big brackets in Eqn. D.4. For our experiments, where $L / d$ does not vary by more that an order of magnitude, using this approximation never leads to an error in $k_{i, j}$ greater than $\simeq 3 \%$

The nth moment of the concentration of the two species are defined as:

$$
\lambda_{f n}=\sum_{i=0}^{\infty} i^{n}\left[f_{i}\right] \quad \text { and } \quad \lambda_{F n}=\sum_{i=0}^{\infty} i^{n}\left[F_{i}\right]
$$

The equations describing the zeroth to second moments of the concentration of the initial species (subscript $f$ ) and the laterally aggregated species (subscript $F$ ) are given as:

$$
\frac{d \lambda_{f 0}}{d t}=-p k_{n} \lambda_{f 0}^{p}
$$




$$
\begin{gathered}
\frac{d \lambda_{f 1}}{d t}=-p k_{n} \lambda_{f 1} \lambda_{f 0}^{p-1} \\
\frac{d \lambda_{f 2}}{d t}=-p k_{n} \lambda_{f 2} \lambda_{f 0}^{p-1} \\
\frac{d \lambda_{F 0}}{d t}=k_{n} \lambda_{f 0}^{p}-\frac{1}{2} \overline{k_{i p, j p}} \lambda_{F 0}^{2} \\
\frac{d \lambda_{F 1}}{d t}=p k_{n} \lambda_{f 1} \lambda_{f 0}^{p-1} \\
\frac{d \lambda_{F 2}}{d t}=p^{2} k_{n} \lambda_{f 2} \lambda_{f 0}^{p-1}+\overline{k_{i p, j p}} \lambda_{F 1}^{2}
\end{gathered}
$$

The initial conditions are given by:

$$
\begin{gathered}
\lambda_{f 0}(0)=\left[M_{f}\right] \frac{M_{t e t}}{M_{f, n}(0)} \\
\lambda_{f 1}(0)=\left[M_{f}\right] \\
\lambda_{f 2}(0)=\left[M_{f}\right] \frac{M_{f, w}(0)}{M_{t e t}}
\end{gathered}
$$

where $\left[M_{f}\right]$ is the total concentration of vimentin in ' $\mathrm{f}$ ' species expressed as (number of non laterally aggregated tetramers)/L, $M_{\text {tet }}$ is the molar mass of a tetramer, $M_{f, n}(0)$ and $M_{f, w}(0)$ are the number and weight averaged molecular weights of the ' $f$ ' species at time zero respectively. If a monodisperse population of tetramers is assumed as the initial condition for the species ' $\mathrm{f}$ ', see scenario a), then $M_{f, n}(0)$ $=M_{f, w}(0)=M_{t e t}$ and $\lambda_{f 0}(0)=\lambda_{f 1}(0)=\lambda_{f 2}(0)=\left[M_{f}\right]$. The starting conditions for $\lambda_{F 0}(0), \lambda_{F 1}(0)$ and $\lambda_{F 2}(0)$ are calculated in an analogous manner:

$$
\begin{gathered}
\lambda_{F 0}(0)=\frac{\left[M_{F}\right]}{p n_{l}} \\
\lambda_{F 1}(0)=\left[M_{F}\right] \\
\lambda_{F 2}(0)=\left[M_{F}\right] p n_{l}
\end{gathered}
$$


where $\left[M_{F}\right]$ is the total concentration of vimentin in ' $\mathrm{F}^{\prime}$ species expressed as (number of laterally aggregated tetramers)/L and $n_{l}$ is the longitudinal degree of polymerisation of the filaments F. For scenario $b$ ), $n_{l}$ is equal to 1 .

$M_{w}$ for the entire ensemble is calculated from:

$$
M_{w}=\frac{\lambda_{F 2}+\lambda_{f 2}}{\lambda_{F 1}+\lambda_{f 1}} M_{t e t}
$$

which allows us to fit $\mathrm{Mw}$ data obtained from light scattering experiments with the above model.

In order to be able to also fit $\left\langle R_{g}^{2}\right\rangle_{z}$, we assume that the filaments of species $\mathrm{F}$ are distributed according to the Schultz-Zimm formula (Eqn. 5.16). The polydispersities for the two populations are calculated using:

$$
p . d_{. \alpha}=\frac{\lambda_{\alpha 2} \lambda_{\alpha 0}}{\lambda_{\alpha 1}^{2}}
$$

where $\alpha$ can be equal to $f$ or $F$. Once the polydispersity is known, the $z$ averaged molecular mass $M_{z}$ for each population can be calculated according to Eqn. 5.10 The $z$-averaged squared radii of gyration for the $\mathrm{f}$ and F species $R_{g, f}^{2}$ and $R_{g, F}^{2}$ respectively can be calculated from:

$$
\begin{gathered}
R_{g, f}^{2}=\left(\frac{M_{z, f}}{12 M_{L} / p}\right)^{2} \\
R_{g, F}^{2}=\left(\frac{M_{z, F}}{12 M_{L}}\right)^{2}
\end{gathered}
$$

where $M_{L}$ is the mass per unit length of the $F$ species. We note that $R_{g, f}^{2}$ is independent of time. The $z$-averaged square radius of gyration for the entire ensemble, which is measured by static light scattering is given by:

$$
\left\langle R_{g}^{2}\right\rangle_{z}=\frac{\lambda_{F 2} R_{g, F}^{2}+\lambda_{f 2} R_{g, f}^{2}}{\lambda_{f 2}+\lambda_{F 2}}
$$

The $z$-averaged diffusion coefficient for the $\mathrm{f}$ species $D_{f, z}$ and for the $\mathrm{F}$ species $D_{F, z}$ can be calculated by Eqn. 5.20 using the weight averaged rod lengths for each species $L_{f, w}$ and $L_{F, w}$ respectively. The $z$-averaged diffusion coefficient for the full ensemble can calculated as:

$$
D_{z}=\frac{\lambda_{f 2} D_{f, z}+\lambda_{F 2} D_{F, z}}{\lambda_{f, 2}+\lambda_{F, 2}}
$$




\section{\begin{tabular}{l|l} 
Appendix & KINETIC EQUATIONS FOR FITTING LIGHT SCATTERING DATA
\end{tabular}}

The above equations cannot be solved analytically for the general case $k_{n} \neq 0$ and $k_{i, j} \neq 0$. For the special case where end-to-end elongation does not take place $\left(k_{i, j}=0\right)$, the above equations can be solved to give:

$$
\begin{gathered}
\lambda_{f 0}=\left(\lambda_{f 0}(0)^{-p}\left[k_{n} p(p-1) t \lambda_{f 0}(0)^{p}+\lambda_{f 0}(0)\right]\right)^{\frac{1}{1-p}} \\
\lambda_{f 1}=\left(\lambda_{f 1}(0)^{-p}\left[k_{n} p(p-1) \lambda_{f 0}(0)^{p-1} \lambda_{f 1}(0) t+\lambda_{f 1}(0)\right]\right)^{\frac{1}{1-p}} \\
\lambda_{f 2}=\left(\lambda_{f 2}(0)^{-p}\left[k_{n} p(p-1) \lambda_{f 0}(0)^{p-1} \lambda_{f 2}(0) t+\lambda_{f 2}(0)\right]\right)^{\frac{1}{1-p}} \\
\lambda_{F 0}=\left(\lambda_{f 0}(0)-\lambda_{f}\right) / p+\lambda_{F 0}(0) \\
\lambda_{F 1}=\lambda_{f 0}(0)-\lambda_{f}+\lambda_{F 1}(0) \\
\lambda_{F 2}=\left(\lambda_{f 0}(0)-\lambda_{f}\right) p+\lambda_{F 2}(0)
\end{gathered}
$$

We can also obtain an analytical solution when $k_{n}=0$. The initial conditions have to be changed to reflect the initial population of laterally aggregated fibres. The analytical expressions for $\lambda_{F 0}, \lambda_{F 1}$ and $\lambda_{F 2}$ are then given by:

$$
\begin{gathered}
\lambda_{F 0}=\left[\left(\frac{M_{L}}{M_{U L F} \lambda_{F 1}}\right)^{2+\mu} \frac{G}{2}(\mu+3) t+\lambda_{F 0}(0)^{-(\mu+3)}\right]^{-1 /(\mu+3)} \\
\lambda_{F 1}=\lambda_{F 1}(0) \\
\lambda_{F 2}=\lambda_{F 2}(0)+H\left((J+I t)^{m+1}-J^{m+1}\right) /(I(m+1))
\end{gathered}
$$

where

$$
\begin{gathered}
m=-\frac{2+\mu}{3+\mu} \\
H=G \lambda_{F 2}(0)^{-\mu} M_{L}^{2+\mu} \\
I=\left(M_{L} /\left(M_{U L F} L_{F 1}(0)\right)\right)^{2+\mu} G(\mu / 2+1.5) \\
J=L_{F 0}(0)^{-\mu-3}
\end{gathered}
$$




$$
G=\frac{k_{B} T N_{A}}{3 \eta} A(\delta \omega)^{2}
$$

In order to fit data, we solve Eqn. D.6 D.11 and D.5 numerically using a Runge Kutta 4 method implemented in Igor pro v6.36. The time step was varied depending on sample and fit parameters between $0.3 \mathrm{~ms}$ and $0.01 \mathrm{~ms}$. We use the values of $p$ and $k_{n}$ obtained from first fitting Eqn. D.12 D.17 as our starting guesses. The initial conditions are obtained from the values of $M_{w}$ and $\left\langle R_{g}^{2}\right\rangle_{z}$ measured for the sample prior to salt addition. We did not implement a least squares minimisation routine, rather, the parameters were adjusted by eye until they gave a good fit to the data. This typically took of the order of 20 iterations. 



\section{Bibliography}

[1] B. Alberts, A. Johnson, J. Lewis, M. Raff, K. Roberts, and P. Walter. Molecular Biology of the cell. Garland Science Taylor and Francis Group, New York NY, sixth edition, 2014. (cited on page4)

[2] H. Herrmann, H. Bär, L. Kreplak, S. V. Strelkov, and U. Aebi. Intermediate filaments: from cell architecture to nanomechanics. Nature Reviews. Molecular Cell Biology, 8(7):562-573, 2007. (cited on page 4.5. and 7

[3] D. A. Fletcher and R. D. Mullins. Cell mechanics and the cytoskeleton. Nature, 463(7280):485-492, 2010. (cited on page 4 )

[4] B. Wickstead and K. Gull. The evolution of the cytoskeleton. The Journal of Cell Biology, 194(4):513-525, 2011. (cited on page 4)

[5] N. Stuurman, S. Heins, and U. Aebi. Nuclear lamins: their structure, assembly, and interactions. Journal of Structural Biology, 122(1-2):42-66, 1998. (cited on page 4 )

[6] H. Herrmann, L. Kreplak, and U. Aebi. Isolation, characterization, and in vitro assembly of intermediate filaments. In M. B. Omary and P. A. Coulombe, editors, Intermediate Filament Cytoskeleton, volume 78 of Methods in Cell Biology, pages 3 - 24. Academic Press, 2004. (cited on page 4 10. and 11

[7] E. Fuchs and K. Weber. Intermediate filaments: structure, dynamics, function, and disease. Annual Review of Biochemistry, 63:345-382, 1994. (cited on page 5,7 , and 8

[8] E. Fuchs. A structural scaffolding of intermediate filaments in health and disease. Science, 279(5350):514-519, 1998. (cited on page6) 
[9] C.-H. Lee, M.-S. Kim, B. M. Chung, D. J. Leahy, and P. A. Coulombe. Structural basis for heteromeric assembly and perinuclear organization of keratin filaments. Nature Structural \& Molecular Biology, 19(7):707-715, 2012. (cited on page 6

[10] P. M. Steinert, Y. H. Chou, V. Prahlad, D. A. D. Parry, L. N. Marekov, K. C. $\mathrm{Wu}, \mathrm{S}$. I. Jang, and R. D. Goldman. A high molecular weight intermediate filament-associated protein in BHK-21 Cells Is Nestin, a Type VI Intermediate Filament Protein. Journal of Biological Chemistry, 274(14):9881-9890, 1999. (cited on page 6)

[11] J. I. Satoh, T. Yamamura, and K. Arima. The 14-3-3 protein $\epsilon$ isoform expressed in reactive astrocytes in demyelinating lesions of multiple sclerosis binds to vimentin and glial fibrillary acidic protein in cultured human astrocytes. The American Journal of Pathology, 165(2):577-592, 2004. (cited on page 6

[12] H. M. Evans, E. Surenjav, C. Priest, S. Herminghaus, R. Seemann, and T. Pfohl. In situ formation, manipulation, and imaging of dropletencapsulated fibrin networks. Lab on a Chip, 9(13):1933-1941, 2009. (cited on page 7

[13] S. Kim and P. A. Coulombe. Intermediate filament scaffolds fulfill mechanical, organizational, and signaling functions in the cytoplasm. Genes \& Development, 21(13):1581-1597, 2007. (cited on page7

[14] D. M. Toivola, P. Strnad, A. Habtezion, and M. B. Omary. Intermediate filaments take the heat as stress proteins. Trends in Cell Biology, 20(2):7991, 2010. (cited on page 7)

[15] H. Herrmann, S. V. Strelkov, P. Burkhard, and U. Aebi. Intermediate filaments: primary determinants of cell architecture and plasticity. The Journal of Clinical Investigation, 119(7):1772-1783, 2009. (cited on page 7)

[16] L. Chang and R. D. Goldman. Intermediate filaments mediate cytoskeletal crosstalk. Nature Reviews. Molecular Cell Biology, 5(8):601-613, 2004. (cited on page 7

[17] D. Kong, Y. Li, Z. Wang, and F. H. Sarkar. Cancer stem cells and epithelial-tomesenchymal transition (emt)-phenotypic cells: are they cousins or twins? Cancers, 3(1):716-729, 2011. (cited on page 7 ) 
[18] M. Guarino, A. Tosoni, and M. Nebuloni. Direct contribution of epithelium to organ fibrosis: epithelial-mesenchymal transition. Human Pathology, 40(10):1365-1376, 2009. (cited on page7)

[19] J. H. Tsai and J. Yang. Epithelial-mesenchymal plasticity in carcinoma metastasis. Genes \& Development, 27(20):2192-2206, 2013. (cited on page7)

[20] J. M. Dave and K. J. Bayless. Vimentin as an integral regulator of cell adhesion and endothelial sprouting. Microcirculation (New York, N.Y. : 1994), 21(4):333-344, 2014. (cited on page 7 )

[21] M. G. Mendez, S. I. Kojima, and R. D. Goldman. Vimentin induces changes in cell shape, motility, and adhesion during the epithelial to mesenchymal transition. The federation of american societies for experimental biology (FASEB) Journal, 24(6):1838-1851, 2010. (cited on page 7)

[22] P. A. Janmey, U. Euteneuer, P. Traub, and M. Schliwa. Viscoelastic properties of vimentin compared with other filamentous biopolymer networks. The Journal of Cell Biology, 113(1):155-160, 1991. (cited on page7)

[23] B. Eckes, D. Dogic, E. Colucci-Guyon, N. Wang, A. Maniotis, D. Ingber, A. Merckling, F. Langa, M. Aumailley, A. Delouvee, V. Koteliansky, C. Babinet, and T. Krieg. Impaired mechanical stability, migration and contractile capacity in vimentin-deficient fibroblasts. Journal of Cell Science, 111(13):1897-1907, 1998. (cited on page 8

[24] N. Wang and D. Stamenovic. Mechanics of vimentin intermediate filaments. Journal of Muscle Research and Cell Motility, 23(5-6):535-540, 2002. (cited on page 8

[25] T. Katsumoto, A. Mitsushima, and T. Kurimura. The role of the vimentin intermediate filaments in rat 3y1 cells elucidated by immunoelectron microscopy and computer-graphic reconstruction. Biology of the Cell, 68(2):139-146, 1990. (cited on page 8)

[26] R. D. Goldman, S. Khuon, Y. H. Chou, P. Opal, and P. M. Steinert. The function of intermediate filaments in cell shape and cytoskeletal integrity. The Journal of Cell Biology, 134(4):971-983, 1996. (cited on page 8 
[27] S. Portet, N. Mücke, R. Kirmse, J. Langowski, M. Beil, and H. Herrmann. Vimentin intermediate filament formation: In vitro measurement and mathematical modeling of the filament length distribution during assembly. Langmuir, 25(15):8817-8823, 2009. (cited on page 8, 97 and 107.

[28] L. Chang, Y. Shav-Tal, T. Trcek, R. H. Singer, and R. D. Goldman. Assembling an intermediate filament network by dynamic cotranslation. The Journal of Cell Biology, 172(5):747-758, 2006. (cited on page 8 )

[29] G. Colakoğlu and A. Brown. Intermediate filaments exchange subunits along their length and elongate by end-to-end annealing. The Journal of Cell Biology, 185(5):769-777, 2009. (cited on page 8 and 10

[30] E. Czeizler, A. Mizera, E. Czeizler, R. J. Back, J. E. Eriksson, and I. Petre. Quantitative analysis of the self-assembly strategies of intermediate filaments from tetrameric vimentin. IEEE/ACM Transactions on Computational Biology and Bioinformatics, 9(3):885-898, 2012. (cited on page 8)

[31] R. Kirmse, S. Portet, N. Mücke, U. Aebi, H. Herrmann, and J. Langowski. A quantitative kinetic model for the in vitro assembly of intermediate filaments from tetrameric vimentin. The Journal of Biological Chemistry, 282(25):18563-18572, 2007. (cited on page 8, 13, and 107

[32] S. Winheim, A. R. Hieb, M. Silbermann, E. M. Surmann, T. Wedig, H. Herrmann, J. Langowski, and N. Mücke. Deconstructing the late phase of vimentin assembly by total internal reflection fluorescence microscopy (tirfm). PloS one, 6(4):e19202, 2011. (cited on page 8

[33] B. Nöding and S. Köster. Intermediate filaments in small configuration spaces. Physical Review Letters, 108(8), 2012. (cited on page 8 and 83)

[34] H. Herrmann, M. Häner, M. Brettel, S. A. Müller, K. N. Goldie, B. Fedtke, A. Lustig, W. W. Franke, and U. Aebi. Structure and assembly properties of the intermediate filament protein vimentin: the role of its head, rod and tail domains. Journal of Molecular Biology, 264(5):933 - 953, 1996. (cited on page 8. 11, $13,35,63,100$ and 107

[35] M. E. Brennich, J.-F. Nolting, C. Dammann, B. Nöding, S. Bauch, H. Herrmann, T. Pfohl, and S. Köster. Dynamics of intermediate filament assem- 
bly followed in micro-flow by small angle x-ray scattering. Lab on a Chip, 4(11):708-716, 2011. (cited on page 8, 25, 28, 32, 58, 74, 75, 77, 83, and 107)

[36] H. Herrmann and U. Aebi. Intermediate filament assembly: fibrillogenesis is driven by decisive dimer-dimer interactions. Current Opinion in Structural Biology, 8(2):177-185, 1998. (cited on page 8)

[37] M. E. Brennich, S. Bauch, U. Vainio, T. Wedig, H. Herrmann, and S. Köster. Impact of ion valency on the assembly of vimentin studied by quantitative small angle x-ray scattering. Soft Matter, 10(12):2059-2068, 2014. (cited on page $9,10,14,62,63,64,76,77$, and 83.

[38] M. Kooijman, M. Bloemendal, P. Traub, R. van Grondelle, and H. van Amerongen. Transient electric birefringence study of intermediate filament formation from vimentin and glial fibrillary acidic protein. Journal of Biological Chemistry, 272(36):22548-22555, 1997. (cited on page 9, 10, $13,14$. and 62

[39] I. Hofmann, H. Herrmann, and W. W. Franke. Assembly and structure of calcium-induced thick vimentin filaments. European Journal of Cell Biology, 56(2):328-341, 1991. (cited on page 9, 14, 35, and 62.

[40] H. Herrmann, M. Häner, M. Brettel, N. O. Ku, and U. Aebi. Characterization of distinct early assembly units of different intermediate filament proteins. Journal of Molecular Biology, 286(5):1403 - 1420, 1999. (cited on page 10 and 11)

[41] K. L. Vikstrom, S. S. Lim, R. D. Goldman, and G. G. Borisy. Steady state dynamics of intermediate filament networks. The Journal of Cell Biology, 118(1):121-129, 1992. (cited on page10

[42] B. Nöding, H. Herrmann, and S. Köster. Direct observation of subunit exchange along mature vimentin intermediate filaments. Biophysical Journal, 107(12):2923-2931, 2014. (cited on page 10 and 83)

[43] O. I. Wagner, S. Rammensee, N. Korde, Q. Wen, J. F. Leterrier, and P. A. Janmey. Softness, strength and self-repair in intermediate filament networks. Experimental Cell Research, 313(10):2228-2235, 2007. (cited on page 10

[44] S. Köster, Y.-C. Lin, H. Herrmann, and D. A. Weitz. Nanomechanics of vimentin intermediate filament networks. Soft Matter, 6(9):1910, 2010. (cited on page 10,13 , and 65 , 
[45] Y.-C. Lin, C. P. Broedersz, A. C. Rowat, T. Wedig, H. Herrmann, F. C. Mackintosh, and D. A. Weitz. Divalent cations crosslink vimentin intermediate filament tail domains to regulate network mechanics. Journal of Molecular Biology, 399(4):637-644, 2010. (cited on page 10, 13,64 and 65

[46] Y. C. Lin, Norman Y. Yao, C. P. Broedersz, H. Herrmann, F. C. Mackintosh, and D. A. Weitz. Origins of elasticity in intermediate filament networks. Physical Review Letters, 104(5):58101, 2010. (cited on page 10 and 13)

[47] J. X. Tang, T. Ito, T. Tao, P. Traub, and P. A. Janmey. Opposite effects of electrostatics and steric exclusion on bundle formation by f-actin and other filamentous polyelectrolytes. Biochemistry, 36(41):12600-12607, 1997. (cited on page 10

[48] H. Herrmann, I. Hofmann, and W. W. Franke. Identification of a nonapeptide motif in the vimentin head domain involved in intermediate filament assembly. Journal of Molecular Biology, 223(3):637-650, 1992. (cited on page 10.

[49] D. A. Jacques and J. Trewhella. Small-angle scattering for structural biologyexpanding the frontier while avoiding the pitfalls. Protein Science, 19(4):642657, 2010. (cited on page 10.

[50] M. E. Brennich. Cation induced self-assembly of intermediate filaments. 2013. (cited on page 11 and 36

[51] M. E. Brennich and S. Köster. Tracking reactions in microflow. Microfluidics and Nanofluidics, 16(1-2):39-45, 2014. (cited on page 13 and 49

[52] C. Dammann, B. Nöding, and S. Köster. Vimentin networks at tunable ionconcentration in microfluidic drops. Biomicrofluidics, 6(2):22009-2200910, 2012. (cited on page $14,26,29,62,64,67,74$, and 84,

[53] C. Dammann and S. Köster. Dynamics of counterion-induced attraction between vimentin filaments followed in microfluidic drops. Lab on a Chip, 14(15):2681-2687, 2014. (cited on page 14, 62, 64 , 74 and 84

[54] C. Dammann, H. Herrmann, and S. Köster. Competitive counterion binding regulates the aggregation onset of vimentin intermediate filaments. Israel Journal of Chemistry, 2015. (cited on page 14,496264 and 84 . 
[55] D. I. Svergun and M. H. J. Koch. Small-angle scattering studies of biological macromolecules in solution. Reports on Progress in Physics, 66(10):1735, 2003. (cited on page 14, 39, 40, 49, and 101)

[56] H. Song, D. L. Chen, and R. F. Ismagilov. Reactions in droplets in microfluidic channels. Angewandte Chemie (International ed. in English), 45(44):7336-7356, 2006. (cited on page 15 .

[57] L. Li and R. F. Ismagilov. Protein crystallization using microfluidic technologies based on valves, droplets, and slipchip. Annual Review of Biophysics, 39:139-158, 2010. (cited on page 15

[58] J. Clausell-Tormos, D. Lieber, J. C. Baret, A. El-Harrak, O. J. Miller, L. Frenz, J. Blouwolff, K. J. Humphry, S. Köster, H. Duan, C. Holtze, D. A. Weitz, A. D. Griffiths, and C. A. Merten. Droplet-based microfluidic platforms for the encapsulation and screening of Mammalian cells and multicellular organisms. Chemistry \& Biology, 15(5):427-437, 2008. (cited on page 15)

[59] E. M. Purcell. Life at low reynolds number. American Journal of Physics, 45(1):3, 1977. (cited on page i 16, and 17 ,

[60] P. Tabeling. Introduction to Microfluidics. Oxford University Press, Oxford and New York, 2010. (cited on page 16 and 19 )

[61] R. Salvi. The Navier-Stokes equations: theory and numerical methods, volume v. 223 of Lecture Notes in Pure and Applied Mathematics. Marcel Dekker, New York, 2002. (cited on page16)

[62] Data sheet for su-8 3000 permanent epoxy negative photoresist. http:// microchem.com/pdf/SU-8\%203000\%20Data\%20Sheet.pdf, 2009. last checked: July 2014. (cited on page 19

[63] O. Saldanha, M.E. Brennich, M. Burghammer, H. Herrmann, and S. Köster. The filament forming reactions of vimentin tetramers studied in a serialinlet microflow device by small angle $x$-ray scattering. Biomicrofluidics, 10(2):024108, 2016. (cited on page 21, 26, 29, 31, 34, 35, 36, 37, 56, 57, 58, 59, 60, 61, 62. 636465 and 107

[64] H. E. H. Meijer, M. K. Singh, T. G. Kang, J. M. J. den Toonder, and P. D. Anderson. Passive and active mixing in microfluidic devices. Macromolecular Symposia, 279(1):201-209, 2009. (cited on page 25 
[65] H. Song and R. F. Ismagilov. Millisecond kinetics on a microfluidic chip using nanoliters of reagents. Journal of the American Chemical Society, 125(47):14613-14619, 2003. (cited on page26

[66] S. L. Anna, N. Bontoux, and H. A. Stone. Formation of dispersions using flow focusing in microchannels. Applied Physics Letters, 82(3):364, 2003. (cited on page 26)

[67] D. Bartolo, G. Degré, P. Nghe, and V. Studer. Microfluidic stickers. Lab on a Chip, 8(2):274-279, 2008. (cited on page 28 and 74 .

[68] Z. T. Cygan, J. T. Cabral, K. L. Beers, and E. J. Amis. Microfluidic platform for the generation of organic-phase microreactors. Langmuir, 21(8):3629-3634, 2005. (cited on page 28

[69] B. Zheng, J. D. Tice, L. S. Roach, and R. F. Ismagilov. A droplet-based, composite pdms/glass capillary microfluidic system for evaluating protein crystallization conditions by microbatch and vapor-diffusion methods with on-chip x-ray diffraction. Angewandte Chemie (International ed. in English), 43(19):2508-2511, 2004. (cited on page 29, 74, 78, and 103,

[70] K. H. Huebner and E. A. Thornton. The Finite Element Method for Engineers. John Wiley \& Sons, Inc., New York, second edition, 1982. (cited on page 30)

[71] S. A. Pabit and S. J. Hagen. Laminar-flow fluid mixer for fast fluorescence kinetics studies. Biophysical Journal, 83:2872-2878, 2002. (cited on page 32

[72] L. Pollack, M. W. Tate, A. C. Finnefrock, C. Kalidas, S. Trotter, N. C. Darnton, L. Lurio, R. H. Austin, C. A. Batt, S. M. Gruner, and S. G. J. Mochrie. Time-resolved collapse of a folding protein observed with small angle $x$-Ray scattering. Physical Review Letters, 86(21):4962-4965, 2001. (cited on page 32 and 77

[73] M. E. Kinahan, E. Filippidi, S. Köster, X. Hu, H. M. Evans, T. Pfohl, D. L. Kaplan, and J. Wong. Tunable silk: using microfluidics to fabricate silk fibers with controllable properties. Biomacromolecules, 12(5):1504-1511, 2011. (cited on page 32

[74] M. E. Young, P.A. Carroad, and R. L. Bell. Estimation of diffusion coefficients of proteins. Biotechnology and Bioengineering, 22:947-955, 1980. (cited on page 33. 
[75] H. S. Harned and R. L. Nuttall. The diffusion coefficient of potassium chloride in dilute aqueous solution. Journal of American Chemical Society, 69(4):736-740, 1947. (cited on page 33

[76] H. S. Harned and F. M. Polestra. The differential diffusion coefficients of magnesium and barium chlorides in dilute aqueous solutions at $25^{\circ}$. Journal of American Chemical Society, 76(8):2064-2065, 1954. (cited on page 33)

[77] N. Mücke, L. Kreplak, R. Kirmse, T. Wedig, H. Herrmann, U. Aebi, and J. Langowski. Assessing the flexibility of intermediate filaments by atomic force microscopy. Journal of Molecular Biology, 335(5):1241-1250, 2004. (cited on page 34

[78] U. Pietsch, V. Holý, and T. Baumbach. High-resolution X-ray Scattering: From Thin films to Lateral Nanostructures. Advanced Texts in Physics. Springer, New York, second edition, 2004. (cited on page 42 ,

[79] P. Debye and A. M. Bueche. Scattering by an inhomogeneous solid. Journal of Applied Physics, 20(6):518, 1949. (cited on page 43 )

[80] L. A. Feigin and D. I. Svergun. Structure Analysis by Small-angle X-ray and Neutron Scattering. Plenum Press, New York., first edition, 1987. (cited on page 44,60 and 101

[81] B. Jacrot. The study of biological structures by neutron scattering from solution. Reports on Progress in Physics, 39(10):911, 1976. (cited on page 44.

[82] A. Guinier and G. Fournet. Small-angle Scattering of X-rays. John Wiley \& Sons, Inc., New York., first edition, 1955. (cited on page 45)

[83] G. Porod. Die Röntgenkleinwinkelstreuung von dichtgepackten kolloiden Systemen. Colloid and Polymer Science, 124(2):83-114, 1951. (cited on page $45,46,58,51,59$ and 62

[84] Tutorial "What is measured in SAXS". http://iramis.cea.fr/Phocea/Vie_ des_labos/Ast/ast_sstechnique.php?id_ast=1065. last checked: February 2015. (cited on page 46 ,

[85] J. Als-Nielsen and D. McMorrow. Elements of Modern X-ray Physics. John Wiley \& Sons, Inc., New York., second edition, 2011. (cited on page 48 
[86] J. Teixeira. Small-angle scattering by fractal systems. Journal of Applied Crystallography, 21(6):781-785, 1988. (cited on page 49 and 62.

[87] G. P. Shrivastav, V. Banerjee, and S. Puri. Non-porod behavior in systems with rough morphologies. The European Physical Journal. E, Soft Matter, 37(10):98, 2014. (cited on page 49 and 62

[88] G. Beaucage. http://www.eng.uc.edu/ beaucag/BeaucageResearchGroup. html. last checked: February 2016. (cited on page 50

[89] A. Banerji. Fractal symmetry of protein exterior. SpringerBriefs in biochemistry and molecular biology. Springer, Basel and New York, 2013. (cited on page 50

[90] R. J. Davies, M. Burghammer, and C. Riekel. An overview of the ESRF's ID13 microfocus beamline. Synchrotron Radiation in Natural Science, 5(12):96-99, 2006. (cited on page 51

[91] H. Amenitsch, M. Rappolt, M. Kriechbaum, H. Mio, P. Laggner, and S. Bernstorff. First performance assessment of the small-angle X-ray scattering beamline at ELETTRA. Journal of Synchrotron Radiation, 5(3):506-508, 1998. (cited on page 53)

[92] R. Stehle, G. Goerigk, D. Wallacher, M. Ballauff, and S. Seiffert. Small-angle x-ray scattering in droplet-based microfluidics. Lab on a Chip, 13(8):15291537, 2013. (cited on page 546964 and 78

[93] O. Bunk, M. Bech, T. H. Jensen, R. Feidenhans'l, T. Binderup, A. Menzel, and F. Pfeiffer. Multimodal x-ray scatter imaging. New Journal of Physics, 11(12):123016, 2009. (cited on page 55)

[94] O. Glatter and O. Kratky. Small Angle X-ray Scattering. Academic Press, London, 1982. (cited on page 60 and 78

[95] P. Debye, H. R. Anderson, and H. Brumberger. Scattering by an inhomogeneous solid. ii. the correlation function and its application. Journal of Applied Physics, 28(6):679, 1957. (cited on page 62)

[96] H. Bale and P. W Schmidt. Small angle x-ray scattering investigation of submicroscopic porosity with fractal properties. Physical Review Letters, 53(6):596-599, 1984. (cited on page 62 
[97] B. Weinhausen and S. Köster. Microfluidic devices for x-ray studies on hydrated cells. Lab on a Chip, 13(2):212-215, 2013. (cited on page 74)

[98] S. Köster, H. M. Evans, J. Y. Wong, and T. Pfohl. An in situ study of collagen self-assembly processes. Biomacromolecules, 9(1):199-207, 2008. (cited on page77

[99] T. Pfohl, A. Otten, S. Köster, R. Dootz, B. Struth, and H. M. Evans. Highly packed and oriented dna mesophases identified using in situ microfluidic x-ray microdiffraction. Biomacromolecules, 8(7):2167-2172, 2007. (cited on page 77)

[100] A. Otten, S. Köster, B. Struth, A. Snigirev, and T. Pfohl. Microfluidics of soft matter investigated by small-angle x-ray scattering. Journal of Synchrotron Radiation, 12(Pt 6):745-750, 2005. (cited on page 78

[101] H. Schnablegger and Y. Singh. The saxs guide, $3^{\text {rd }}$ edition. http: //www.anton-paar.com/static/Blaetterkatalog/saxs-guide/, 2013. last checked: February 2016. (cited on page 78)

[102] H. N. Chapman. X-ray imaging beyond the limits. Nature Materials, 8(4):299-301, 2009. (cited on page79)

[103] H. N. Chapman, P. Fromme, A. Barty, T. A. White, R. A. Kirian, A. Aquila, M. S. Hunter, J. Schulz, D. P. DePonte, U. Weierstall, R. B. Doak, Maia, F. R. N. C, A. V. Martin, I. Schlichting, L. Lomb, R. L. Coppola, N.and Shoeman, S. W. Epp, R.t Hartmann, D. Rolles, A. Rudenko, L. Foucar, N. Kimmel, G. Weidenspointner, P. Holl, M. Liang, M. Barthelmess, C. Caleman, S. Boutet, M. J. Bogan, J. Krzywinski, C. Bostedt, S. Bajt, L. Gumprecht, B. Rudek, B. Erk, C. Schmidt, A. Hömke, C. Reich, D. Pietschner, L. Strüder, G. Hauser, H. Gorke, J. Ullrich, S. Herrmann, G. Schaller, F. Schopper, H. Soltau, K. U. Kühnel, M. Messerschmidt, J. D. Bozek, S. P. Hau-Riege, M. Frank, C. Y. Hampton, R. G. Sierra, D. Starodub, G. J. Williams, J. Hajdu, N. Timneanu, M. M. Seibert, J. Andreasson, A. Rocker, Olof J., M. Svenda, K.and Andritschke R. Stern, S.and Nass, C. D. Schröter, F. Krasniqi, M. Bott, K. E. Schmidt, X. Wang, I. Grotjohann, J. M. Holton, T. R. M. Barends, R. Neutze, S. Marchesini, R. Fromme, S. Schorb, D. Rupp, M. Adolph, T. Gorkhover, I. Andersson, H. Hirsemann, G. Potdevin, H. Graafsma, 
B. Nilsson, and J. C. H. Spence. Femtosecond x-ray protein nanocrystallography. Nature, 470(7332):73-77, 2011. (cited on page 79)

[104] R. P. Rambo. http://www.bioisis.net/tutorial/18, last checked: February 2016. (cited on page 80.

[105] S. Finet and A. Tardieu. $\alpha$-crystallin interaction forces studied by small angle x-ray scattering and numerical simulations. Journal of Crystal Growth, 232(1-4):40-49, 2001. (cited on page 80

[106] A. V. Sokolova, L. Kreplak, T. Wedig, N. Mücke, D. I. Svergun, H. Herrmann, U. Aebi, and S. V. Strelkov. Monitoring intermediate filament assembly by small-angle $x$-ray scattering reveals the molecular architecture of assembly intermediates. Proceedings of the National Academy of Sciences of the United States of America, 103(44):16206-16211, 2006. (cited on page 83)

[107] R. Michels, T. Sinemus, J. Hoffmann, B. Brutschy, and K. Huber. Coaggregation of two anionic azo dyestuffs at a well-defined stoichiometry. The Journal of Physical Chemistry. B, 117(28):8611-8619, 2013. (cited on page 84 and 92

[108] C. G. Lopez, O. Saldanha, K. Huber, and S. Köster. Lateral association and elongation of vimentin intermediate filament proteins: A time-resolved light-scattering study. Proceedings of the National Academy of Sciences, page 201606372, 2016. (cited on page 84 and 98 ,

[109] B. H. Zimm. Apparatus and methods for measurement and iinterpretation of the angular variation of light scattering; preliminary results on polystyrene solutions. The Journal of Chemical Physics, 16(12):1099, 1948. (cited on page 87)

[110] B.H. Zimm. The scattering of light and the radial distribution function of high polymer solutions. The Journal of Chemical Physics, 16(12):1093, 1948. (cited on page 87)

[111] J.S Higgins and H. Benoît. Polymers and neutron scattering. Clarendon Press Oxford, 1994. (cited on page 87)

[112] T. Neugebauer. Berechnung der Lichtzerstreuung von Fadenkettenlösungen. Annalen der Physik, 434(7-8):509-533, 1943. (cited on page 87) 
[113] M. Schmidt. Combined integrated and dynamic light scattering by poly ( $\gamma$ benzyl glutamate) in a helocogenic solvent. Macromolecules, 17(4):553-560, 1984. (cited on page 87

[114] H. Yamakawa. Modern theory of polymer solutions. Harper \& Row, 1971. (cited on page 88

[115] R. Pecora. Dynamic light scattering: applications of photon correlation spectroscopy. Springer Science \& Business Media, 2013. (cited on page 88 and 101

[116] D. G. Dalgleish and F. R. Hallett. Dynamic light scattering: Applications to food systems. Food Research International, 28(3):181-193, 1995. (cited on page 88

[117] M. Alexander and D. G. Dalgleish. Dynamic light scattering techniques and their applications in food science. Food Biophysics, 1(1):2-13, 2006. (cited on page 88

[118] B. J. Frisken. Revisiting the method of cumulants for the analysis of dynamic light-scattering data. Applied Optics, 40(24):4087, 2001. (cited on page 90 )

[119] A. G. Mailer, P. S. Clegg, and P. N. Pusey. Particle sizing by dynamic light scattering: non-linear cumulant analysis. Journal of Physics: Condensed Matter, 27(14):145102, 2015. (cited on page90

[120] P. A. Hassan and S. K. Kulshreshtha. Modification to the cumulant analysis of polydispersity in quasielastic light scattering data. Journal of Colloid and Interface Science, 300(2):744-748, 2006. (cited on page 90

[121] J. C. Selser. A determination of polymer number-averaged and weight-averaged molecular weight using photon correlation spectroscopy. Macromolecules, 12(5):909-916, 1979. (cited on page90)

[122] L. Harnau, R. G. Winkler, and P. Reineker. Influence of polydispersity on the dynamic structure factor of macromolecules in dilute solution. Macromolecules, 32(18):5956-5960, 1999. (cited on page90)

[123] H. Zhao, P. H. Brown, and P. Schuck. On the distribution of protein refractive index increments. Biophysical Journal, 100(9):2309-2317, 2011. (cited on page 93 
[124] J. Liu, J. Rieger, and K. Huber. Analysis of the nucleation and growth of amorphous caco3 by means of time-resolved static light scattering. Langmuir, 24(15):8262-8271, 2008. (cited on page 96 ,

[125] S. J. Tomski and R. M. Murphy. Kinetics of aggregation of synthetic $\beta$ amyloid peptide. Archives of Biochemistry and Biophysics, 294(2):630-638, 1992. (cited on page 97 and 127 .

[126] M. M. Pallitto and R. M. Murphy. A mathematical model of the kinetics of $\beta$ - amyloid fibril growth from the denatured state. Biophysical Journal, 81(3):1805-1822, 2001. (cited on page 97, 100, 127. and 129.

[127] T. L. Hill. Length dependence of rate constants for end-to-end association and dissociation of equilibrium linear aggregates. Biophysical Journal, 44(2):285-288, 1983. (cited on page 97 and 106

[128] S. Portet. Dynamics of in vitro intermediate filament length distributions. Journal of Theoretical Biology, 332:20-29, 2013. (cited on page 97

[129] M. M. Tirado and J. G. de la Torre. Rotational dynamics of rigid, symmetric top macromolecules. application to circular cylinders. The Journal of Chemical Physics, 73(4):1986, 1980. (cited on page 97)

[130] M. M. Tirado and J. G. de la Torre. Translational friction coefficients of rigid, symmetric top macromolecules. application to circular cylinders. The Journal of Chemical Physics, 71(6):2581, 1979. (cited on page 97)

[131] H. Herrmann and U. Aebi. Intermediate filament assembly: temperature sensitivity and polymorphism. Cellular and Molecular Life Sciences CMLS, 55(11):1416-1431, 1999. (cited on page 100

[132] R. C. Oberthür. Die Makromolekulare Chemie, 179(11):2693-2706, 1978. (cited on page 105 )

[133] M. U. Bäbler and M. Morbidelli. Analysis of the aggregation-fragmentation population balance equation with application to coagulation. Journal of Colloid and Interface Science, 316(2):428-441, 2007. (cited on page 106 


\section{List of Figures}

2.1 Cytoskeletal organization in metazoan cells . . . . . . . . . . 5

2.2 Fluorescence microscopy image of a cell . . . . . . . . . . . . 6

2.3 Sketch of the hierarchical structure of vimentin assembly. . . . . . . 9

2.4 Confocal fluorescence microscopy and AFM images of vimentin fil-

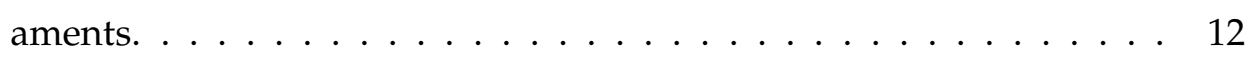

3.1 Sketch showing difference between convection and diffusion . . . . 18

3.2 Representative sketch of the photomasks . . . . . . . . . . . 20

3.3 Schematic diagram of device fabrication for continuous flow exper-

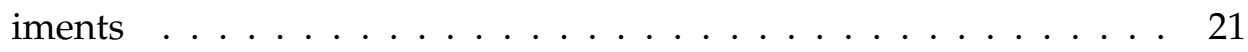

3.4 Photograph of the assembled droplet device for SAXS . . . . . . . . 24

3.5 Photograph of the assembled droplet device for microscopy . . . . . 24

3.6 Simulated hydrodynamic flow focusing in continuous flow device . 26

3.7 Schematic diagram of the "T-junction" geometry for droplet exper-

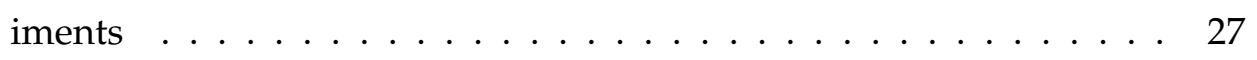

3.8 Sketch of the continuous flow device geometry . . . . . . . . . . . . 29

3.9 Sketch of the droplet device . . . . . . . . . . . . . . . 30

3.10 Comparison of attenuation lengths . . . . . . . . . . . . . . . 31

3.11 Geometry of the channel used for FEM simulations of continuous

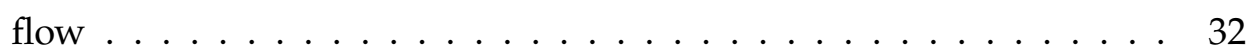

3.12 FEM simulations of the flow conditions and local concentrations in the microfluidic devices . . . . . . . . . . . . . . . 35

3.13 Results derived from the FEM simulations for continuous flow . . . 36

3.14 Time evolution along the length of the channel for simulations of different flow rates . . . . . . . . . . . . . . 37

4.1 Schematic X-ray scattering diagram . . . . . . . . . . . . . . . 41

4.2 Simple representative sketch to understand the resolution limits . . 46

4.3 Sketch of the correlation between exponents and fractals. . . . . . . . 50 
4.4 Demonstration of geometric self-similarity. . . . . . . . . . . . . . 50

4.5 Schematic diagram of the beamline components at ID13 experimental hutch II, ESRF. . . . . . . . . . . . . . . . . . . . . . 52

4.6 Schematic diagram of the beamline components at AustroSAXS,

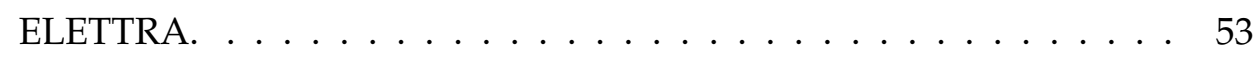

4.7 Schematic diagram of the beamline components at cSAXS, SLS. . . . 54

$4.8 \quad$ X-ray measurement strategy for continuous flow experiments . . . . 58

4.9 Experimental results of SAXS for continuous flow . . . . . . . . . 59

4.10 Fit determining the correct $q_{r}$ range for Porod's analysis for X-ray

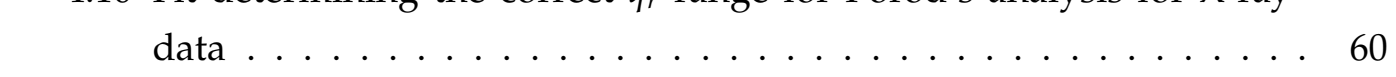

4.11 Fit exponents from Porod's analysis for continuous flow experiments 61

4.12 Results derived from the FEM simulations of continuous flow . . . . 61

4.13 Fit exponents as a function of $\mathrm{KCl}$ concentration for continuous flow

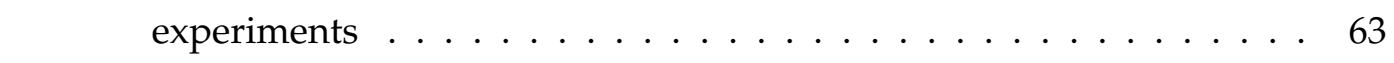

4.14 Droplet-mapping with SAXS . . . . . . . . . . . . . 67

4.15 High resolution measurement technique for droplet-mapping . . . . 68

4.16 Azimuthally integrated 1D-scattering intensity profiles from data collected at ESRF . . . . . . . . . . . . . . . . 69

4.17 Integrated 1D-scattering intensity profiles for vimentin aggregation 69

4.18 Data collected for vimentin assembly in droplets at ELETTRA . . . 70

4.19 Control experiments for vimentin signal characterization . . . . . . . 72

4.20 Data collected for vimentin assembly in droplets from cSAXS, SLS . 73

4.21 Comparison of vimentin assembly between flow focusing and droplets 75

4.22 Comparison of unassembled vimentin data . . . . . . . . . . 76

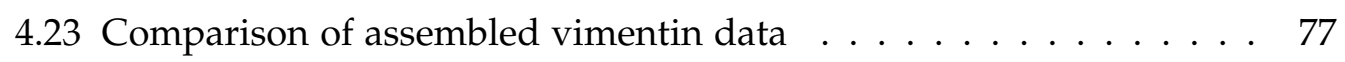

4.24 Demonstration of scattered intensity variation as a function of protein concentration. .............. 80

5.1 Schematic Static Light Scattering Image . . . . . . . . . . . . . . . 88

5.2 Photograph of the 8-angled light scattering instrument . . . . . . . 92

5.3 Photograph of the single-angled detection light scattering instrument 92

$5.4 \quad$ Representative results of the influence of $\mathrm{KCl}$ on vimentin . . . . . . 94

$5.5 \quad R_{g}$ vs. $M_{w}$ for the kinetic run from Fig. $5.4 \ldots \ldots \ldots$

$5.6 R_{H}$ vs. $L_{w}$ with model fit . . . . . . . . . . . . . . . . . 96

$5.7 \quad M_{w}$ vs. time and model fit . . . . . . . . . . . . . . . . 97

5.8 Scheme for the assembly of vimentin $\ldots \ldots \ldots$. . . . . . . . 98 
$5.9 \quad M_{w}$ vs. time for 3 different kinetic runs $\ldots \ldots \ldots$. . . . . . . . . 99

6.1 Overview scheme of the length and time scales accessible with out

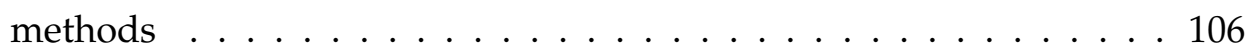

C.1 FEM simulations of the flow conditions and local concentrations in the microfluidic devices for flow rates 40:20 . . . . . . . . . . . . . . 124

C.2 FEM simulations of the flow conditions and local concentrations in the microfluidic devices for flow rates 160:80 . . . . . . . . . . . . . 125 


\section{List of Tables}

2.1 Classification of the intermediate filament proteins into six categories. 7

3.1 Diffusion coefficients of different species used in the FEM simulations 33

$4.1 \quad$ Approximate time points corresponding to various stages of vimentin assembly based on droplet flowrate. . . . . . . . . . . 71

5.1 Accessible real-space length scales for visible light and X-rays. . . . 85

$5.2 \quad M_{w}, R_{g}, R_{H}$ and $M_{L}$ for samples used for kinetic runs $\ldots . . . .100$ 


\section{List of Abbreviations}

IF

SAXS

LS

SLS

DLS

DNA

RNA

MF

MT

INM

EMT

ECM

ULF

TIRFM

$\mathrm{UV} / \mathrm{Vis}$

A

$\epsilon$

$l_{p}$

$c$

ATP

GTP

$\mathrm{KCl}$

$\mathrm{MgCl}_{2}$

$\mathrm{K}^{+}$

$\mathrm{Mg}^{2+}$
Intermediate filament

Small angle $\mathrm{X}$-ray scattering

Light scattering

Static light scattering

Dynamic light scattering

Deoxyribonucleic acid

Ribonucleic acid

Microfilaments, actin filaments

Microtubules

Inner nuclear membrane

Epithelial-mesenchymal transition

Extra cellular matrix

Unit-length-flament (an intermediate of IF formation)

Total internal reflection florescence microscopy

Ultraviolet/Visible

Absorbance of a sample

Extinction coefficient of a sample

Optical path

Protein concentration

Adenosintriphosphate

Guanosintriphosphate

Potassium chloride

Magnesium chloride

Potassium-monovalent ion

Magnesium-divalent ion 


$\begin{array}{ll}\text { mM } & \text { Millimolar - unit of molar concentration }\left(10^{-3} \mathrm{~mol} / \mathrm{L}\right) \\ \text { AFM } & \text { Atomic force microscopy } \\ \text { EM } & \text { Electron microscopy } \\ \text { TEB } & \text { Transient electric birefringence } \\ \text { PDMS } & \text { Polydimethylsiloxane } \\ r h o & \text { Electron density } \\ V & \text { Sample volume } \\ I_{0} / I_{i} & \text { Incident intensity } \\ q, \vec{q} & \text { Scattering vector } \\ \mu \mathrm{m} & \text { Micrometer - unit of length }\left(10^{-6} \mathrm{~m}\right) \\ \mathrm{FEM} & \text { Finite element method } \\ e t a & \text { Dynamic viscosity } \\ \vec{v} & \text { Velocity field } \\ \vec{f} & \text { Applied external force per volume } \\ \operatorname{Re} & \text { Reynolds number } \\ P & \text { Pressure } \\ U & \text { Initial velocity } \\ P e & \text { Péclet number } \\ D & \text { Diffusion coefficient } \\ \mathrm{NOA}-81 & \text { Norland optical adhesive } \\ \mathrm{HMDS} & \text { Hexamethyldisilazane } \\ \mathrm{mm} & \text { Millimeter-unit of length }\left(10^{-3} \mathrm{~m}\right) \\ \gamma & \text { Shear rate } \\ \tau & \text { Shear stress } \\ \Delta x, \Delta y & \text { Step size in } x, y \text { direction } \\ \vec{k} & \text { Reciprocal vector of incoming wave } \\ \vec{k}{ }^{\prime} & \text { Reciprocal vector of scattered wave } \\ n & \text { Number of electrons } \\ \vec{r} & \text { Spatial variable } \\ \rho(\vec{r}) & \text { Charge density distribution } \\ \vec{E} & \text { Electric field strength } \\ A(\vec{q}), A(\vec{q})^{*} & \text { Scattering amplitude and its complex conjugate } \\ P(\vec{q}) & \text { Form factor of a particle } \\ S(\vec{q}) & \text { Structure factor of the particle } \\ \mathrm{ESRF} & \text { European synchrotron radiation facility } \\ \mathrm{PSI} & \text { Paul Scherrer institute } \\ \mathrm{Si} & \text { Silicon } \\ & \end{array}$




$\begin{array}{ll}\mathrm{KB} & \text { Kirkpatrick- Baez (mirrors used for focusing) } \\ \mathrm{keV} & \text { Kilo electronvolt - unit of energy } \\ \mathrm{CCD} & \text { Charge-coupled device } \\ p(r) & \text { Particle distance distribution function } \\ R_{g} & \text { Radius of gyration } \\ d_{f} & \text { Power law exponent } \\ h \times v & \text { Horizontal x vertical } \\ l & \text { Length of a microfluidic channel } \\ M_{w} & \text { Weight-averaged molecular weight of the solute } \\ R_{\theta} & \text { Excess Rayleigh ratio } \\ N_{A} & \text { Avagadro's number } \\ n_{s} & \text { Refractive index of the solvent } \\ \lambda & \text { Wavelength of a incident radiation } \\ L_{w} & \text { Weight-average of particle length } \\ A_{2} & \text { Virial cofficients for particle shapes } \\ g & \text { Auto-correlation function } \\ T & \text { Temperature } \\ R_{H} & \text { Hydrodynamic radius of the particle } \\ G(\Gamma) & \text { Distribution of decay times } \\ \mu_{n} & \text { Cumulant moments } \\ \mathrm{HeNe} & \text { Helium-Neon laser } \\ M_{l} & \text { Mass per unit length } \\ c_{p} & \text { Protein sample concentration } \\ c_{s} & \text { Salt sample concentration }\end{array}$





\section{Acknowledgements}

No man/woman is an island and I have indeed had the privilege to be around the finest people and facilities in order to be able to work on this thesis. It is now time for me to extend my heartfelt gratitude to them. My thanks goes to:

Göttingen, for the town that it is. Only destiny has pulled me here.

My supervisor, Prof. Dr. Sarah Köster, who has a bottomless well of patience, who gave me the opportunity to work on this subject and for the excellent support all this while. I owe it all to her.

Dr. Jochen Hub, for obliging to be the second referee of this thesis and a part of the examination committee.

Prof. Dr. Simone Techert and Prof. Dr. Jean-Christophe Baret, for being members of my thesis committee and a part of the examination board.

Dr. Thomas Burg and Prof. Dr. Detlev Schild, for agreeing to be a part of the examination committee.

The Helmholtz Gemeinschaft in the framework of Virtual Institute VH-VI403 In-Situ Nano-Imaging of Biological and Chemical Processes, for funding the research work performed in this thesis.

GGNB and IMPRS Physics of Biological and Complex Systems, for providing a good structure at the graduate school. 
Dr. Manfred Burghammer and Dr. Britta Weinhausen, for assistance during beamtimes. For accommodating our needs and necessities and for giving us the chance to perform our experiments at ID13, ESRF.

Dr. Benedetta Marmiroli, Dr. Barbara Sartori and Prof. Dr. Heinz Amenitsch for the assistance during the beamtime at ELETTRA.

Members of the cSAXS beamline in Switzerland, for providing us very good assistance during beamtimes.

Prof. Dr. Harald Herrmann, for his insightful ideas with regard to vimentin.

Prof. Dr. Tim Salditt and the secretaries Sabine and Kerstin, for assistance be it official paperwork or otherwise.

Members of the IRP, for the lively atmosphere at the institute, for technical assistance and knock-on-the-door questions on various occasions, for the cakes, Christmas parties and sports.

Susanne Bauch, for the technical assistance with the vimentin and buffer preparations for all the umpteen experiments.

Dr. Martha Brennich, for her useful suggestions and for preparing a good groundwork in the subject.

Dr. Christian Dammann, for being a helpful colleague in the past and for his ideas, tips and tricks with the microfluidics.

Prof. Dr. Klaus Huber, for the collaboration for light scattering experiments at the University of Paderborn and for giving me the opportunity to perform experiments in his group. Dr. Carlos Lopez, for he has been a very patient teacher and a pleasant colleague throughout. Dr. Sanjib Saha, for his timely ideas and help during the measurements.

Sabin Prajapati and Christian Rossner, for their interest on measuring at our in-house Kratky set-up which has culminated in fruitful discussions with respect to X-ray data. 
Jochen Herbst, for clean-room assistance and Peter Nieschalk, for assistance in the machine shop.

A loud shout out to all my colleagues from the AG Koester.

Tim, Heidi, Johanna, Jana, Jens, Bernd, Chiara, Gerrit, Manuela, Eleonora, Daniel and all the past members for providing a wonderful working environment.

Aishwarya, Rabea, Christiane and Viktor, for being the wonderful colleagues that you are! From timely help at work to food, music and dances, I've cherished it all.

Rita and Clément, for their time and energy during the X-ray experiments, for their critical yet fruitful discussions on data analysis and nuances of $\mathrm{X}$-ray science.

My small Indian fraternity in Göttingen "Nirmiti" and the members who have become dear friends Phani, Lenin, Kanagu, Navaneethan and Sankari for all the food, laughs, games, trials, tribulations and experiences we've had together as a motley crew. Sromona, Vinodh, Rita, Arun and Rose Mary, for your love and helpful conversations about everything under the sun. We are only not related by blood!. Priyanka and Samaneh, my room-mates who have been considerate and kind throughout.

Pawan, for his unique outlook on life in general and science in specific. Our story continues from Mysore to Göttingen and someplace in space (hopefully with Master Yoda) in the near future.

Albert "Abut" uncle, Bai, Fr. Edward "Thamma" uncle, Sr. Linda "Rita" aunty and my whole big fat Indian family for their love and blessings.

Karthikeyan, for he has been a pillar of constant relief, guidance, motivation and basically a good friend through the journey of my PhD. I thank you for being there for me, be it scientific or otherwise.

Lastly, it goes without saying, that my deepest and utmost gratitude is for my parents Louis and Joyce and my sister Pearl for their trust in me, for their undying support and for making me the person that I am. 



\section{List of Publications}

- Oliva Saldanha, Rita Graceffa, Clément Y.J. Hémonnot, Christiane Ranke, Gerrit Brehm, Marianne Liebi, Benedetta Marmiroli, Britta Weinhausen, Manfred Burghammer and Sarah Köster.

Rapid acquisition of X-ray scattering data from droplet-encapsulated protein systems

ChemPhysChem (2017), DOI: 10.1002/cphc.201700221.

- Clément Y.J. Hémonnot, Christiane Ranke, Oliva Saldanha, Rita Graceffa, Johannes Hagemann and Sarah Köster.

Following DNA Compaction During the Cell Cycle by X-Ray Nano-Diffraction ACS Nano 10 (2016), 10661-10670.

- Carlos G. Lopez*, Oliva Saldanha*, Klaus Huber and Sarah Köster.

* equal first authors

Lateral association and elongation of vimentin intermediate filament proteins: A time-resolved light-scattering study

Proceedings of the National Academy of Sciences 113 (2016), 11152-11157.

- Oliva Saldanha, Martha Brennich, Manfred Burghammer, Harald Herrmann and Sarah Köster.

The filament forming reaction of vimentin tetramers studied in a serial-inlet microflow device by small angle x-ray scattering

Biomicrofluidics 10 (2016), 024108.

- Clément Y.J. Hémonnot, Juliane Reinhardt, Oliva Saldanha, Jens Patommel, Rita Graceffa, Britta Weinhausen, Manfred Burghammer, Christian G. Schroer and Sarah Köster.

X-Rays Reveal the Internal Structure of Keratin Bundles in Whole Cells ACS Nano 10 (2016), 3553-3561. 
- Christian Rossner, Otto Glatter, Oliva Saldanha, Sarah Köster and Philipp Vana.

The Structure of Gold-Nanoparticle Networks cross-linked by Di- and Multifunctional RAFT Oligomers

Langmuir 31 (2015), 10573-10582.

- Britta Weinhausen, Oliva Saldanha, Robin N. Wilke, Christian Dammann, Marius Priebe, Manfred Burghammer, Michael Sprung and Sarah Köster.

Scanning X-ray Nano-Diffraction on Living Eukaryotic Cells in Microfluidic Environments

Physical Review Letters 112 (2014), 088102.

- Dileep Kumar, K.V. Sarathlal, Oliva Saldanha and Ajay Gupta. In-Situ Magneto-Optical Kerr Effect for Interface Magnetism Proceedings of the 55 $5^{\text {th }}$ DAE Solid State Physics Symposium 2010. AIP Conference Proceedings 1349 (2011), 1187-1188. 


\section{Curriculum Vitae}

Oliva Saldanha

\section{Personal Details}

Date of Birth

$28^{\text {th }}$ February 1989

Place of Birth in Virajpet, India

Nationality

Indian

\section{Education and Scientific Background}

2012 - present

2011

$2006-2011$

2006

2004
Graduate School, (Göttingen Graduate School for Neurosciences, Biophysics, and Molecular Biosciences (GGNB), Programm: International Max Planck Research School (IMPRS) Physics of Biological and Complex Systems).

Institute For X-ray Physics, Georg-August Universität Göttingen

Master of Sciences (Physics), Thesis titled "In-situ study of magnetic thin films: effect of substrate roughness and capping layer" in UGC- DAE Consortium for Scientific Studies, Indore, India

Five Year Integrated Master's in Physics, University of Mysore, India

Pre-University College, (12th grade equivalent), Teresian College, Mysore, India

High School 10th Grade, St Anne's Girls High School, Mysore, India 